\title{
TU/e EmonONEN

\section{Coupled aerostructural shape and topology optimization of horizontal-axis wind turbine rotor blades}

\section{Citation for published version (APA):}

Wang, Z. (2020). Coupled aerostructural shape and topology optimization of horizontal-axis wind turbine rotor blades. [Phd Thesis 1 (Research TU/e / Graduation TU/e), Built Environment]. Technische Universiteit Eindhoven.

Document status and date:

Published: 10/11/2020

\section{Document Version:}

Publisher's PDF, also known as Version of Record (includes final page, issue and volume numbers)

\section{Please check the document version of this publication:}

- A submitted manuscript is the version of the article upon submission and before peer-review. There can be important differences between the submitted version and the official published version of record. People interested in the research are advised to contact the author for the final version of the publication, or visit the $\mathrm{DOI}$ to the publisher's website.

- The final author version and the galley proof are versions of the publication after peer review.

- The final published version features the final layout of the paper including the volume, issue and page numbers.

Link to publication

\section{General rights}

Copyright and moral rights for the publications made accessible in the public portal are retained by the authors and/or other copyright owners and it is a condition of accessing publications that users recognise and abide by the legal requirements associated with these rights.

- Users may download and print one copy of any publication from the public portal for the purpose of private study or research.

- You may not further distribute the material or use it for any profit-making activity or commercial gain

- You may freely distribute the URL identifying the publication in the public portal.

If the publication is distributed under the terms of Article 25fa of the Dutch Copyright Act, indicated by the "Taverne" license above, please follow below link for the End User Agreement:

www.tue.nl/taverne

Take down policy

If you believe that this document breaches copyright please contact us at:

openaccess@tue.nl

providing details and we will investigate your claim. 
Coupled Aerostructural Shape and Topology Optimization of Horizontal-Axis Wind Turbine Rotor Blades

\title{
PROEFSCHRIFT
}

\author{
ter verkrijging van de graad van doctor \\ aan de Technische Universiteit Eindhoven, \\ op gezag van de rector magnificus prof.dr.ir. F.P.T. Baaijens, \\ voor een commissie aangewezen door het College voor Promoties, \\ in het openbaar te verdedigen op \\ dinsdag 10 november 2020 om 16:00 uur
}

door

Zhijun Wang

geboren te Sichuan, China 
Dit proefschrift is goedgekeurd door de promotoren en de samenstelling van de promotiecommissie is als volgt:

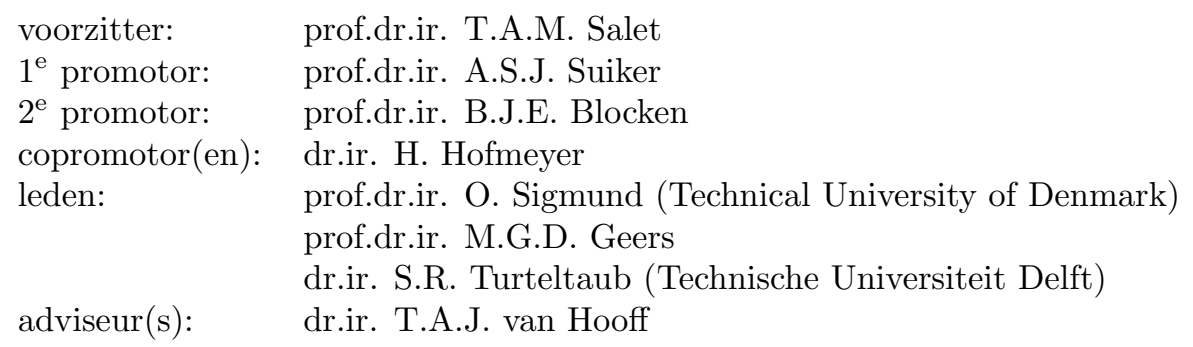

Het onderzoek of ontwerp dat in dit proefschrift wordt beschreven is uitgevoerd in overeenstemming met de TU/e Gedragscode Wetenschapsbeoefening. 
Coupled Aerostructural Shape and Topology Optimization of Horizontal-Axis Wind Turbine Rotor Blades

Zhijun Wang

October 11, 2020 

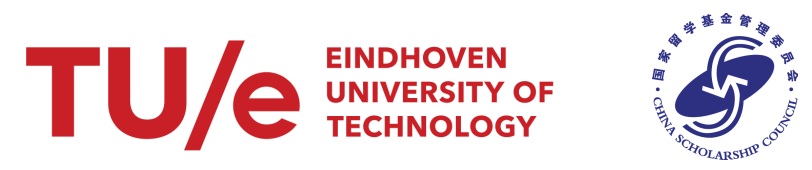

The research presented in this thesis was conducted at the Eindhoven University of Technology (TU/e) and was funded by the China Scholarship Council (CSC).

A catalogue record is available from the Eindhoven University of Technology Library. ISBN: 978-90-386-5135-4

Bouwstenen 303

NUR: 978

Cover design: Zhijun Wang

Printed by TU/e print service - Dereumaux, Eindhoven, The Netherlands

Copyright (C)Zhijun Wang, 2020

All rights reserved. No part of this publication may be reproduced, stored in a retrieval system, or transmitted, in any form or by any means, electronic, mechanical, photocopying, recording or otherwise, without the prior written permission from the copyright owner. 
"The art of structure is where to put the holes"

Robert Le Ricolais, 1894-1977 



\section{Acknowledgements}

The research work presented in this dissertation has been carried out from September 2015 to January 2020 in the Applied Mechanics (AM) and the Building Physics (BP) groups at the Eindhoven University of Technology (TU/e). Over the past four and half years, a number of people have been involved directly or indirectly with this work. I think this dissertation would never have been realized without their help and contributions. Therefore, I would like to take this opportunity to sincerely thank everyone who has guided, supported or accompanied me to get to the end.

My deep gratitude first goes to the supervisors, Prof.Dr. Akke Suiker, Prof.Dr. Bert Blocken, Dr. Hèrm Hofmeyer and Dr. Twan van Hooff, for their professional guidance, unwavering support and complete trust during the course of my Ph.D. study. Apart from sharing their expertise and research experience, they have also taught me how to do research and how to write a scientific paper. In particular, I would like to thank my first promoter Prof. Akke Suiker for accepting me to be part of his research group. I really enjoyed the pleasant working environment he creates, in which I was given plenty of freedom to shape the Ph.D. project, so that I could become an independent researcher. Further, he was always available for questions and discussions. Additionally, I am also very thankful to my previous co-supervisor Dr. Ivo Kalkman for offering the advice and help during the early stage of my Ph.D. work.

I would like to extend my thanks to the other members of the defense committee, Prof.Dr. Ole Sigmund, Prof.Dr. Marc Geers and Dr. Sergio Turteltaub, for spending time reviewing my thesis and giving valuable feedback. Further, the committee chair Prof.Dr. Theo Salet is also thanked for his work and time during the public defense.

The numerical implementation and academic exercise of this research partially benefit from the open source packages. Hereby I would like to thank to DTU (Technical University of Denmark) Wind Energy in Denmark and the NREL (National Renewable Energy Laboratory) in the U.S. for providing the open source software BECAS and CCBlade, respectively. I am also grateful to Prof. Krister Svanberg at KTH (Royal Institute of Technology) in Stockholm for providing the optimization solvers MMA and GCMMA. Moreover, Prof. Stéphane Bordas at the University of Luxembourg and his former Ph.D. student, Haojie Lian, are thanked for sharing their in-house code IGABEM. Further, I would like to extend my acknowledgement to NWO Exacte 
Wetenschappen (Physical Sciences) for providing supercomputer facilities. Regarding to this aspect, Dr. Rahim Rezaeiha and Dr. Hamid Montazeri at the BP group of the TU/e are highly appreciated for the help on applying for computational resource.

The financial support provided by the China Scholarship Council (CSC) is gratefully acknowledged. Relating to the CSC funding application, I would like to express my sincere gratitude to Prof. Tengjiao Lin, Prof. Wen Liu and Prof. Yun Huang at Chongqing University in China, as well as my friends Dr. Zeyin He, Yan Xie and Guangming Lan, for providing their support and help.

During my stay at TU/e, I have met a number of super kind and brilliant people. Hereby all the colleagues and friends of the 6,8 and $9^{\text {th }}$ floors of the Vertigo building are truly acknowledged for creating the enjoyable atmosphere, the nice chats and discussions, as well as many other interesting activities. Special thanks goes to my good friends Feiyu Geng and Sjonnie Boonstra; they play a role as my Chinese and Dutch brothers in Eindhoven. The floor 9 lunch group and ninja's sports team are also particularly thanked for bringing me so much fun during our working days. Furthermore, I enjoyed the time after work with my friends (both in and outside of my working place) for parties, trips and sports, and I would never ever forget our wonderful memories and genuine friendships.

After finishing the Ph.D. research work at the TU/e, I am lucky enough to get a postdoctoral position at the Faculty of Aerospace Engineering of the Delft University of Technology (TU Delft). Accordingly, I would like to take this opportunity to thank Dr. Roeland De Breuker, Dr. Daniël Peeters and Prof. Clemens Dransfeld at the TU Delft for offering me the postdoctoral position.

Last but not the least, I would like to thank my parents, my brother Zhiming Wang and his wife Huan Jiang, and my girlfriend Shawnee Simons and her parents. Zhiming and Huan, thank you very much for taking care of everything in China when I am abroad. Shawnee, you are always there for me, many thanks for your support and love, and I also enjoyed the time together with your family.

爸爸妈妈, 感谢您们一如既往的支持和无私奉献的爱, 我爱您们!

Zhijun Wang (王治军)

Den Haag, June 2020 


\section{Summary}

A desirable design of a wind turbine rotor generally requires higher power, less material and higher stiffness. Traditionally, the Aerodynamic Shape Optimization and Structural Topology Optimization (ASO-STO) applied for the aerostructural design of rotor blades are separated in two consecutive stages, which do not adequately account for the coupling effects between the blade aerodynamics and its structural performance. This limitation may be overcome by making use of a multi-objective optimization method. However, to the best of authors' knowledge, the existing multi-objective optimization methods for wind turbine blades are limited to prescribed internal topological layouts. In order to improve on this, this research concentrates on developing a Coupled Multi-objective Shape and Topology Optimization (CMSTO) framework, which aims to simultaneously optimize the blade outer shape and interior layout from aerodynamic and structural requirements.

Firstly, a coupled structural shape and topology optimization formulation is presented that is based on incorporating the shape design variables of the design domain in the SIMP (Solid Isotropic Material with Penalization) topology optimization method. To guarantee a highly accurate description and enable an efficient control of the boundary shape, Non-Uniform Rational B-Splines (NURBS) are employed to parameterize the outer shape of the structure. Furthermore, the shape and topology sensitivities are derived in closed form within a Finite Element Method (FEM) framework, so that gradient-based optimization algorithms with a high computational efficiency can be used. The coupled optimization approach has been implemented numerically for $2 \mathrm{D}$ configurations using a staggered numerical update scheme, and its usefulness has been demonstrated by analyzing several benchmark problems.

Secondly, the coupled optimization method presented is tailored towards the analyses of $2.5 \mathrm{D}$ and $3 \mathrm{D}$ beam models, which are representative for rotor blades used in Horizontal-Axis Wind Turbines (HAWTs). In the 2.5D beam model computationally efficient beam elements are used to simulate the beam response in the longitudinal direction, whereby the cross-sectional properties of the beam elements are calculated from additional 2D FEM analyses. In order to validate the accuracy and efficiency of the 2.5D optimization framework, a comparison study of a cantilever beam problem subjected to pure shape optimization and pure topology optimization has been conducted. The results illustrate that the $2.5 \mathrm{D}$ and $3 \mathrm{D}$ beam models lead to similar 
shape and topology designs, but that the $2.5 \mathrm{D}$ beam model has a significantly higher computational efficiency.

Thirdly, a CMSTO model is built based on the 2.5D beam formulation and the weighted sum method, which is used for the aerostructural optimization of HAWT rotor blades. Here, the rotor power coefficient and blade structural compliance are considered to be the two design objectives. To limit the computational demand and directly make use of the blade geometry information of $2.5 \mathrm{D}$ model, the Blade Element Momentum (BEM) method is adopted in the aerodynamic analysis of the rotor blade. The CMSTO framework is used to optimize the NREL 5MW reference rotor blade, whereby the results are compared to those obtained from a separate ASO-STO approach and a pure STO approach. The rotor power coefficients calculated by the CMSTO and ASO-STO approaches are about $4 \%$ larger than that of the reference rotor blade. In addition, the blade structural compliance computed by the CMSTO approach is reduced by an extra $16 \%$ and $41 \%$ compared to the compliances found by ASO-STO and pure STO approaches, respectively. Such significant improvements clearly demonstrate the benefits of the CMSTO approach in the quest of wind turbines with higher power output and better structural performance.

Finally, in addition to an implementation based on a staggered update scheme, the coupled optimization method has also been implemented based on a monolithic numerical update scheme. For investigating the effect of the solution strategy on the coupled optimization results, the performance of the monolithic and staggered numerical update schemes are compared in a systematic fashion, in which the $2.5 \mathrm{D}$ coupled optimization framework described above is applied for the design of thin-walled beam structures. The numerical examples concern basic circular and square cantilever beam structures, as well as a more complex, non-prismatic beam structure representative of a rotor blade used in a HAWT. For each numerical example the computational time and the solution computed by the monolithic and staggered update schemes are compared, which provides clear insight into the numerical efficiency of the solution procedure and the uniqueness of the computational result. In particular, the computational efficiency and the specific objective value found turn out to be rather sensitive to the algorithmic details of the update scheme applied. Despite this sensitivity, the three different update schemes for the cases examined result in comparable optimized design concepts, which provides confidence in the results calculated by the coupled optimization method. 


\section{Contents}

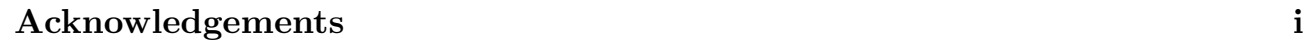

Summary iii

\begin{tabular}{lll}
\hline & Introduction & 1
\end{tabular}

1.1 Problem statement . . . . . . . . . . . . . . . . . . . . . . . 1

1.2 Research objectives and methodology . . . . . . . . . . . . . . . . 5

1.3 Thesis outline . . . . . . . . . . . . . . . . . . 6

2 Coupled shape and topology optimization method 9

2.1 Introduction . . . . . . . . . . . . . . . . . . . 10

2.2 Problem formulation and solution strategy . . . . . . . . . . . . . . . . 12

2.3 Geometry description . . . . . . . . . . . . . . . . . . . . . . . . . . . . . . . .

$2.3 .1 \quad$ B-spline surface . . . . . . . . . . . . . . . . . . . . . . . . . . . . . . . . . . . . . . . . 15

2.3 .2 NURBS surface . . . . . . . . . . . . . . . . . . . . . . . . . . . . . . . . . .

2.4 Structural analysis $\ldots \ldots \ldots \ldots \ldots \ldots$. . . . . . . . . . . . . . . . 17

2.5 Sensitivity analysis and update of design variables . . . . . . . . . . 21

2.5 .1 Shape sensitivity analysis . . . . . . . . . . . . . 21

2.5 .2 Topology sensitivity analysis . . . . . . . . . . . . . . . . . . . . 24

2.5 .3 Update of design variables . . . . . . . . . . . . . . . . . . . . . . . . . . . . . . . . . . 25

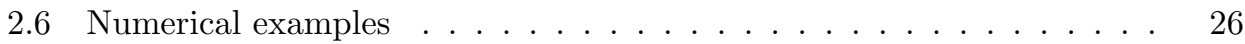

2.6.1 Cantilever beam problem . . . . . . . . . . . . . . 26

2.6 .2 Curved L-shape beam problem . . . . . . . . . . . . . . . 37

2.7 Concluding remarks $\ldots \ldots \ldots \ldots$. . . . . . . . . . . . . 40

\begin{tabular}{|lll}
\hline 3 & Coupled optimization of beam-type structures & 43
\end{tabular}

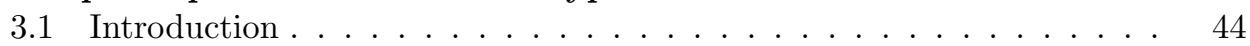

3.2 Geometry design model . . . . . . . . . . . . . . . . . . . . . . . . . . . . . . . . 45

$3.2 .1 \quad$ NURBS curve . . . . . . . . . . . . . . . . . . . . . . 46

3.2 .2 NURBS surface . . . . . . . . . . . . . . . . . . . . . . . . . . . . . . . . . 47

3.2 .3 NURBS solid . . . . . . . . . . . . . . . . . . . . . . . . . . . . . . . . . . . . . . . 48

3.3 Structural analysis model . . . . . . . . . . . . . . . . . . 48 
3.3.1 3D beam model . . . . . . . . . . . . . . . . . . . 49

3.3 .2 2.5D beam model . . . . . . . . . . . . . . . . . . . . . . . . . . . . . . . . 52

3.4 Coupled optimization model . . . . . . . . . . . . . . . . . . . . . 58

3.5 Sensitivity analysis $\ldots \ldots \ldots \ldots \ldots$. . . . . . . . . . . . . 60

3.5.1 2.5D beam model . . . . . . . . . . . . . . . . . . . . 60

3.5 .2 3D beam model . . . . . . . . . . . . . . . . . . . . 64

3.6 Numerical examples . . . . . . . . . . . . . . . . . . . . . . 66

3.6 .1 Validation case studies . . . . . . . . . . . . . . . 66

3.6.2 Coupled shape and topology optimization case studies . . . . . 73

3.7 Conclusions . . . . . . . . . . . . . . . . . . . . . 78

\begin{tabular}{|lll}
\hline Aerostructural design of turbine blades & 81
\end{tabular}

4.1 Introduction . . . . . . . . . . . . . . . . . 82

4.2 Coupled aerostructural optimization model . . . . . . . . . . . . . . 84

4.3 Numerical solution strategy . . . . . . . . . . . . . . . . . . . . . . . . . . . . . . .

4.3 .1 Geometry description . . . . . . . . . . . . . . . . . . 87

4.3 .2 Aerostructural analysis . . . . . . . . . . . . . . . . . 90

$4.3 .3 \quad$ Sensitivity analysis . . . . . . . . . . . . . . . . . . . 95

4.4 Simulation results $\ldots \ldots \ldots \ldots$. . . . . . . . . . . . . . . . . . . . 98

4.5 Conclusions $\ldots \ldots \ldots \ldots$

5 Comparison of numerical update schemes 109

5.1 Introduction . . . . . . . . . . . . . . . . . . . . 110

5.2 Geometrical description and design variables . . . . . . . . . . . . . 112

$5.3 \quad$ Coupled shape and topology optimization approach. . . . . . . . . . . 115

5.3 .1 General framework . . . . . . . . . . . . . . . . . 115

$5.3 .2 \quad$ Monolithic and staggered update schemes . . . . . . . . . . 116

5.3 .3 Sensitivity analysis . . . . . . . . . . . . . . . . . 118

5.3.4 Update of design variables . . . . . . . . . . . . . . . . . . . . 120

5.4 Numerical examples . . . . . . . . . . . . . . . . 120

5.4.1 Circular and square thin-walled cantilever beams . . . . . . . . 120

5.4 .2 HAWT rotor blade . . . . . . . . . . . . . . . . . . . . . . . . . . . . . . . 127

5.5 Conclusions . . . . . . . . . . . . . . . . . . 132

$\begin{array}{llr}6 & \text { Conclusions and recommendations } & 137\end{array}$

6.1 Conclusions . . . . . . . . . . . . . . . . 137

6.1 .1 Coupled shape and topology optimization method . . . . . . . 137

6.1 .2 Coupled optimization of beam-type structures. . . . . . . . . . 138

$6.1 .3 \quad$ Aerostructural design of turbine blades . . . . . . . . . . . . . 139

6.1 .4 Comparison of numerical update schemes . . . . . . . . . . . . 140

6.2 Recommendations . . . . . . . . . . . . . . . . . . 141

\begin{tabular}{ll}
\hline Bibliography & 143
\end{tabular}

\begin{tabular}{ll}
\hline List of publications & 157
\end{tabular} 
Contents

Curriculum Vitae 159 


\section{Chapter 1}

\section{Introduction}

\subsection{Problem statement}

Emission of greenhouse gases are the leading cause for the earth's rapidly changing climate [1, 2]. One of the most effective strategies to mitigate this effect is to decarbonize the generation of electricity [3, which can be accomplished by using renewable resources, such as wind energy, solar energy, hydropower, geothermal power and biomass energy [4. Among these possibilities, the application of wind energy grows fast in popularity and currently accounts for about $50 \%$ of the renewable resources [5], which is the result of its technological maturity, good infrastructure, and relatively low expenses [6, 7]. Also in the near future wind energy is expected to play an important role in the generation of green electricity, due to increasing financial investments in sustainable resources [8,11] and the continuous growth in energy demand [5, 12].

The Horizontal-Axis Wind Turbine (HAWT) is the most common configuration among existing wind energy converters [13, 14. Its theoretical power output may be formulated as [15]

$$
P=\frac{1}{2} C_{\mathrm{p}} \rho A U^{3}
$$

where $\rho$ and $U$ are the air (wind) density and speed, respectively, $A=\pi R^{2}$ is the rotor swept area with $R$ being the rotor radius, and $C_{\mathrm{p}}$ is the power coefficient, which reflects how efficiently the wind turbine converts the energy in the wind to electricity. The power coefficient has a theoretical maximum value of 0.593 , known as the Betz limit [16, which means no turbine can capture more than $59.3 \%$ of the available kinetic energy in the wind.

As illustrated by the cubic relation Eq. (1.1), the wind speed $U$ has the most dominant effect on the turbine power output $P$. However, the location [17-19] and layout [20 22] of a wind farm need to be determined adequately to guarantee abundant 
wind resource for each standalone turbine. In addition, the wind speed generally increases with the height above the ground [15]. Hence, increasing the hub height (i.e., the rotor's height above the ground) together with the rotor swept area $A$ typically leads to an effective increase of the wind turbine's power output [23, and explains why commercial wind turbines have continuously grown in size over the last 40 years [6, 13, see Figure 1.1 Currently, $8 \mathrm{MW}$ wind turbines with blade lengths of $88 \mathrm{~m}$ or more are available on the market, which correspond to a rotor swept area large enough to cover 3 soccer fields 24. Although a further upscaling of the rotor size enables achieving an even higher turbine capacity, it introduces several important challenges. First, the rotor mass grows faster than its power output, because the mass (or volume) and power output of a turbine rotor are proportional to the cube and square of the blade length, respectively (which is known as the square-cube law) [15. In addition, a larger mass leads to a higher material demand and increases the mass-induced loads. The latter aspect imposes stringent requirements on the support structure (tower, foundation) of the wind turbine, thereby increasing the manufacturing costs [25]. Furthermore, the sensitivity to aeroelastic instabilities can grow, since a larger rotor induces larger aerodynamic and inertia forces [26, 27.

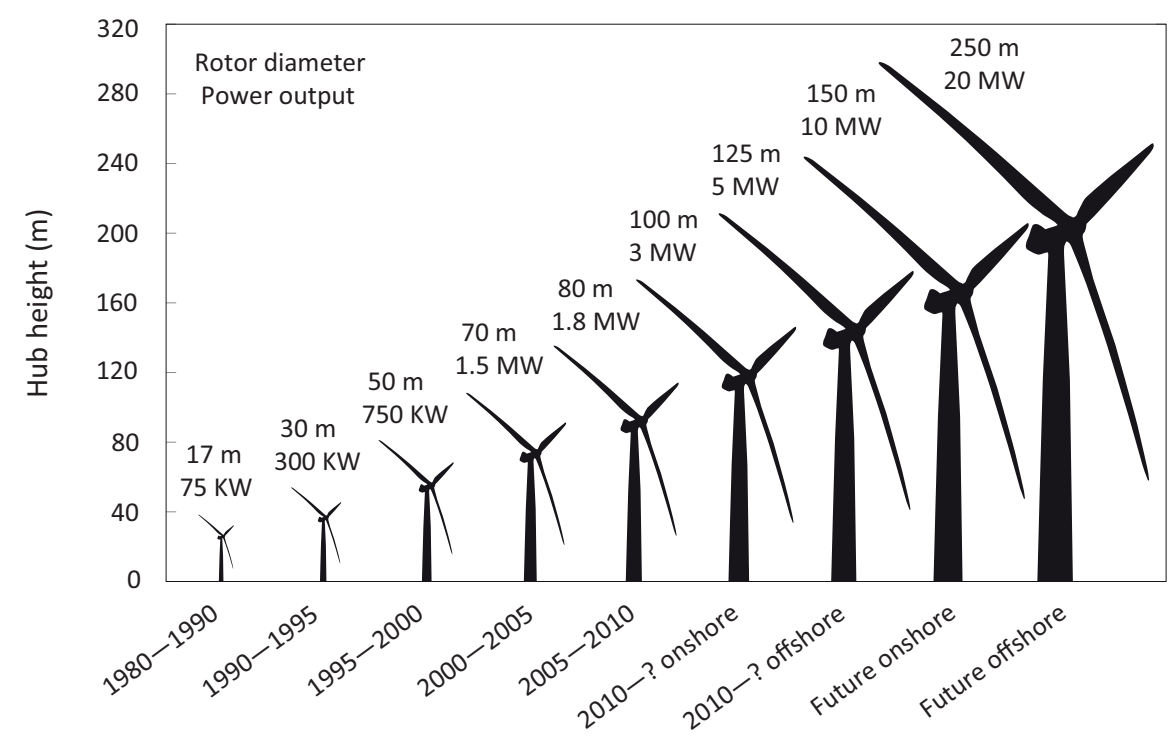

Figure 1.1: Current trend in wind turbine size [28].

Fortunately, the above issues in wind turbine upscaling can be alleviated by means of new, innovative designs of wind turbine rotor blades. For example, the use of new materials makes it possible to modify the square-cube law mentioned above, i.e., it has been confirmed that the exponent associated with the growth of blade mass as a function of the blade length may become lower than 3 for various composite materials 29 31]. Moreover, the operational performance of a rotor can be improved 
through adapting the blade geometry design. The blade geometry of a commercial HAWT typically is represented by a hollow airfoil that is reinforced by spar caps and shear webs [15], as shown in Figure 1.2 (a), which enables the generation of an efficient aerodynamic lift force as a result of the pressure difference across the airfoil [32. In specific, the shape of the turbine blade can be optimally designed with respect to the aerodynamic characteristics, such as the rotor power coefficient $C_{\mathrm{p}}$ present in Eq. (1.1) and the induced aerodynamics forces 33 35. Since a change of the airfoil geometry also affects the structural properties, i.e., the blade stiffness and mass, the optimized blade design follows from a compromise of the aerodynamic and structural requirements, leading to the typical shape shown in Figure $1.2(\mathrm{~b})$.

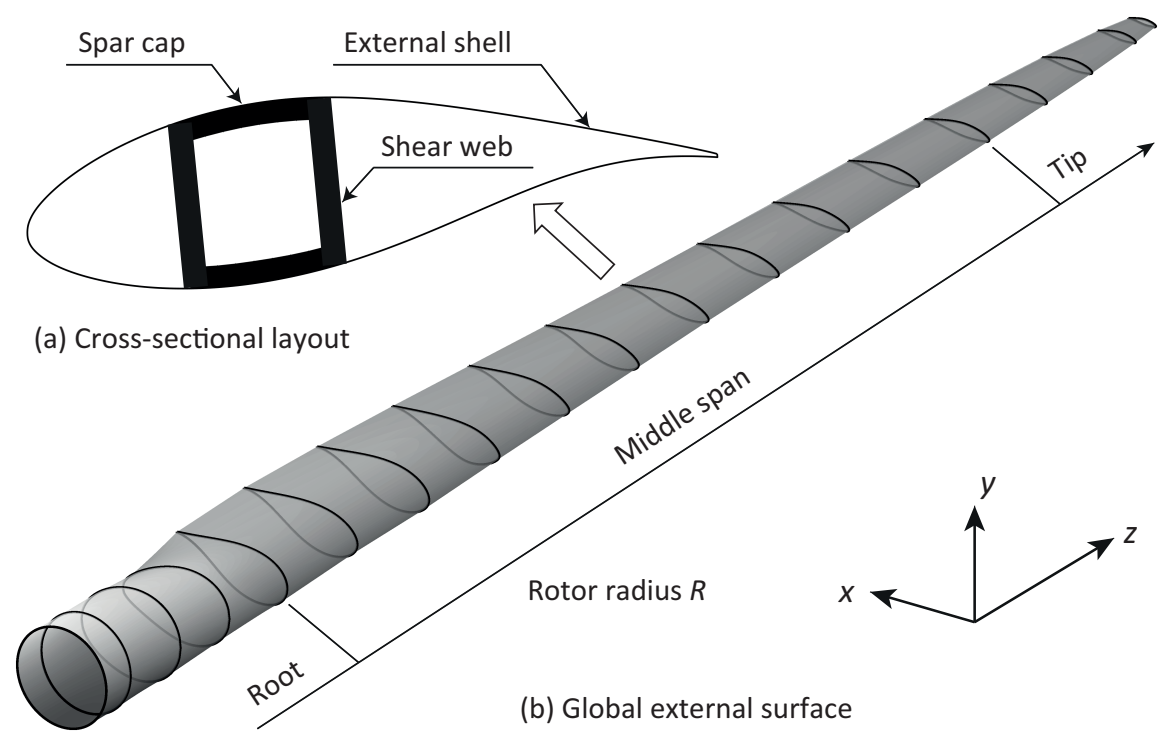

Figure 1.2: Geometry of blade (a) cross-sectional layout and (b) global external surface.

In order to continue wind turbine upscaling in the coming years, the design of the blade geometry needs to be further optimized to achieve a higher rotor power output and a greater blade stiffness, thereby also accounting for a weight reduction. This, however, is a challenging task because these three objectives are commonly in conflict with each other. Maximizing the power extracted from the wind usually is accompanied by an increase of the blade mass [36, 37] and the aerodynamic forces induced [38. Consequently, the blade deflections increase [39, which in turn puts more stringent requirements on the blade stiffness. Furthermore, the use of less material is in contradiction with the goal of reaching a higher blade stiffness when the material distribution is kept fixed [40, 41. In order to systematically overcome these complexities, optimization techniques have recently drawn increased attention in the design of wind turbine blades 42 .

The three categories that are typically distinguished in a structural optimization process are size, shape and topology optimization. These three techniques address 


\subsection{Problem statement}

different aspects of the structural design problem [43, and for some time have been applied for wind turbine blades. Size optimization parameterizes the dimension of the geometry, and is commonly used for optimizing the outer thickness of the airfoil and the size and location of the reinforcements, see Figure 1.2(a) 44 47. The blade design objectives in size optimization usually relate to the structural performance, e.g., a reduction of the mass or the maximal deflection for a prescribed outer shape of the blade. Conversely, shape optimization is concerned with finding an optimal outer shape of the structure [48, see also Figure 1.2 (b). In the shape optimization of turbine rotor blades, the objective typically refers to the aerodynamic performance, i.e., the rotor power output, so that this approach is also denoted as Aerodynamic Shape Optimization (ASO) [49, 50]. One of the limitations of size and shape optimization is that the internal topological layout is kept fixed during the optimization process. To overcome this aspect, topology optimization methods have been developed, which aim at finding an optimal material distribution for the specific structure under consideration [51, 52. This method starts without an a priori assumption on the structural configuration, and optimizes the overall topology of the structure within a predefined design domain 43. In the design of wind turbine blades, topology optimization is commonly used to explore alternative interior blade layouts or to identify specific types of reinforcement within a prescribed external blade geometry [25, 53. As described above, the possible solutions of the material distribution found with topology optimization are bounded by the specific size and shape of the design domain chosen 54. This limitation can be reduced by choosing the design domain as large as possible, although this may lead to relatively large computational times. Another drawback of a fixed design domain is that it impedes the optimization of aeroelastic structures, such as aircraft wings and wind turbine blades. Since the aerodynamic loading is characterized by the size and shape of the design domain, the design domain of aeroelastic structures needs to be variable in order to optimize their aerodynamic performance.

Due to the limitations of each of the optimization methods discussed above, none of these methods could be individually used to find the best trade-off between the aforementioned three main design objectives for wind turbine blades, which are a higher rotor power output, a greater blade stiffness and a lower weight. Instead, the optimization of the aerostructural performance of wind turbines is usually carried out in two consecutive stages [15, which correspond to the aerodynamic design of the blade outer shape and the structural design of the interior layout of the blade. During the first stage, a shape optimization process is performed, in which the blade length, airfoil profile and chord and twist distributions of the blade are varied to achieve an optimal aerodynamic performance 49, 50, 55, 56. During the second stage, the interior layout of the blade is optimized using the aerodynamic loads from the first stage as input. Traditionally, the optimization process starts from a hollow airfoil, whereby subsequently reinforcements (spar caps and shear webs) are added to improve the bending, shear, and torsional resistances of the blade 57. The internal blade layout is usually optimized by adapting the thickness of the airfoil and the size and location of the reinforcements via size and shape optimization methods 45.47. However, this strategy does not naturally allow for the introduction of new topological concepts for the blade layout, which is the reason that topology optimization techniques have been 
applied to find the optimal shapes and locations for the stiffeners of the turbine blades 53 .

Since the shape and topology optimization processes typically are performed in two consecutive stages, the coupling effects between the blade aerodynamics and its structural performance are not adequately taken into account. The aerostructural design and multi-objective optimization techniques presented in [35, 39, 58, 60, could overcome this limitation, but these frameworks so far have only been applied to specific blade layouts for which the outer shape is optimized together with the size and location of the stiffeners. In addition, the update of the design variables for most of the optimization techniques is performed with gradient-free optimization algorithms, such as genetic algorithms [36, 41, 59, 60. These algorithms facilitate an easy and flexible numerical implementation [37. and the possibility of finding a global optimum, but may also exhibit a poor convergence behavior [36] and thus are computationally expensive, especially if the number of design variables is relatively large [61. From these viewpoints, gradient-based optimization methods, for which the sensitivities are derived in analytical form, are preferable. In accordance with the state of the art and challenges described above, in this thesis it is explored how size, shape and topology optimization can be efficiently integrated within a single modeling framework in order to simultaneously optimize the outer shape and interior topological layout of wind turbine rotor blades from aerodynamic and structural requirements. The research objectives and methodology are described in detail in the section below.

\subsection{Research objectives and methodology}

The research presented in this thesis aims at the development of a gradient-based, coupled aerostructural shape and topology optimization framework, which should simultaneously optimize the external shape and interior layout of HAWT rotor blades from both aerodynamic and structural requirements. This optimization framework can be used as a practical tool for optimizing the geometrical design of rotor blades, such that the overall costs of wind energy are reduced.

For meeting the main research goal above, the following methodology is applied:

1. A coupled shape and topology optimization model is formulated based on incorporating the shape design variables of the design domain in the SIMP (Solid Isotropic Material with Penalization) topology optimization method. This approach enables a shape variation of the design domain in a topology optimization process.

2. Non-Uniform Rational B-Splines (NURBS) are employed to parameterize the shape of the design domain, which guarantees a highly accurate description of the shape boundaries and enable an efficient control of the design domain with only a few control points.

3. The beam Finite Element Method (FEM) and Blade Element Momentum (BEM) models are used to determine the aerostructural optimization of the rotor blade 
in a computationally efficient manner. Since a wind turbine blade is represented by a beam-type structure, in the FEM formulation the coupled optimization framework is specified towards $2.5 \mathrm{D}$ and $3 \mathrm{D}$ beam models. In the $2.5 \mathrm{D}$ beam model, standard beam elements are employed to simulate the structural response in the longitudinal direction, whereby the cross-sectional properties of the beam element are calculated from additional 2D FEM analyses. The results obtained from a coupled optimization analysis of the $2.5 \mathrm{D}$ beam model will be compared to those computed for a 3D beam model, which should demonstrate the accuracy and computational efficiency of the $2.5 \mathrm{D}$ formulation.

4. The shape and topology sensitivities of the above FEM-BEM model are derived in closed form, which enables the use of a gradient-based optimization algorithm that further improves the computational efficiency.

5. The coupled shape and topology optimization model is solved using staggered and monolithic update schemes. The numerical efficiency and the solution obtained by these update schemes is compared in detail in order to establish a solid confidence in the results calculated by the coupled shape and topology optimization method.

6. The usefulness and advantage of the presented coupled optimization framework is demonstrated by investigating several benchmark problems, as well as practical case studies related to the aerostructural design of a reference HAWT rotor blade.

\subsection{Thesis outline}

Chapter 2 reviews existing shape and topology optimization techniques. The widely used density-based topology optimization method is combined with a NURBS-based shape optimization method to build a 2D sequentially coupled domain shape and topology optimization framework. The modeling framework is applied for the analysis of several benchmark problems. The structural designs obtained for these benchmark problems are compared to those computed by pure topology optimization, which demonstrates the potential of the coupled optimization method in finding interesting, alternative structural solutions.

Chapter 3 presents a coupled shape and topology optimization framework for $2.5 \mathrm{D}$ and 3D beam models, which are representative for rotor blades used in HAWTs. The governing equations for the coupled shape and topology framework are derived, and a comparison study is performed that demonstrates the accuracy and computational efficiency of the $2.5 \mathrm{D}$ beam model with respect to the $3 \mathrm{D}$ beam model.

On the basis of the computationally efficient 2.5D coupled optimization framework presented in Chapter 3, in Chapter 4 a Coupled Multi-objective Shape and Topology Optimization (CMSTO) approach is formulated for the aerostructural design of HAWT rotor blades. The aerostructural behavior of the rotor blade is simulated using BEM and beam FEM models, whereby in the optimization approach the rotor power coefficient and the structural compliance of the blade are considered as the two sub-objectives. The practical usefulness of the coupled optimization method is 
demonstrated by optimizing a NREL 5MW reference rotor blade, whereby the results are compared to those obtained by a separate Aerodynamic Shape Optimization and Structural Topology Optimization approach (ASO-STO), as well as a pure Structural Topology Optimization (STO) approach.

In Chapters 2 to 4 the coupled shape and topology optimization framework is numerically solved using a staggered update scheme. For investigating the effect of the solution strategy on the results calculated by the coupled optimization framework, in Chapter 5 the results computed with the staggered update scheme are compared to those calculated by a monolithic update scheme that updates the shape and topology design variables in a concurrent fashion. The numerical examples in the comparison study concern basic circular and square cantilever beam structures, as well as a more complex, non-prismatic beam structure representative of a rotor blade used in a HAWT.

Finally, Chapter 6 completes this thesis by summarizing the main conclusions of the research and recommending possible topics for future research.

Note that Chapters 2 to 5 are based on journal papers, and each chapter is presented as such that it can be read separately. 


\section{Chapter 2}

\section{Coupled shape and topology optimization method}

This chapter is based on the journal paper:

Wang, Z., Suiker, A. S. J., Hofmeyer, H., Kalkman, I., Blocken, B., 2020. Sequentially coupled gradient-based topology and domain shape optimization. Optimization and Engineering, 34 pages, https://doi.org/10.1007/s11081-020-09546-3

A coupled topology and domain shape optimization framework is presented that is based on incorporating the shape design variables of the design domain in the SIMP (Solid Isotropic Material with Penalization) topology optimization method. The shape and topology design variables are incrementally updated in a sequential fashion, using a staggered numerical update scheme. Non-Uniform Rational B-Splines (NURBS) are employed to parameterize the shape of the design domain. This not only guarantees a highly accurate description of the shape boundaries by means of smooth basis functions with compact support, but also enables an efficient control of the design domain with only a few control points. Furthermore, the optimization process is performed in a computationally efficient way by applying a gradient-based optimization algorithm, for which the sensitivities can be computed in closed form. The usefulness of the coupled optimization approach is demonstrated by analyzing several benchmark problems that are subjected to different types of initial conditions and domain bounds. The variation in simulation results denotes that a careful construction of the initial design domain is necessary and meaningful. 


\subsection{Introduction}

Topology optimization is a mathematical method that optimally places the material within a given design domain for the specific loading and boundary conditions applied. It has been recognized as the most active research area in the field of structural optimization over the past two decades [52]. Driven by a major interest from both academia and industry, various topology optimization approaches have been developed [51, 62 65. The density-based methods, especially the so-called SIMP (Solid Isotropic Material with Penalization) method [43, 66], are the most widely used techniques. They start from a discretization of the prescribed design domain into a finite element model, whereby a design variable, which is the relative density, is assigned to each finite element. The relative density can take values between zero (a void) and unity (a solid), and is directly related to the element stiffness via a penalization factor. Accordingly, the optimized distribution of the relative density can be determined by adopting an optimization algorithm that computes the minimal structural compliance (which corresponds to the maximal structural stiffness) of the design domain, see also 67-70 and references therein.

Due to the nature of the SIMP method, however, the possible solutions of the density distribution are bounded by the specific size and shape of the design domain chosen. This limitation can be reduced by choosing the design domain as large as possible, although this may lead to relatively large computational times. Alternatively, the construction of the design domain may be formulated as an optimization problem 54. Such a strategy not only offers the possibility to keep computational times more manageable, it also facilitates the optimization of aeroelastic structures, such as wind turbine blades [53] and aircraft wings [71, whereby the applied loads (i.e., the aerodynamic forces) are characterized by the actual size and shape of the design domain.

Previous research has demonstrated that the variation of the design domain within an optimization strategy can be accomplished by means of several approaches. A first possible approach is based on the so-called design space optimization method [54, 72, which adjusts the design domain boundary during topology optimization by adding new design elements. This approach seems to be relatively straightforward to implement, but has the drawback that the boundaries of the domain are represented by a jagged geometry characterized by the element boundaries of the finite element discretization. A second approach that enables the variation of the design domain is the level-set method [73. As demonstrated in [74-77, this method allows to elegantly combine shape and topology optimizations within a single framework. In comparison to density-based topology optimization methods, with the level-set method it is more difficult to mathematically describe the appearance of holes of arbitrary shape inside the design domain [78. In addition, level-set functions may become too flat or too steep during the optimization procedure, such that they need to be periodically reinitialized in order to obtain a solution with sufficient numerical accuracy, which increases the computational cost. An approach somewhat similar to the levelset method is the phase-field method, which uses a partial differential equation for describing the evolution of topologies in a structural optimization problem [79, 80]. 
Although the topologies are solved over the complete design domain without the need of prior information on the location of phase boundaries, the phase-field method has a comparable design space as the SIMP method, whereby the optimized solution depends on the initial shape selected [81. Additionally, in the Moving Morphable Components (MMC) method [82, 83, the topology optimization is performed by adapting the size and shape of a set of morphable structural components. In contrast to the classical SIMP method, however, the MMC method does not facilitate the generation of holes, which thus limits the range of possible topological solutions.

In order to avoid the jagged geometrical boundaries typically resulting from optimizing the topology of a discretized finite element model with the SIMP method, in [84 87] the structural layout calculated with the SIMP method is smoothed using spline functions. Apart from improving the quality of the optimization result, this adaptation also increases the computational speed of the SIMP approach by reducing the number of active optimization variables. In [88] this so-called adaptive topology optimization technique has been subsequently extended towards a combined shape and topology approach for shell structures, whereby the design domain is defined by Bsplines and their variability is accounted for by modifying the positions of the control points. The determination of the shape sensitivities is performed numerically using a finite difference method, which, although computationally demanding, has shown to lead to stable, converged results.

Along the lines of [88, in the present communication a coupled domain shape and topology optimization method is formulated by incorporating the shape design variables of the design domain in the SIMP topology optimization method. However, the formulation is generalized by considering design domains of arbitrary shape. The shape and topology design variables are incrementally updated in a sequential fashion, using a so-called staggered numerical update scheme. Non-Uniform Rational B-Splines (NURBS) are employed to parameterize the shape of the design domain [89. This not only guarantees a highly accurate description of the shape boundaries by means of smooth basis functions with compact support, but also enables an efficient control of the design domain with only a few control points [48. Further, the optimization process is performed in a computationally efficient way by applying a gradient-based optimization algorithm, for which the sensitivities can be computed in closed form; this is another important difference compared to the approach advocated in [88. The objective function adopted in the coupled optimization method refers to the structural compliance, which is the common objective used in topology optimization. However, if required, it is relatively straightforward to replace this objective function in the formulation by an alternative choice, or by a combination of various objective functions, in accordance with a multi-objective optimization approach. Minimizing the structural compliance under the constraint of a significant weight reduction by searching for optima regarding the shape as well as the topology certainly has significant practical value. As will be shown through various numerical examples, it may lead to substantially different structural designs compared to when this optimization is performed on the topology only, thus offering interesting, alternative structural solutions. The main features of the coupled optimization method are demonstrated by means of illustrative $2 \mathrm{D}$ benchmark problems. 
This chapter is organized as follows. Section 2.2 introduces the basic features of the coupled topology and domain shape optimization framework, by starting from the classical formulation for topology optimization. The minimization problem is formulated and the staggered numerical update scheme is described. Section 2.3 reviews the mathematical description of the geometry of the design domain by means of NURBS. In Section 2.4 the Finite Element Method (FEM) formulation for the coupled optimization formulation is summarized. The FEM formulation forms the basis for the computation of the sensitivities of the structural compliance to the shape and topology design variables, which is presented in Section 2.5 together with a description of the update of the design variables. Section 2.6 treats several numerical benchmark problems that demonstrate the basic features of the coupled optimization method. Finally, in Section 2.7 some concluding remarks are given.

\subsection{Problem formulation and solution strategy}

In accordance with the SIMP approach, topology optimization can be mathematically formulated by means of the following minimization problem [43, 66]:

$$
\begin{aligned}
\min _{\boldsymbol{\rho}} & c(\boldsymbol{\rho})=\mathbf{f}^{\mathrm{T}} \mathbf{u}(\boldsymbol{\rho}), \\
\text { subject to } & \frac{V(\boldsymbol{\rho})}{V_{0}}=f_{r}, \\
\text { with } & 0<\rho_{\min } \leq \rho^{e} \leq 1, \quad e=1, \ldots, N,
\end{aligned}
$$

where the objective function $c$ typically is referred to as the structural compliance, $\rho$ is the vector of design variables that is composed of the relative densities $\rho^{e}$ of the elements $e, \rho_{\text {min }}$ is the prescribed minimum density (which is non-zero to avoid a singular stiffness matrix), $N$ is the total number of elements, $V(\boldsymbol{\rho})$ and $V_{0}$ represent the current (material) volume and the initial volume of the design domain, respectively, and $f_{r}$ is the volume fraction of material. Further, $\mathbf{f}$ and $\mathbf{u}$ are the global force and displacement vectors, respectively, which are related via

$$
\mathbf{f}=\mathbf{K u}
$$

with $\mathbf{K}$ the global stiffness matrix.

The formulation given by Eq. 2.1) refers to a fixed outer shape of the design domain. To allow for a variable outer shape, Eq. 2.1) is extended as

$$
\begin{array}{rll}
\min _{\boldsymbol{\rho}, \mathbf{a}} & c(\boldsymbol{\rho}, \mathbf{a})=\mathbf{f}^{\mathrm{T}} \mathbf{u}(\boldsymbol{\rho}, \mathbf{a}), \\
\text { subject to } & \frac{V(\boldsymbol{\rho}, \mathbf{a})}{V_{0}}=f_{r}, \\
\text { with } & l_{m} \leq a_{m} \leq u_{m}, & m=1, \ldots, M, \\
& 0 \leq \rho_{\text {min }} \leq \rho^{e} \leq 1, \quad e=1, \ldots, N,
\end{array}
$$


where $\mathbf{a}$ is the vector of shape-related design variables with the elements $l_{m}$ and $u_{m}$ representing the lower and upper bounds of the design variable $a_{m}$, and $M$ is the total number of shape-related design variables. Note that in Eq. (2.3) the material volume $V$ is a function of both the shape-related design variables a and the density variables $\boldsymbol{\rho}$, and that the initial volume of the design domain $V_{0}$ corresponds to the initial shape.

The formulation given by Eq. (2.3) can be solved by updating the shape variables a and density variables $\rho$ in parallel using a so-called monolithic scheme, or by performing the update of the shape and density variables in a sequential fashion using a staggered scheme. In this work a staggered update scheme is used, which at a specific time step keeps the shape variables a momentarily fixed while solving for the density variables $\boldsymbol{\rho}$ in accordance with the topology optimization formulation given by Eq. 2.1. Subsequently, the density variables $\boldsymbol{\rho}$ are frozen, and the shape variables a are optimized by solving the minimization problem:

$$
\begin{array}{rl}
\min _{\mathbf{a}} & c(\mathbf{a})=\mathbf{f}^{\mathrm{T}} \mathbf{u}(\mathbf{a}), \\
\text { subject to } & \frac{V(\mathbf{a})}{V_{0}}=f_{r}, \\
\text { with } & l_{m} \leq a_{m} \leq u_{m}, \quad m=1, \ldots, M .
\end{array}
$$

This sequence of optimization steps is repeated until both optimization formulations have reached their convergence criterion. Figure 2.1 shows the flowchart of the staggered solution scheme. The first step is to construct a geometry description of the design domain to be analyzed, which is done by decomposing the design domain into design elements and determining the coordinates of the control points of these elements. Subsequently, a finite element model is constructed by meshing the design elements. The relative densities $\rho$ of the finite elements are optimized by solving Eq. (2.1), which is done by performing a structural analysis, a topology sensitivity analysis, and a density update procedure. Accordingly, the optimized relative density distribution is found for the design domain (for which the shape has been momentarily kept fixed). The next step is to optimize the shape of the design domain, based on the optimized densities of the finite elements just computed in the topology optimization step. This step is carried out by solving Eq. (2.4), in which a structural analysis, a shape sensitivity analysis, and a shape update procedure are performed. When the relative element densities computed in the last topology optimization step are not optimal for the new shape, a new topology optimization step needs to be conducted based on the new shape. The above sequence of steps is repeated until the stop criteria for both topology optimization and domain shape optimization are satisfied. Note that the solution strategy in Figure 2.1 starts with topology optimization and may therefore be referred to as Topology Shape Optimization (TSO). Alternatively, it can start with domain shape optimization, and will then be referred to as Shape Topology Optimization (STO).

Although the element densities are kept fixed during an incremental shape optimization step, the overall density distribution, and thus the internal topology, changes 
as a result of a change in the nodal coordinates of the finite elements. However, the shape changes during an incremental shape optimization step are such that the corresponding alterations in the topology typically are relatively small and thus acceptable. Moreover, possible inaccuracies generated by these topology alterations may be expected to vanish in the subsequent incremental topology optimization step.

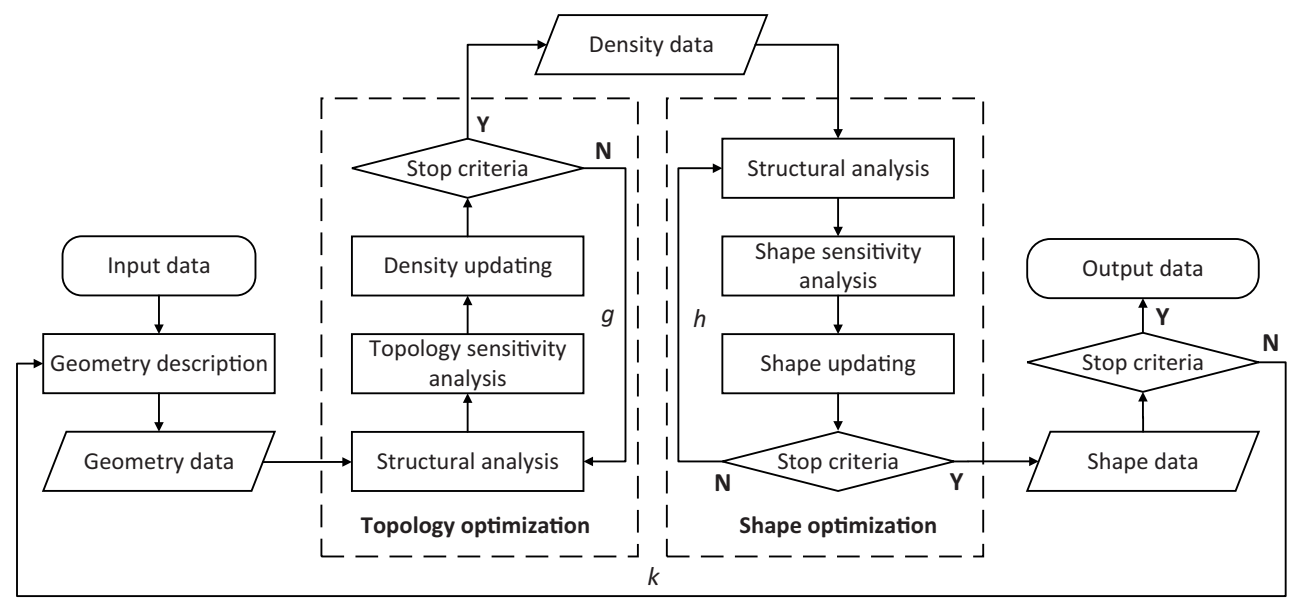

Figure 2.1: Flowchart of the staggered solution scheme for coupled topology and domain shape optimization with iterations $g$ and $h$ referring to topology and shape optimization subloops, respectively, and iteration $k$ referring to the outer loop.

\subsection{Geometry description}

As visualized in Figure 2.2 the description of the geometry of the design domain $\Omega$ can be obtained from the parametric domain $\boldsymbol{\Omega}_{0}$ using a geometry function $\mathbf{G}$, see also 90. Accordingly, for an arbitrary point $(\xi, \eta)$ in the parametric domain $\boldsymbol{\Omega}_{0}$, the corresponding point $(x, y)$ in the physical design domain $\boldsymbol{\Omega}$ is computed via the projection

$$
(x, y)=\mathbf{G}(\xi, \eta)
$$

with the geometry function $\mathbf{G}(\xi, \eta)$ defined by a set of basis functions and control points in the physical design domain. Observe from Figure 2.2 that the geometry function $\mathbf{G}$ projects the fixed auxiliary mesh defined in the parametric domain to an adequate finite element mesh for the actual design domain, see also [91. The geometry function $\mathbf{G}$ is here constructed by NURBS, which have the advantage that they can describe a large variety of relatively complex geometries with high accuracy [92. Since NURBS are based on B-splines, these will be reviewed first for reasons of clarity. 

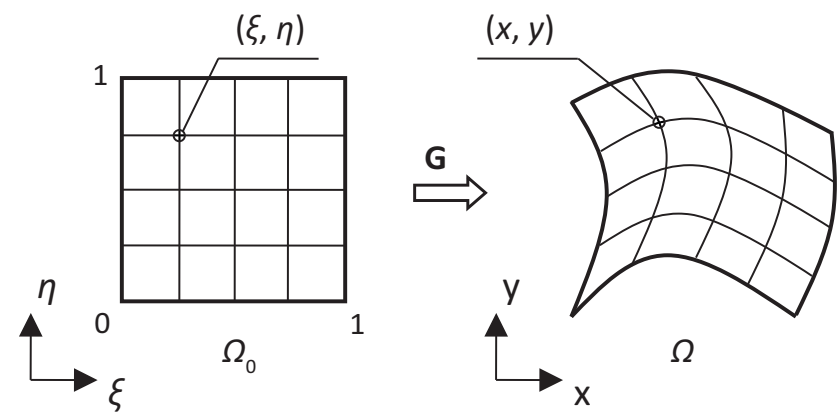

Figure 2.2: Parameterization of the physical design domain $\boldsymbol{\Omega}$ via a coordinate projection with a geometry function $\mathbf{G}$ connected to the parametric domain $\boldsymbol{\Omega}_{0}$. The solid lines in the parametric domain $\boldsymbol{\Omega}_{0}$ define a fixed auxiliary mesh, which is projected through $\mathbf{G}$ to the actual finite element mesh that discretizes the design domain.

\subsubsection{B-spline surface}

For constructing a B-spline surface, the coordinates in the two-dimensional parametric domain are assembled in a non-decreasing fashion by using so-called knot vectors [89]

$$
\begin{aligned}
& \boldsymbol{\Xi}=\left[\xi_{1}, \xi_{2}, \ldots, \xi_{s+p+1}\right]^{\mathrm{T}}, \\
& \boldsymbol{\Theta}=\left[\eta_{1}, \eta_{2}, \ldots, \eta_{t+q+1}\right]^{\mathrm{T}} \quad \text { with } \quad \xi_{i}, \eta_{j} \in \mathbb{R},
\end{aligned}
$$

where $i, j$ are the knot indices, $s$ and $t$ are the number of control points (and thus the number of basis functions used for constructing the B-spline surface) in the $x$ - and $y$-directions, and $p, q$ are integers defining the polynomial orders of the basis functions. The B-spline basis functions $S_{i, p}(\xi)$ in the $\xi$-direction can be obtained recursively as

$$
\begin{aligned}
\text { for } \quad p=0: S_{i, 0}(\xi)= & \begin{cases}1 & \text { if } \xi_{i} \leq \xi<\xi_{i+1}, \\
0 & \text { otherwise }\end{cases} \\
\text { for } \quad p \geq 1: S_{i, p}(\xi)= & \frac{\xi-\xi_{i}}{\xi_{i+p}-\xi_{i}} S_{i, p-1}(\xi) \\
& +\frac{\xi_{i+p+1}-\xi}{\xi_{i+p+1}-\xi_{i+1}} S_{i+1, p-1}(\xi)
\end{aligned}
$$

with the index $i$ running from 1 to the number of control points $s$. The B-spline basis functions $T_{j, q}(\eta)$ in the $\eta$-direction can be determined in a similar fashion as shown in Eq. 2.7). The control points are defined by vectors including their $x$ - and $y$-coordinates, which construct the geometry in the physical design domain via a twodimensional control net $\mathbf{C}_{i, j}$, with $i=1,2, \ldots s, j=1,2, \ldots t$. The B-spline surface follows from combining the control net with the tensor product of the basis functions $S_{i, p}(\xi)$ and $T_{j, q}(\eta)$ as 


$$
\mathbf{B}(\xi, \eta)=\sum_{i=1}^{s} \sum_{j=1}^{t} S_{i, p}(\xi) T_{j, q}(\eta) \mathbf{C}_{i, j} .
$$

Accordingly, the B-spline surface $\mathbf{B}(\xi, \eta)$ may represent the geometry function $\mathbf{G}(\xi, \eta)$ indicated in Figure 2.2 .

\subsubsection{NURBS surface}

From the B-spline basis functions $S_{i, p}(\xi)$ and $T_{j, q}(\eta)$, a rational surface $R_{i, j}(\xi, \eta)$ can be constructed as 92

$$
R_{i, j}(\xi, \eta)=\frac{S_{i, p}(\xi) T_{j, q}(\eta) \omega_{i, j}}{\sum_{\hat{i}=1}^{s} \sum_{\hat{j}=1}^{t} S_{\hat{i}, p}(\xi) T_{\hat{j}, q}(\eta) \omega_{\hat{i}, \hat{j}}},
$$

where $\omega_{i, j}$ are the weights of the control points. The rational surface can be subsequently combined with the control net $\mathbf{C}_{i, j}$, to obtain the NURBS surface:

$$
\mathbf{G}(\xi, \eta)=\sum_{i=1}^{s} \sum_{j=1}^{t} R_{i, j}(\xi, \eta) \mathbf{C}_{i, j}
$$

Using matrix-vector notation, Eq. 2.9 can be compactly written as

$$
R_{i, j}(\xi, \eta)=\frac{S_{i, p}(\xi) T_{j, q}(\eta) \omega_{i, j}}{\mathbf{S}^{\mathrm{T}} \mathbf{W} \mathbf{T}}
$$

with

$$
\mathbf{S}=\left[S_{1, p}(\xi), S_{2, p}(\xi), \cdots, S_{s, p}(\xi)\right]^{\mathrm{T}}
$$

and

$$
\mathbf{W}=\left[\begin{array}{cccc}
\omega_{1,1} & \omega_{1,2} & \cdots & \omega_{1, t} \\
\omega_{2,1} & \omega_{2,2} & \cdots & \omega_{2, t} \\
\vdots & \vdots & \ddots & \vdots \\
\omega_{s, 1} & \omega_{s, 2} & \cdots & \omega_{s, t}
\end{array}\right]
$$

and

$$
\mathbf{T}=\left[T_{1, q}(\eta), T_{2, q}(\eta), \cdots, T_{t, q}(\eta)\right]^{\mathrm{T}}
$$

With these expressions, the NURBS surface given by Eq. 2.10 becomes 


$$
\mathbf{G}(\xi, \eta)=\frac{1}{\mathbf{S}^{\mathrm{T}} \mathbf{W} \mathbf{T}} \sum_{i=1}^{s} \sum_{j=1}^{t} S_{i, p}(\xi) T_{j, q}(\eta) \omega_{i, j} \mathbf{C}_{i, j}
$$

Since the basis functions $S_{i, p}(\xi)$ and $T_{j, q}(\eta)$ form a partition of unity, the NURBS surface, Eq. 2.15), reduces to the B-spline surface, Eq. 2.8), when all weights $\omega_{i, j}$ are set equal to unity 92 .

The control points with their corresponding weights can be conveniently stored in the matrices $\mathbf{X}$ and $\mathbf{Y}$ as

$$
\mathbf{X}=\left[\begin{array}{cccc}
\omega_{1,1} X_{1,1} & \omega_{1,2} X_{1,2} & \cdots & \omega_{1, t} X_{1, t} \\
\omega_{2,1} X_{2,1} & \omega_{2,2} X_{2,2} & \cdots & \omega_{2, t} X_{2, t} \\
\vdots & \vdots & \ddots & \vdots \\
\omega_{s, 1} X_{s, 1} & \omega_{s, 2} X_{s, 2} & \cdots & \omega_{s, t} X_{s, t}
\end{array}\right]
$$

and

$$
\mathbf{Y}=\left[\begin{array}{cccc}
\omega_{1,1} Y_{1,1} & \omega_{1,2} Y_{1,2} & \cdots & \omega_{1, t} Y_{1, t} \\
\omega_{2,1} Y_{2,1} & \omega_{2,2} Y_{2,2} & \cdots & \omega_{2, t} Y_{2, t} \\
\vdots & \vdots & \ddots & \vdots \\
\omega_{s, 1} Y_{s, 1} & \omega_{s, 2} Y_{s, 2} & \cdots & \omega_{s, t} Y_{s, t}
\end{array}\right]
$$

where $X_{i, j}, Y_{i, j}$ (with $i=1,2, \cdots, s ; j=1,2, \cdots, t$ ) are the control point coordinates. Hence, the projection, Eq. 2.5), of coordinates from the parametric domain $\boldsymbol{\Omega}_{0}$ to the physical design domain $\boldsymbol{\Omega}$ can be reformulated as

$$
(x, y)=\left(\frac{\mathbf{S}^{\mathrm{T}} \mathbf{X T}}{\mathbf{S}^{\mathrm{T}} \mathbf{W} \mathbf{T}}, \frac{\mathbf{S}^{\mathrm{T}} \mathbf{Y} \mathbf{T}}{\mathbf{S}^{\mathrm{T}} \mathbf{W} \mathbf{T}}\right) .
$$

With Eq. 2.18), a surface in the physical design domain thus is completely determined once the knot vectors and control points with their weights are defined.

\subsection{Structural analysis}

In order to solve an optimization problem with the finite element method, the design domain needs to be meshed by finite elements. This is done by creating a fixed auxiliary mesh through defining the finite element node locations in the parametric domain, see also 91, and subsequently mapping these node locations to the actual physical design domain using Eq. 2.18), see Figure 2.2. This enables the construction of an explicit relationship between the shape design variables (represented by the locations of the NURBS control points) and the FEM mesh, so that the FEM mesh is automatically adapted for changes of the structural boundary caused by shape optimization.

The main equations of the finite element method are reviewed below. These equations are subsequently used for deriving a gradient-based optimization framework, by 
determining the sensitivities of the objective function with respect to the shape and topology design variables. Hence, for an individual finite element, the element stiffness matrix $\mathbf{k}^{e}$ is given by

$$
\mathbf{k}^{e}=\int_{-1}^{1} \int_{-1}^{1}\left(\mathbf{B}^{e}\right)^{\mathrm{T}} \mathbf{D}^{e} \mathbf{B}^{e}\left|\mathbf{J}^{e}\right| d \xi^{e} d \eta^{e}
$$

where $\mathbf{B}^{e}$ is the strain-displacement matrix, $\mathbf{D}^{e}$ is the constitutive matrix, $\left|\mathbf{J}^{e}\right|$ is the determinant of the Jacobian matrix $\mathbf{J}^{e}$, and $\xi^{e}$ and $\eta^{e}$ are the parametric coordinates of the finite element shape functions (not to be confused with the parametric coordinates of the geometry function presented in the previous section). The stiffness matrix is calculated by means of numerical integration using Gauss quadrature, i.e.,

$$
\mathbf{k}^{e}=\sum_{i^{*}=1}^{t^{*}} \sum_{j^{*}=1}^{t^{*}} w_{i^{*}} w_{j^{*}}\left(\left(\mathbf{B}^{e}\right)^{\mathrm{T}} \mathbf{D}^{e} \mathbf{B}^{e}\left|\mathbf{J}^{e}\right|\right)_{i^{*}, j^{*}},
$$

where $i^{*}, j^{*}$ are the indices of the integration points, $t^{*}$ equals the number of integration points along each direction, and $w_{i^{*}}$ and $w_{j^{*}}$ are the weighting factors of the integration points. In the present study the two-dimensional optimization problems analyzed are meshed by 4-node quadrilateral plane-stress elements, for which the element strain-displacement matrix $\mathbf{B}^{e}$ present in Eq. 2.20 is composed as

$$
\mathbf{B}^{e}=\left[\begin{array}{llll}
\mathbf{B}_{1}^{e} & \mathbf{B}_{2}^{e} & \mathbf{B}_{3}^{e} & \mathbf{B}_{4}^{e}
\end{array}\right]
$$

with

$$
\mathbf{B}_{n}^{e}=\left[\begin{array}{cc}
\frac{\partial N_{n}}{\partial x} & 0 \\
0 & \frac{\partial N_{n}}{\partial y} \\
\frac{\partial N_{n}}{\partial y} & \frac{\partial N_{n}}{\partial x}
\end{array}\right] \quad \text { with } \quad n \in\{1,2,3,4\}
$$

where $x$ and $y$ represent the spatial directions of the coordinate system in the physical design domain, the index $n$ reflects the node number, and $N_{n}$ represents the corresponding displacement shape function. The 4-node plane-stress elements used in this study are equipped with standard quadratic shape functions:

$$
\begin{aligned}
& N_{1}=0.25\left(1-\xi^{e}\right)\left(1-\eta^{e}\right), \\
& N_{2}=0.25\left(1+\xi^{e}\right)\left(1-\eta^{e}\right), \\
& N_{3}=0.25\left(1+\xi^{e}\right)\left(1+\eta^{e}\right), \\
& N_{4}=0.25\left(1-\xi^{e}\right)\left(1+\eta^{e}\right) .
\end{aligned}
$$


Note that in Eq. (2.22 the spatial derivatives of the shape functions are defined with respect to the spatial coordinates $x$ and $y$ in the physical domain, while in Eq. 2.23 the shape functions are defined by the spatial coordinates $\xi^{e}$ and $\eta^{e}$ in the parametric domain. In order to relate the derivatives of the shape functions in the two domains, the vectors $\mathbf{o}_{n}^{e}$ and $\mathbf{v}_{n}^{e}$ are introduced as

$$
\begin{aligned}
& \mathbf{o}_{n}^{e}=\left[\frac{\partial N_{n}}{\partial \xi^{e}}, \frac{\partial N_{n}}{\partial \eta^{e}}\right]^{\mathrm{T}}, \\
& \mathbf{v}_{n}^{e}=\left[\frac{\partial N_{n}}{\partial x}, \frac{\partial N_{n}}{\partial y}\right]^{\mathrm{T}} \quad \text { with } \quad n \in\{1,2,3,4\} .
\end{aligned}
$$

The relation between the vectors $\mathbf{v}_{n}^{e}$ and $\mathbf{o}_{n}^{e}$ follows as

$$
\mathbf{v}_{n}^{e}=\left(\mathbf{J}^{e}\right)^{-1} \mathbf{o}_{n}^{e}
$$

with the Jacobian $\mathbf{J}^{e}$ expressed by

$$
\mathbf{J}^{e}=\left[\begin{array}{cc}
\frac{\partial x}{\partial \xi^{e}} & \frac{\partial y}{\partial \xi^{e}} \\
\frac{\partial x}{\partial \eta^{e}} & \frac{\partial y}{\partial \eta^{e}}
\end{array}\right]
$$

The Jacobian can be elaborated further from the definition of the displacement field $\mathbf{u}^{e}$ within an element $e$ :

$$
\begin{aligned}
\mathbf{u}^{e} & =\left[u_{x}^{e}, u_{y}^{e}\right]^{T} \\
& =\left[\sum_{n=1}^{4} N_{n}\left(\xi^{e}, \eta^{e}\right) x_{n}, \sum_{n=1}^{4} N_{n}\left(\xi^{e}, \eta^{e}\right) y_{n}\right]^{\mathrm{T}},
\end{aligned}
$$

where $x_{n}$ and $y_{n}$ are the coordinates of the element nodes. Writing the displacement at an arbitrary point within an element $e$ as the difference between its actual coordinate and its initial coordinate, $u_{x}^{e}=x-x_{0}$ and $u_{y}^{e}=y-y_{0}$, together with Eq. 2.27 the Jacobian, Eq. (2.26), becomes

$$
\mathbf{J}^{e}=\left[\begin{array}{cc}
\sum_{n=1}^{4} \frac{\partial N_{n}}{\partial \xi^{e}} x_{n} & \sum_{n=1}^{4} \frac{\partial N_{n}}{\partial \xi^{e}} y_{n} \\
\sum_{n=1}^{4} \frac{\partial N_{n}}{\partial \eta^{e}} x_{n} & \sum_{n=1}^{4} \frac{\partial N_{n}}{\partial \eta^{e}} y_{n}
\end{array}\right],
$$

by which $\mathbf{B}^{e}$ in Eq. 2.21) can be expressed in terms of $\xi^{e}$ and $\eta^{e}$ using Eqs. (2.22), 2.24, 2.25 and Eq. 2.28. 


\subsection{Structural analysis}

For a 2D plane-stress state, the constitutive matrix $\mathbf{D}^{e}$ within an element reads

$$
\mathbf{D}^{e}=\frac{E^{e}}{1-\nu^{2}}\left[\begin{array}{ccc}
1 & \nu & 0 \\
\nu & 1 & 0 \\
0 & 0 & \frac{1-\nu}{2}
\end{array}\right]
$$

where $\nu$ is Poisson's ratio (which, for simplicity, is taken as uniform across the whole design domain), and $E^{e}$ is the Young's modulus of an element, which, in accordance with the SIMP approach for topology optimization, may be formulated as [43, 66, 67. 93

$$
E^{e}=\left(\rho^{e}\right)^{p} E_{0}
$$

with $\rho^{e}$ the relative density within an element, $p$ a penalization factor on the bulk stiffness (a typical value being 3 , see [66]), and $E_{0}$ the initial Young's modulus of the material.

With Eqs. 2.21 to 2.30, Eq. 2.20 can be used to calculate the stiffness matrix $\mathbf{k}^{e}$ for each element:

$$
\mathbf{k}^{e}=\left(\rho^{e}\right)^{p} \mathbf{k}_{0},
$$

with $\mathbf{k}_{0}$ representing the initial element stiffness matrix, computed from Eq. 2.20 , but with the constitutive matrix $\mathbf{D}^{e}$ replaced by

$$
\mathbf{D}_{0}=\frac{E_{0}}{1-\nu^{2}}\left[\begin{array}{ccc}
1 & \nu & 0 \\
\nu & 1 & 0 \\
0 & 0 & \frac{1-\nu}{2}
\end{array}\right] \text {. }
$$

Finally, the global stiffness matrix $\mathbf{K}$ can be constructed by assembling the contributions of the element stiffness matrices $\mathbf{k}^{e}$ :

$$
\mathbf{K}=\sum_{e=1}^{N} \mathbf{k}^{e}
$$

where $N$ is the total number of elements, and the sum operator represents the typical element assembly procedure used in the finite element method. Denoting $\mathbf{K}_{b c}$ as the global stiffness matrix obtained after incorporating the boundary conditions, the nodal displacements result from inverting Eq. 2.2, i.e., 


$$
\mathbf{u}=\mathbf{K}_{b c}^{-1} \mathbf{f}
$$

This result can be used to compute the structural compliance as

$$
c=\mathbf{u}^{\mathrm{T}} \mathbf{K}_{b c} \mathbf{u} .
$$

In addition, the volume of each element $e$ follows from

$$
V^{e}=\sum_{i^{*}=1}^{t^{*}} \sum_{j^{*}=1}^{t^{*}} w_{i^{*}} w_{j^{*}}\left|\boldsymbol{J}^{e}\right|_{i^{*}, j^{*}},
$$

which, in accordance with Eq. (2.1), can be employed to calculate the total material volume required for topology optimization

$$
V=\sum_{e=1}^{N} \rho^{e} V^{e}
$$

as well as the volume of the initial design domain

$$
V_{0}=\sum_{e=1}^{N} V^{e} .
$$

\subsection{Sensitivity analysis and update of design vari- ables}

In order to establish a gradient-based optimization framework, the sensitivity of the objective (i.e., the structural compliance) with respect to the shape design variables (net of control points) and the topology optimization variables (element relative densities) will be derived in this section. Subsequently, the update procedure of the design variables is discussed.

\subsubsection{Shape sensitivity analysis}

As already explained, the meshing of the design domain is performed by projecting the nodal coordinates $\left(\xi_{n}, \eta_{n}\right)$ of an auxiliary mesh in the parametric domain to the nodal coordinates $\left(x_{n}, y_{n}\right)$ of the actual finite elements in the physical domain. Using the NURBS surface definition given by Eq. (2.18), with the dependencies on the nodal coordinates of the auxiliary mesh indicated, this projection reads 


$$
\left(x_{n}, y_{n}\right)=\left(\frac{\mathbf{S}^{\mathrm{T}}\left(\xi_{n}\right) \mathbf{X} \mathbf{T}\left(\eta_{n}\right)}{\mathbf{S}^{\mathrm{T}}\left(\xi_{n}\right) \mathbf{W} \mathbf{T}\left(\eta_{n}\right)}, \frac{\mathbf{S}^{\mathrm{T}}\left(\xi_{n}\right) \mathbf{Y} \mathbf{T}\left(\eta_{n}\right)}{\mathbf{S}^{\mathrm{T}}\left(\xi_{n}\right) \mathbf{W T}\left(\eta_{n}\right)}\right) .
$$

Inserting Eq. 2.39 into Eq. 2.28), the Jacobian $\mathbf{J}^{e}$ at element level can be expressed in terms of NURBS surface characteristics:

$$
\mathbf{J}^{e}=\left[\begin{array}{cc}
\sum_{n=1}^{4} \frac{\partial N_{n}}{\partial \xi^{e}} \frac{\mathbf{S}^{\mathrm{T}} \mathbf{X} \mathbf{T}}{\mathbf{S}^{\mathrm{T}} \mathbf{W T}} & \sum_{n=1}^{4} \frac{\partial N_{n}}{\partial \xi^{e}} \frac{\mathbf{S}^{\mathrm{T}} \mathbf{Y T}}{\mathbf{S}^{\mathrm{T}} \mathbf{W T}} \\
\sum_{n=1}^{4} \frac{\partial N_{n}}{\partial \eta^{e}} \frac{\mathbf{S}^{\mathrm{T}} \mathbf{X T}}{\mathbf{S}^{\mathrm{T}} \mathbf{W T}} & \sum_{n=1}^{4} \frac{\partial N_{n}}{\partial \eta^{e}} \frac{\mathbf{S}^{\mathrm{T}} \mathbf{Y T}}{\mathbf{S}^{\mathrm{T}} \mathbf{W T}}
\end{array}\right],
$$

in which the dependency of the basis functions $\mathbf{S}$ and $\mathbf{T}$ on, respectively, $\xi_{n}$ and $\eta_{n}$ have been omitted for reasons of brevity. In the domain shape optimization process, a limited number of control points is typically used for varying the shape. These control points are stored in the vector of design variables a. With the use of Eq. 2.40), the derivative of the Jacobian $\mathbf{J}^{e}$ with respect to a single design variable $a_{m}$ can be expressed as

$$
\frac{\partial \mathbf{J}^{e}}{\partial a_{m}}=\left[\begin{array}{cc}
\sum_{n=1}^{4} \frac{\partial N_{n}}{\partial \xi^{e}} \frac{\mathbf{S}^{\mathrm{T}} \frac{\partial \mathbf{X}}{\partial a_{m}} \mathbf{T}}{\mathbf{S}^{\mathrm{T}} \mathbf{W} \mathbf{T}} & \sum_{n=1}^{4} \frac{\partial N_{n}}{\partial \xi^{e}} \frac{\mathbf{S}^{\mathrm{T}} \frac{\partial \mathbf{Y}}{\partial a_{m} \mathbf{T}}}{\mathbf{S}^{\mathrm{T}} \mathbf{W T}} \\
\sum_{n=1}^{4} \frac{\partial N_{n}}{\partial \eta^{e}} \frac{\mathbf{S}^{\mathrm{T}} \frac{\partial \mathbf{X}}{\partial a_{m}} \mathbf{T}}{\mathbf{S}^{\mathrm{T}} \mathbf{W} \mathbf{T}} & \sum_{n=1}^{4} \frac{\partial N_{n}}{\partial \eta^{e}} \frac{\mathbf{S}^{\mathrm{T}} \frac{\partial \mathbf{Y}}{\partial a_{m}} \mathbf{T}}{\mathbf{S}^{\mathrm{T}} \mathbf{W} \mathbf{T}}
\end{array}\right] .
$$

Furthermore, from Eq. 2.40 the determinant of the Jacobian matrix becomes

$$
\begin{aligned}
\left|\mathbf{J}^{e}\right|= & \left(\sum_{n=1}^{4} \frac{\partial N_{n}}{\partial \xi^{e}} \frac{\mathbf{S}^{\mathrm{T}} \mathbf{X} \mathbf{T}}{\mathbf{S}^{\mathrm{T}} \mathbf{W} \mathbf{T}}\right)\left(\sum_{n=1}^{4} \frac{\partial N_{n}}{\partial \eta^{e}} \frac{\mathbf{S}^{\mathrm{T}} \mathbf{Y} \mathbf{T}}{\mathbf{S}^{\mathrm{T}} \mathbf{W} \mathbf{T}}\right) \\
& -\left(\sum_{n=1}^{4} \frac{\partial N_{n}}{\partial \xi^{e}} \frac{\mathbf{S}^{\mathrm{T}} \mathbf{Y} \mathbf{T}}{\mathbf{S}^{\mathrm{T}} \mathbf{W} \mathbf{T}}\right)\left(\sum_{n=1}^{4} \frac{\partial N_{n}}{\partial \eta^{e}} \frac{\mathbf{S}^{\mathrm{T}} \mathbf{X} \mathbf{T}}{\mathbf{S}^{\mathrm{T}} \mathbf{W} \mathbf{T}}\right) .
\end{aligned}
$$

With this expression, the partial derivative of the determinant of the Jacobian $\left|\mathbf{J}^{e}\right|$ with respect to design variable $a_{m}$ results from applying the chain rule: 


$$
\begin{aligned}
\frac{\partial\left|\mathbf{J}^{e}\right|}{\partial a_{m}}= & \left(\sum_{n=1}^{4} \frac{\partial N_{n}}{\partial \xi^{e}} \frac{\mathbf{S}^{\mathrm{T}} \frac{\partial \mathbf{X}}{\partial a_{m}} \mathbf{T}}{\mathbf{S}^{\mathrm{T}} \mathbf{W} \mathbf{T}}\right)\left(\sum_{n=1}^{4} \frac{\partial N_{n}}{\partial \eta^{e}} \frac{\mathbf{S}^{\mathrm{T}} \mathbf{Y} \mathbf{T}}{\mathbf{S}^{\mathrm{T}} \mathbf{W} \mathbf{T}}\right) \\
& +\left(\sum_{n=1}^{4} \frac{\partial N_{n}}{\partial \xi^{e}} \frac{\mathbf{S}^{\mathrm{T}} \mathbf{X} \mathbf{T}}{\mathbf{S}^{\mathrm{T}} \mathbf{W T}}\right)\left(\sum_{n=1}^{4} \frac{\partial N_{n}}{\partial \eta^{e}} \frac{\mathbf{S}^{\mathrm{T}} \frac{\partial \mathbf{Y}}{\partial a_{m}} \mathbf{T}}{\mathbf{S}^{\mathrm{T}} \mathbf{W} \mathbf{T}}\right) \\
& -\left(\sum_{n=1}^{4} \frac{\partial N_{n}}{\partial \xi^{e}} \frac{\mathbf{S}^{\mathrm{T}} \frac{\partial \mathbf{Y}}{\partial a_{m}} \mathbf{T}}{\mathbf{S}^{\mathrm{T}} \mathbf{W} \mathbf{T}}\right)\left(\sum_{n=1}^{4} \frac{\partial N_{n}}{\partial \eta^{e}} \frac{\mathbf{S}^{\mathrm{T}} \mathbf{X} \mathbf{T}}{\mathbf{S}^{\mathrm{T}} \mathbf{W} \mathbf{T}}\right) \\
& -\left(\sum_{n=1}^{4} \frac{\partial N_{n}}{\partial \xi^{e}} \frac{\mathbf{S}^{\mathrm{T}} \mathbf{Y} \mathbf{T}}{\mathbf{S}^{\mathrm{T}} \mathbf{W} \mathbf{T}}\right)\left(\sum_{n=1}^{4} \frac{\partial N_{n}}{\partial \eta^{e}} \frac{\mathbf{S}^{\mathrm{T}} \frac{\partial \mathbf{X}}{\partial a_{m}} \mathbf{T}}{\mathbf{S}^{\mathrm{T}} \mathbf{W} \mathbf{T}}\right)
\end{aligned}
$$

Combining Eqs. 2.21, 2.22) and 2.242 , the partial derivative of the element straindisplacement matrix with respect to the design variable $a_{m}$ can be derived as

$$
\frac{\partial \mathbf{B}^{e}}{\partial a_{m}}=\left[\begin{array}{llll}
\frac{\partial \mathbf{B}_{1}^{e}}{\partial a_{m}} & \frac{\partial \mathbf{B}_{2}^{e}}{\partial a_{m}} & \frac{\partial \mathbf{B}_{3}^{e}}{\partial a_{m}} & \frac{\partial \mathbf{B}_{4}^{e}}{\partial a_{m}}
\end{array}\right]
$$

where

$$
\frac{\partial \mathbf{B}_{n}^{e}}{\partial a_{m}}=\left[\begin{array}{cc}
\frac{\partial v_{1, n}^{e}}{\partial a_{m}} & 0 \\
0 & \frac{\partial v_{2, n}^{e}}{\partial a_{m}} \\
\frac{\partial v_{2, n}^{e}}{\partial a_{m}} & \frac{\partial v_{1, n}^{e}}{\partial a_{m}}
\end{array}\right] \quad \text { with } \quad n \in\{1,2,3,4\}
$$

in which, with the use of Eq. 2.25, it follows that

$$
\begin{aligned}
\frac{\partial \mathbf{v}_{n}^{e}}{\partial a_{m}}=\frac{\partial\left(\mathbf{J}^{e}\right)^{-1}}{\partial a_{m}} \mathbf{o}_{n}^{e} & =-\left(\mathbf{J}^{e}\right)^{-1} \frac{\partial \mathbf{J}^{e}}{\partial a_{m}}\left(\mathbf{J}^{e}\right)^{-1} \mathbf{o}_{n}^{e} \\
& =-\left(\mathbf{J}^{e}\right)^{-1} \frac{\partial \mathbf{J}^{e}}{\partial a_{m}} \mathbf{v}_{n}^{e},
\end{aligned}
$$

where $\partial \mathbf{J}^{e} / \partial a_{m}$ is given by Eq. 2.41). Furthermore, combining Eq. 2.20) with Eqs. 2.31 and 2.32, the derivative of $\mathbf{k}_{0}$ with respect to design variable $a_{m}$ can be expressed as

$$
\frac{\partial \mathbf{k}_{0}}{\partial a_{m}}=\sum_{i^{*}=1}^{t^{*}} \sum_{j^{*}=1}^{t^{*}} w_{i^{*}} w_{j^{*}}\left(\begin{array}{c}
\frac{\partial\left(\mathbf{B}^{e}\right)^{\mathrm{T}}}{\partial a_{m}} \mathbf{D}_{0} \mathbf{B}^{e}\left|\mathbf{J}^{e}\right| \\
+\left(\mathbf{B}^{e}\right)^{\mathrm{T}} \mathbf{D}_{0} \frac{\partial \mathbf{B}^{e}}{\partial a_{m}}\left|\mathbf{J}^{e}\right| \\
+\left(\mathbf{B}^{e}\right)^{\mathrm{T}} \mathbf{D}_{0} \mathbf{B}^{e} \frac{\partial\left|\mathbf{J}^{e}\right|}{\partial a_{m}}
\end{array}\right)_{i^{*}, j^{*}}
$$


in which $\partial \mathbf{B}^{e} / \partial a_{m}$ and $\partial\left|\mathbf{J}^{e}\right| / \partial a_{m}$ are provided by Eqs. 2.45) and (2.43), respectively. Subsequently, from Eq. 2.31 the partial derivative of the element stiffness matrix $\mathbf{k}^{e}$ with respect to the design variable $a_{m}$ is calculated via

$$
\frac{\partial \mathbf{k}^{e}}{\partial a_{m}}=\left(\rho^{e}\right)^{p} \frac{\partial \mathbf{k}_{0}}{\partial a_{m}}
$$

with $\partial \mathbf{k}_{0} / \partial a_{m}$ thus given by Eq. 2.47). Eq. 2.48 is substituted into the partial derivative of the total stiffness matrix $\mathbf{K}$ with respect to the design variable $a_{m}$, which via Eq. 2.33 becomes:

$$
\frac{\partial \mathbf{K}}{\partial a_{m}}=\sum_{e=1}^{N} \frac{\partial \mathbf{k}^{e}}{\partial a_{m}} .
$$

After taking into account the boundary conditions, $\mathbf{K} \rightarrow \mathbf{K}_{b c}$, Eq. 2.49 is used to construct the partial derivative of the displacement $\mathbf{u}$ with respect to design variable $a_{m}$, which, with Eq. 2.34, leads to

$$
\frac{\partial \mathbf{u}}{\partial a_{m}}=\mathbf{K}_{b c}^{-1}\left(\frac{\partial \mathbf{f}}{\partial a_{m}}-\frac{\partial \mathbf{K}_{b c}}{\partial a_{m}} \mathbf{u}\right) .
$$

When assuming that the external loads $\mathbf{f}$ are independent of the design variables $a_{m}$, Eq. 2.50 reduces to

$$
\frac{\partial \mathbf{u}}{\partial a_{m}}=-\mathbf{K}_{b c}^{-1} \frac{\partial \mathbf{K}_{b c}}{\partial a_{m}} \mathbf{u}
$$

In order to perform the domain shape optimization procedure given by Eq. 2.4), from Eq. 2.35 the partial derivative of the structural compliance $c$ with respect to design variable $a_{m}$ needs to be calculated, which, after substituting Eq. [2.51), results in

$$
\frac{\partial c}{\partial a_{m}}=-\mathbf{u}^{\mathrm{T}} \frac{\partial \mathbf{K}_{b c}}{\partial a_{m}} \mathbf{u}
$$

The partial derivative of the material volume $V$ with respect to design variable $a_{m}$ can be formulated based on Eqs. 2.36) and (2.37), leading to

$$
\frac{\partial V}{\partial a_{m}}=\sum_{e=1}^{N} \rho^{e} \frac{\partial V^{e}}{\partial a_{m}}
$$

with

$$
\frac{\partial V^{e}}{\partial a_{m}}=\sum_{i^{*}=1}^{t^{*}} \sum_{j^{*}=1}^{t^{*}} w_{i^{*}} w_{j^{*}}\left(\frac{\partial\left|\mathbf{J}^{e}\right|}{\partial a_{m}}\right)_{i^{*}, j^{*}},
$$

whereby $\partial\left|\mathbf{J}^{e}\right| / \partial a_{m}$ is given by Eq. 2.43. 


\subsubsection{Topology sensitivity analysis}

To perform the topology optimization procedure presented in Eq. (2.1), similar to Eq. (2.52) the partial derivative of the structural compliance $c$ with respect to the relative density $\rho^{e}$ is computed as

$$
\frac{\partial c}{\partial \rho^{e}}=-\mathbf{u}^{\mathrm{T}} \frac{\partial \mathbf{K}_{b c}}{\partial \rho^{e}} \mathbf{u}
$$

The partial derivative of the element stiffness matrix $\mathbf{k}^{e}$ with respect to $\rho^{e}$ can be determined via Eq. 2.31:

$$
\frac{\partial \mathbf{k}_{e}}{\partial \rho^{e}}=p\left(\rho^{e}\right)^{p-1} \mathbf{k}_{0}
$$

Hence, the dependency of the total stiffness matrix $\mathbf{K}_{b c}$ on $\rho^{e}$ enters through the individual element stiffness matrices $\mathbf{k}^{e}$. Accordingly, Eq. 2.55 is developed for a specific element $e$ using Eq. 2.56, i.e.,

$$
\frac{\partial c}{\partial \rho^{e}}=-\left(\mathbf{u}^{e}\right)^{\mathrm{T}} \frac{\partial \mathbf{k}^{e}}{\partial \rho^{e}} \mathbf{u}^{e}=-\left(\mathbf{u}^{e}\right)^{\mathrm{T}} p\left(\rho^{e}\right)^{p-1} \mathbf{k}_{0} \mathbf{u}^{e} .
$$

In order to increase the robustness of the solution procedure and to alleviate possible checkerboard patterns in the spatial distribution of the element relative densities, Eq. 2.57) is slightly modified by adopting the sensitivity filter suggested in [66], i.e.,

$$
\frac{\partial \hat{c}}{\partial \rho^{e}}=\frac{1}{\rho^{e} \sum_{f=1}^{N} \hat{H}^{f}} \sum_{f=1}^{N} \hat{H}^{f} \rho^{f} \frac{\partial c}{\partial \rho^{f}},
$$

in which $\hat{H}^{f}=\max \left(0, r_{\min }-\operatorname{dist}(e, f)\right)$, where $\operatorname{dist}(e, f)$ is the distance between the center of element $e$ and the center of element $f$, and $r_{\min }$ is the radius adopted for the sensitivity filter. The filter radius is given by $r_{\min }=r_{0} \sqrt{V^{e}}$, where $r_{0}$ is a predefined scaling parameter. Although the dependency of the filter radius $r_{\text {min }}$ on the element volume $V^{e}$ compromises the mesh-independent aspect of the sensitivity filter, it avoids the emergence of checkerboard patterns in regions where shape enlargement causes enlarged elements to lose their filtering capacity in the case the filter radius is kept fixed. It is noted that in 94] an alternative version of the sensitivity filter is presented, which also accounts for varying element sizes. Finally, from Eq. 2.37 the partial derivative of the material volume $V$ with respect to the relative density $\rho^{e}$ is calculated as

$$
\frac{\partial V}{\partial \rho^{e}}=V^{e}
$$




\subsubsection{Update of design variables}

The domain shape optimization procedure formulated by Eq. (2.4) is carried out by applying a Sequential Quadratic Programming (SQP) method, using the fmincon solver in MATLAB (Version R2016b), see [95] for more details. The element densities within the topology optimization procedure are updated using the so-called Optimality Criteria (OC) method described in 66. The topology optimization algorithm used in the present work is based on the formulation presented in 66. In this algorithm, however, all finite element volumes are taken as constant, which in the present approach is not the case, due to the combination with the shape optimization algorithm. Therefore, some minor adaptations were made to this topology optimization algorithm in order to account for the variability in element volume.

\subsection{Numerical examples}

\subsubsection{Cantilever beam problem}

A popular benchmark problem for evaluating optimization methods is the cantilever beam problem 66, 67, 96, 97. The coupled gradient-based topology and domain shape optimization approach will be applied to this benchmark problem, whereby the goal is to determine the geometry with a minimal structural compliance $c$, subject to appropriate geometrical constraints and a target material volume.

The initial configuration of an elastic cantilever beam with Young's modulus $E$ and Poisson's ratio $\nu$ is shown in Figure 2.3. The left side of the beam is fully clamped (zero displacements along the left edge in all directions), and a vertical load $F$ is applied at point $\mathrm{Q}$ at the right edge. The beam has a fixed length $L$ and an initial height $H_{0}$, whereby during domain shape optimization the actual height $H$ of the beam may vary between $H_{\min }$ and $H_{\max }$. The material volume $V$ obtained after the optimization procedure should be $40 \%$ of that of the initial configuration, $V_{0}$, in correspondence with a material volume fraction $f_{r}=0.4$. The first problem that will be analyzed is referred to as Case 1, for which the values of the parameters introduced above are summarized in Table 2.1. Subsequently, two other problems will be studied, referred to as Case 2 and Case 3. Here, Case 2 is similar to Case 1, but the simulation now starts from the maximal beam height, $H_{0}=H_{\max }$, while in Case 3 the beam height is left unbounded by not prescribing a maximal value $H_{\max }$.

Table 2.1: Parameter values for the Case 1 cantilever beam problem.

\begin{tabular}{lllll}
\hline Parameter & $L(\mathrm{~m})$ & $H_{0}(\mathrm{~m})$ & $F(\mathrm{kN})$ & $V_{0}\left(\mathrm{~m}^{2}\right)$ \\
\hline Value & 30 & 7 & -10 & 210 \\
\hline Parameter & $H_{\min }(\mathrm{m})$ & $H_{\max }(\mathrm{m})$ & $E(\mathrm{MPa})$ & $\nu$ \\
\hline Value & 1 & 10 & $210 \times 10^{3}$ & 0.3 \\
\hline
\end{tabular}

The geometry of the cantilever beam is described by NURBS. Setting the polyno- 


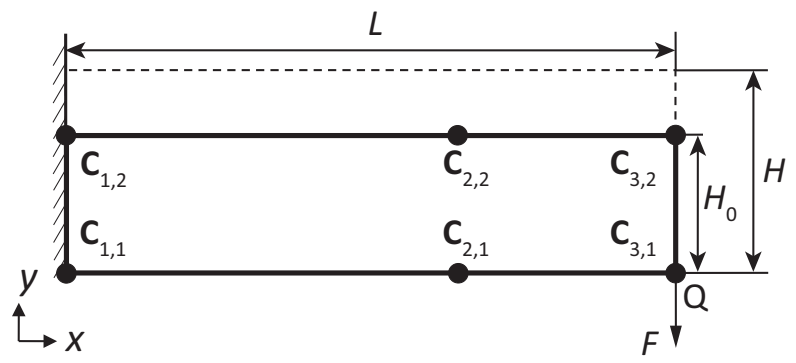

Figure 2.3: Elastic cantilever beam with the control points $\mathbf{C}_{i, j}$ indicated.

mial order of the basis functions, Eq. (2.7), as $p=2$ and $q=1$, the knot vectors for the B-spline surface, Eq. (2.6), can be defined as

$$
\begin{aligned}
& \boldsymbol{\Xi}=[0,0,0,1,1,1]^{\mathrm{T}}, \\
& \boldsymbol{\Theta}=[0,0,1,1]^{\mathrm{T}},
\end{aligned}
$$

which correspond to a total of 6 control points. Table 2.2 lists the initial coordinates and weights of these control points. For simplicity, all weights have been chosen equal to unity, by which the NURBS surface reduces to a B-spline surface, see Eq. (2.8) and Eqs. 2.10 to 2.14). The $y$-coordinates of the control points $\mathbf{C}_{1,2}, \mathbf{C}_{2,2}, \mathbf{C}_{3,2}$ govern the height, and thus the shape of the cantilever beam. As such, the vector with shape design variables $\boldsymbol{a}$ can be expressed as

$$
\boldsymbol{a}=\left[Y_{1,2}, Y_{2,2}, Y_{3,2}\right]^{\mathrm{T}} .
$$

Table 2.2: Coordinates $\mathbf{C}_{i, j}$ (in $\mathrm{m}$ ) and weights $\omega_{i, j}$ of control points.

\begin{tabular}{lllll}
\hline$i$ & $j=1$ & $j=2$ & \\
\hline 1 & $\mathbf{C}_{1,1}=(0,0)$ & $\omega_{1,1}=1$ & $\mathbf{C}_{1,2}=(0,7)$ & $\omega_{1,2}=1$ \\
\hline 2 & $\mathbf{C}_{2,1}=(20,0)$ & $\omega_{2,1}=1$ & $\mathbf{C}_{2,2}=(20,7)$ & $\omega_{2,2}=1$ \\
\hline 3 & $\mathbf{C}_{3,1}=(30,0)$ & $\omega_{3,1}=1$ & $\mathbf{C}_{3,2}=(30,7)$ & $\omega_{3,2}=1$ \\
\hline
\end{tabular}

The auxiliary mesh in the initial parametric domain $\boldsymbol{\Omega}_{0}$ is constructed by $200 \times 25$ equal-sized, rectangular parts, of which the corners via Eq. 2.5 are projected on the finite element nodes in the physical domain. Accordingly, the geometry in the physical domain is meshed with 5000 4-node plane-stress elements, equipped with quadratic shape functions, see Eq. 2.23). A mesh sensitivity study presented further in this communication will demonstrate that this FEM discretization is sufficiently fine for obtaining accurate numerical results. For the domain shape optimization procedure, the settings of the MATLAB solver fmincon are as follows: the "Algorithm" is set to "sqp" (sequential quadratic programming), the "GradObj" and "GradConstr" are set 
to "on", which means that the sensitivities of the objective function and constraints used in the gradient-based domain shape optimization procedure are provided by the user. The remaining options are default. The domain shape optimization procedure is considered to be finished if the structural compliance meets the condition: $\mid c^{h+1}-$ $c^{h} \mid \leq 1 \mathrm{e}-6$, with $h$ the iteration number, or if the shape design variables satisfy the criterion: $\max \left(\left|\mathbf{a}^{h+1}-\mathbf{a}^{h}\right|\right) \leq 1 \mathrm{e}-6$. For the topology optimization, the scaling parameter $r_{0}$ characterizing the filter radius $r_{\text {min }}$ used in Eq. 2.58 is set equal to $r_{0}=1.5$. Furthermore, the move limit appearing in the OC method 66 is set to $\phi=0.4$. The topology optimization procedure is finished if the structural compliance satisfies the requirement: $\left|c^{g+1}-c^{g}\right| \leq 1 \mathrm{e}-6$, with $g$ the iteration number. Finally, the stop criterion used in the outer loop in Figure 2.1 is: $\left|c^{k+1}-c^{k}\right| \leq 1 \mathrm{e}-5$, where $k$ is the iteration number of the outer loop.

The above value of $1 \mathrm{e}-6$ chosen for the stop criteria of the shape and topology optimization inner subloops was selected based on an separate variation study, in which for the STO and the TSO update schemes described in Section 2.2 the value of these stop criteria was systematically varied in the Case 1 and Case 3 problems introduced above. The variation study clearly demonstrated that the value found for the optimized compliance $c$ increases monotonically with a growing value of the stop criteria. In specific, increasing the value of the stop criteria from 1e-6 to $1 \mathrm{e}-5$ typically results in a percentual increase of the optimized compliance between $0.03 \%$ and $0.44 \%$, while increasing the value from $1 \mathrm{e}-6$ to $1 \mathrm{e}-3$ increases the optimized compliance between $1.2 \%$ and $8.2 \%$. Based on this result, it was concluded that a value of $1 \mathrm{e}-6$ of the stop criteria for the shape and topology inner subloops results in solutions with sufficient numerical accuracy.

First the coupled topology and domain shape optimization with a TSO sequence is analyzed for Case 1. The optimization starts with a topology optimization step of the initial design domain with a fixed shape, as shown in Figure 2.4. Subsequently, a domain shape optimization step is performed whereby the finite element densities found at the end of the last topology optimization step are used as initial values, see Figure 2.5. Note that the height of the clamped support at the left side of the cantilever beam is allowed to vary during the process of domain shape optimization. According to the solution strategy illustrated in Figure 2.1, the alternating topology and shape optimization steps will continue until the stop criterion of the outer loop is satisfied. Figure 2.6 presents the value $c$ of the objective function and the values of the shape design variables, Eq. 2.61, as a function of the outer loop iterations $k$, while Figure 2.7 shows the shapes and topologies of the cantilever beam at specific iterations $k$ of the outer loop. It can be observed that during the iterative process the shape changes from convex to concave, and that the topological layout of the interior "trusses" changes significantly as well. During the optimization procedure the shape clearly has a strong influence on the topology and vice versa; hence, the optimized structural layout is a trade-off between the density distribution and the shape.

In order to investigate whether it makes a difference if the optimization procedure starts with a shape or a topology optimization step, Case 1 is also performed using an STO sequence. Figure 2.8 presents the initial, domain shape optimization step, whereby the relative densities are taken in accordance with a homogeneous distribu- 


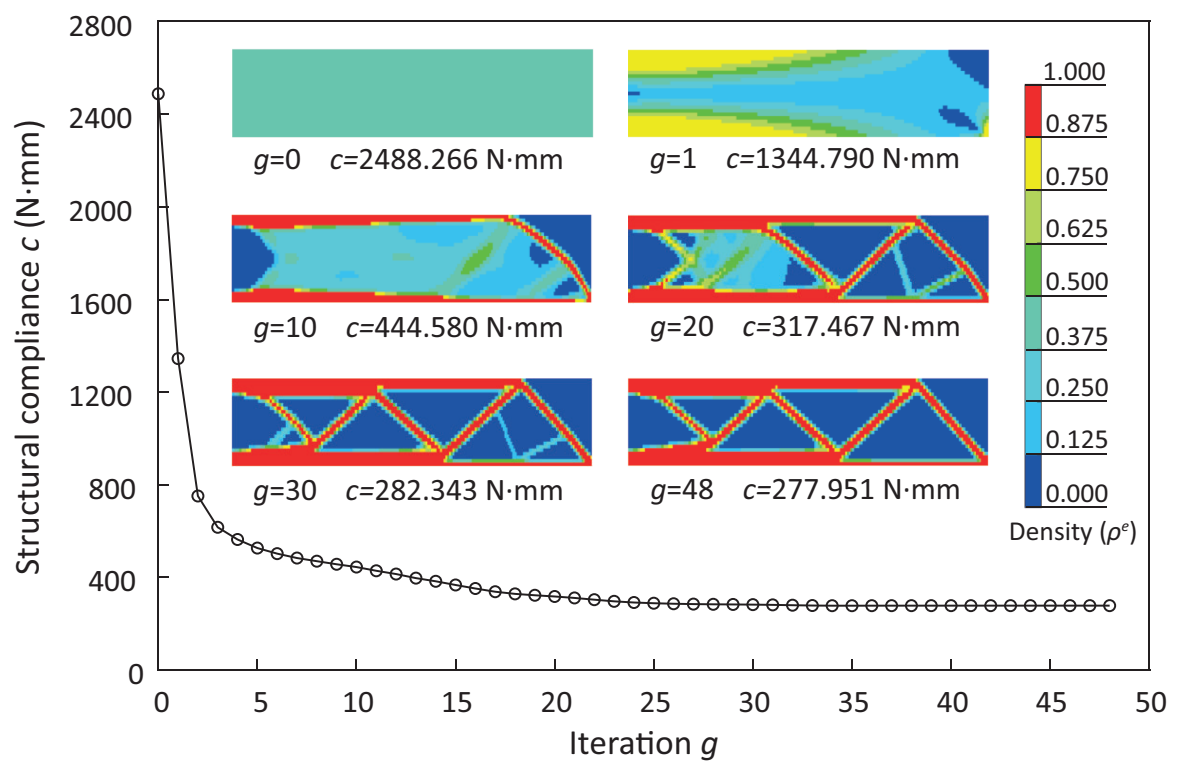

Figure 2.4: Convergence behavior of the structural compliance $c$ during the first step of Case 1: topology optimization (TSO sequence). The inset shows beam configurations at various iterations $g$.

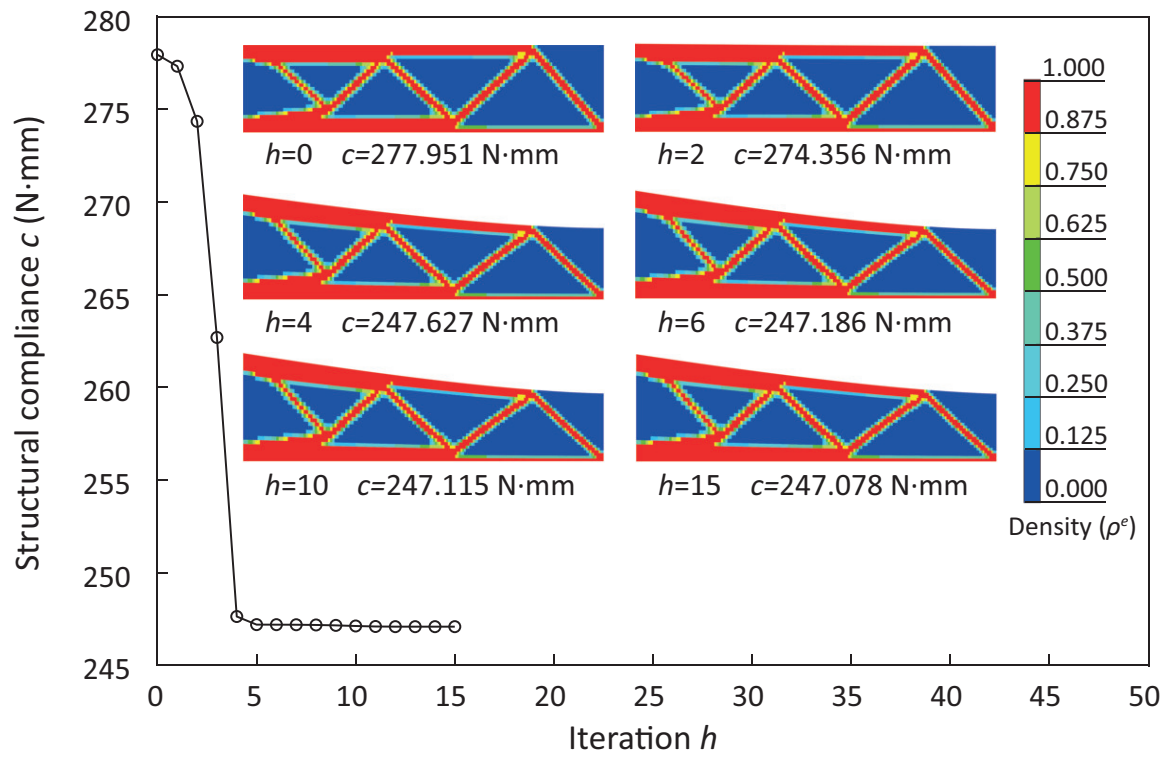

Figure 2.5: Convergence behavior of the structural compliance $c$ during the second step of Case 1: domain shape optimization (TSO sequence). The inset shows beam configurations at various iterations $h$. 


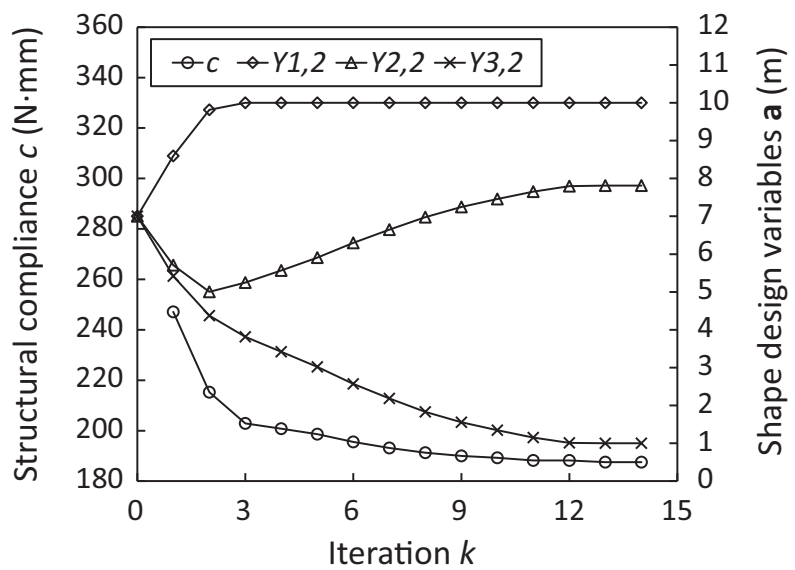

Figure 2.6: Convergence behavior of the structural compliance $c$ and the shape design variables $\boldsymbol{a}=\left[Y_{1,2}, Y_{2,2}, Y_{3,2}\right]^{\mathrm{T}}$ for Case 1 (TSO sequence).

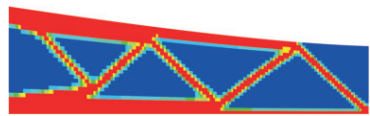

$k=1 \quad c=247.078 \mathrm{~N} \cdot \mathrm{mm}$

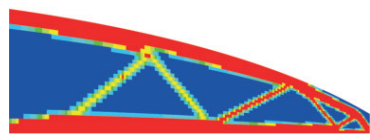

$k=9 \quad c=190.040 \mathrm{~N} \cdot \mathrm{mm}$

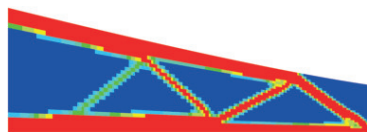

$k=3 \quad c=202.927 \mathrm{~N} \cdot \mathrm{mm}$

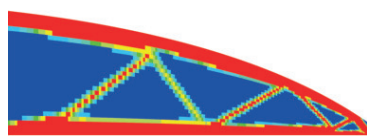

$k=12 \quad c=188.170 \mathrm{~N} \cdot \mathrm{mm}$

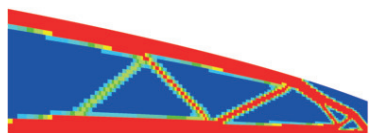

$k=6 \quad c=195.569 \mathrm{~N} \cdot \mathrm{mm}$

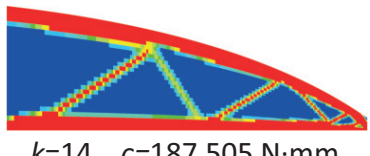

$k=14 \quad c=187.505 \mathrm{~N} \cdot \mathrm{mm}$

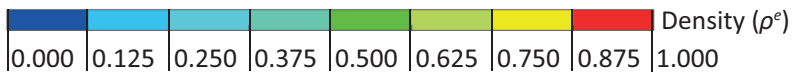

Figure 2.7: Beam configurations for Case 1 (TSO sequence) at various iterations $k$.

tion. Using the shape obtained at the end of the first step, a topology optimization step is carried out in turn, see Figure 2.9. The results obtained after successive, combined shape and topology optimization steps are shown in Figures 2.10 and 2.11 illustrating, respectively, the convergence behavior of the structural compliance $c$ and the shape design variables $\boldsymbol{a}$, and sketching the corresponding structural configurations at various iterations $k$ of the outer loop. Comparing the results for the present STO sequence with those for the TSO sequence depicted in Figures 2.6 and 2.7 shows that the TSO and STO sequences lead to a similar final configuration, but that the TSO sequence requires more iterations $(k=14)$ to reach this configuration than the STO sequence $(k=10)$. For both the TSO and STO sequence the number of the inner iterations $g$ (topology) and $h$ (domain shape) decrease with an increase of the outer iterations $k$. Although the rate of decrease generally is problem-dependent, the number of inner iterations at the final outer iteration before convergence turns out to 


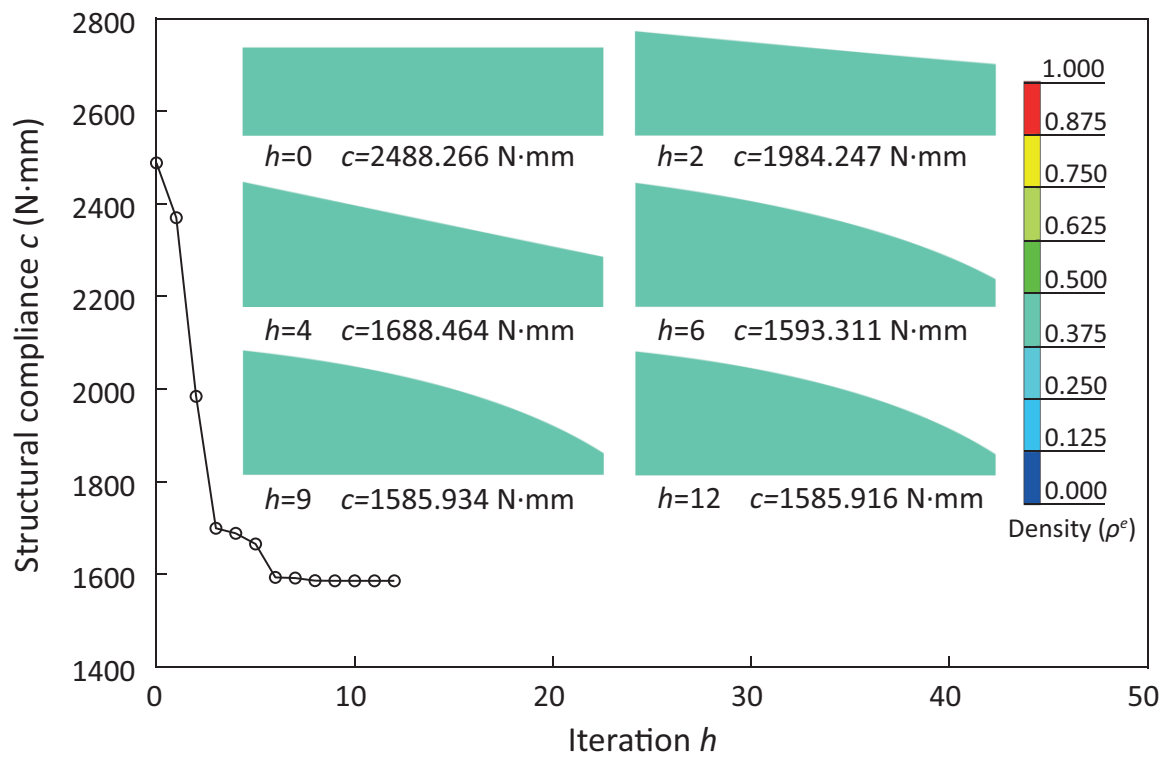

Figure 2.8: Convergence behavior of the structural compliance $c$ during the first step of Case 1: domain shape optimization (STO sequence). The inset shows beam configurations at various iterations $h$.

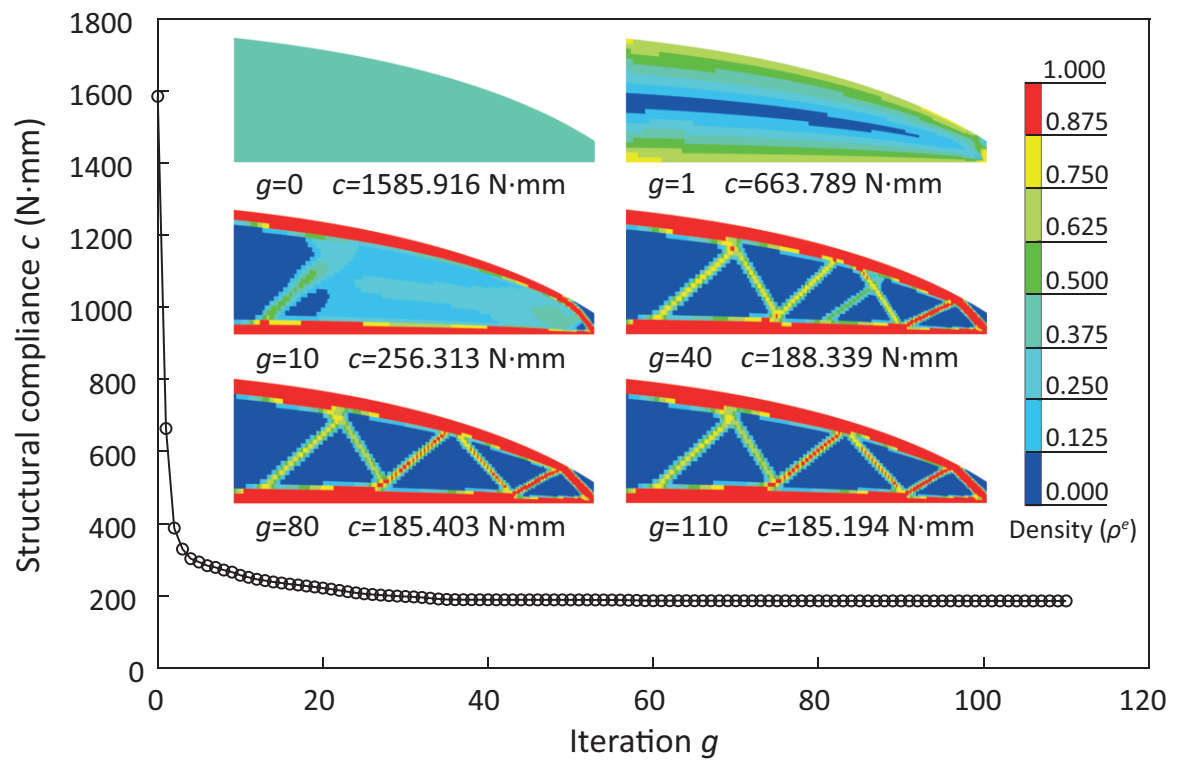

Figure 2.9: Convergence behavior of the structural compliance $c$ during the second step of Case 1: topology optimization (STO sequence). The inset shows beam configurations at various iterations $g$. 
be fairly constant, with a typical value of 2 to 3 . The differences in the final configurations of the TSO and STO sequences are reflected by the final values of the structural compliance $c$, i.e., $c=187.505 \mathrm{Nmm}$ (TSO, see Figure 2.7) versus $c=183.313 \mathrm{Nmm}$ (STO, see Figure 2.11), as well as by the final values of the shape design variables, i.e., $\boldsymbol{a}=[10.0,7.8,1.0]^{T}(\mathrm{TSO})$ versus $\boldsymbol{a}=[10.0,8.6,1.0]^{T}$ (STO, measured in $\mathrm{m}$ ), see Figures 2.6 and 2.10, respectively. The appearance of different local minima under different algorithmic schemes and search paths is well-known within the structural optimization community, see, for example, 98. The number of local minima in structural optimization problems typically is large, and is characterized by the "landscape" created by the objective function(s), the design variables and constraints, the characteristics of the boundary value problem and the FEM discretization.

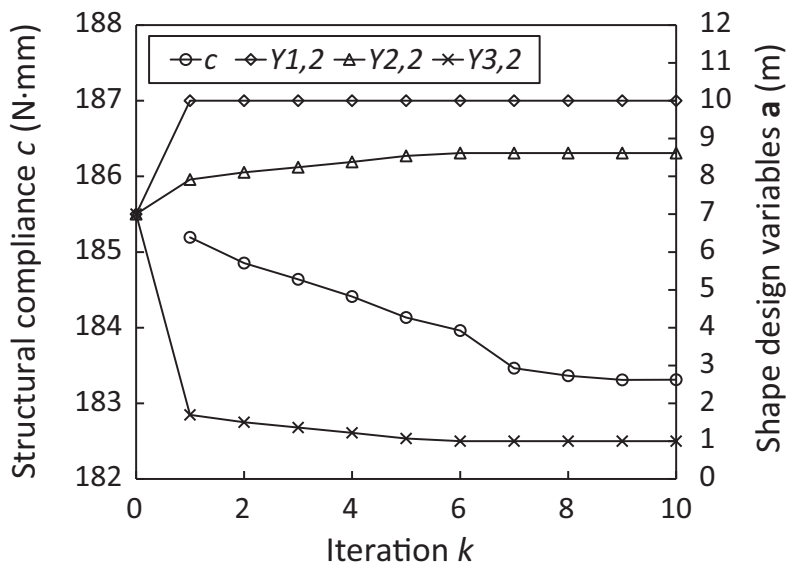

Figure 2.10: Convergence behavior of the structural compliance $c$ and the shape design variables $\boldsymbol{a}=\left[Y_{1,2}, Y_{2,2}, Y_{3,2}\right]^{\mathrm{T}}$ for Case 1 (STO sequence).
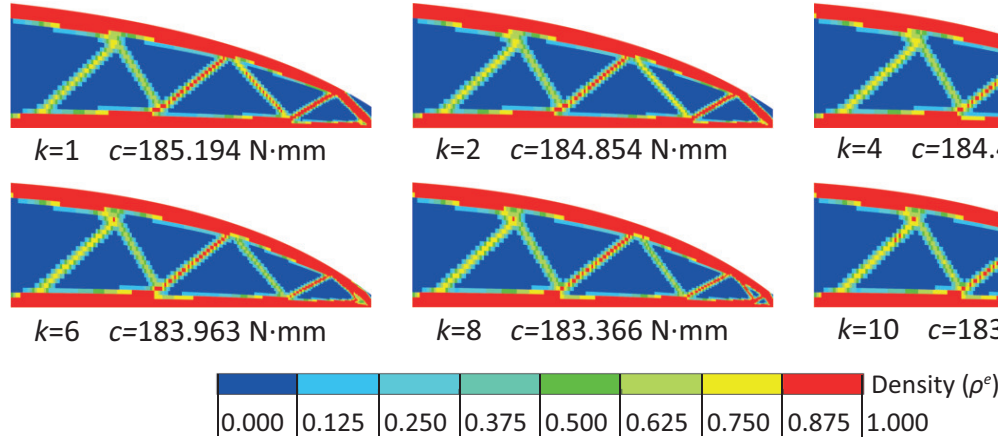

Figure 2.11: Beam configurations for Case 1 (STO sequence) at various iterations $k$.

Since in the present coupled topology and domain shape optimization method the 
finite element mesh is chosen to morph together with design domain, it is necessary to analyze the effect of the FEM discretization on the optimization result in more detail. Accordingly, a comparison study has been performed considering four different initial FEM meshes for Case 1 (STO sequence), which are characterized by uniformly discretizing the parametric domain $\boldsymbol{\Omega}_{0}$ into (a) $160 \times 20$, (b) $180 \times 20$, (c) $120 \times 40$ and (d) $200 \times 25$ elements. Note that the effect of the number of elements on the computational result is analyzed here by choosing meshes (a) and (b) to be 30 to $40 \%$ coarser than meshes (c) and (d). Figure 2.12 depicts the optimized configurations computed for the different initial meshes, together with the final FEM discretizations (whereby specific details can be distinguished by zooming in on the figures). Clearly, the various FEM meshes lead to similar layouts. In addition, for the relatively fine mesh $(d)$ the final structural compliance $c$ is only $1 \%$ higher than the value obtained for the other fine mesh (c), from which it is concluded that the difference in the initial width-to-height element aspect ratio for the two meshes (i.e., about 3:2 for mesh (c) versus 1:2 for mesh $(d)$ ) only marginally influences the final numerical result. Note, however, that the significant reduction of the structural height at the right beam end resulting from the domain shape optimization procedure causes the element width-toheight aspect ratio of mesh (c) locally to become nearly two times larger - and thus less optimized - than that of mesh $(d)$. For the relatively coarse meshes $(a)$ and $(b)$ the final structural compliance respectively is only $7 \%$ and $5 \%$ higher than for the fine mesh (c). Additionally, the number of outer iterations $k$ is the lowest for the relatively coarse mesh (a) (i.e., $k=7$ ) and the highest for the relatively fine mesh (d) (i.e., $k=10$ ). From the differences in the optimization results for the relatively coarse and fine meshes, and the minor sensitivity to the element aspect ratio used in the discretization of the two fine meshes, it may be concluded that the fine mesh (d) adopted in the forthcoming comparison study of Cases 1, 2 and 3 will provide adequate numerical results.

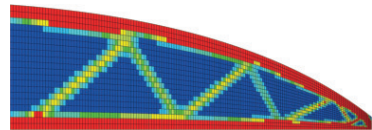

(a) $160 \times 20=3200$ $k=7 \quad c=193.497 \mathrm{~N} \cdot \mathrm{mm}$

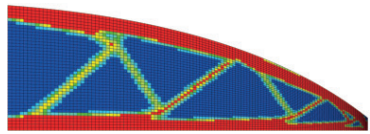

(c) $120 \times 40=4800$ $k=8 \quad c=181.197 \mathrm{~N} \cdot \mathrm{mm}$

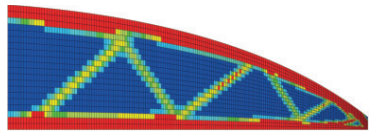

(b) $180 \times 20=3600$ $k=8 \quad c=189.813 \mathrm{~N} \cdot \mathrm{mm}$

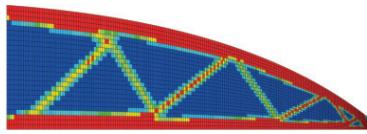

(d) $200 \times 25=5000$ $k=10 \quad c=183.313 \mathrm{~N} \cdot \mathrm{mm}$

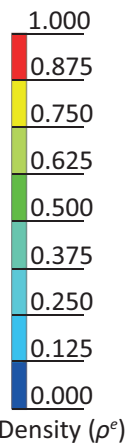

Figure 2.12: Final configuration for Case 1 (STO sequence) using different initial FEM discretizations. (a) $160 \times 20=3200$ elements, (b) $180 \times 20=3600$ elements, (c) $120 \times 40=4800$ elements, and (d) $200 \times 25=5000$ elements.

In the comparison study of the three cases, for Case 2 the height of the initial 
domain is set equal to the maximal value, $H_{0}=H_{\max }=10 \mathrm{~m}$. The volume (per unit depth) of the initial structure $\left(V_{0}=300 \mathrm{~m}^{2}\right)$ is larger than for Case $1\left(V_{0}=210\right.$ $\mathrm{m}^{2}$ ), which is compensated for by scaling down the required volume fraction $f_{r}$ in accordance with the ratio of the volumes, such that an adequate comparison between the two cases can be made, i.e., $f_{r}=(210 / 300) \times 0.4=0.28$.

In order to clarify the mutual influence of topology and shape optimization for this case, the cantilever beam is first subjected to topology optimization only. By starting from a homogeneous density distribution, the converged density distribution obtained after $g=70$ iterations is shown in Figure 2.13. The structural compliance at convergence, $c=166.325 \mathrm{Nmm}$, turns out to be lower than for the STO optimization procedure of Case 1, $c=183.313 \mathrm{Nmm}$. In other words, the search path followed for Case 2 with a pure topology optimization procedure has led to a different local minimum for the structural compliance. The question now remains whether this result will alter when the topology optimization procedure for Case 2 is combined with domain shape optimization. The outcome of this analysis is shown in Figure 2.14, illustrating that the topologies and the corresponding values of the structural compliance $c$ computed with the TSO (Figure 2.14 a) and STO (Figure 2.14 b) sequences are virtually identical to those calculated with the pure topology optimization procedure shown in Figure 2.13. Accordingly, it may be concluded that domain shape optimization has a negligible effect on the optimization of Case 2.
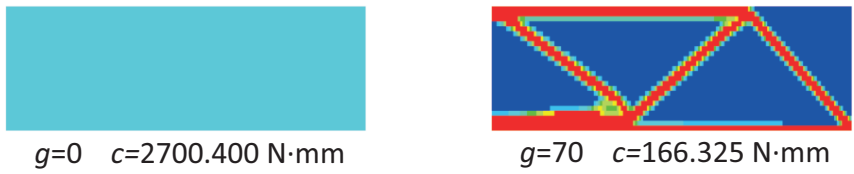

$g=70 \quad c=166.325 \mathrm{~N} \cdot \mathrm{mm}$

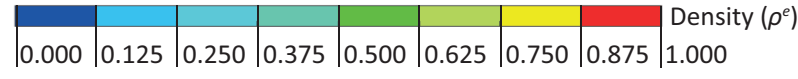

Figure 2.13: Beam configurations for Case 2 (Topology optimization only) at the onset $(g=0)$ and at the converged iteration $(g=70)$.

(a) Sequence TSO

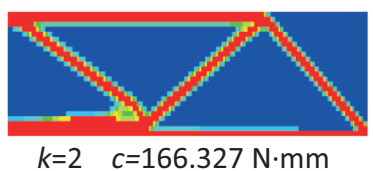
\begin{tabular}{|l|l|l|l|l|l|l|l|l|l|}
\hline 0.000 & 0.125 & 0.250 & 0.375 & 0.500 & 0.625 & 0.750 & 0.875 & $\begin{array}{l}\text { Density }\left(\rho^{e}\right) \\
1.000\end{array}$
\end{tabular} (b) Sequence STO

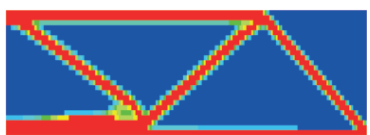

$k=2 \quad c=166.327 \mathrm{~N} \cdot \mathrm{mm}$

Figure 2.14: Beam configurations for Case 2: TSO (a) and STO (b) sequences after convergence.

The computational results for Case 3, in which the beam height is left unbounded, are shown in Figures 2.15 and 2.16 for the coupled optimization procedure with the 
TSO sequence, and in Figures 2.17 and 2.18 for the coupled optimization procedure with the STO sequence. A comparison of the topologies of the two sequences depicted in Figures 2.16 and 2.18 illustrates that these are quite similar, both leading to a triangular "truss" structure with no material present inside. The possible appearance of unfavorable element distortions in the triangular structure is avoided by locally using a relatively fine FEM mesh. As illustrated in Figure 2.19, the FEM discretization of the structure as obtained after the optimization procedure of Case 3 indeed preserves the mesh quality at sharp corners. An alternative approach that could have been applied for preventing element distortions is based on the use of so-called boundarycut elements, see [99, 100] for more details.

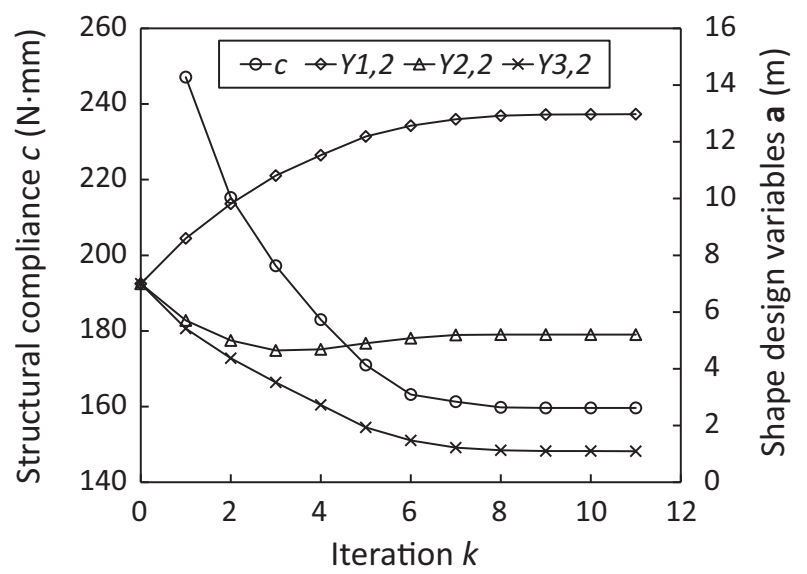

Figure 2.15: Convergence behavior of the structural compliance $c$ and the shape design variables $\boldsymbol{a}=\left[Y_{1,2}, Y_{2,2}, Y_{3,2}\right]^{\mathrm{T}}$ for Case 3 (TSO sequence).

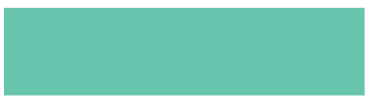

$k=0 \quad c=2488.266 \mathrm{~N} \cdot \mathrm{mm}$

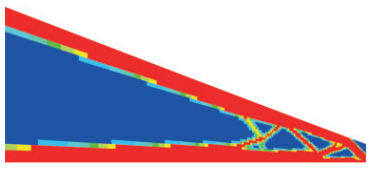

$k=6 \quad c=163.193 \mathrm{~N} \cdot \mathrm{mm}$

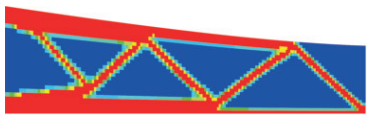

$k=1 \quad c=247.078 \mathrm{~N} \cdot \mathrm{mm}$

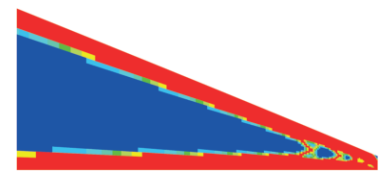

$k=9 \quad c=159.670 \mathrm{~N} \cdot \mathrm{mm}$

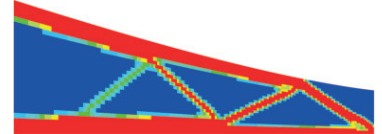

$k=3 \quad c=197.247 \mathrm{~N} \cdot \mathrm{mm}$

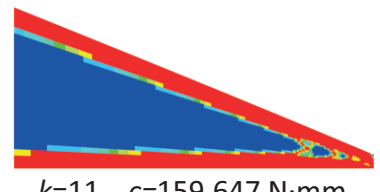

$k=11 \quad c=159.647 \mathrm{~N} \cdot \mathrm{mm}$

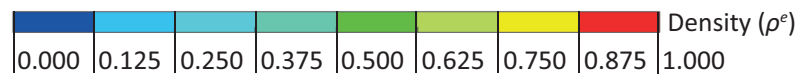

Figure 2.16: Beam configurations for Case 3 (TSO sequence) at various iterations $k$.

Observe that the final value of the structural compliance $c$ is different for the two 
update schemes, i.e., $c=159.647 \mathrm{Nmm}$ (TSO) versus $c=149.705 \mathrm{Nmm}$ (STO), and that the outer loop of the TSO sequence requires more iterations $(k=11)$ to converge than the STO sequence $(k=8)$. Furthermore, from Figures 2.15 and 2.17 it can be seen that the final values of the shape variables for the two optimization sequences also differ somewhat, i.e., $\boldsymbol{a}=[13.0,5.2,1.1]^{T}(\mathrm{TSO})$ versus $\boldsymbol{a}=[13.5,5.4,1.1]^{T}$ (STO) (measured in $\mathrm{m}$ ). Note that the final structural compliances $c$ found with the TSO and STO sequences for Case 3 are smaller than for Case 2 and Case 1. Hence, for the present cantilever beam problem the initial value and prescribed bounds of the beam height $H$ have a strong influence on the optimized shape and topology computed. Finally, in Table 2.3 the minimal compliance values are summarized together with the computational times of Cases 1, 2 and 3 for the coupled optimization procedures with TSO and STO update schemes. The STO sequence appears to be $19 \%$ to $46 \%$ faster than the TSO sequence, and results in a minimal compliance value that is up to $6 \%$ lower. Hence, it may be concluded that the performance of the coupled optimization procedure with the STO update scheme here is superior to that with the TSO update scheme.

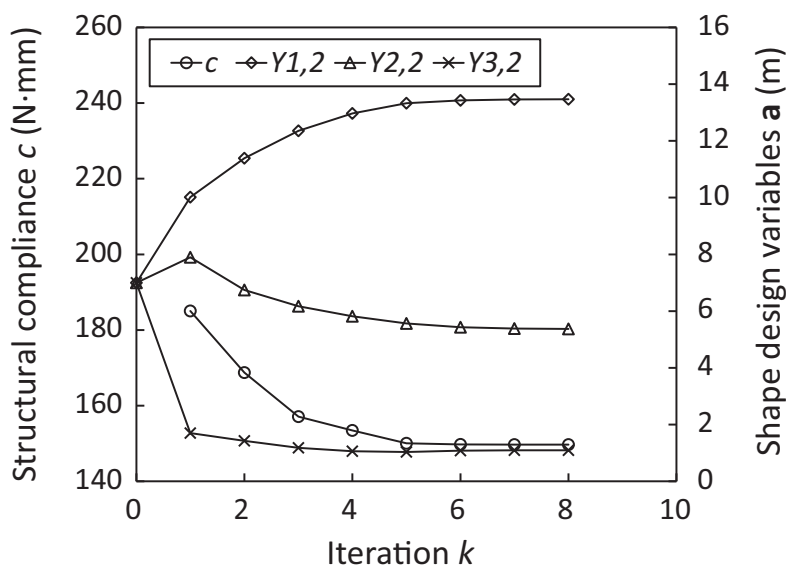

Figure 2.17: Convergence behavior of the structural compliance $c$ and the shape design variables $\boldsymbol{a}=\left[Y_{1,2}, Y_{2,2}, Y_{3,2}\right]^{\mathrm{T}}$ for Case 3 (STO sequence).

Table 2.3: Comparison of the optimized compliance value $c$ and computational time of Cases 1, 2 and 3, as calculated using the TSO and STO update schemes.

\begin{tabular}{lllll}
\hline \multirow{2}{*}{ Problem } & \multicolumn{2}{c}{ Compliance $c(\mathrm{Nmm})$} & \multicolumn{2}{c}{ Time $\left(10^{4} \mathrm{~s}\right)$} \\
& TSO & STO & TSO & STO \\
\hline Case 1 & 187.505 & 183.313 & 4.890 & 2.662 \\
\hline Case 2 & 166.327 & 166.327 & 0.262 & 0.211 \\
\hline Case 3 & 159.647 & 149.705 & 5.773 & 4.024 \\
\hline
\end{tabular}




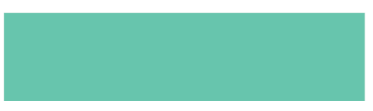

$k=0 \quad c=2488.266 \mathrm{~N} \cdot \mathrm{mm}$

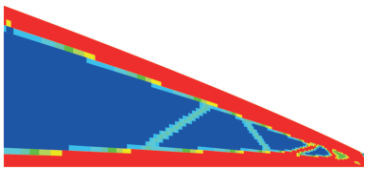

$k=4 \quad c=153.454 \mathrm{~N} \cdot \mathrm{mm}$

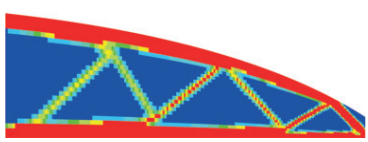

$k=1 \quad c=185.059 \mathrm{~N} \cdot \mathrm{mm}$

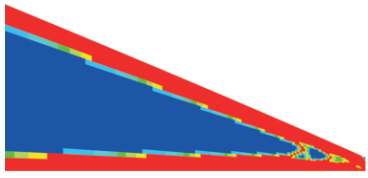

$k=6 \quad c=149.743 \mathrm{~N} \cdot \mathrm{mm}$

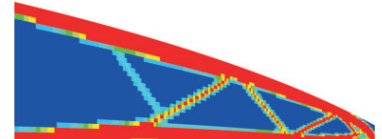

$k=2 \quad c=168.758 \mathrm{~N} \cdot \mathrm{mm}$

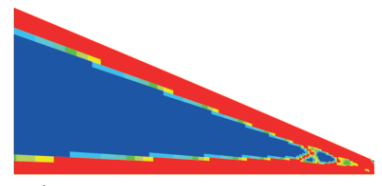

$k=8 \quad c=149.705 \mathrm{~N} \cdot \mathrm{mm}$

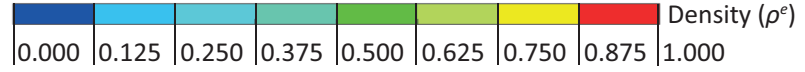

Figure 2.18: Beam configurations for Case 3 (STO sequence) at various iterations $k$.

\subsubsection{Curved L-shape beam problem}

For the cantilever beam problem analyzed above the initial design domain has a basic, rectangular shape. In this section the features of the coupled topology and domain shape optimization approach are further explored for the curved L-shape beam configuration depicted in Figure 2.20, which is characterized by a more complicated initial design domain. The L-shape beam configuration has proven to be a useful benchmark problem for the validation of topology optimization techniques, see, e.g., [76, 101-105].

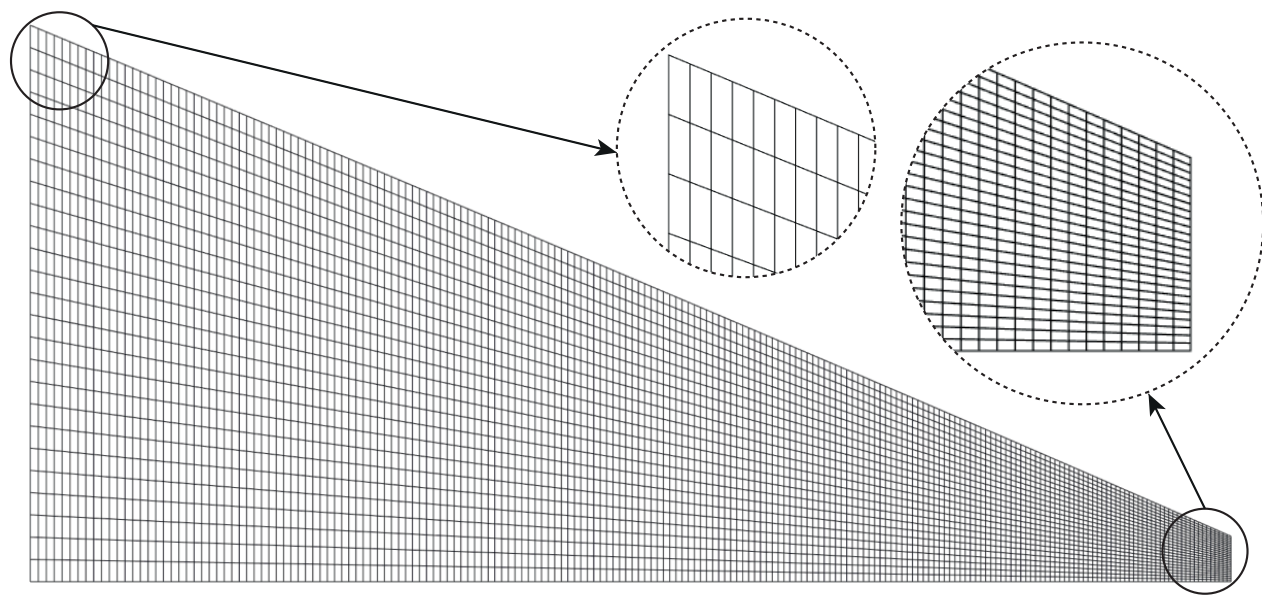

Figure 2.19: Finite element discretization of the final optimized design domain for Case 3 (STO sequence), with the mesh details at the upper left corner and right side of the beam illustrated in the insets.

As illustrated in Figure 2.20, the top side of the L-shape beam is fully clamped, and a vertical load $F=10 \mathrm{kN}$ is applied at the top corner of the free edge. The polynomial 
orders of the NURBS basis functions expressed by Eq. (2.7) are $p=3$ and $q=1$. Accordingly, the knot vectors for the B-spline surface, Eq. 2.6), are $\boldsymbol{\Xi}=[0,0,0,0,1,1,1,1]^{\mathrm{T}}$ and $\boldsymbol{\Theta}=[0,0,1,1]^{\mathrm{T}}$. The initial coordinates and weights of the 8 control points illustrated in Figure 2.20 are listed in Table 2.4. The shape design variables $\boldsymbol{a}$ are selected as $\boldsymbol{a}=\left[X_{1,1}, X_{2,1}, Y_{2,1}, X_{3,1}, Y_{3,1}\right]^{\mathrm{T}}$, with their upper and lower bounds given by $[-5,-5,-20,6,-20]^{\mathrm{T}}$ and $[-20,-20,-35,0,-40]^{\mathrm{T}}$, respectively. The other settings and parameters are chosen the same as for the cantilever beam analyzed in Section 2.6.1. For clarity, the FEM mesh of the curved L-shape beam is illustrated in Figure 2.21, which has been constructed by 5000 plane-stress elements equipped with quadratic shape functions, see Eq. 2.23.

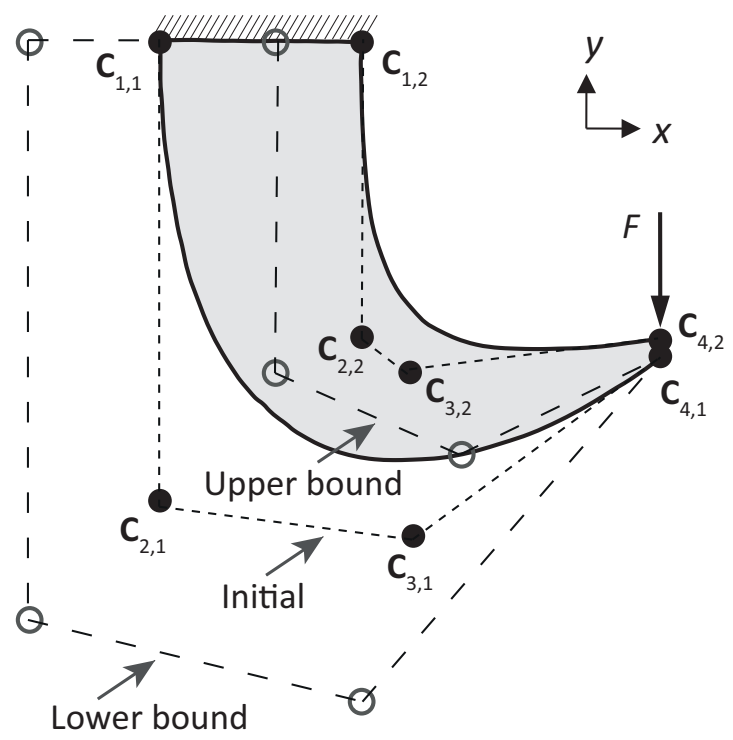

Figure 2.20: Curved L-shape beam with the control points $\mathbf{C}_{i, j}$ and their upper and lower bounds indicated.

Table 2.4: Coordinates $\mathbf{C}_{i, j}$ (in $\mathrm{m}$ ) and weights $\omega_{i, j}$ of control points.

\begin{tabular}{lllll}
\hline$i$ & $j=1$ & $j=2$ \\
\hline 1 & $\mathbf{C}_{1,1}=(-12,0)$ & $\omega_{1,1}=1$ & $\mathbf{C}_{1,2}=(0,0)$ & $\omega_{1,2}=1$ \\
\hline 2 & $\mathbf{C}_{2,1}=(-12,-28)$ & $\omega_{2,1}=1$ & $\mathbf{C}_{2,2}=(0,-18)$ & $\omega_{2,2}=1$ \\
\hline 3 & $\mathbf{C}_{3,1}=(3,-30)$ & $\omega_{3,1}=1$ & $\mathbf{C}_{3,2}=(3,-20)$ & $\omega_{3,2}=1$ \\
\hline 4 & $\mathbf{C}_{3,1}=(18,-19)$ & $\omega_{3,1}=1$ & $\mathbf{C}_{3,2}=(18,-18)$ & $\omega_{3,2}=1$ \\
\hline
\end{tabular}

Figure 2.22 illustrates the final configurations calculated with the coupled topology and domain shape optimization approach using the TSO sequence, Figure 2.22(b), and the STO sequence, Figure 2.22 (c), together with the final configuration computed 


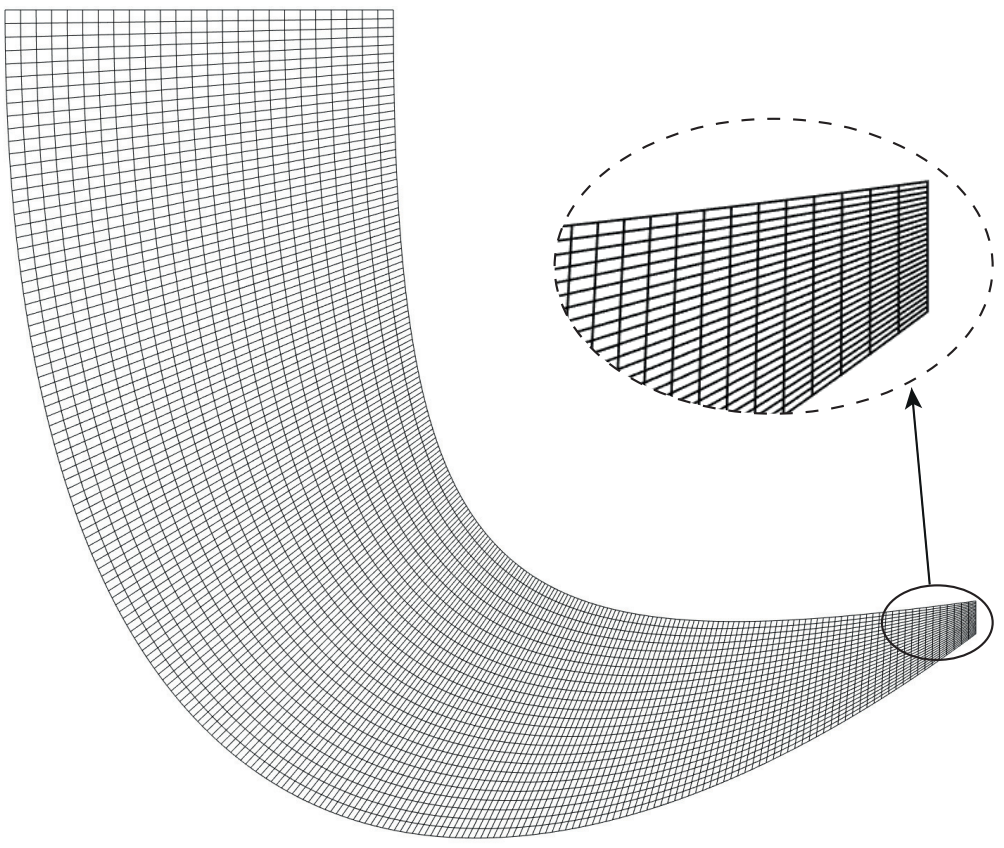

Figure 2.21: Finite element discretization of the final optimized design domain of the curved L-shape beam, with the mesh details at the right side of the beam illustrated in the inset.

with pure topology optimization, Figure 2.22(d). Although the final configurations appear as similar, a comparison of the calculated values of the minimal compliance $c$ illustrates that the coupled optimization approach performed with the STO sequence leads to an optimum $c=146.232 \mathrm{Nmm}$ that lies $10 \%$ below the value found for the topology optimization procedure, $c=161.592 \mathrm{Nmm}$. This is, because for the coupled optimization approach the design space during the computational procedure is slightly altered as a result of the shape optimization process, which allows to find a lower optimum than for pure topology optimization in which the design space is kept fixed. It should be mentioned, though, that the pure topology optimization method possibly may result in a lower structural compliance than the coupled optimization method if the optimization procedure is initiated at the maximal allowable design domain. Nevertheless, the geometry found for pure topology optimization will correspond to an unfavourable, jagged boundary shape for the curved L-shape beam problem. In addition, the value determined from the coupled optimization procedure with the TSO sequence, $c=149.389 \mathrm{Nmm}$, lies $2 \%$ above the value $c=146.232 \mathrm{Nmm}$ calculated with the STO sequence, indicating that the STO update scheme results in a more optimized design than the TSO update scheme. Conversely, as shown in Figure 2.23, the number of iterations used in the outer loop of the TSO update scheme, $k=8$, is considerably lower than for the STO update scheme, $k=20$. In summary, from the 
above comparison study it is concluded that the coupled topology and domain shape optimization method may find a better optimum than a pure topology optimization procedure when the maximal design domain is a priori undefined, since in the coupled optimization method the design domain for the topology optimization step is allowed to change (enlarge).
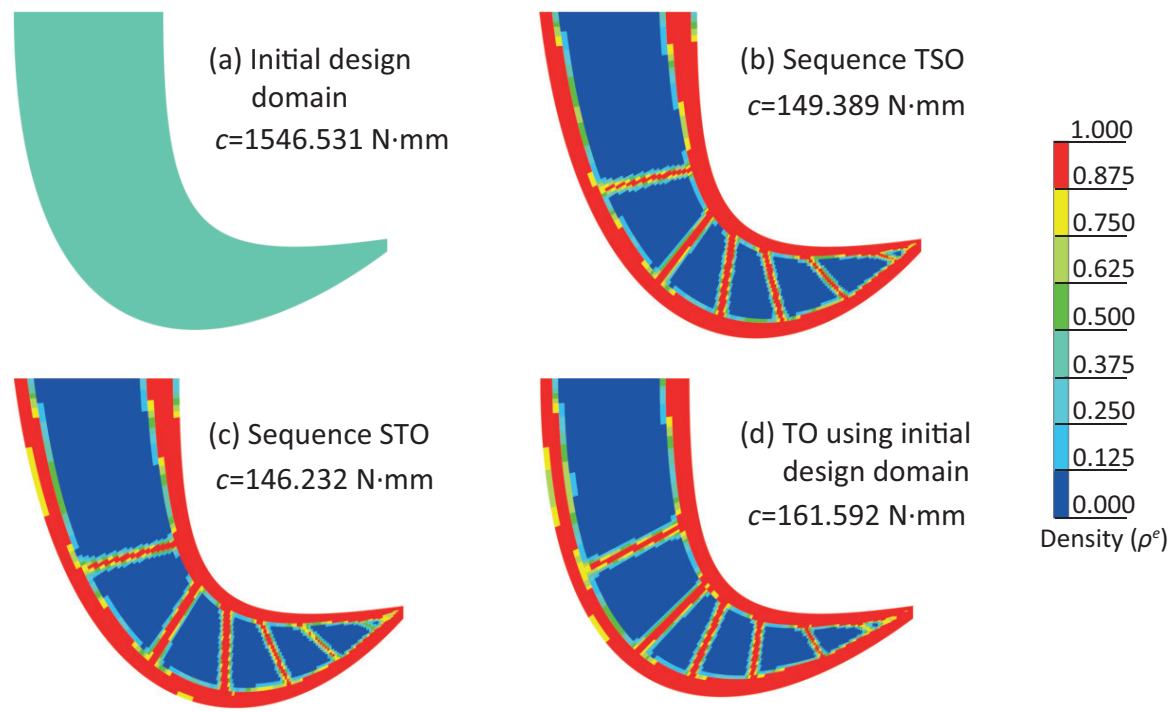

Figure 2.22: Final configurations for the (a) curved L-shape beam, calculated with coupled topology and domain shape optimization using a (b) TSO sequence, and a (c) STO sequence, and computed with (d) pure Topology Optimization (TO) using the initial design domain.

\subsection{Concluding remarks}

In this chapter a modeling framework is presented that couples topology and domain shape optimization. The shape and topology design variables are incrementally updated in a sequential fashion, using a so-called staggered numerical update scheme. Here, the shape of the design domain is momentarily frozen when performing a topology optimization step, and the topology of the design domain is temporarily kept fixed when carrying out a shape optimization step. The sequence of alternating shape and topology optimization steps is repeated until the value of the structural compliance $c$ meets the convergence criteria. The geometry for domain shape optimization is described by NURBS, in order to allow for the analysis of relatively complex structural forms. The domain shape optimization procedure is performed using an SQP method in combination with an efficient gradient-based solution strategy. The topology optimization procedure is carried out in accordance with the SIMP approach.

The usefulness of the coupled optimization approach has been demonstrated by analyzing several benchmark problems. The simulation results confirm a strong depen- 


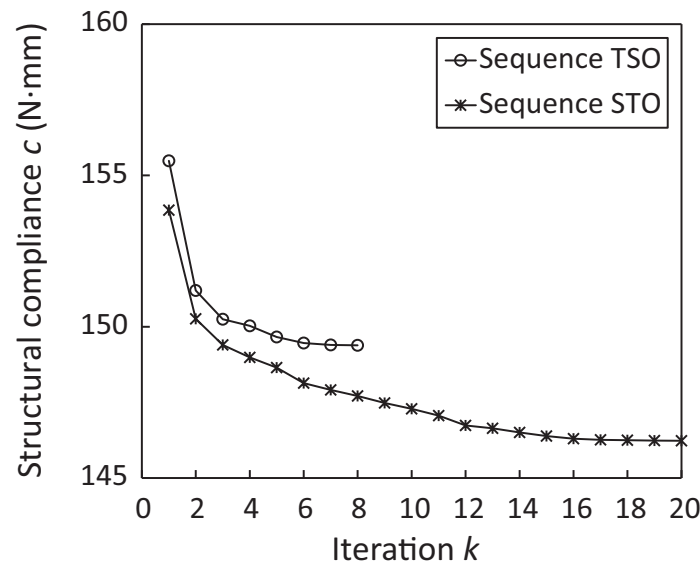

Figure 2.23: Convergence behavior of the structural compliance $c$ for a curved L-shape beam subjected to the coupled optimization approach with TSO and STO sequences.

dency on the initial conditions and bounds selected, showing that a careful construction of the design domain generally is necessary and meaningful. Under the specific conditions whereby the initial design domain has a basic, rectangular shape, of which the size is prescribed by a relatively large value, the effect of domain shape optimization on the computational result may be relatively small, due to which the coupled topology and domain shape optimization framework leads to a similar outcome as that obtained by pure topology optimization. Conversely, the coupled optimization method may find a better optimum than the pure topology optimization method when the maximal design domain is a priori undefined, since in the coupled optimization method the design domain for the topology optimization step is allowed to change (enlarge). The coupled optimization method is a suitable method for the optimization of wind turbines, since it straightforwardly facilitates the incorporation of aerodynamic shape optimization criteria commonly applied in the design of wind turbine rotor blades.

The focus of the present work is on the formulation of a coupled topology and domain shape optimization method, and the demonstration of its main features by means of illustrative numerical benchmark problems. Although the benchmark problems address several numerical discretization effects, such as the mesh sensitivity of the numerical result and the possible appearance of unfavorable element distortions, more research on such aspects is necessary in order to apply the coupled optimization method for advanced three-dimensional structures with complex geometries. Along this line, an interesting topic for future research may be to integrate the current optimization framework with an isogeometric, higher-order discretization method that uses an automated mesh generation procedure. 
2.7. Concluding remarks 


\section{Chapter 3}

\section{Coupled optimization of beam-type structures}

This chapter is based on the journal paper:

Wang, Z., Suiker, A. S. J., Hofmeyer, H., van Hooff, T., Blocken, B., 2020. Sequentially coupled shape and topology optimization for $2.5 \mathrm{D}$ and $3 \mathrm{D}$ beam models (under review).

A sequentially coupled shape and topology optimization framework is presented in which the outer geometry and the internal topological layout of beam-type structures are optimized simultaneously. The outer geometry of the beam-type structures is parametrically described by Non-Uniform Rational B-splines (NURBS), which guarantees a highly accurate description of the structural shape and enable an efficient control of the design domain with only a few control points. The computational efficiency of the coupled optimization approach is assured by applying a gradient-based optimization algorithm, for which the sensitivities are derived in closed form. The formulation of the coupled optimization approach is tailored towards $2.5 \mathrm{D}$ and full $3 \mathrm{D}$ representations of beam structures used in engineering applications. The 2.5D beam model, which has been taken from the literature, uses standard beam elements to simulate the beam response in the longitudinal direction, whereby the cross-sectional properties of the beam elements are calculated from additional 2D Finite Element Method (FEM) analyses. A comparison study of a cantilever beam problem subjected to pure shape optimization and pure topology optimization illustrates that the $2.5 \mathrm{D}$ and $3 \mathrm{D}$ beam models lead to similar shape and topology designs, but that the $2.5 \mathrm{D}$ beam model has a significantly higher computational efficiency. Specifically, the computational times for the 2.5D model are about a factor 70 (shape optimization) and 1.4 (topology optimization) lower than for the 3D model, which indicates that in the coupled 


\subsection{Introduction}

optimization approach the optimization of the shape provides the largest contribution to the higher computational efficiency of the $2.5 \mathrm{D}$ model. The coupled shape and topology optimization analysis subsequently performed on the $2.5 \mathrm{D}$ cantilever beam model demonstrates that the specific order at which the alternating shape and topology optimization increments are performed in the staggered update procedure turns out to have some influence on the computational speed and the value of the minimal compliance computed. Despite these differences, the final beam structures following from the different staggered update procedures illustrate how shape and topology can be efficiently optimized in an integrated, coupled fashion.

\subsection{Introduction}

Beam-type structures are widely used as load-carrying components in the fields of architectural, civil, aerospace and mechanical engineering, among others. Their structural performance can be optimized by minimizing the stresses and displacements generated under the applied loading and boundary conditions, thereby accounting for predefined design constraints on the geometry and the internal material distribution. On the one hand, this requires the application of shape optimization techniques 106 109, in which the outer shape of the structure is parametrized and optimized using shape design variables, such as the spline curves applied for the geometrical description or the nodal coordinates of the underlying Finite Element Method (FEM) model [48]. On the other hand, topology optimization may be applied to optimally place the material within a given design domain, which, for example, can be accomplished by using the so-called SIMP (Solid Isotropic Material with Penalization) approach [43, 66, 68. In this method, the relative material density assigned to each finite element can take values between zero (a void) and unity (a solid), and is directly related to the element stiffness by means of a penalization factor. Correspondingly, the optimized material distribution can be found by minimizing the structural compliance within the design domain.

Due to the nature of the SIMP method, however, the optimization of the material distribution is bounded by the specific choice of the design domain. For obtaining more optimized solutions and to develop insight into the effect of the design domain on the topology optimization procedure, it is necessary that the design domain can vary during the process of topology optimization. This can be accomplished by a coupled shape and topology optimization approach. In addition, in the design of aeroelastic beam-type structures, such as wind turbine blades and aircraft wings, the aerodynamic performance (e.g., lift force or energy output) is usually considered to be the most important design objective [49, 110, 111]. Because the optimization of the aerodynamic performance requires a parametrization of the outer shape of the structure, a coupled shape and topology approach offers a useful tool to simultaneously optimize the aerodynamic and structural performances of aeroelastic beam-type structures. Accordingly, in the present communication a coupled shape and topology optimization formulation is presented for $2.5 \mathrm{D}$ and $3 \mathrm{D}$ beam models, using the optimization framework presented in [112] as a basis. This optimization framework 
incorporates the shape design variables of the design domain in the SIMP topology optimization method, whereby the interactions between shape and topology variations of the design domain are taken into account through performing the corresponding optimization steps in a sequential manner using a so-called staggered update scheme. The optimization process is performed in a computationally efficient fashion by applying a gradient-based optimization algorithm.

Although shape and topology optimization of beam-type structures can be performed using 3D finite element models, for relatively slender beam-type structures, such as wind turbine blades, it is computationally more economical to use the reduced $2.5 \mathrm{D}$ approach proposed in [13 118. In this approach, standard beam elements are employed to simulate the structural response in the longitudinal direction, whereby the cross-sectional properties of the beam element are calculated from additional 2D FEM analyses. The present communication investigates the applicability and performance of $2.5 \mathrm{D}$ beam modeling in the context of coupled shape and topology optimization, by comparing the computational results and efficiency with those obtained from 3D beam models. The geometries of the $2.5 \mathrm{D}$ and $3 \mathrm{D}$ beam models are parametrically described using Non-Uniform Rational B-splines (NURBS) [89, which guarantees a highly accurate description of the shape boundaries by means of smooth basis functions with compact support and enables an efficient control of the design domain with only a few control points [48, 112. In the comparison study of the 3D and 2.5D beam models, for simplicity, the loading applied in the boundary-value problems considered is taken as independent of the beam outer shape. Nevertheless, it is emphasized that the presented optimization framework allows for a relatively straightforward incorporation of shapedependent loads and constraints. For example, the proposed optimization method has been recently applied for the aerostructural design of a Horizontal-Axis Wind Turbine (HAWT) rotor blade, thereby accounting for the shape-dependent aerodynamic loads and rotor power coefficient 119 .

This chapter is organized as follows. Section 3.2 discusses the parametrization of the geometry design model by means of NURBS, thereby distinguishing between 1D curves, 2D surfaces and 3D solids. In Section 3.3 the structural analysis of 3D and $2.5 \mathrm{D}$ beam models is reviewed. The FEM formulations presented in this section are used as input for the derivation of the coupled shape and topology framework provided in Sections 3.4 and 3.5 . whereby Section 3.4 gives the general formulation and Section 3.5 presents the parameter sensitivities defining the gradient-based optimization algorithm. Subsequently, in Section 3.6 the optimization framework is applied in a comparison study of a cantilever beam problem modeled by $2.5 \mathrm{D}$ beam elements and $3 \mathrm{D}$ beam elements. Finally, Section 3.7 provides the main conclusions of the study.

\subsection{Geometry design model}

During the optimization procedure an efficient control of the outer shape of the structure is enabled by using NURBS. Detailed information on the theory of NURBS can be found in 89 . In the present section the formulation of the NURBS curves, surfaces, and solids are provided in matrix form for 1D, 2D and 3D design models, respectively, 
which will be subsequently used in Section 3.5 as input for the analytical derivation of the shape sensitivities.

\subsubsection{NURBS curve}

A NURBS curve is a piecewise polynomial curve composed of B-spline basis functions. The definition of such a basis function is based on a set of non-decreasing real numbers in a parametric space, defined by a so-called knot vector

$$
\boldsymbol{Z}=\left[\zeta_{1}, \zeta_{2}, \ldots, \zeta_{l+r+1}\right]^{\mathrm{T}} \quad \text { with } \quad \zeta_{k} \in \mathbb{R},
$$

where each real number $\zeta_{k}$ reflects a knot with $k$ being the knot index, $l$ is the number of basis functions that comprises the B-spline, and $r$ is the polynomial order of the B-spline. With the knot index $k$ running from 1 to $l$, the B-spline basis functions $L_{k, r}$ are defined recursively for an arbitrarily value of $\zeta$, i.e.,

$$
\begin{aligned}
& \text { for } \quad r=0: \quad L_{k, 0}(\zeta)= \begin{cases}1 & \text { if } \zeta_{k} \leq \zeta<\zeta_{k+1}, \\
0 & \text { otherwise, }\end{cases} \\
& \text { for } \quad r \geq 1: \quad L_{k, r}(\zeta)=\frac{\zeta-\zeta_{k}}{\zeta_{k+r}-\zeta_{k}} L_{k, r-1}(\zeta)+\frac{\zeta_{k+r+1}-\zeta}{\zeta_{k+r+1}-\zeta_{k+1}} L_{k+1, r-1}(\zeta),
\end{aligned}
$$

and can be conveniently stored in a vector as

$$
\mathbf{L}_{\mathrm{b}}=\left[L_{1, r}(\zeta), L_{2, r}(\zeta), \cdots, L_{l, r}(\zeta)\right]^{\mathrm{T}}
$$

The so-called control points define the geometry of the structure, and their locations during the optimization procedure may change. Together with the corresponding weights, the control points are stored in a matrix as

$$
\mathbf{P}_{C}^{1 \mathrm{D}}=\left[\begin{array}{llll}
\omega_{1} C_{1} & \omega_{2} C_{2} & \cdots & \omega_{l} C_{l}
\end{array}\right]^{\mathrm{T}}
$$

where $C_{k} \in\left\{X_{k}, Y_{k}, Z_{k}\right\}$, in which $X_{k}, Y_{k}, Z_{k}$ with $k=1,2, \cdots, l$, are the $x$-, $y$ - and $z$-coordinates of the control points and $\omega_{k}$ is the corresponding weight. Accordingly, the coordinates of an arbitrary point $\mathbf{p}=[x, y, z]^{T}$ of the NURBS curve are determined by

$$
\mathbf{p}^{\mathrm{T}}=\left[\frac{\mathbf{L}_{\mathrm{b}}^{\mathrm{T}} \mathbf{P}_{X}^{1 \mathrm{D}}}{\mathbf{L}_{\mathrm{b}}^{\mathrm{T}} \boldsymbol{\Omega}^{1 \mathrm{D}}}, \frac{\mathbf{L}_{\mathrm{b}}^{\mathrm{T}} \mathbf{P}_{Y}^{1 \mathrm{D}}}{\mathbf{L}_{\mathrm{b}}^{\mathrm{T}} \boldsymbol{\Omega}^{1 \mathrm{D}}}, \frac{\mathbf{L}_{\mathrm{b}}^{\mathrm{T}} \mathbf{P}_{Z}^{1 \mathrm{D}}}{\mathbf{L}_{\mathrm{b}}^{\mathrm{T}} \boldsymbol{\Omega}^{1 \mathrm{D}}}\right],
$$

with the weights of the control points assembled as

$$
\boldsymbol{\Omega}^{1 \mathrm{D}}=\left[\omega_{1}, \omega_{2}, \cdots, \omega_{l}\right]^{\mathrm{T}} .
$$




\subsubsection{NURBS surface}

In order to construct a NURBS surface in a 2D parametric domain, the coordinates characterizing the basis functions are stored in two knot vectors, i.e.,

$$
\boldsymbol{\Xi}=\left[\xi_{1}, \xi_{2}, \ldots, \xi_{n+p+1}\right]^{\mathrm{T}}, \boldsymbol{H}=\left[\eta_{1}, \eta_{2}, \ldots, \eta_{m+q+1}\right]^{\mathrm{T}} \quad \text { with } \quad \xi_{i}, \eta_{j} \in \mathbb{R},
$$

where $i, j$ are the knot indexes, $n, m$ are the number of basis functions and $p, q$ are the polynomial orders. The basis functions $N_{i, p}$ and $M_{j, q}$ in the $\xi$ - and $\eta$-directions can be obtained in a similar fashion as shown in Eq. $(3.2)$. These basis functions are stored in the vectors $\mathbf{N}_{\mathrm{b}}$ and $\mathbf{M}_{\mathrm{b}}$ as

$$
\mathbf{N}_{\mathrm{b}}=\left[N_{1, p}(\xi), N_{2, p}(\xi), \cdots, N_{n, p}(\xi)\right]^{\mathrm{T}},
$$

and

$$
\mathbf{M}_{\mathrm{b}}=\left[M_{1, q}(\eta), M_{2, q}(\eta), \cdots, M_{m, q}(\eta)\right]^{\mathrm{T}} .
$$

For generality, the 2D net of control points defining a NURBS surface is obtained here from the 3D grid of control points describing a NURBS solid. This is done by selecting a single layer of control points from the grid of control points describing the NURBS solid. Accordingly, by considering the directions of the knot vectors $\boldsymbol{\Xi}, \boldsymbol{H}$ and $\boldsymbol{Z}$ in the parametric domain to correspond to the $x$-, $y$ - and $z$-directions in the physical domain, the net of control points becomes

$$
\mathbf{P}_{k, C}^{2 \mathrm{D}}=\left[\begin{array}{cccc}
\omega_{1,1, k} C_{1,1, k} & \omega_{1,2, k} C_{1,2, k} & \cdots & \omega_{1, m, k} C_{1, m, k} \\
\omega_{2,1, k} C_{2,1, k} & \omega_{2,2, k} C_{2,2, k} & \cdots & \omega_{2, m, k} C_{2, m, k} \\
\vdots & \vdots & \ddots & \vdots \\
\omega_{n, 1, k} C_{n, 1, k} & \omega_{n, 2, k} C_{n, 2, k} & \cdots & \omega_{n, m, k} C_{n, m, k}
\end{array}\right]
$$

with $C_{i, j, k} \in\left\{X_{i, j, k}, Y_{i, j, k}, Z_{i, j, k}\right\}$, where $X_{i, j, k}, Y_{i, j, k}$ and $Z_{i, j, k}$ represent the $x$-, $y$ and $z$-coordinates of the control points, respectively. Here, the first two indices vary as $i=1,2, \cdots, n, j=1,2, \cdots, m$, while $k$ is kept fixed as it refers to a specific layer of the $3 \mathrm{D}$ grid of control points. The resulting NURBS surface is defined by the tensor product of the $2 \mathrm{D}$ net of control points and the basis functions $\mathbf{N}_{\mathrm{b}}$ and $\mathbf{M}_{\mathrm{b}}$. As such, an arbitrary point $\mathbf{p}$ of the NURBS surface can be calculated as

$$
\mathbf{p}^{\mathrm{T}}=\left[\frac{\mathbf{N}_{\mathrm{b}}^{\mathrm{T}} \mathbf{P}_{k, X}^{2 \mathrm{D}} \mathbf{M}_{\mathrm{b}}}{\mathbf{N}_{\mathrm{b}}^{\mathrm{T}} \boldsymbol{\Omega}_{k}^{2 \mathrm{D}} \mathbf{M}_{\mathrm{b}}}, \frac{\mathbf{N}_{\mathrm{b}}^{\mathrm{T}} \mathbf{P}_{k, Y}^{2 \mathrm{D}} \mathbf{M}_{\mathrm{b}}}{\mathbf{N}_{\mathrm{b}}^{\mathrm{T}} \boldsymbol{\Omega}_{k}^{2 \mathrm{D}} \mathbf{M}_{\mathrm{b}}}, \frac{\mathbf{N}_{\mathrm{b}}^{\mathrm{T}} \mathbf{P}_{k, Z}^{2 \mathrm{D}} \mathbf{M}_{\mathrm{b}}}{\mathbf{N}_{\mathrm{b}}^{\mathrm{T}} \boldsymbol{\Omega}_{k}^{2 \mathrm{D}} \mathbf{M}_{\mathrm{b}}}\right]
$$

with the weights of the control points as 


$$
\boldsymbol{\Omega}_{k}^{2 \mathrm{D}}=\left[\begin{array}{cccc}
\omega_{1,1, k} & \omega_{1,2, k} & \cdots & \omega_{1, m, k} \\
\omega_{2,1, k} & \omega_{2,2, k} & \cdots & \omega_{2, m, k} \\
\vdots & \vdots & \ddots & \vdots \\
\omega_{n, 1, k} & \omega_{n, 2, k} & \cdots & \omega_{n, m, k}
\end{array}\right]
$$

where $k \in\{1,2, \cdots, l\}$, and $\mathbf{P}_{k, C}^{2 \mathrm{D}}$ follows from Eq. 3.10 .

\subsubsection{NURBS solid}

Although a NURBS solid can be defined in a similar fashion as the NURBS curve and surface, it is relatively straightforward to formulate the NURBS solid by applying projections of NURBS surfaces. Using the 2D control net, Eq. (3.10), and the NURBS surface basis functions $\mathbf{N}_{\mathrm{b}}$ and $\mathbf{M}_{\mathrm{b}}$ given by Eqs. $(3.8)$ and $(3.9)$, a matrix $\mathbf{R}_{C}^{3 \mathrm{D}}$ can be constructed as

$$
\mathbf{R}_{C}^{3 \mathrm{D}}=\left[\mathbf{N}_{\mathrm{b}}^{\mathrm{T}} \mathbf{P}_{1, C}^{2 \mathrm{D}} \mathbf{M}_{\mathrm{b}}, \mathbf{N}_{\mathrm{b}}^{\mathrm{T}} \mathbf{P}_{2, C}^{2 \mathrm{D}} \mathbf{M}_{\mathrm{b}}, \cdots, \mathbf{N}_{\mathrm{b}}^{\mathrm{T}} \mathbf{P}_{l, C}^{2 \mathrm{D}} \mathbf{M}_{\mathrm{b}}\right]^{\mathrm{T}},
$$

where $C \in\{X, Y, Z\}$ represents the $x$-, $y$ - and $z$-coordinates of the control points. With Eq. 3.13, an arbitrary point $\mathbf{p}$ of the NURBS solid is described by

$$
\mathbf{p}^{\mathrm{T}}=\left[\frac{\mathbf{L}_{\mathrm{b}}^{\mathrm{T}} \mathbf{R}_{X}^{3 \mathrm{D}}}{\mathbf{L}_{\mathrm{b}}^{\mathrm{T}} \boldsymbol{\Omega}^{3 \mathrm{D}}}, \frac{\mathbf{L}_{\mathrm{b}}^{\mathrm{T}} \mathbf{R}_{Y}^{3 \mathrm{D}}}{\mathbf{L}_{\mathrm{b}}^{\mathrm{T}} \boldsymbol{\Omega}^{3 \mathrm{D}}}, \frac{\mathbf{L}_{\mathrm{b}}^{\mathrm{T}} \mathbf{R}_{Z}^{3 \mathrm{D}}}{\mathbf{L}_{\mathrm{b}}^{\mathrm{T}} \boldsymbol{\Omega}^{3 \mathrm{D}}}\right],
$$

with

$$
\boldsymbol{\Omega}^{3 \mathrm{D}}=\left[\mathbf{N}_{\mathrm{b}}^{\mathrm{T}} \boldsymbol{\Omega}_{1}^{2 \mathrm{D}} \mathbf{M}_{\mathrm{b}}, \mathbf{N}_{\mathrm{b}}^{\mathrm{T}} \boldsymbol{\Omega}_{2}^{2 \mathrm{D}} \mathbf{M}_{\mathrm{b}}, \cdots, \mathbf{N}_{\mathrm{b}}^{\mathrm{T}} \boldsymbol{\Omega}_{l}^{2 \mathrm{D}} \mathbf{M}_{\mathrm{b}}\right]^{\mathrm{T}}
$$

where $\boldsymbol{\Omega}_{k}^{2 \mathrm{D}}$ (with $k=1,2, \cdots, l$ ) is defined by Eq. $(3.12)$, and $\mathbf{N}_{\mathrm{b}}^{\mathrm{T}}, \mathbf{M}_{\mathrm{b}}^{\mathrm{T}}$ and $\mathbf{L}_{\mathrm{b}}^{\mathrm{T}}$ are the basis functions in the three directions of the NURBS solid.

\subsection{Structural analysis model}

Consider the 3D beam-type structure shown in Figure 3.1(a), which will be used for demonstrating specific features of the shape and topology optimization procedure. The beam is modeled by means of the FEM, whereby two types of element discretization are adopted, namely i) a discretization by $3 \mathrm{D}$ continuum elements, see Figure 3.1 (b), and ii) a discretization by 1D beam elements in the longitudinal direction of the beam, combined with additional 2D continuum elements for a detailed modeling of the crosssectional properties of a beam element, see Figure 3.1(c). For brevity, in the following these two types of discretization will be denoted as the "3D beam model" and the "2.5D beam model", respectively. 


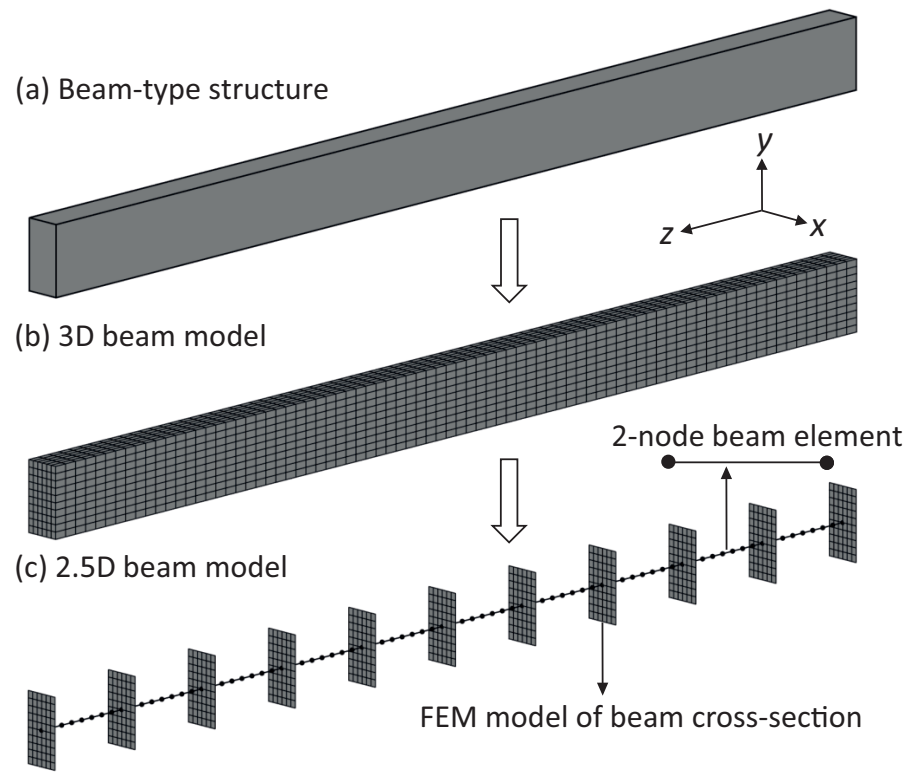

Figure 3.1: A slender beam structure (a), modeled as a $3 \mathrm{D}$ configuration (b) and a 2.5D configuration (c).

The finite element discretizations for the 3D and 2.5D beam models are obtained by projecting a fixed, auxiliary mesh, defined in the parametric domain, onto the actual mesh in the physical domain, using Eqs. (3.5), (3.11), and (3.14) for 1D, $2 \mathrm{D}$ and $3 \mathrm{D}$ finite elements. This projection is schematically illustrated in Figure 3.2 , and enables obtaining an explicit relationship between the structural response of the beam structure and the parameters that control the geometry, i.e., the NURBS control points. Accordingly, analytical expressions can be derived for the parameter sensitivities governing the structural shape optimization, which will be presented in Section 3.5

\subsubsection{D beam model}

Given the knot vectors, the control points with their weights, and a fixed auxiliary mesh in the parametric domain, the discretization of the $3 \mathrm{D}$ finite element model is carried out by projecting the nodal coordinates $\left(\xi_{n}, \eta_{n}, \zeta_{n}\right)$ of the fixed, auxiliary mesh onto the nodal coordinates $\left(x_{n}, y_{n}, z_{n}\right)$ of the actual mesh in the physical domain, in correspondence with Eq. (3.14). Hence, for each isoparametric element $e$, its nodal coordinates can be cast into a vector:

$$
\overline{\mathbf{p}}_{e}=\left[\mathbf{p}_{1}^{\mathrm{T}}, \mathbf{p}_{2}^{\mathrm{T}}, \cdots, \mathbf{p}_{n_{e}}^{\mathrm{T}}\right]^{\mathrm{T}},
$$




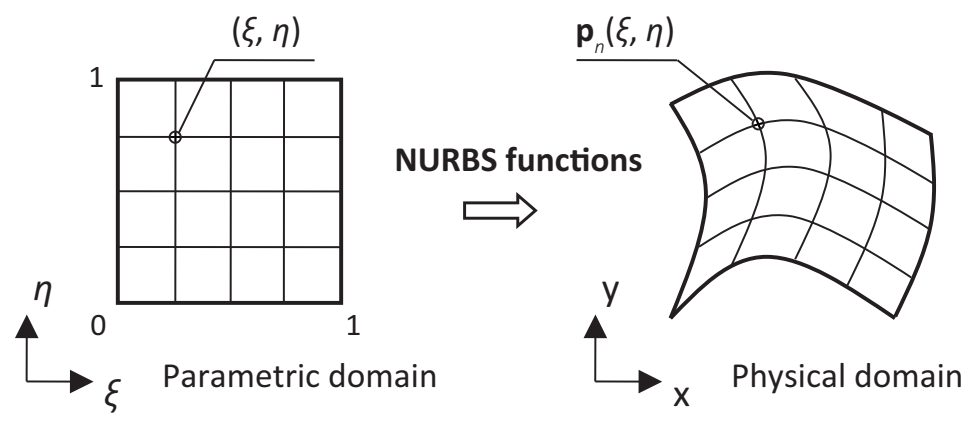

Figure 3.2: Parameterization of the physical design domain via a coordinate projection from the parametric domain. The solid lines in the parametric domain define a fixed auxiliary mesh, which is projected by means of NURBS functions onto the actual finite element mesh that discretizes the physical design domain.

where the coordinates $\mathbf{p}_{n}$ of node $n$, with $n=1,2, \cdots, n_{e}$, are obtained from Eq. (3.14), with $n_{e}$ the total number of nodes of element $e$. The location of an arbitrary point $\hat{\mathbf{p}}_{e}=[x, y, z]^{\mathrm{T}}$ within the finite element $e$ can be obtained from an interpolation of the coordinates of the element nodes $\overline{\mathbf{p}}_{e}$ as:

$$
\hat{\mathbf{p}}_{e}=\mathbf{N}_{e} \overline{\mathbf{p}}_{e}
$$

with

$$
\mathbf{N}_{e}=\left[\begin{array}{llll}
N_{1} \mathbf{I}_{d} & N_{2} \mathbf{I}_{d} & \cdots & N_{n_{e}} \mathbf{I}_{d}
\end{array}\right]
$$

where $N_{n}$, with $n=1,2, \cdots, n_{e}$, are the polynomial interpolation functions of the element, and $\mathbf{I}_{d}$ represents the $d \times d$ identity matrix, with $d$ the number of degrees of freedom of the element node. By introducing the gradient operator $\boldsymbol{\partial}=$ $\left[\partial / \partial \xi_{e}, \partial / \partial \eta_{e}, \partial / \partial \zeta_{e}\right]^{\mathrm{T}}$, the Jacobian matrix of element $e$ follows as

$$
\mathbf{J}_{e}=\boldsymbol{\partial} \hat{\mathbf{p}}_{e}^{\mathrm{T}}
$$

Inserting Eq. (3.17) into Eq. (3.19), the Jacobian matrix $\mathbf{J}_{e}$ can be directly expressed in terms of the nodal coordinates $\overline{\mathbf{p}}_{e}$, i.e.,

$$
\mathbf{J}_{e}=\overline{\mathbf{P}}_{e}\left(\partial \mathbf{N}_{e}^{\mathrm{T}}\right)
$$

with 


$$
\overline{\mathbf{P}}_{e}=\left[\begin{array}{ccc}
\overline{\mathbf{p}}_{e}^{\mathrm{T}} & \mathbf{0} & \mathbf{0} \\
\mathbf{0} & \overline{\mathbf{p}}_{e}^{\mathrm{T}} & \mathbf{0} \\
\mathbf{0} & \mathbf{0} & \overline{\mathbf{p}}_{e}^{\mathrm{T}}
\end{array}\right],
$$

where $\overline{\mathbf{p}}_{e}$ is given by Eq. 3.16. When denoting the displacement vector for an arbitrary point of the structure as $\mathbf{s}=\left[s_{x}, s_{y}, s_{z}\right]^{\mathrm{T}}$, the corresponding strain vector $\boldsymbol{\epsilon}=\left[\epsilon_{x x}, \epsilon_{y y}, 2 \epsilon_{x y}, 2 \epsilon_{x z}, 2 \epsilon_{y z}, \epsilon_{z z}\right]^{\mathrm{T}}$ can be calculated as $\boldsymbol{\epsilon}=\mathbf{B s}$, with the matrix $\mathbf{B}$ given by:

$$
\mathbf{B}=\left[\begin{array}{cccccc}
\partial / \partial x & 0 & \partial / \partial y & \partial / \partial z & 0 & 0 \\
0 & \partial / \partial y & \partial / \partial x & 0 & \partial / \partial z & 0 \\
0 & 0 & 0 & \partial / \partial x & \partial / \partial y & \partial / \partial z
\end{array}\right]^{\mathrm{T}}
$$

At the element level, the matrix $\mathbf{B}_{e}$ can be expressed as [118]

$$
\mathbf{B}_{e}=\mathbf{B}_{e}^{1} \frac{\partial}{\partial \xi_{e}}+\mathbf{B}_{e}^{2} \frac{\partial}{\partial \eta_{e}}+\mathbf{B}_{e}^{3} \frac{\partial}{\partial \zeta_{e}},
$$

with

$$
\mathbf{B}_{e}^{c}=\left[\begin{array}{ccc}
\left(\mathbf{J}_{e}^{-1}\right)_{1 c} & 0 & 0 \\
0 & \left(\mathbf{J}_{e}^{-1}\right)_{2 c} & 0 \\
\left(\mathbf{J}_{e}^{-1}\right)_{2 c} & \left(\mathbf{J}_{e}^{-1}\right)_{1 c} & 0 \\
\left(\mathbf{J}_{e}^{-1}\right)_{3 c} & 0 & \left(\mathbf{J}_{e}^{-1}\right)_{1 c} \\
0 & \left(\mathbf{J}_{e}^{-1}\right)_{3 c} & \left(\mathbf{J}_{e}^{-1}\right)_{2 c} \\
0 & 0 & \left(\mathbf{J}_{e}^{-1}\right)_{3 c}
\end{array}\right] \quad \text { with } \quad c \in\{1,2,3\}
$$

where the index $c$ refers to the column of the inverse of the Jacobian matrix, Eq. 3.20). Using Eqs. 3.16 to 3.24, the stiffness matrix $\mathbf{k}_{e}$ of the isoparametric 3D element is computed as

$$
\mathbf{k}_{e}=\int_{-1}^{1} \int_{-1}^{1} \int_{-1}^{1}\left(\mathbf{B}_{e} \mathbf{N}_{e}\right)^{\mathrm{T}} \mathbf{Q}_{e} \mathbf{B}_{e} \mathbf{N}_{e}\left|\mathbf{J}_{e}\right| d \xi_{e} d \eta_{e} d \zeta_{e},
$$

with $\left|\mathbf{J}_{e}\right|$ the determinant of the Jacobian matrix $\mathbf{J}_{e}$. Here, the $6 \times 6$ matrix $\mathbf{Q}_{e}$ incorporates the constitutive properties of the element. In accordance with the SIMP approach used for topology optimization, the stiffness matrix is scaled by the relative density of the element via a power-law expression [43, 66, 68.

$$
\mathbf{Q}_{e}=\left(\rho_{e}\right)^{p} \mathbf{Q},
$$

where $\rho_{e}$ is the relative density of element $e, p$ is a penalization factor with a typical value being 3 [43], and $\mathbf{Q}$ represents the constitutive behavior in a material point taken here as linear-elastic - in accordance with $\sigma=\mathbf{Q} \boldsymbol{\epsilon}$, with the stress given by 
$\boldsymbol{\sigma}=\left[\sigma_{x x}, \sigma_{y y}, \sigma_{x y}, \sigma_{x z}, \sigma_{y z}, \sigma_{z z}\right]^{\mathrm{T}}$. Finally, the global stiffness matrix $\mathbf{K}$ is found by assembling the element stiffness matrices:

$$
\mathbf{K}=\sum_{e=1}^{N} \mathbf{k}_{e}
$$

with $N$ the total number of elements and the sum operator representing the assembly procedure typically used in the finite element method. The global displacement vector $\mathbf{u}$ is obtained by solving the system of equilibrium equations

$$
\mathbf{K u}=\mathbf{f}
$$

where $\mathbf{f}$ is the global force vector.

\subsection{D beam model}

For the 2.5D beam model, standard 1D beam elements are used to discretize the beam in the longitudinal direction, while the cross-sectional properties of the beam elements are calculated from additional 2D FEM analyses. In this section, the 2.5D FEM approach originally presented in 113 is reviewed, see also [114 118 for more details; these expressions serve as input for the computation of the shape and topology sensitivities used in the gradient-based optimization algorithm, see Section 3.5

\subsubsection{Beam finite element model}

In the beam FEM model the deformations are described by translations $\chi=\left[\chi_{x}, \chi_{y}, \chi_{z}\right]^{\mathrm{T}}$ and rotations $\varphi=\left[\varphi_{x}, \varphi_{y}, \varphi_{z}\right]^{\mathrm{T}}$ of a cross-sectional reference point. These deformations are grouped into a single vector as $\mathbf{r}=\left[\chi^{\mathrm{T}}, \varphi^{\mathrm{T}}\right]^{\mathrm{T}}$. In addition, the shear strains in the $x$ - and $y$-directions and the normal strain in the $z$-direction are assembled as $\tau=\left[\tau_{x}, \tau_{y}, \tau_{z}\right]^{\mathrm{T}}$, and the curvatures about the three directions are given by $\boldsymbol{\kappa}=\left[\kappa_{x}, \kappa_{y}, \kappa_{z}\right]^{\mathrm{T}}$. The above two deformation measures can be assembled into a single vector as $\boldsymbol{\psi}=\left[\boldsymbol{\tau}^{\mathrm{T}}, \boldsymbol{\kappa}^{\mathrm{T}}\right]^{\mathrm{T}}$. Accordingly, the strain-displacement relation becomes $\boldsymbol{\psi}=\overline{\mathbf{B}} \mathbf{r}$, with the operator $\overline{\mathbf{B}}$ given by

$$
\overline{\mathbf{B}}=\mathbf{T}_{r}+\mathbf{I}_{6} \frac{\partial}{\partial z}
$$

where

$$
\mathbf{T}_{r}=\left[\begin{array}{ll}
\mathbf{0}_{3} & \mathbf{t}_{r} \\
\mathbf{0}_{3} & \mathbf{0}_{3}
\end{array}\right] \quad \text { and } \quad \mathbf{t}_{r}=\left[\begin{array}{ccc}
0 & -1 & 0 \\
1 & 0 & 0 \\
0 & 0 & 0
\end{array}\right]
$$

Further, $\mathbf{I}_{6}$ is the $6 \times 6$ identity matrix, $\mathbf{0}_{3}$ is the $3 \times 3$ matrix null matrix, and the $z$-direction corresponds to the longitudinal direction of the beam, see Figure 3.1 
From the strains and curvatures, the normal force and shear forces $\mathbf{T}=\left[T_{x}, T_{y}, T_{z}\right]^{\mathrm{T}}$ and bending moment and torsional moments $\mathbf{M}=\left[M_{x}, M_{y}, M_{z}\right]^{\mathrm{T}}$ can be determined as follows:

$$
\boldsymbol{\theta}=\mathbf{K}^{\mathrm{s}} \boldsymbol{\psi}
$$

with $\boldsymbol{\theta}=\left[\mathbf{T}^{\mathrm{T}}, \mathbf{M}^{\mathrm{T}}\right]^{\mathrm{T}}$ and $\mathbf{K}^{\mathrm{s}}$ representing the stiffness matrix of the beam crosssection.

Analogous to Eq. (3.28) for a 3D beam model, the equilibrium equations for a $2.5 \mathrm{D}$ beam model are defined by

$$
\overline{\mathbf{K}} \overline{\mathbf{u}}=\mathbf{f},
$$

with

$$
\overline{\mathbf{K}}=\sum_{e=1}^{N} \mathbf{k}_{e}^{\mathrm{b}}
$$

where the stiffness $\mathbf{k}_{e}^{\mathrm{b}}$ of the $1 \mathrm{D}$ beam element is computed as

$$
\mathbf{k}_{e}^{\mathrm{b}}=\int_{-1}^{1}\left(\overline{\mathbf{B}}_{e} \mathbf{N}_{e}\right)^{\mathrm{T}} \mathbf{K}_{e}^{\mathrm{s}} \overline{\mathbf{B}}_{e} \mathbf{N}_{e}\left|\overline{\mathbf{J}}_{e}\right| d \zeta_{e} .
$$

Here, $\overline{\mathbf{K}}$ is the global stiffness matrix, $\overline{\mathbf{u}}$ contains the nodal displacements and rotations of the beam, $\mathbf{N}_{e}$ includes the interpolation functions for the beam displacements and rotations, the operator $\mathbf{B}_{e}$ for each element $e$ is given by Eq. $(3.29)$, and $\left|\overline{\mathbf{J}}_{e}\right|$ represents the determinant of the Jacobian matrix, with $\overline{\mathbf{J}}_{e}$ expressed as

$$
\overline{\mathbf{J}}_{e}=\left[\begin{array}{ccc}
1 & 0 & 0 \\
0 & 1 & 0 \\
0 & 0 & \frac{\partial z}{\partial \zeta_{e}}
\end{array}\right]
$$

where $\partial z / \partial \zeta_{e}$ is calculated from Eq. (3.17) using the beam interpolation functions. The derivation of the cross-sectional stiffness matrix $\mathbf{K}_{e}^{\mathrm{s}}$ of the beam element, which appears in Eq. (3.34), is discussed in Section 3.3.2.2 below.

\subsubsection{Cross-sectional stiffness}

The displacements $\mathbf{s}$ of an arbitrary point of the beam cross-section can be expressed in terms of the rigid-body displacements and rotations $\mathbf{r}$ of the cross-sectional reference point, complemented by the in-plane and out-of-plane warping displacements $\mathbf{s}^{\mathrm{w}}$ associated to the deformation of the cross-section, i.e.,

$$
\mathbf{s}=\mathbf{Z r}+\mathbf{s}^{\mathrm{w}}
$$


with

$$
\mathbf{Z}=\left[\begin{array}{cccccc}
1 & 0 & 0 & 0 & z & -y \\
0 & 1 & 0 & -z & 0 & x \\
0 & 0 & 1 & y & -x & 0
\end{array}\right]
$$

where $x, y$, and $z$ are the coordinates of the specific location in the cross-section, with $x$ and $y$ defined with respect to the location of the cross-sectional reference point. Using a FEM model for the cross-section, the warping displacements $\mathbf{s}^{\mathrm{w}}$ are discretized as

$$
\mathbf{s}^{\mathrm{w}}=\mathbf{N} \hat{\mathbf{u}}
$$

where $\mathbf{N}$ contains the interpolation functions of the cross-sectional finite element and $\hat{\mathbf{u}}$ represents the vector with the nodal warping displacements of the element. As will be shown below, it is convenient to isolate the partial derivative terms $\partial / \partial z$ in the strain-displacement relation, which is done by combining $\epsilon=$ Bs with Eq. 3.22, resulting in

$$
\boldsymbol{\epsilon}=\hat{\mathbf{B}} \mathbf{s}+\mathbf{S} \frac{\partial \mathbf{s}}{\partial z}
$$

with

$$
\hat{\mathbf{B}}=\left[\begin{array}{cccccc}
\partial / \partial x & 0 & \partial / \partial y & 0 & 0 & 0 \\
0 & \partial / \partial y & \partial / \partial x & 0 & 0 & 0 \\
0 & 0 & 0 & \partial / \partial x & \partial / \partial y & 0
\end{array}\right]^{\mathrm{T}}
$$

and

$$
\mathbf{S}=\left[\begin{array}{llllll}
0 & 0 & 0 & 1 & 0 & 0 \\
0 & 0 & 0 & 0 & 1 & 0 \\
0 & 0 & 0 & 0 & 0 & 1
\end{array}\right]^{\mathrm{T}}
$$

Substituting Eq. (3.38) into Eq. (3.36), and inserting the result into Eq. (3.39) leads to

$$
\boldsymbol{\epsilon}=\mathbf{S Z}\left(\mathbf{T}_{r}+\mathbf{I}_{6} \frac{\partial}{\partial z}\right) \mathbf{r}+\hat{\mathbf{B}} \mathbf{N} \hat{\mathbf{u}}+\mathbf{S N} \frac{\partial \hat{\mathbf{u}}}{\partial z}
$$

which uses the relation [118]

$$
\hat{\mathbf{B Z}}=\mathbf{S Z T}_{r},
$$


with $\mathbf{T}_{r}$ given by Eq. 3.30 . Invoking the kinematic relation $\boldsymbol{\psi}=\overline{\mathbf{B}} \mathbf{r}$ with $\overline{\mathbf{B}}$ given by Eq. 3.29, Eq. (3.42) finally turns into

$$
\boldsymbol{\epsilon}=\mathbf{S Z} \boldsymbol{\psi}+\hat{\mathbf{B}} \mathbf{N} \hat{\mathbf{u}}+\mathbf{S N} \frac{\partial \hat{\mathbf{u}}}{\partial z} .
$$

As a next step, the equilibrium equations for the beam cross-section need to be formulated. In accordance with the variational framework presented in 115 118, equilibrium is described in terms of $\hat{\mathbf{u}}, \boldsymbol{\psi}$ and $\boldsymbol{\theta}$ by the following set of partial differential equations:

$$
\left\{\begin{array}{l}
\mathbf{M} \frac{\partial^{2} \hat{\mathbf{u}}}{\partial z^{2}}+\left(\mathbf{C}-\mathbf{C}^{\mathrm{T}}\right) \frac{\partial \hat{\mathbf{u}}}{\partial z}+\mathbf{L} \frac{\partial \boldsymbol{\psi}}{\partial z}-\mathbf{E} \hat{\mathbf{u}}-\mathbf{R} \boldsymbol{\psi}=\mathbf{0} \\
\mathbf{L}^{\mathrm{T}} \frac{\partial \hat{\mathbf{u}}}{\partial z}+\mathbf{R}^{\mathrm{T}} \hat{\mathbf{u}}+\mathbf{A} \boldsymbol{\psi}=\boldsymbol{\theta} \\
\frac{\partial \boldsymbol{\theta}}{\partial z}=\mathbf{T}_{r}^{\mathrm{T}} \boldsymbol{\theta}
\end{array}\right.
$$

with

$$
\begin{aligned}
& \mathbf{A}=\sum_{e=1}^{N} \int_{-1}^{1} \int_{-1}^{1}\left(\mathbf{S}_{e} \mathbf{Z}_{e}\right)^{\mathrm{T}} \mathbf{Q}_{e} \mathbf{S}_{e} \mathbf{Z}_{e}\left|\hat{\mathbf{J}}_{e}\right| d \xi_{e} d \eta_{e}, \\
& \mathbf{R}=\sum_{e=1}^{N} \int_{-1}^{1} \int_{-1}^{1}\left(\hat{\mathbf{B}}_{e} \mathbf{N}_{e}\right)^{\mathrm{T}} \mathbf{Q}_{e} \mathbf{S}_{e} \mathbf{Z}_{e}\left|\hat{\mathbf{J}}_{e}\right| d \xi_{e} d \eta_{e} \\
& \mathbf{E}=\sum_{e=1}^{N} \int_{-1}^{1} \int_{-1}^{1}\left(\hat{\mathbf{B}}_{e} \mathbf{N}_{e}\right)^{\mathrm{T}} \mathbf{Q}_{e} \hat{\mathbf{B}}_{e} \mathbf{N}_{e}\left|\hat{\mathbf{J}}_{e}\right| d \xi_{e} d \eta_{e}, \\
& \mathbf{C}=\sum_{e=1}^{N} \int_{-1}^{1} \int_{-1}^{1}\left(\mathbf{S}_{e} \mathbf{N}_{e}\right)^{\mathrm{T}} \mathbf{Q}_{e} \hat{\mathbf{B}}_{e} \mathbf{N}_{e}\left|\hat{\mathbf{J}}_{e}\right| d \xi_{e} d \eta_{e} \\
& \mathbf{L}=\sum_{e=1}^{N} \int_{-1}^{1} \int_{-1}^{1}\left(\mathbf{S}_{e} \mathbf{N}_{e}\right)^{\mathrm{T}} \mathbf{Q}_{e} \mathbf{S}_{e} \mathbf{Z}_{e}\left|\hat{\mathbf{J}}_{e}\right| d \xi_{e} d \eta_{e} \\
& \mathbf{M}=\sum_{e=1}^{N} \int_{-1}^{1} \int_{-1}^{1}\left(\mathbf{S}_{e} \mathbf{N}_{e}\right)^{\mathrm{T}} \mathbf{Q}_{e} \mathbf{S}_{e} \mathbf{N}_{e}\left|\hat{\mathbf{J}}_{e}\right| d \xi_{e} d \eta_{e}
\end{aligned}
$$

Note that the generalized loads $\boldsymbol{\theta}$ given by Eq. (3.31) are related to the stress components by $\boldsymbol{\theta}=\int_{A} \mathbf{Z}^{\mathrm{T}} \boldsymbol{\sigma}_{b} d A$, with $\mathbf{Z}$ given by Eq. (3.37) and the stress $\boldsymbol{\sigma}_{b}$ presented by $\boldsymbol{\sigma}_{b}=\left[\sigma_{x z}, \sigma_{y z}, \sigma_{z z}\right]^{\mathrm{T}}$. Further, the matrices $\mathbf{A}, \mathbf{R}, \mathbf{E}, \mathbf{C}, \mathbf{L}$ and $\mathbf{M}$ presented by Eq. (3.46) are determined from the FEM model at cross-sectional level. In Eq. (3.46), the matrix $\mathbf{Z}_{e}$ is obtained from Eq. (3.37), whereby the $x$ - and $y$-coordinates within a cross-sectional element are calculated from combining the element interpolation functions with the nodal coordinates of the element. The nodal coordinates of an arbitrary cross-sectional finite element $e$ are computed via Eq. [3.16), where $\mathbf{p}_{n}$ represents the nodal coordinates determined from Eq. 3.11, with the z-coordinate of the control points $Z_{i, j, k}$ in Eq. 3.10 set to zero. Furthermore, the matrix $\mathbf{S}_{e}$ is computed from 
Eq. 3.41, and $\mathbf{Q}_{e}$ follows from Eq. 3.26), in which $\rho_{e}$ refers to the relative material density in finite element $e$, and $\left|\hat{\mathbf{J}}_{e}\right|$ is the determinant of the Jacobian matrix of the element. The Jacobian matrix has the usual form

$$
\hat{\mathbf{J}}_{e}=\left[\begin{array}{ccc}
\frac{\partial x}{\partial \xi_{e}} & \frac{\partial y}{\partial \xi_{e}} & 0 \\
\frac{\partial x}{\partial \eta_{e}} & \frac{\partial y}{\partial \eta_{e}} & 0 \\
0 & 0 & 1
\end{array}\right]
$$

which, by invoking Eqs. 3.10, 3.11, 3.16, 3.17), 3.18 and (3.19, can be expressed as a function of the element nodal coordinates (and thus as function of the NURBS control points):

$$
\hat{\mathbf{J}}_{e}=\hat{\boldsymbol{\partial}} \hat{\mathbf{p}}^{\mathrm{T}}+\hat{\mathbf{J}}_{\zeta}=\overline{\mathbf{P}}_{e}\left(\hat{\boldsymbol{\partial}} \mathbf{N}_{e}^{\mathrm{T}}\right)+\hat{\mathbf{J}}_{\zeta} \quad \text { with } \quad \hat{\mathbf{J}}_{\zeta}=\left[\begin{array}{ccc}
0 & 0 & 0 \\
0 & 0 & 0 \\
0 & 0 & 1
\end{array}\right]
$$

with the derivative operator $\hat{\boldsymbol{\partial}}=\left[\partial / \partial \xi_{e}, \partial / \partial \eta_{e}, 0\right]^{\mathrm{T}}$ and the matrix $\overline{\mathbf{P}}_{e}$ given by Eq. (3.21). After computing the inverse of the Jacobian matrix Eq. 3.48 , the matrix $\hat{\mathbf{B}}_{e}$ appearing in Eq. 3.46 can be developed from Eq. 3.40 as

$$
\hat{\mathbf{B}}_{e}=\hat{\mathbf{B}}_{e}^{1} \frac{\partial}{\partial \xi_{e}}+\hat{\mathbf{B}}_{e}^{2} \frac{\partial}{\partial \eta_{e}},
$$

where

$$
\hat{\mathbf{B}}_{e}^{c}=\left[\begin{array}{ccc}
\left(\hat{\mathbf{J}}_{e}^{-1}\right)_{1 c} & 0 & 0 \\
0 & \left(\hat{\mathbf{J}}_{e}^{-1}\right)_{2 c} & 0 \\
\left(\hat{\mathbf{J}}_{e}^{-1}\right)_{2 c} & \left(\hat{\mathbf{J}}_{e}^{-1}\right)_{1 c} & 0 \\
0 & 0 & \left(\hat{\mathbf{J}}_{e}^{-1}\right)_{1 c} \\
0 & 0 & \left(\hat{\mathbf{J}}_{e}^{-1}\right)_{2 c} \\
0 & 0 & 0
\end{array}\right] \quad \text { with } \quad c \in\{1,2\}
$$

For solving the set of differential equations, Eq. (3.45), the contribution by the rigid body motions $\mathbf{r}$ needs to be removed, which enters Eq. 3.45 via the warping displacements $\hat{\mathbf{u}}$. The rigid body motions can be eliminated by satisfying the constraint equations [115]

$$
\left[\begin{array}{cc}
\mathbf{D}^{\mathrm{T}} & \mathbf{0} \\
\mathbf{0} & \mathbf{D}^{\mathrm{T}}
\end{array}\right]\left[\begin{array}{c}
\hat{\mathbf{u}} \\
\frac{\partial \hat{\mathbf{u}}}{\partial z}
\end{array}\right]=\left[\begin{array}{l}
\mathbf{0} \\
\mathbf{0}
\end{array}\right]
$$

with

$$
\mathbf{D}=\left[\begin{array}{llll}
\mathbf{Z}_{1}^{\mathrm{T}}, & \mathbf{Z}_{2}^{\mathrm{T}}, & \cdots, & \mathbf{Z}_{n_{n}}^{\mathrm{T}}
\end{array}\right]^{\mathrm{T}}
$$


where $n_{n}$ is the total number of element nodes in the FEM mesh and $\mathbf{Z}_{n}$ is obtained by inserting the nodal coordinates of node $n$ in Eq. (3.37). Formulating Eq. (3.45) in matrix-vector notation and incorporating the constraint relations, Eq. 3.51), results in the system of equilibrium equations [117]

$$
\hat{\mathbf{K}} \mathbf{w}=\hat{\mathbf{f}} \Longleftrightarrow\left[\begin{array}{cc}
\mathbf{K}_{11} & \left(\mathbf{H}^{\mathrm{T}}-\mathbf{H}\right) \\
\mathbf{0} & \mathbf{K}_{11}
\end{array}\right]\left[\begin{array}{l}
\mathbf{w}_{1} \\
\mathbf{w}_{2}
\end{array}\right]=\left[\begin{array}{l}
\mathbf{f}_{1} \\
\mathbf{f}_{2}
\end{array}\right],
$$

with

$$
\mathbf{K}_{11}=\left[\begin{array}{ccc}
\mathbf{E} & \mathbf{R} & \mathbf{D} \\
\mathbf{R}^{\mathrm{T}} & \mathbf{A} & \mathbf{0} \\
\mathbf{D}^{\mathrm{T}} & \mathbf{0} & \mathbf{0}
\end{array}\right] \quad \text { and } \mathbf{H}=\left[\begin{array}{ccc}
\mathbf{C} & \mathbf{L} & \mathbf{0} \\
\mathbf{0} & \mathbf{0} & \mathbf{0} \\
\mathbf{0} & \mathbf{0} & \mathbf{0}
\end{array}\right]
$$

where $\mathbf{w}_{1}=\left[\hat{\mathbf{u}}^{\mathrm{T}}, \boldsymbol{\psi}^{\mathrm{T}}, \boldsymbol{\lambda}_{1}^{\mathrm{T}}\right]^{\mathrm{T}}$ and $\mathbf{w}_{2}=\left[\partial \hat{\mathbf{u}}^{\mathrm{T}} / \partial z, \partial \boldsymbol{\psi}^{\mathrm{T}} / \partial z, \boldsymbol{\lambda}_{2}^{\mathrm{T}}\right]^{\mathrm{T}}$, are the solution vectors, in which $\boldsymbol{\lambda}_{1}$ and $\boldsymbol{\lambda}_{2}$ are Langrange multipliers that relate to the constraint equations, Eq. (3.51). In addition, the force vectors are formulated as $\mathbf{f}_{1}=\left[\mathbf{0}^{\mathrm{T}}, \boldsymbol{\theta}^{\mathrm{T}}, \mathbf{0}^{\mathrm{T}}\right]^{\mathrm{T}}$ and $\mathbf{f}_{2}=\left[\mathbf{0}^{\mathrm{T}},\left(\mathbf{T}_{r}^{\mathrm{T}} \boldsymbol{\theta}\right)^{\mathrm{T}}, \mathbf{0}^{\mathrm{T}}\right]^{\mathrm{T}}$, the matrices $\mathbf{A}, \mathbf{R}, \mathbf{E}, \mathbf{C}, \mathbf{L}$ can be calculated from Eq. (3.46), and the matrix $\mathbf{D}$ is presented by Eq. 3.52.

For computational convenience, a $6 \times 6$ matrix $\mathbf{W}$ is constructed by column-wise assembling the solutions of Eq. 3.53 for 6 different right-hand sides, which are distinguished by successively setting one specific entry of $\boldsymbol{\theta}$ to unity and the remaining 5 entries to zero. Accordingly, for an arbitrary force vector $\boldsymbol{\theta}$, with the definition of $\boldsymbol{\theta}$ given below Eq. (3.31), the solution vector $\mathbf{w}=\left[\mathbf{w}_{1}, \mathbf{w}_{2}\right]^{\mathrm{T}}$ can be straightforwardly computed as

$$
\mathbf{w}=\mathbf{W} \boldsymbol{\theta} .
$$

In addition, by equating the external complementary virtual work to the internal complementary virtual work, the compliance matrix $\mathbf{F}_{\mathrm{s}}$ of the cross-section follows as 117

$$
\mathbf{F}_{\mathrm{s}}=\mathbf{W}^{\mathrm{T}}\left[\begin{array}{cc}
\mathbf{G}_{11} & \mathbf{H}^{\mathrm{T}} \\
\mathbf{H} & \mathbf{G}_{22}
\end{array}\right] \mathbf{W}=\mathbf{W}^{\mathrm{T}} \mathbf{G} \mathbf{W}
$$

with

$$
\mathbf{G}_{11}=\left[\begin{array}{ccc}
\mathbf{E} & \mathbf{R} & \mathbf{0} \\
\mathbf{R}^{\mathrm{T}} & \mathbf{A} & \mathbf{0} \\
\mathbf{0} & \mathbf{0} & \mathbf{0}
\end{array}\right] \text { and } \mathbf{G}_{22}=\left[\begin{array}{ccc}
\mathbf{M} & \mathbf{0} & \mathbf{0} \\
\mathbf{0} & \mathbf{0} & \mathbf{0} \\
\mathbf{0} & \mathbf{0} & \mathbf{0}
\end{array}\right]
$$

where the matrices $\mathbf{A}, \mathbf{R}, \mathbf{E}, \mathbf{M}$ are provided by Eq. 3.46 , and the matrix $\mathbf{H}$ is given by Eq. (3.54). Finally, the stiffness matrix of the element cross-section used in Eq. (3.31) can be calculated via $\mathbf{K}^{\mathrm{s}}=\mathbf{F}_{\mathrm{s}}^{-1}$, with $\mathbf{F}_{\mathrm{s}}$ in accordance with Eq. 3.56. 


\subsection{Coupled optimization model}

This section presents a coupled optimization model that is able to simultaneously optimize the outer shape and the topological layout of a beam structure. For this purpose, the formulation proposed in [112] is followed, which incorporates the shaperelated design variables in the SIMP topology optimization method. Accordingly, the minimization of the compliance of a structure under a predefined material volume constraint is formulated as

$$
\begin{array}{rll}
\min _{\boldsymbol{\rho}, \mathbf{a}} & c(\boldsymbol{\rho}, \mathbf{a})=\mathbf{f}^{\mathrm{T}} \mathbf{u}(\boldsymbol{\rho}, \mathbf{a}), & \\
\text { subject to } & \frac{V(\boldsymbol{\rho}, \mathbf{a})}{V_{0}}=f_{r}, & \\
\text { with } & l_{s} \leq a_{s} \leq u_{s}, & s=1, \ldots, S, \\
& 0 \leq \rho_{\min } \leq \rho_{e} \leq 1, & e=1, \ldots, N,
\end{array}
$$

in which the structural compliance $c$ depends on both the shape design variables a (i.e., the spatial control points of the NURBS) and the topology design variables $\boldsymbol{\rho}$ (i.e., the relative densities). Further, the variables $l_{s}$ and $u_{s}$ are the lower and upper bounds of the shape design variable $a_{s}, S$ is the total number of shape design variables, $\rho_{\text {min }}$ is the minimum density, $e$ is the element number and $N$ is the total number of elements. The material volume $V$ is a function of both a and $\boldsymbol{\rho}$, and the parameter $V_{0}$ represents the initial volume of the design domain. The variable $f_{r}$ is the prescribed volume fraction, and $\mathbf{f}$ is the global force vector. The displacement vector $\mathbf{u}$ of the modeled system is obtained by solving the corresponding equilibrium equations, i.e., Eq. 3.28 for a 3D beam model and Eq. 3.32 for a 2.5D beam model.

The coupled shape and topology optimization problem for the beam structure is solved in an incremental-iterative fashion using a staggered (sequential) solution strategy, which is illustrated in Figure 3.3

1. The geometry of the design domain is parametrically described by NURBS, as introduced in Section 3.2 Subsequently, for performing a structural analysis, the geometry data is discretized into a FEM model, as described in Section 3.3 .

2. By keeping the shape design variables a momentarily fixed, the problem formulated in Eq. 3.58 can be regarded as a classical topology optimization problem, formulated as

$$
\begin{aligned}
\min _{\boldsymbol{\rho}} & c(\boldsymbol{\rho})=\mathbf{f}^{\mathrm{T}} \mathbf{u}(\boldsymbol{\rho}), \\
\text { subject to } & \frac{V(\boldsymbol{\rho})}{V_{0}}=f_{r}, \\
\text { with } & 0<\rho_{\min } \leq \rho_{e} \leq 1, \quad e=1, \ldots, N .
\end{aligned}
$$

Accordingly, the relative densities $\boldsymbol{\rho}$ are optimized by solving Eq. 3.59, which is carried out by following the typical topology optimization procedure, consisting 


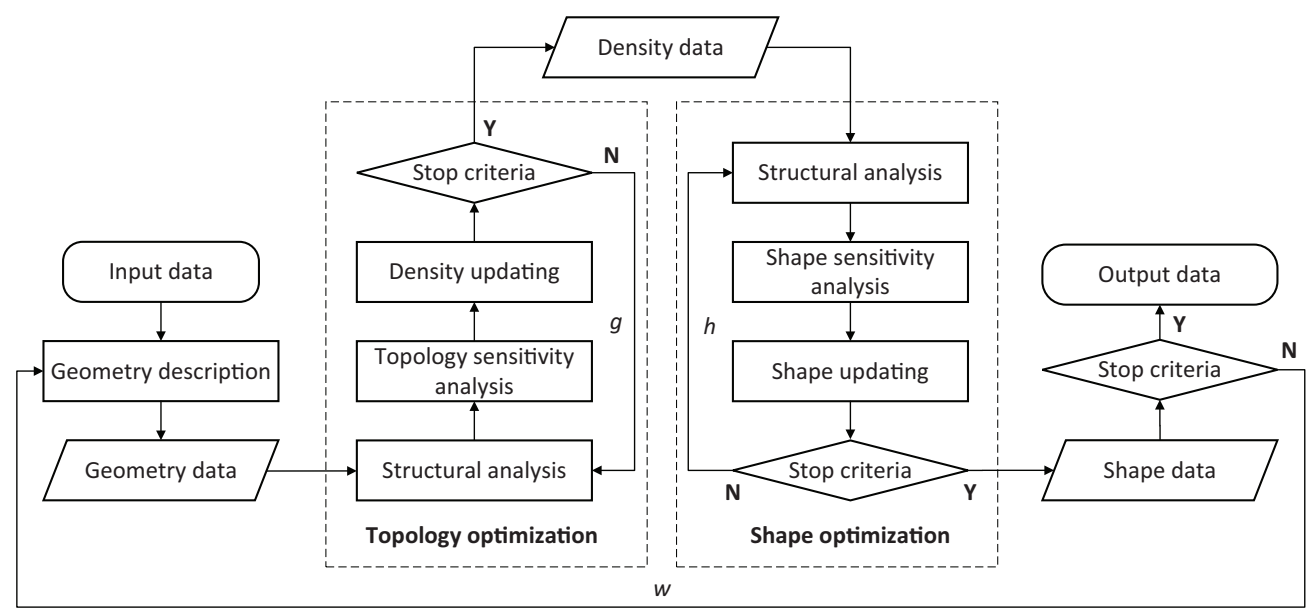

Figure 3.3: Flowchart of the incremental-iterative solution strategy for coupled shape and topology optimization, with iterations $g$ and $h$ referring to topology and shape optimization sub-loops, respectively, and iteration $w$ referring to the outer loop.

of a structural analysis, a topology sensitivity analysis, and the update of the densities.

3. By momentarily freezing the updated densities $\boldsymbol{\rho}$, Eq. 3.58 reduces to a shape optimization problem, formulated as

$$
\begin{array}{rl}
\min _{\mathbf{a}} & c(\mathbf{a})=\mathbf{f}^{\mathrm{T}} \mathbf{u}(\mathbf{a}), \\
\text { subject to } & \frac{V(\mathbf{a})}{V_{0}}=f_{r}, \\
\text { with } & l_{s} \leq a_{s} \leq u_{s}, \quad s=1, \ldots, S .
\end{array}
$$

The shape design variables a are calculated by solving Eq. 3.60 based on the densities $\boldsymbol{\rho}$ found in step 2. Step 3 is carried out by performing a standard gradient-based shape optimization procedure, consisting of a structural analysis, a shape sensitivity analysis, and the updating of the shape.

4. When the element densities $\boldsymbol{\rho}$ obtained from step 2 are not optimal for the shape subsequently computed in step 3 , in a sense that they do not lead to an immediate satisfaction of the shape convergence criteria (i.e., after the first iteration), a new topology optimization increment (step 2) is performed, based on the updated shape design variables a. After this step has converged and the topology design variables $\boldsymbol{\rho}$ have been updated, a new shape optimization increment (step 3 ) is performed, and the above check on immediate convergence is repeated. The above process is continued until the incremental-iterative solution procedure has converged. Note that updating the topology and shape configurations of the beam after convergence of the corresponding optimization increment causes the coupling effects between topology and shape to be automatically accounted for. 


\subsection{Sensitivity analysis}

The convergence (or stop) criteria for the individual topology and shape optimization increments and for the overall procedure are, respectively, evaluated during every iteration $g, h$ and $w$, see Figure 3.3, and will be specified when discussing the numerical examples in Section 3.6

The staggered solution strategy depicted in Figure 3.3 starts with an incremental topology optimization step, and, as such, is referred to as Coupled Topology Shape Optimization (CTSO). Alternatively, it can start with an incremental shape optimization step, and will then be referred to as Coupled Shape Topology Optimization (CSTO), see also [112. In shape and topology optimization problems the landscape of solutions is characterized by numerous local minima, whereby the convergence speed and the specific optimized solution calculated may be expected to be sensitive to the algorithmic features of the numerical update scheme applied. Despite that the main focus of the present work is on numerical discretization aspects and on comparing the outer and internal geometries of the 3D and 2.5D beam models under shape and topology optimization, possible differences in the results from the CTSO and CSTO update schemes will be also analyzed and discussed. Note that the choice of a staggered update scheme in this study is arbitrary; the coupled optimization problem, Eq. (3.58), could also be solved concurrently by using a monolithic scheme. A systematic comparison of the influence of staggered and monolithic update schemes on the computational efficiency and outcome of the coupled optimization approach refers to 120.

\subsection{Sensitivity analysis}

For the coupled, gradient-based optimization approach, the analytical sensitivities of the structural compliance $c$ need to be determined with respect to the shape design variables a and relative element densities $\boldsymbol{\rho}$. These analytical sensitivities are elaborated below for the $2.5 \mathrm{D}$ and $3 \mathrm{D}$ beam models.

\subsection{D beam model}

For simplicity of notation, the variable $d$ is introduced, which represents either the shape design variable $a_{s}$ or the element density $\rho_{e}$. In correspondence with Eq. (3.56), the derivative of the cross-sectional compliance matrix $\mathbf{F}_{\mathrm{s}}$ with respect to $d$ can be expressed as

$$
\frac{\partial \mathbf{F}_{\mathrm{s}}}{\partial d}=\frac{\partial \mathbf{W}^{\mathrm{T}}}{\partial d} \mathbf{G} \mathbf{W}+\mathbf{W}^{\mathrm{T}} \frac{\partial \mathbf{G}}{\partial d} \mathbf{W}+\mathbf{W}^{\mathrm{T}} \mathbf{G} \frac{\partial \mathbf{W}}{\partial d}
$$

Since the matrix $\mathbf{W}$ contains the solutions of the equilibrium equations given by Eq. (3.53), whereby in $\hat{\mathbf{f}}$ one component of $\boldsymbol{\theta}$ equals unity and the remaining components are zero, the derivative $\partial \mathbf{W} / \partial d$ in Eq. (3.61) follows from Eq. (3.53) as

$$
\frac{\partial \mathbf{W}}{\partial d}=-\hat{\mathbf{K}}^{-1} \frac{\partial \hat{\mathbf{K}}}{\partial d} \mathbf{W}
$$


Inserting Eq. 3.62 into Eq. (3.61), the derivative of the cross-sectional compliance matrix becomes

$$
\frac{\partial \mathbf{F}_{\mathrm{s}}}{\partial d}=-\mathbf{W}^{\mathrm{T}} \frac{\partial \hat{\mathbf{K}}^{\mathrm{T}}}{\partial d} \mathbf{V}+\mathbf{W}^{\mathrm{T}} \frac{\partial \mathbf{G}}{\partial d} \mathbf{W}-\mathbf{V}^{\mathrm{T}} \frac{\partial \hat{\mathbf{K}}}{\partial d} \mathbf{W},
$$

with

$$
\mathbf{V}=\hat{\mathbf{K}}^{-\mathrm{T}} \mathbf{G W}
$$

where the matrix $\hat{\mathbf{K}}$ is obtained from Eqs. 3.53 and 3.54 , and $\mathbf{G}$ follows from Eqs. (3.56) and (3.57). Since the stiffness matrix $\mathbf{K}^{\mathbf{s}}$ of the cross-section is the inverse of the compliance matrix $\mathbf{F}_{\mathrm{s}}$, the derivative of the stiffness matrix with respect to $d$ may be expressed as

$$
\frac{\partial \mathbf{K}^{\mathrm{s}}}{\partial d}=\frac{\partial \mathbf{F}_{\mathrm{s}}^{-1}}{\partial d}=-\mathbf{F}_{\mathrm{s}}^{-1} \frac{\partial \mathbf{F}_{\mathrm{s}}}{\partial d} \mathbf{F}_{\mathrm{s}}^{-1}=-\mathbf{K}^{\mathrm{s}} \frac{\partial \mathbf{F}_{\mathrm{s}}}{\partial d} \mathbf{K}^{\mathrm{s}}
$$

The global stiffness matrix $\overline{\mathbf{K}}$ of the beam model is based on the cross-sectional stiffness matrix $\mathbf{K}_{e}^{\mathrm{s}}$ of the beam element via Eqs. (3.33) and (3.34). Accordingly, the derivative of $\overline{\mathbf{K}}$ with respect to the design variable $d$ becomes

$$
\frac{\partial \overline{\mathbf{K}}}{\partial d}=\sum_{e=1}^{N} \int_{-1}^{1}\left(\overline{\mathbf{B}}_{e} \mathbf{N}_{e}\right)^{\mathrm{T}} \frac{\partial \mathbf{K}_{e}^{\mathrm{s}}}{\partial d}\left|\overline{\mathbf{J}}_{e}\right| \overline{\mathbf{B}}_{e} \mathbf{N}_{e} d \zeta_{e} .
$$

Because the loads $\mathbf{f}$ are independent of the design variable $d$, from Eqs. (3.58), 3.32 and the symmetry relation $\overline{\mathbf{K}}=\overline{\mathbf{K}}^{T}$, the sensitivity of the compliance $c$ with respect to $d$ follows as

$$
\frac{\partial c}{\partial d}=-\overline{\mathbf{u}}^{\mathrm{T}} \frac{\partial \overline{\mathbf{K}}}{\partial d} \overline{\mathbf{u}}
$$

The specific forms of the derivatives $\partial \hat{\mathbf{K}} / \partial d$ and $\partial \mathbf{G} / \partial d$ appearing in the above equations depend on the type of design variable. The derivation of these terms is presented below.

\subsubsection{Shape sensitivity analysis}

The shape sensitivity analysis starts with the computation of the partial derivative of the Jacobian matrix, Eq. 3.48, with respect to the shape design variable $a_{s}$, leading to

$$
\partial_{s}\left(\hat{\mathbf{J}}_{e}\right)=\partial_{s}\left(\overline{\mathbf{P}}_{e}\right)\left(\hat{\boldsymbol{\partial}} \mathbf{N}_{e}^{\mathrm{T}}\right)
$$


in which, for reasons of brevity, the notation $\partial_{s}(\cdot)=\partial(\cdot) / \partial a_{s}$ has been used. The derivative $\partial_{s}\left(\overline{\mathbf{P}}_{e}\right)$ appearing in Eq. (3.68) is calculated by combining Eqs. (3.21) and (3.16), in which the derivative of the coordinates of an arbitrary point on the NURBS surface with respect to the shape design variable follows from Eq. (3.11) as

$$
\partial_{s}\left(\mathbf{p}^{\mathrm{T}}\right)=\left[\frac{\mathbf{N}_{\mathrm{b}}^{\mathrm{T}} \partial_{s}\left(\mathbf{P}_{k, X}^{2 \mathrm{D}}\right) \mathbf{M}_{\mathrm{b}}}{\mathbf{N}_{\mathrm{b}}^{\mathrm{T}} \boldsymbol{\Omega}_{k}^{2 \mathrm{D}} \mathbf{M}_{\mathrm{b}}}, \frac{\mathbf{N}_{\mathrm{b}}^{\mathrm{T}} \partial_{s}\left(\mathbf{P}_{k, Y}^{2 \mathrm{D}}\right) \mathbf{M}_{\mathrm{b}}}{\mathbf{N}_{\mathrm{b}}^{\mathrm{T}} \boldsymbol{\Omega}_{k}^{2 \mathrm{D}} \mathbf{M}_{\mathrm{b}}}, \frac{\mathbf{N}_{\mathrm{b}}^{\mathrm{T}} \partial_{s}\left(\mathbf{P}_{k, Z}^{2 \mathrm{D}}\right) \mathbf{M}_{\mathrm{b}}}{\mathbf{N}_{\mathrm{b}}^{\mathrm{T}} \mathbf{\Omega}_{k}^{2 \mathrm{D}} \mathbf{M}_{\mathrm{b}}}\right],
$$

where $\partial_{s}\left(\mathbf{P}_{k, C}^{2 \mathrm{D}}\right)$, with $C \in\{X, Y, Z\}$, can be easily determined from Eq. 3.10 , in which the $z$-coordinate of the control points $Z_{i, j, k}$ is set to zero. Further, the matrices $\mathbf{N}_{\mathrm{b}}, \mathbf{M}_{\mathrm{b}}$ and $\boldsymbol{\Omega}_{k}^{2 \mathrm{D}}$ are obtained from Eqs. (3.8), (3.9) and 3.12 , respectively. Eq. (3.68) can be used for the calculation of the derivative of the determinant of the Jacobian with respect to the shape design variable $a_{s}$ via

$$
\partial_{s}\left(\left|\hat{\mathbf{J}}_{e}\right|\right)=\left(\partial_{s}\left(\hat{\mathbf{J}}_{e}\right)\right)_{11}\left(\hat{\mathbf{J}}_{e}\right)_{22}+\left(\hat{\mathbf{J}}_{e}\right)_{11}\left(\partial_{s}\left(\hat{\mathbf{J}}_{e}\right)\right)_{22}-\left(\partial_{s}\left(\hat{\mathbf{J}}_{e}\right)\right)_{12}\left(\hat{\mathbf{J}}_{e}\right)_{21}-\left(\hat{\mathbf{J}}_{e}\right)_{12}\left(\partial_{s}\left(\hat{\mathbf{J}}_{e}\right)\right)_{21},
$$

Further, Eq. 3.68 can be employed in the expression for the derivative of the inverse of the Jacobian matrix with respect to the shape design variable $a_{s}$, which reads:

$$
\partial_{s}\left(\hat{\mathbf{J}}_{e}^{-1}\right)=-\hat{\mathbf{J}}_{e}^{-1} \partial_{s}\left(\hat{\mathbf{J}}_{e}\right) \hat{\mathbf{J}}_{e}^{-1}
$$

with $\hat{\mathbf{J}}_{e}$ provided by Eq. 3.48 .

With the operator $\hat{\mathbf{B}}_{e}$ given by Eq. 3.49, the matrix product $\hat{\mathbf{B}}_{e} \mathbf{N}_{e}$ in Eq. 3.46 can be expressed as

$$
\hat{\mathbf{B}}_{e} \mathbf{N}_{e}=\hat{\mathbf{B}}_{e}^{1} \frac{\partial \mathbf{N}_{e}}{\partial \xi_{e}}+\hat{\mathbf{B}}_{e}^{2} \frac{\partial \mathbf{N}_{e}}{\partial \eta_{e}}
$$

where $\hat{\mathbf{B}}_{e}^{c}$, with $c \in\{1,2\}$, is obtained from Eq. 3.50. Accordingly, the derivative of $\hat{\mathbf{B}}_{e} \mathbf{N}_{e}$ with respect to the shape design variable $a_{s}$ can be formulated as

$$
\partial_{s}\left(\hat{\mathbf{B}}_{e} \mathbf{N}_{e}\right)=\partial_{s}\left(\hat{\mathbf{B}}_{e}^{1}\right) \frac{\partial \mathbf{N}_{e}}{\partial \xi_{e}}+\partial_{s}\left(\hat{\mathbf{B}}_{e}^{2}\right) \frac{\partial \mathbf{N}_{e}}{\partial \eta_{e}}
$$

with

$$
\partial_{s}\left(\hat{\mathbf{B}}_{e}^{c}\right)=\left[\begin{array}{ccc}
\left(\partial_{s}\left(\hat{\mathbf{J}}_{e}^{-1}\right)\right)_{1 c} & 0 & 0 \\
0 & \left(\partial_{s}\left(\hat{\mathbf{J}}_{e}^{-1}\right)\right)_{2 c} & 0 \\
\left(\partial_{s}\left(\hat{\mathbf{J}}_{e}^{-1}\right)\right)_{2 c} & \left(\partial_{s}\left(\hat{\mathbf{J}}_{e}^{-1}\right)\right)_{1 c} & 0 \\
0 & 0 & \left(\partial_{s}\left(\hat{\mathbf{J}}_{e}^{-1}\right)\right)_{1 c} \\
0 & 0 & \left(\partial_{s}\left(\hat{\mathbf{J}}_{e}^{-1}\right)\right)_{2 c} \\
0 & 0 & 0
\end{array}\right]
$$


in which $c \in\{1,2\}$, and $\partial_{s}\left(\hat{\mathbf{J}}_{e}^{-1}\right)$ is given by Eq. 3.71). The derivatives of the coefficient matrices $\mathbf{E}, \mathbf{C}$, and $\mathbf{M}$ - presented in Eq. $(3.46)$ - with respect to the shape design variable $a_{s}$ read

$$
\begin{aligned}
& \partial_{s}(\mathbf{E})=\sum_{e=1}^{N} \int_{-1}^{1} \int_{-1}^{1}\left[\begin{array}{l}
\left.\partial_{s}\left(\left(\hat{\mathbf{B}}_{e} \mathbf{N}_{e}\right)^{\mathrm{T}}\right) \mathbf{Q}_{e} \hat{\mathbf{B}}_{e} \mathbf{N}_{e}\left|\hat{\mathbf{J}}_{e}\right|+\left(\hat{\mathbf{B}}_{e} \mathbf{N}_{e}\right)^{\mathrm{T}} \mathbf{Q}_{e} \partial_{s}\left(\hat{\mathbf{B}}_{e} \mathbf{N}_{e}\right)\left|\hat{\mathbf{J}}_{e}\right|\right] d \xi_{e} d \eta_{e}, \\
+\left(\hat{\mathbf{B}}_{e} \mathbf{N}_{e}\right)^{\mathrm{T}} \mathbf{Q}_{e} \hat{\mathbf{B}}_{e} \mathbf{N}_{e} \partial_{s}\left(\left|\hat{\mathbf{J}}_{e}\right|\right)
\end{array}\right. \\
& \partial_{s}(\mathbf{C})=\sum_{e=1}^{N} \int_{-1}^{1} \int_{-1}^{1}\left[\left(\mathbf{S}_{e} \mathbf{N}_{e}\right)^{\mathrm{T}} \mathbf{Q}_{e} \partial_{s}\left(\hat{\mathbf{B}}_{e} \mathbf{N}_{e}\right)\left|\hat{\mathbf{J}}_{e}\right|+\left(\mathbf{S}_{e} \mathbf{N}_{e}\right)^{\mathrm{T}} \mathbf{Q}_{e} \hat{\mathbf{B}}_{e} \mathbf{N}_{e} \partial_{s}\left(\left|\hat{\mathbf{J}}_{e}\right|\right)\right] d \xi_{e} d \eta_{e}, \\
& \partial_{s}(\mathbf{M})=\sum_{e=1}^{N} \int_{-1}^{1} \int_{-1}^{1}\left[\left(\mathbf{S}_{e} \mathbf{N}_{e}\right)^{\mathrm{T}} \mathbf{Q}_{e} \mathbf{S}_{e} \mathbf{N}_{e} \partial_{s}\left(\left|\hat{\mathbf{J}}_{e}\right|\right)\right] d \xi_{e} d \eta_{e} .
\end{aligned}
$$

From the last two expressions in Eq. (3.75), the derivatives with respect to the shape design variable of the matrices $\mathbf{A}, \mathbf{R}$ and $\mathbf{L}$ in Eq. (3.46) are calculated as

$$
\partial_{s}(\mathbf{A})=\mathbf{D}^{\mathrm{T}} \partial_{s}(\mathbf{M}) \mathbf{D}, \partial_{s}(\mathbf{R})=\left(\partial_{s}(\mathbf{C})\right)^{\mathrm{T}} \mathbf{D}, \partial_{s}(\mathbf{L})=\partial_{s}(\mathbf{M}) \mathbf{D}
$$

with $\mathbf{D}$ given by Eq. 3.52. Finally, the terms $\partial \hat{\mathbf{K}} / \partial a_{s}$ and $\partial \mathbf{G} / \partial a_{s}$ in Eq. 3.63 are calculated from Eqs. (3.53) to (3.57), thereby inserting the derivatives of the coefficient matrices given by Eqs. (3.75) and 3.76).

\subsubsection{Topology sensitivity analysis}

According to Eqs. (3.26) and (3.46), the coefficient matrices A, R, E, C, L and $\mathbf{M}$ of the cross-section can be explicitly expressed as a function of the element density $\rho_{e}$. Correspondingly, by referring to an arbitrary coefficient matrix as $\mathbf{M}^{\mathbf{c}}$, its derivative with respect to the element density is calculated as

$$
\frac{\partial \mathbf{M}^{\mathrm{c}}}{\partial \rho_{e}}=p\left(\rho_{e}\right)^{p-1} \mathbf{M}_{0}^{\mathrm{c}},
$$

where the variable $\mathbf{M}_{0}^{\mathrm{c}}$ refers to the corresponding coefficient matrices obtained from Eq. (3.46) by substituting the density-independent constitutive matrix $\mathbf{Q}$ (instead of the density-dependent constitutive matrix $\mathbf{Q}_{e}$, see Eq. (3.26). With Eq. (3.77), the terms $\partial \hat{\mathbf{K}} / \partial \rho_{e}$ and $\partial \mathbf{G} / \partial \rho_{e}$ in Eq. 3.63) can be determined from Eqs. (3.53) to (3.57). To alleviate mesh dependency and possible checkerboard patterns in the solution computed by topology optimization, a sensitivity filter [66] is introduced that smoothens the sensitivity of the structural compliance as follows

$$
\frac{\partial \hat{c}}{\partial \rho_{e}}=\frac{1}{\rho_{e} \sum_{f=1}^{N} \hat{H}_{f}} \sum_{f=1}^{N} \hat{H}_{f} \rho_{f} \frac{\partial c}{\partial \rho_{f}},
$$


where $\partial c / \partial \rho_{f}$ is calculated employing Eqs. 3.63 to 3.67$)$. Further, $\hat{H}_{f}=\max \left(0, r_{\min }-\right.$ $\operatorname{dist}(e, f))$, in which $\operatorname{dist}(e, f)$ is the distance between the center of element $e$ and the center of element $f$, and $r_{\text {min }}$ is the radius of the circle within which smoothing takes place.

\subsubsection{D beam model}

\subsubsection{Shape sensitivity analysis}

In the three-dimensional FEM model for the beam structure, the Jacobian matrix of an element $e$ is presented by Eq. (3.20). Correspondingly, its derivative with respect to the shape design variable $a_{s}$ follows from

$$
\partial_{s}\left(\mathbf{J}_{e}\right)=\partial_{s}\left(\overline{\mathbf{P}}_{e}\right)\left(\boldsymbol{\partial \mathbf { N } _ { e } ^ { \mathrm { T } }}\right) .
$$

The term $\partial_{s}\left(\overline{\mathbf{P}}_{e}\right)$ in Eq. 3.79$)$ is calculated by combining Eqs. 3.21) and 3.16) with the derivative of the nodal coordinates, Eq. 3.14, with respect to $a_{s}$ :

$$
\partial_{s}\left(\mathbf{p}^{\mathrm{T}}\right)=\left[\frac{\mathbf{L}_{\mathrm{b}}^{\mathrm{T}} \partial_{s}\left(\mathbf{P}_{X}^{3 \mathrm{D}}\right)}{\mathbf{L}_{\mathrm{b}}^{\mathrm{T}} \boldsymbol{\Omega}^{3 \mathrm{D}}}, \frac{\mathbf{L}_{\mathrm{b}}^{\mathrm{T}} \partial_{s}\left(\mathbf{P}_{Y}^{3 \mathrm{D}}\right)}{\mathbf{L}_{\mathrm{b}}^{\mathrm{T}} \boldsymbol{\Omega}^{3 \mathrm{D}}}, \frac{\mathbf{L}_{\mathrm{b}}^{\mathrm{T}} \partial_{s}\left(\mathbf{P}_{Z}^{3 \mathrm{D}}\right)}{\mathbf{L}_{\mathrm{b}}^{\mathrm{T}} \boldsymbol{\Omega}^{3 \mathrm{D}}}\right],
$$

with

$$
\partial_{s}\left(\mathbf{P}_{C}^{3 \mathrm{D}}\right)=\left[\mathbf{N}_{\mathrm{b}}^{\mathrm{T}} \partial_{s}\left(\mathbf{P}_{1, C}^{2 \mathrm{D}}\right) \mathbf{M}_{\mathrm{b}}, \mathbf{N}_{\mathrm{b}}^{\mathrm{T}} \partial_{s}\left(\mathbf{P}_{2, C}^{2 \mathrm{D}}\right) \mathbf{M}_{\mathrm{b}}, \cdots, \mathbf{N}_{\mathrm{b}}^{\mathrm{T}} \partial_{s}\left(\mathbf{P}_{l, C}^{2 \mathrm{D}}\right) \mathbf{M}_{\mathrm{b}}\right]^{\mathrm{T}},
$$

where $\partial_{s}\left(\mathbf{P}_{k, C}^{2 \mathrm{D}}\right)$, with $C \in\{X, Y, Z\}$ and $k=1,2, \cdots, l$, is determined based on Eq. 3.10 , and the matrices $\mathbf{L}_{\mathrm{b}}, \mathbf{N}_{\mathrm{b}}, \mathbf{M}_{\mathrm{b}}$ and $\boldsymbol{\Omega}_{k}^{3 \mathrm{D}}$ are obtained from Eqs. 3.3), 3.8), (3.9) and 3.15), respectively. From Eq. 3.79), the derivative of the determinant of the Jacobian matrix with respect to $a_{s}$ can be computed via

$$
\begin{aligned}
\partial_{s}\left(|\mathbf{J}|_{e}\right)= & \left.\left(\partial_{s}\left(\mathbf{J}_{e}\right)\right)_{11}\left[\left(\mathbf{J}_{e}\right)_{22}\left(\mathbf{J}_{e}\right)_{33}-\left(\mathbf{J}_{e}\right)_{23}\left(\mathbf{J}_{e}\right)_{32}\right)\right] \\
& +\left(\mathbf{J}_{e}\right)_{11}\left[\begin{array}{l}
\left(\partial_{s}\left(\mathbf{J}_{e}\right)\right)_{22}\left(\mathbf{J}_{e}\right)_{33}+\left(\mathbf{J}_{e}\right)_{22}\left(\partial_{s}\left(\mathbf{J}_{e}\right)\right)_{33} \\
-\left(\partial_{s}\left(\mathbf{J}_{e}\right)\right)_{23}\left(\mathbf{J}_{e}\right)_{32}-\left(\mathbf{J}_{e}\right)_{23}\left(\partial_{s}\left(\mathbf{J}_{e}\right)\right)_{32}
\end{array}\right] \\
& +\left(\partial_{s}\left(\mathbf{J}_{e}\right)\right)_{21}\left[\left(\mathbf{J}_{e}\right)_{13}\left(\mathbf{J}_{e}\right)_{32}-\left(\mathbf{J}_{e}\right)_{12}\left(\mathbf{J}_{e}\right)_{33}\right] \\
& +\left(\mathbf{J}_{e}\right)_{21}\left[\begin{array}{l}
\left(\partial_{s}\left(\mathbf{J}_{e}\right)\right)_{13}\left(\mathbf{J}_{e}\right)_{32}+\left(\mathbf{J}_{e}\right)_{13}\left(\partial_{s}\left(\mathbf{J}_{e}\right)\right)_{32} \\
-\left(\partial_{s}\left(\mathbf{J}_{e}\right)\right)_{12}\left(\mathbf{J}_{e}\right)_{33}-\left(\mathbf{J}_{e}\right)_{12}\left(\partial_{s}\left(\mathbf{J}_{e}\right)\right)_{33}
\end{array}\right] \\
& +\left(\partial_{s}\left(\mathbf{J}_{e}\right)\right)_{31}\left[\left(\mathbf{J}_{e}\right)_{12}\left(\mathbf{J}_{e}\right)_{23}-\left(\mathbf{J}_{e}\right)_{13}\left(\mathbf{J}_{e}\right)_{22}\right] \\
& +\left(\mathbf{J}_{e}\right)_{31}\left[\begin{array}{l}
\left(\partial_{s}\left(\mathbf{J}_{e}\right)\right)_{12}\left(\mathbf{J}_{e}\right)_{23}+\left(\mathbf{J}_{e}\right)_{12}\left(\partial_{s}\left(\mathbf{J}_{e}\right)\right)_{23} \\
-\left(\partial_{s}\left(\mathbf{J}_{e}\right)\right)_{13}\left(\mathbf{J}_{e}\right)_{22}-\left(\mathbf{J}_{e}\right)_{13}\left(\partial_{s}\left(\mathbf{J}_{e}\right)\right)_{22}
\end{array}\right],
\end{aligned}
$$

while the derivative of the inverse of the Jacobian matrix becomes 


$$
\partial_{s}\left(\mathbf{J}_{e}^{-1}\right)=-\mathbf{J}_{e}^{-1} \partial_{s}\left(\mathbf{J}_{e}\right) \mathbf{J}_{e}^{-1},
$$

with the Jacobian matrix $\mathbf{J}_{e}$ given by Eq. 3.20 . In addition, the derivative of the matrix product $\mathbf{B}_{e} \mathbf{N}_{e}$ used in Eq. 3.25) is calculated with the use of Eqs. 3.23) and (3.24) as

$$
\partial_{s}\left(\mathbf{B}_{e} \mathbf{N}_{e}\right)=\partial_{s}\left(\mathbf{B}_{e}^{1}\right) \frac{\partial \mathbf{N}_{e}}{\partial \xi_{e}}+\partial_{s}\left(\mathbf{B}_{e}^{2}\right) \frac{\partial \mathbf{N}_{e}}{\partial \eta_{e}}+\partial_{s}\left(\mathbf{B}_{e}^{3}\right) \frac{\partial \mathbf{N}_{e}}{\partial \zeta_{e}},
$$

with

$$
\partial_{s}\left(\mathbf{B}_{e}^{c}\right)=\left[\begin{array}{ccc}
\left(\partial_{s}\left(\mathbf{J}_{e}^{-1}\right)\right)_{1 c} & 0 & 0 \\
0 & \left(\partial_{s}\left(\mathbf{J}_{e}^{-1}\right)\right)_{2 c} & 0 \\
\left(\partial_{s}\left(\mathbf{J}_{e}^{-1}\right)\right)_{2 c} & \left(\partial_{s}\left(\mathbf{J}_{e}^{-1}\right)\right)_{1 c} & 0 \\
\left(\partial_{s}\left(\mathbf{J}_{e}^{-1}\right)\right)_{3 c} & 0 & \left(\partial_{s}\left(\mathbf{J}_{e}^{-1}\right)\right)_{1 c} \\
0 & \left(\partial_{s}\left(\mathbf{J}_{e}^{-1}\right)\right)_{3 c} & \left(\partial_{s}\left(\mathbf{J}_{e}^{-1}\right)\right)_{2 c} \\
0 & 0 & \left(\partial_{s}\left(\mathbf{J}_{e}^{-1}\right)\right)_{3 c}
\end{array}\right],
$$

in which $c \in\{1,2,3\}$ and $\partial_{s}\left(\mathbf{J}_{e}^{-1}\right)$ is computed from Eq. (3.83). With Eq. (3.84) and the global stiffness matrix $\mathbf{K}$ given by Eq. (3.27), the derivative of the stiffness matrix with respect to the shape design variable $a_{s}$ can be computed as

$$
\partial_{s}(\mathbf{K})=\sum_{e=1}^{N} \int_{-1}^{1} \int_{-1}^{1} \int_{-1}^{1}\left[\begin{array}{c}
\partial_{s}\left(\left(\mathbf{B}_{e} \mathbf{N}_{e}\right)^{\mathrm{T}}\right) \mathbf{Q}_{e} \mathbf{B}_{e} \mathbf{N}_{e}\left|\mathbf{J}_{e}\right| \\
+\left(\mathbf{B}_{e} \mathbf{N}_{e}\right)^{\mathrm{T}} \mathbf{Q}_{e} \partial_{s}\left(\mathbf{B}_{e} \mathbf{N}_{e}\right)\left|\mathbf{J}_{e}\right| \\
+\left(\mathbf{B}_{e} \mathbf{N}_{e}\right)^{\mathrm{T}} \mathbf{Q}_{e} \mathbf{B}_{e} \mathbf{N}_{e} \partial_{s}\left(\left|\mathbf{J}_{e}\right|\right)
\end{array}\right] d \xi_{e} d \eta_{e} d \zeta_{e} .
$$

Following the assumption that the load vector $\mathbf{f}$ is independent of the design variables, the derivative of the structural compliance with respect to the shape design variable $a_{s}$ becomes

$$
\partial_{s}(c)=-\mathbf{u}^{\mathrm{T}} \partial_{s}(\mathbf{K}) \mathbf{u}
$$

where $\partial_{s}(\mathbf{K})$ is given by Eq. (3.86) and $\mathbf{u}$ is the global displacement vector obtained from solving the system of equilibrium equations, Eq. (3.28).

\subsubsection{Topology sensitivity analysis}

In correspondence with Eqs. (3.28) and (3.58), the derivative of the structural compliance $c$ with respect to the relative density $\rho_{e}$ equals

$$
\frac{\partial c}{\partial \rho_{e}}=-\mathbf{u}^{\mathrm{T}} \frac{\partial \mathbf{K}}{\partial \rho_{e}} \mathbf{u}
$$


Since the three-dimensional element stiffness matrix is an explicit function of the element density $\rho_{e}$, see Eqs. (3.25) and (3.26), the derivative of the element stiffness matrix $\mathbf{k}_{e}$ with respect to $\rho_{e}$ follows as

$$
\frac{\partial \mathbf{k}_{e}}{\partial \rho_{e}}=p\left(\rho_{e}\right)^{p-1} \mathbf{k}_{0},
$$

where $\mathbf{k}_{0}$ is obtained from Eq. 3.25), by substituting the density-independent constitutive matrix $\mathbf{Q}$ (instead of the density-dependent constitutive matrix $\mathbf{Q}_{e}$, see Eq. (3.26)). Correspondingly, the derivative $\partial c / \partial \rho_{e}$ provided by Eq. 3.88 is reformulated for a specific element $e$ using Eqs. 3.89) and (3.27), i.e.,

$$
\frac{\partial c}{\partial \rho_{e}}=-\left(\mathbf{u}_{e}\right)^{\mathrm{T}} \frac{\partial \mathbf{k}_{e}}{\partial \rho_{e}} \mathbf{u}_{e}=-\left(\mathbf{u}_{e}\right)^{\mathrm{T}} p\left(\rho_{e}\right)^{p-1} \mathbf{k}_{0} \mathbf{u}_{e} .
$$

The filtering of Eq. 3.90) is performed in accordance with Eq. (3.78), where $r_{\min }$ is the radius of a sphere (instead of a circle) within which smoothing occurs.

\subsection{Numerical examples}

\subsubsection{Validation case studies}

In order to validate the accuracy and efficiency of the coupled shape and topology optimization procedure for $2.5 \mathrm{D}$ beam models, an elastic cantilever beam is considered whereby the solution obtained for the $2.5 \mathrm{D}$ beam model is compared to that for the $3 \mathrm{D}$ beam model. The initial configuration of the cantilever beam is illustrated in Figure 3.4 showing a slender beam with length $L_{0}$ and a uniform rectangular crosssection with dimensions $W_{0} \times H_{0}$. The left end of the beam is fully clamped (i.e., zero displacements and rotations in all directions), and the right end is subjected to a vertical load $F$. The values of the model parameters are listed in Table 3.1, whereby $E$ and $\nu$ are the Young's modulus and Poisson's ratio of the beam material, respectively.

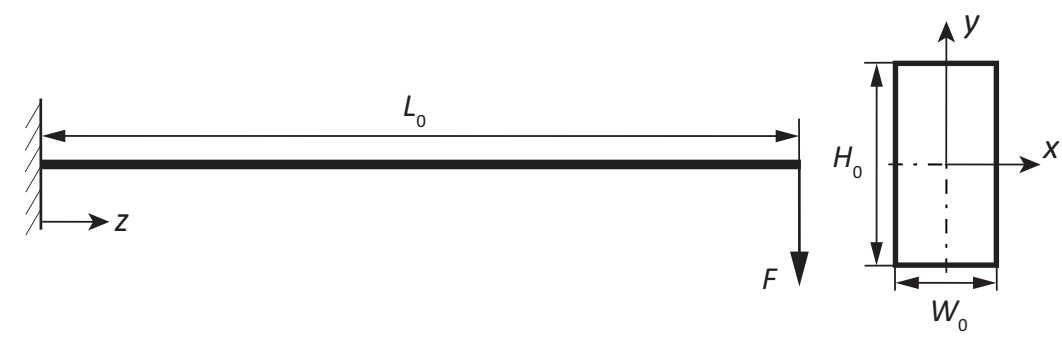

Figure 3.4: Cantilever beam with a uniform rectangular cross-section. 
Table 3.1: Parameter values of the cantilever beam problem.

\begin{tabular}{lllllll}
\hline Parameter & $\begin{array}{l}L_{0} \\
(\mathrm{~m})\end{array}$ & $\begin{array}{l}W_{0} \\
(\mathrm{~m})\end{array}$ & $\begin{array}{l}H_{0} \\
(\mathrm{~m})\end{array}$ & $\begin{array}{l}E \\
(\mathrm{MPa})\end{array}$ & $\begin{array}{l}\nu \\
(-)\end{array}$ & $\begin{array}{l}F \\
(\mathrm{kN})\end{array}$ \\
\hline Value & 30 & 1 & 2 & $210 \times 10^{3}$ & 0.3 & 10 \\
\hline
\end{tabular}

\subsubsection{Shape optimization}

As a first step, the comparison study focuses only on the optimization of the shape of the beam sketched in Figure 3.4 and the topology optimization is temporarily left out of consideration. In the 3D model the shape of the cantilever beam is parametrically described by a NURBS solid. As explained in Section 3.2.3 the NURBS solid is constructed using a one-dimensional NURBS curve in the axial direction of the beam and a two-dimensional NURBS surface for the description of the beam cross-section. According to the definition of a NURBS surface introduced in Section 3.2.2 the polynomial orders in Eq. (3.7) are set to $p=2$ and $q=2$, and the number of basis functions in the $\xi$ - and $\eta$-directions is equal to $n=3$ and $m=3$, respectively, in correspondence with the knot vectors defined as

$$
\boldsymbol{\Xi}=[0,0,0,1,1,1]^{\mathrm{T}}, \boldsymbol{H}=[0,0,0,1,1,1]^{\mathrm{T}} .
$$

Correspondingly, the NURBS surface is characterized by a net of 9 control points, with the arrangement of the control points at the $k$-th location along the axial $(z-)$ direction of the beam illustrated in Figure 3.5. where $\mathbf{C}_{i, j, k}=\left(X_{i, j, k}, Y_{i, j, k}, Z_{i, j, k}\right)$, with $i=1,2,3, j=1,2,3$ and $k=1,2, \cdots, l$, see also Eq. (3.10). The initial coordinates and weights of these control points are listed in Table 3.2 , in which $Z_{k}$ with $k=1,2, \cdots, l$ is used to replace $Z_{i, j, k}$ since all control points of a specific surface have the same $z$-coordinate. The value of the $z$-coordinate $Z_{k}$ of each NURBS surface is determined based on the NURBS curve, see Section 3.2.1 which describes the geometry along the beam length. The polynomial order of the NURBS curve is $r=1$, which, in correspondence with Eq. 3.1), represents a linear curve. In order to construct the complete beam geometry, $l=81$ NURBS surfaces are equally spaced along the beam length $L_{0}$. Accordingly, the knot vector $\boldsymbol{Z}$ in Eq. (3.1) is defined as

$$
\boldsymbol{Z}=[0, \ldots,(k-1) /(l-1), \ldots, 1]^{\mathrm{T}} \quad \text { with } \quad k=1,2, \cdots, l,
$$

and the $z$-coordinate $Z_{k}$ of the control points is

$$
Z_{k}=\frac{(k-1) L_{0}}{l-1} \quad \text { with } \quad k=1,2, \cdots, l,
$$

with the beam length $L_{0}$ given in Table 3.1

With the above characterization of the beam geometry, the nodal positions in parametric $\xi$-, $\eta$ - and $\zeta$ - coordinates are obtained after dividing the parametric domain $W_{0} \times H_{0} \times L_{0}$ into equal $6 \times 10 \times 80$ hexahedral parts. Subsequently, the nodal positions 


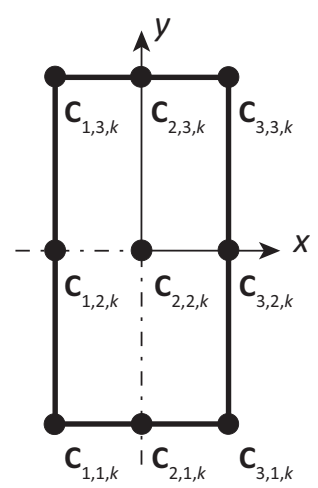

Figure 3.5: Control points for the NURBS surface at the $k$-th (with $k=1,2, \cdots, l$ ) location along the longitudinal direction ( $z$-direction) of the beam.

Table 3.2: Coordinates (in $\mathrm{m}$ ) and corresponding weights of the control points of the $k$-th (with $k=1,2, \cdots, l$ ) NURBS surface.

\begin{tabular}{llll}
\hline & $i=1$ & $i=2$ & $i=3$ \\
\hline$j=3$ & $\mathbf{C}_{1,3, k}=\left(-0.5,1, Z_{k}\right)$ & $\mathbf{C}_{2,3, k}=\left(0,1, Z_{k}\right)$ & $\mathbf{C}_{3,3, k}=\left(0.5,1, Z_{k}\right)$ \\
& $\omega_{1,3, k}=1$ & $\omega_{2,3, k}=1$ & $\omega_{3,3, k}=1$ \\
\hline$j=2$ & $\mathbf{C}_{1,2, k}=\left(-0.5,0, Z_{k}\right)$ & $\mathbf{C}_{2,2, k}=\left(0,0, Z_{k}\right)$ & $\mathbf{C}_{3,2, k}=\left(0.5,0, Z_{k}\right)$ \\
& $\omega_{1,2, k}=1$ & $\omega_{2,2, k}=1$ & $\omega_{3,2, k}=1$ \\
\hline$j=1$ & $\mathbf{C}_{1,1, k}=\left(-0.5,-1, Z_{k}\right)$ & $\mathbf{C}_{2,1, k}=\left(0,-1, Z_{k}\right)$ & $\mathbf{C}_{3,1, k}=\left(0.5,-1, Z_{k}\right)$ \\
& $\omega_{1,1, k}=1$ & $\omega_{2,1, k}=1$ & $\omega_{3,1, k}=1$ \\
\hline
\end{tabular}

of the FEM model are found by projecting the auxiliary mesh in the parametric domain to the physical domain using Eq. 3.14, see also Figure 3.2. These nodal positions are finally utilized to construct the $3 \mathrm{D}$ and $2.5 \mathrm{D}$ models of the cantilever beam, as illustrated in Figures 3.1(b) and (c), respectively. In accordance with the shape optimization procedure formulated in Eq. (3.60), the objective - the structural compliance $c$ - and constraints - the volume $V$ - are evaluated using the relations for the 3D FEM model introduced in Section 3.3.1. The relative element densities $\boldsymbol{\rho}$ are uniformly kept fixed at a value of 1 . In order to limit the computational demand of the 3D FEM model in the comparison study on shape optimization of $3 \mathrm{D}$ and $2.5 \mathrm{D}$ beam models, only the height $H$ of the beam is allowed to change along the beam length, whereby the cross-section is prescribed to remain rectangular. Note that this restriction in the shape design variables does not affect the nature and objectivity of the comparison study; in fact, it could have been introduced equally well as a geometrical constraint following from structural design considerations. It is further emphasized that it is straightforward to incorporate the width of beam cross-sections into the set of shape design variables, as is done for the coupled shape and topology optimization analysis of the $2.5 \mathrm{D}$ beam model considered in Section 3.6.2. In correspondence with the above arguments, the shape design variables a are formulated as 


$$
\mathbf{a}=\left[Y_{1,3,2}, Y_{1,3,3}, \cdots, Y_{1,3, k}\right]^{\mathrm{T}} \quad \text { with } \quad k=2,3, \cdots, l,
$$

where $Y_{1,3, k}$ is the $y$-coordinate of control point $\mathbf{C}_{1,3, k}$ illustrated in Figure 3.5 . The upper and lower bounds of this $y$-coordinate are set as $0.5 \mathrm{~m} \leq Y_{1,3, k} \leq 1.5 \mathrm{~m}$, which also apply to the $y$-coordinate of neighboring control points via the equality $Y_{1,3, k}=$ $Y_{2,3, k}=Y_{3,3, k}$. Further, the coordinates of the control points of the first NURBS surface $(k=1)$ are taken the same as those of the adjacent, second NURBS surface $(k=2)$; in fact, the first cross-section is only used for post-processing of the analysis results, and does not contribute to the optimization procedure, see also [117]. The values of the shape design variables a are efficiently updated by incorporating the analytical shape sensitivities presented in Section 3.5.2.1 in a Sequential Quadratic Programming (SQP) procedure that was implemented in the MATLAB solver fmincon. The shape optimization is assumed to be converged when the structural compliance meets the stop criterion $\left|c^{h+1}-c^{h}\right| \leq 1 \mathrm{e}-5$, with $h$ the iteration number, or when the shape design variables satisfy the criterion $\max \left(\left|\mathbf{a}^{h+1}-\mathbf{a}^{h}\right|\right) \leq 1 \mathrm{e}-5$, whereby $\mid$. | refers to the absolute value of a scalar or the absolute value of each specific component of a vector.

Alternatively, the optimization procedure can be carried out by modeling the cantilever beam as a $2.5 \mathrm{D}$ model. The $2.5 \mathrm{D}$ model is composed of 80 two-node beam elements along the beam $(z$-)axis (shown as a dotted black line in Figure 3.1(c)). The $z$-coordinate of the beam nodes is given by Eq. (3.93). The stiffness of each beam finite element is obtained from a 2D FEM analysis of the beam cross-section (evaluated at one of the beam element nodes). The meshes of the 81 cross-sections have a similar mesh density as in the 3D FEM model. In Table 3.2, the $z$-coordinate $Z_{k}$ (with $k=1,2, \cdots, 81$ ) of the control points depicted in Figure 3.5 essentially is irrelevant as a result of the two-dimensional character of the NURBS surface, and therefore is arbitrarily set to zero. The shape design variables a relate to the height of the cross-sections, and are presented by Eq. (3.94). The shape optimization procedure is performed in accordance with Eq. (3.60), for which the analytical shape sensitivities are provided in Section 3.5.1.1.

The results of the shape optimization procedures with the $3 \mathrm{D}$ and $2.5 \mathrm{D}$ beam models are shown in Figures 3.6 and 3.7. It can be observed that both models provide almost the same optimized shape, whereby the beam height near the clamped end, at which the bending moment is relatively large, is maximal and equal to $1.5 \mathrm{~m}$. Along the middle part of the beam the height gradually decreases, whereby it reaches the minimum value of $0.5 \mathrm{~m}$ close to the end at which the external load is applied. The convergence behavior of the shape optimization procedure for the two beam-type structures is illustrated in Figure 3.8 The convergence rate of the structural compliance $c$ for the 2.5D model is somewhat lower than for the 3D model, although the two models eventually provide the same minimum value of $c$. The computational time of the $2.5 \mathrm{D}$ model is about a factor of 70 lower than that of the $3 \mathrm{D}$ model, which illustrates that the $2.5 \mathrm{D}$ model is computationally much more efficient in this case. 


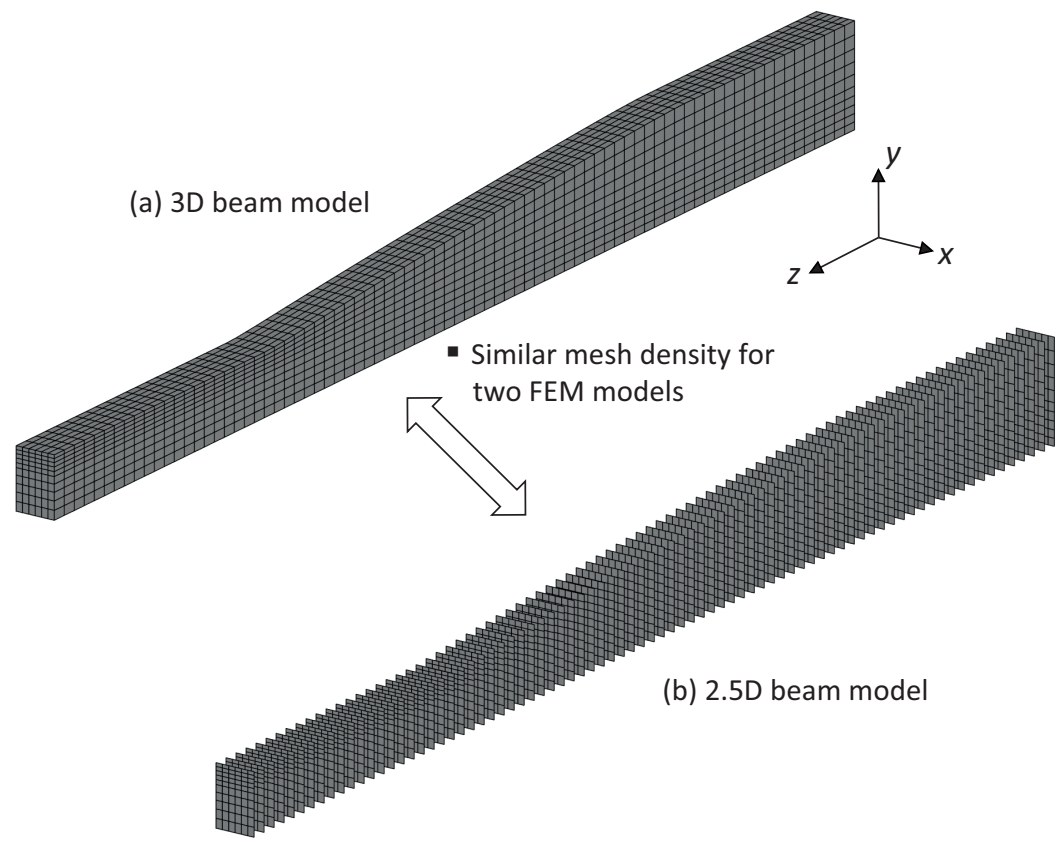

Figure 3.6: Local optimal designs by pure shape optimization using (a) 3D and (b) $2.5 \mathrm{D}$ beam modeling.

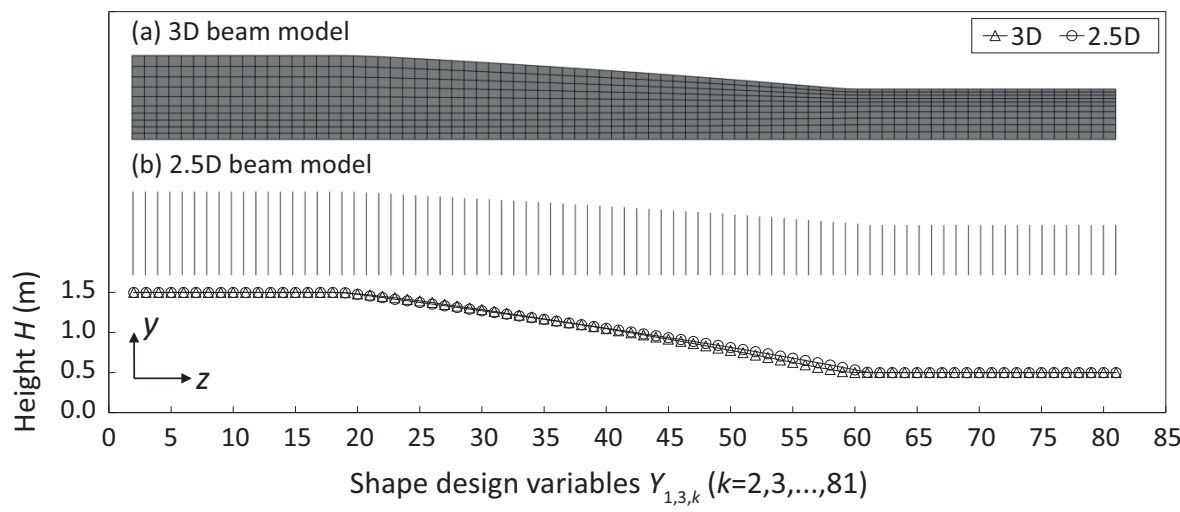

Figure 3.7: Comparison of the optimal height of the beam along the longitudinal direction, calculated by pure shape optimization for $3 \mathrm{D}$ (a) and 2.5D (b) beam models. 


\subsubsection{Topology optimization}

As a next step, the cantilever beam problem illustrated in Figure 3.4 is subjected to pure topology optimization, whereby the results computed with the $2.5 \mathrm{D}$ and $3 \mathrm{D}$ beam models are mutually compared. The geometry and FEM models of the problem are in accordance with the description in Section 3.6.1.1. In the topology optimization procedure the volume fraction $f_{r}$ appearing in Eq. (3.59) is set equal to 0.4. Further, the Interior-Point (IP) algorithm implemented in the MATLAB solver fmincon 69] is employed to update the element densities $\boldsymbol{\rho}$. The sensitivities required for the gradientbased topology optimization procedure are presented in Sections 3.5.1.2 and 3.5.2.2 for the $2.5 \mathrm{D}$ and $3 \mathrm{D}$ beam models, respectively. The topology optimization procedure is assumed to be converged when the structural compliance meets the stop criterion $\left|c^{g+1}-c^{g}\right| \leq 1 \mathrm{e}-5$, with $g$ the iteration number, or when the topology design variables satisfy the criterion $\max \left(\left|\boldsymbol{\rho}^{g+1}-\boldsymbol{\rho}^{g}\right|\right) \leq 1 \mathrm{e}-5$. In order to warrant an objective comparison of the results calculated for the $2.5 \mathrm{D}$ and $3 \mathrm{D}$ beam models, the filtering of possible checkerboard patterns, which is defined by Eq. (3.78) and may work out differently for the $2.5 \mathrm{D}$ and $3 \mathrm{D}$ beam models, is omitted here.

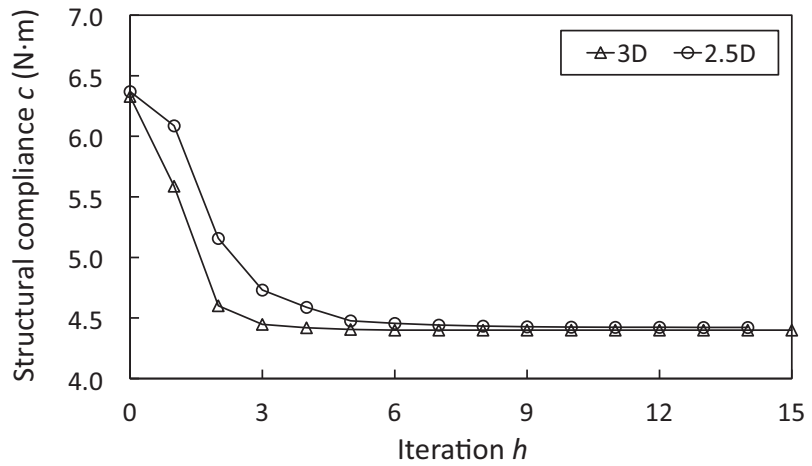

Figure 3.8: Convergence behavior of the compliance $c$ for pure shape optimization of 3D (triangles) and 2.5D (circles) beam models. The iteration number $h$ refers to the loop for shape optimization, see Figure 3.3

Figure 3.9 shows the convergence behavior of the structural compliance $c$ of the topology optimization process for the 2.5D (circles) and 3D (triangles) beam models. The overall convergence behavior and the corresponding compliance values clearly are similar for the two beam models. As a result of three-dimensional stress effects, some minor differences emerge in the relative density distributions at the clamped end and at the point of load application, see Figures 3.10 and 3.11 . The density distributions across the cross-sections illustrate that most of the material is located at the top and bottom of the beam height, which is logical for a beam structure loaded in bending. The optimized configurations presented in Figures 3.10 and 3.11 further show the presence of intermediate element densities with values around $\rho_{e}=0.5$ (green colour). Note that in topology optimization analyses the appearance of intermediate densities 
commonly is considered as undesirable, as it fades the distinction between a solid material (material density is unity) and a void (material density is zero). An efficient way to reduce intermediate densities in topology optimization results is by increasing the penalization factor $p$ in Eq. (3.26), see [98, which in the present work has been selected in accordance with the common value $p=3$ [43]. Alternative, more elaborate strategies that mitigate the appearence of intermediate densities have been presented in $94,121,122$. The main purpose of Figures 3.10 and 3.11 , however, is to objectively compare the optimized configurations computed for $3 \mathrm{D}$ and $2.5 \mathrm{D}$ beam models, for which the appearance of intermediate densities is insignificant. In addition, in the analyses performed with the coupled shape and topology optimization approach as presented in Section 3.6.2 the appearance of intermediate densities turns out to be negligible.

The computational time required for solving the 3D beam model is 1.4 times larger than the time needed for solving the $2.5 \mathrm{D}$ beam model, indicating that the reduction in spatial dimension here only provides a relatively small gain in computational efficiency as compared to the reduction of a factor of 70 found in Section 3.6.1.1 for pure shape optimization. This can be explained by considering the computational time of the topology optimization procedure in more detail. In particular, the time required for the structural analysis of the beam for the $2.5 \mathrm{D}$ model is one order of magnitude smaller than for the 3D model. However, this gain in time is counterbalanced by the fact that the time required for the sensitivity analysis of the $2.5 \mathrm{D}$ model is one order of magnitude larger than that of the 3D model. The latter result is caused by two reasons. Firstly, in 3D topology optimization the sensitivity of the global stiffness matrix can be efficiently obtained from the element stiffness matrices stored during the structural analysis, see Eqs. (3.88) and (3.89). In contrast, in 2.5D topology optimization, the calculation of the sensitivity of the global stiffness matrix requires the performance of a 1D FEM beam analysis, whereby the sensitivity of the stiffness matrix of each cross-section needs to be determined a priori, see Eqs. 3.61- 3.66 and (3.77), which obviously is computationally demanding. Secondly, the 1D beam FEM analysis must be repeated for the calculation of the sensitivity with respect to each element density. These two aspects thus make the topology sensitivity analysis for the $2.5 \mathrm{D}$ model computationally considerable more expensive than for the $3 \mathrm{D}$ model, and therefore result in only a relatively small decrease of the overall computational time by a factor of 1.4 .

It should be noted that in the $2.5 \mathrm{D}$ beam model the number of cross-sections has been chosen in accordance with the element density of the 3D beam model in longitudinal direction, in order to perform a consistent comparison of the simulation results at the cross-sectional level, see Figure 3.10. From the viewpoint of computational efficiency, however, the number of cross-sections in the 2.5D beam model could have been taken somewhat lower without significantly compromising on the accuracy of the simulation results. It can be further noticed that the initial compliances plotted in Figure 3.9 are different from those plotted in Figure 3.8, although the same initial beam configuration is used in the shape and topology optimization analyses. This is, because in the shape optimization analysis the relative element densities are kept fixed at a value of 1 , while the relative element densities in the topology optimization 
analysis are initially uniformly set equal to 0.4 , in correspondence with the constraint on the volume fraction, $f_{r}=0.4$.

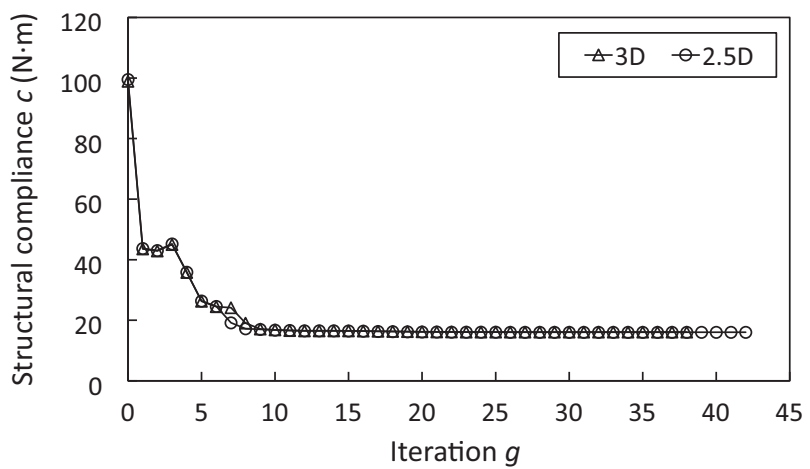

Figure 3.9: Convergence behavior of the compliance $c$ for pure topology optimization of $3 \mathrm{D}$ (triangles) and 2.5D (circles) beam models. The iteration number $g$ refers to the loop for topology optimization, see Figure 3.3

Figures 3.8 and 3.9 show that the structural compliances computed for the 2.5D and $3 \mathrm{D}$ beam models are very good agreement, both for shape optimization and topology optimization. In order to check the accuracy of the $2.5 \mathrm{D}$ model in more detail, for the case of topology optimization a cross-validation is performed, whereby the optimized density distribution computed by $2.5 \mathrm{D}$ model is transferred to the $3 \mathrm{D}$ FEM configuration, followed by calculating the compliance of the 3D FEM configuration. The compliance value following from this cross-validation procedure turns out to be $c=16.103 \mathrm{Nm}$, which is almost the same as the value of $c=16.096 \mathrm{Nm}$ calculated for the 3D beam model as shown in Figure 3.9. This confirms that it is trustworthy to perform the topology optimization procedure with the $2.5 \mathrm{D}$ beam model. Furthermore, for relatively large beam structures it may be worthwhile to maximize the computational benefit of the $2.5 \mathrm{D}$ beam model by selecting a minimal number of crosssections that corresponds to an adequate trade-off between computational accuracy and efficiency.

\subsubsection{Coupled shape and topology optimization case studies}

Now that the shape and topology implementations have been individually tested and validated, the cantilever beam problem depicted in Figure 3.4 is subjected to a coupled shape and topology procedure in accordance with the sequentially-coupled computational scheme depicted in Figure 3.3. The variation in shape and topology in the longitudinal beam direction is described by using 8 cross-sections, which are distributed along the beam length as shown in Figure 3.14(a). As explained before, for post-processing of the analysis results, the first cross-section at the clamped end of the cantilever beam (with index $k=1$ ) is taken the same as the adjacent second crosssection (with index $k=2$ ), and thus does not contribute to the optimization procedure 


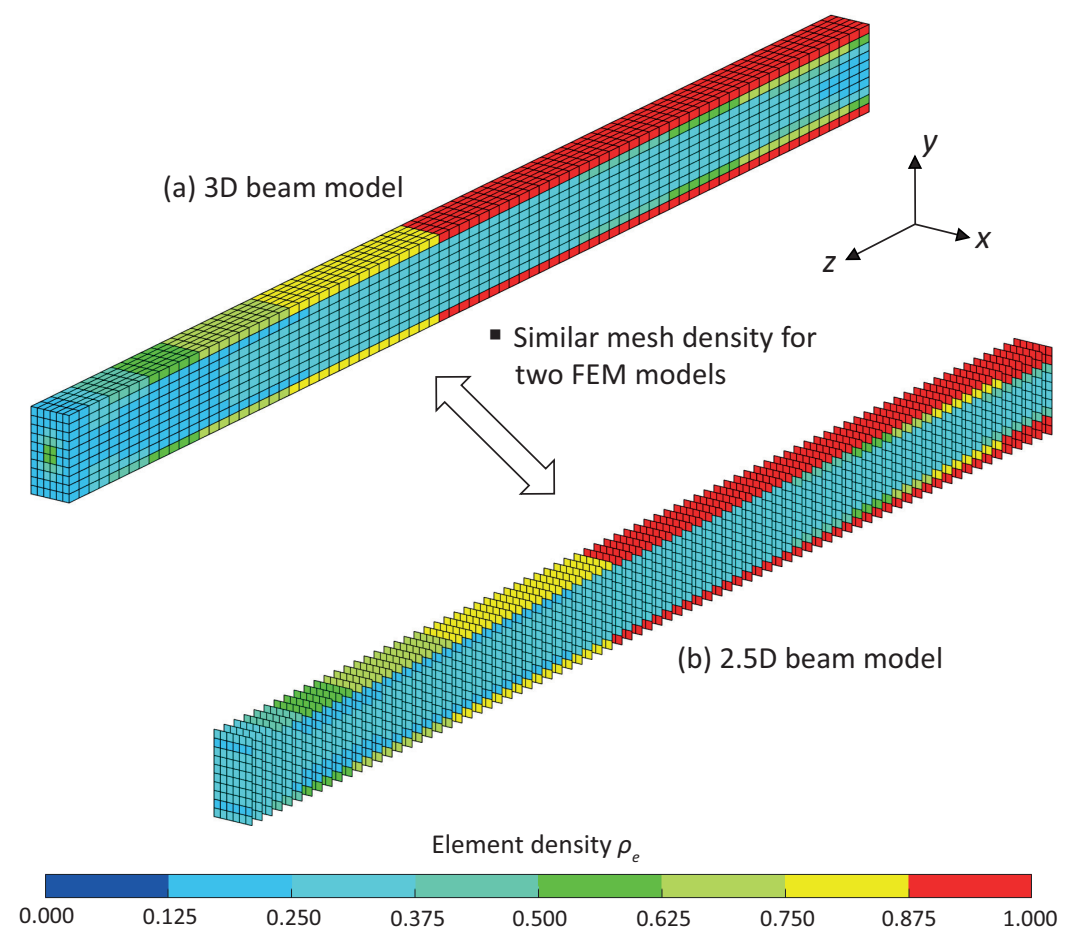

Figure 3.10: Comparison of the optimized designs obtained by pure topology optimization of 3D (a) and 2.5D (b) beam models. The filtering of possible checkerboard patterns via Eq. (3.78) is omitted here in order to warrant an objective comparison of the $3 \mathrm{D}$ and $2.5 \mathrm{D}$ modeling results.

[117. The locations of the control points of a cross-section and the corresponding coordinates are given in Figure 3.5 and Table 3.2. By leaving out the cross-section related to the clamped end, the shape design variables a of the 7 remaining cross-sections are assembled as

$$
\mathbf{a}=\left[\left(\mathbf{a}_{2}^{\mathrm{CS}}\right)^{\mathrm{T}},\left(\mathbf{a}_{3}^{\mathrm{CS}}\right)^{\mathrm{T}}, \cdots,\left(\mathbf{a}_{k}^{\mathrm{CS}}\right)^{\mathrm{T}}\right]^{\mathrm{T}} \quad \text { with } \quad k=2,3, \cdots, 8,
$$

in which

$$
\mathbf{a}_{k}^{\mathrm{CS}}=\left[X_{1,1, k}, Y_{1,1, k}, Y_{2,1, k}, X_{1,2, k}\right]^{\mathrm{T}},
$$

where $X_{1,1, k}$ and $Y_{1,1, k}$ are the $x$ - and $y$-coordinates of control points $\mathbf{C}_{1,1, k}$, and $X_{1,2, k}$ and $Y_{2,1, k}$ are the $x$ - and $y$-coordinates of control points $\mathbf{C}_{1,2, k}$ and $\mathbf{C}_{2,1, k}$, respectively. Note that the control points at the corners of a cross-section can move in both the $x$ - and $y$-directions, while the control points at the half-width and half-height of the 

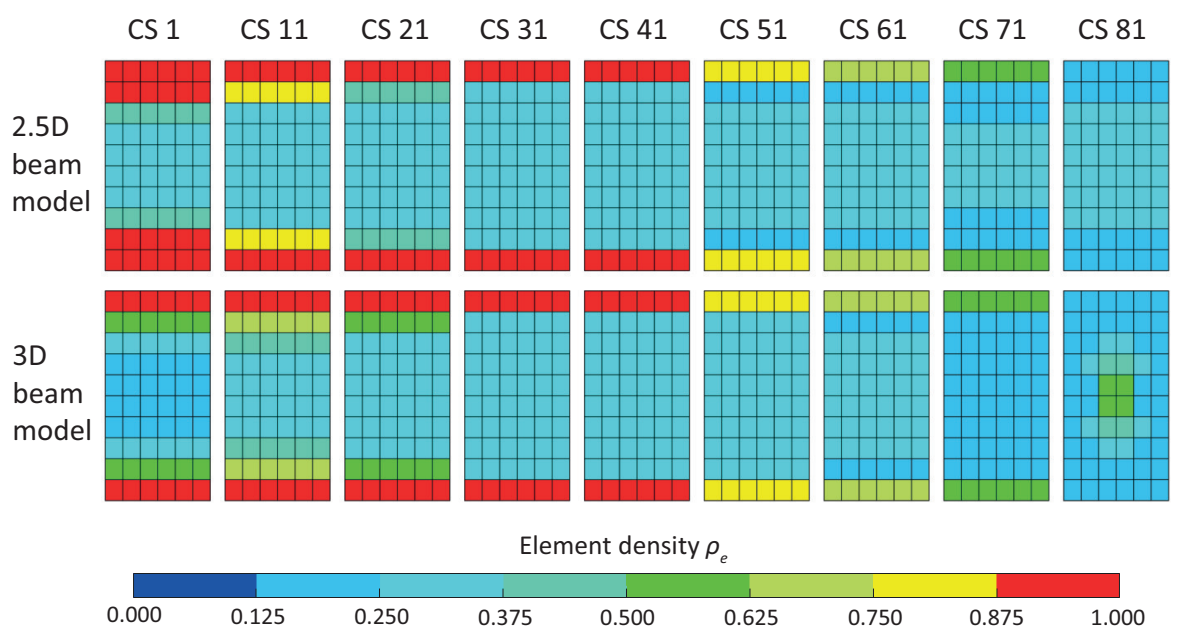

Figure 3.11: Cross-sectional (CS) relative densities obtained by pure topology optimization of 2.5D (top) and 3D (bottom) beam models. The number of each cross-section denotes its location along the longitudinal direction of the beam, in agreement with the $z$-axis in Figure 3.7. The filtering of possible checkerboard patterns via Eq. 3.78 is omitted here in order to warrant an objective comparison of the $2.5 \mathrm{D}$ and $3 \mathrm{D}$ modeling results.

cross-section boundaries can only move in the $y$-direction and $x$-direction, respectively. The control points of a cross-section are assumed to be line-symmetric with respect to the $x$ - and $y$-axes, so that the locations of control points $\mathbf{C}_{3,1, k}, \mathbf{C}_{3,2, k}, \mathbf{C}_{1,3, k}, \mathbf{C}_{2,3, k}$ and $\mathbf{C}_{3,3, k}$ are prescribed by the corresponding control points above. The lower and upper bounds of $\mathbf{a}_{k}^{C S}$ are, respectively, defined as $l b=[-1.5,-1.5,-2,-2]$ and $u b=$ $[-0.2,-0.2,-0.2,-0.2]$. The parametric domain of the NURBS surface that defines a cross-section is divided into $12 \times 20$ equally sized, rectangular parts, which define the grid for the 2D auxiliary FEM mesh. In the longitudinal direction the model uses a discretization by 120 2-node beam elements, for which the $z$-coordinates are given by Eq. 3.93 with $l=121$. The stiffness $\mathbf{k}_{e}^{\mathrm{b}}$ of each beam element $e$ is computed from the stiffness of the corresponding cross-section $\mathbf{K}_{e}^{\mathrm{s}}$ via Eq. 3.34). Accordingly, under a stepwise change in cross-sectional properties the optimized solution corresponds to a shape that varies linearly in the longitudinal direction. The volume fraction in Eq. (3.58) is defined as $f_{r}=0.4$. The structural compliance $c$ and the beam volume $V$ are calculated from the beam FEM model. The SQP and IP algorithms in the MATLAB solver fmincon are used to iteratively update the shape design variables a and element relative densities $\boldsymbol{\rho}$ appearing in Eq. (3.58). The convergence criteria adopted for the individual shape and topology optimization steps are the same as those defined in Section 3.6.1. In addition, the outer loop of the coupled optimization procedure, which has been illustrated in the flowchart in Figure 3.3 , is assumed to be converged when the structural compliance meets the condition $\left|c^{w+1}-c^{w}\right| \leq 1 \mathrm{e}-5$, where $w$ is the iteration number of the outer loop. Finally, the shape and topology sensitivities of the objective are computed with the expressions given in Section 3.5.1, whereby the 


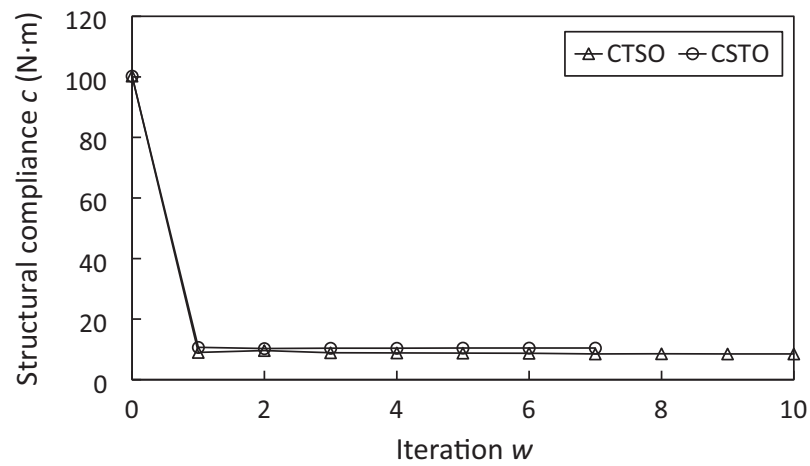

Figure 3.12: Convergence behavior for compliance $c$ for coupled shape and topology optimization with CTSO (triangles) and CSTO (circles) update schemes. The iteration number $w$ refers to the outer loop of the staggered update scheme, see Figure 3.3

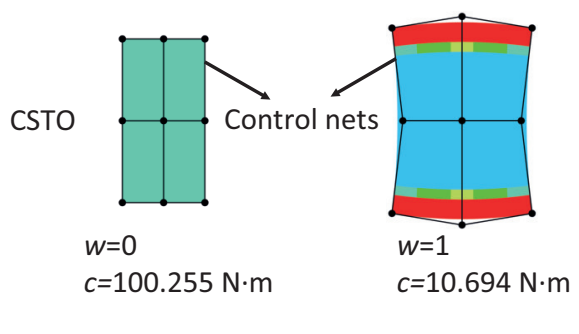

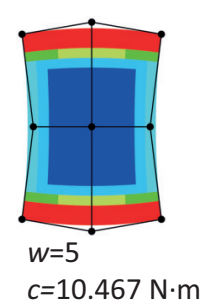

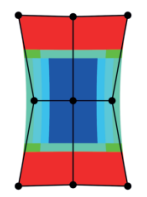

$$
w=5
$$

$c=8.754 \mathrm{~N} \cdot \mathrm{m}$

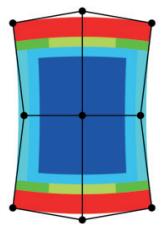

$w=7$ $c=10.467 \mathrm{~N} \cdot \mathrm{m}$

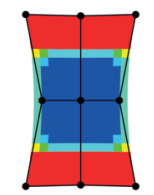

$w=10$

$c=8.516 \mathrm{~N} \cdot \mathrm{m}$ $w=1$

$c=8.993 \mathrm{~N} \cdot \mathrm{m}$

Element density $\rho_{e}$

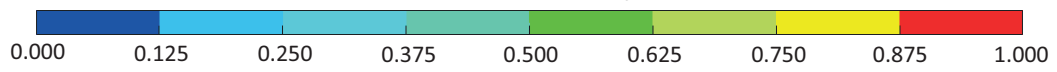

Figure 3.13: Evolution of the structural compliance $c$ and the geometry of the first crosssection at the clamped beam end during the convergence process for the CSTO and CTSO update schemes. The NURBS control points are indicated by the black dots, and $w$ is the iteration of the outer loop of the update scheme, see Figure 3.3.

2D sensitivity filter formulated in Eq. 3.78 is applied in the topology optimization procedure. The filter size is set to $r_{\min }=1.5 \sqrt{V_{e}}$, where $V_{e}$ is the area of element $e$ [112. The convergence behavior for the structural compliance $c$ is shown in Figure 3.12 The CTSO solution procedure that starts with a topology optimization step takes more iterations $(w=10)$ than the CSTO solution procedure that starts with a 
shape optimization step $(w=7)$. Figure 3.13 shows the evolution of the first crosssection at the clamped beam end during the convergence process for the CSTO and CTSO update schemes. The evolution of the cross-section clearly visualizes that the CTSO and CSTO update sequences during the convergence process follow a different search path in the solution space of design variables, eventually leading to somewhat different local minima (or optimized compliances), i.e., $c=10.467 \mathrm{Nm}$ (CSTO) and $c=8.516 \mathrm{Nm}$ (CTSO).

Figures 3.14 and 3.15 illustrate the optimized designs predicted by CSTO and CTSO sequences. For the FEM discretizations chosen here, the two solution procedures result in a comparable design concept, namely a tapered beam with a hollow cross-section composed of relatively thin vertical webs and thick horizontal flanges. The cross-section computed by the CSTO solution procedure has a smaller height-towidth ratio than the cross-section following from the CTSO procedure. Further, the beam structure following from the CSTO update procedure contains one cross-section (with index $k=4$ ) with an extra vertical web.

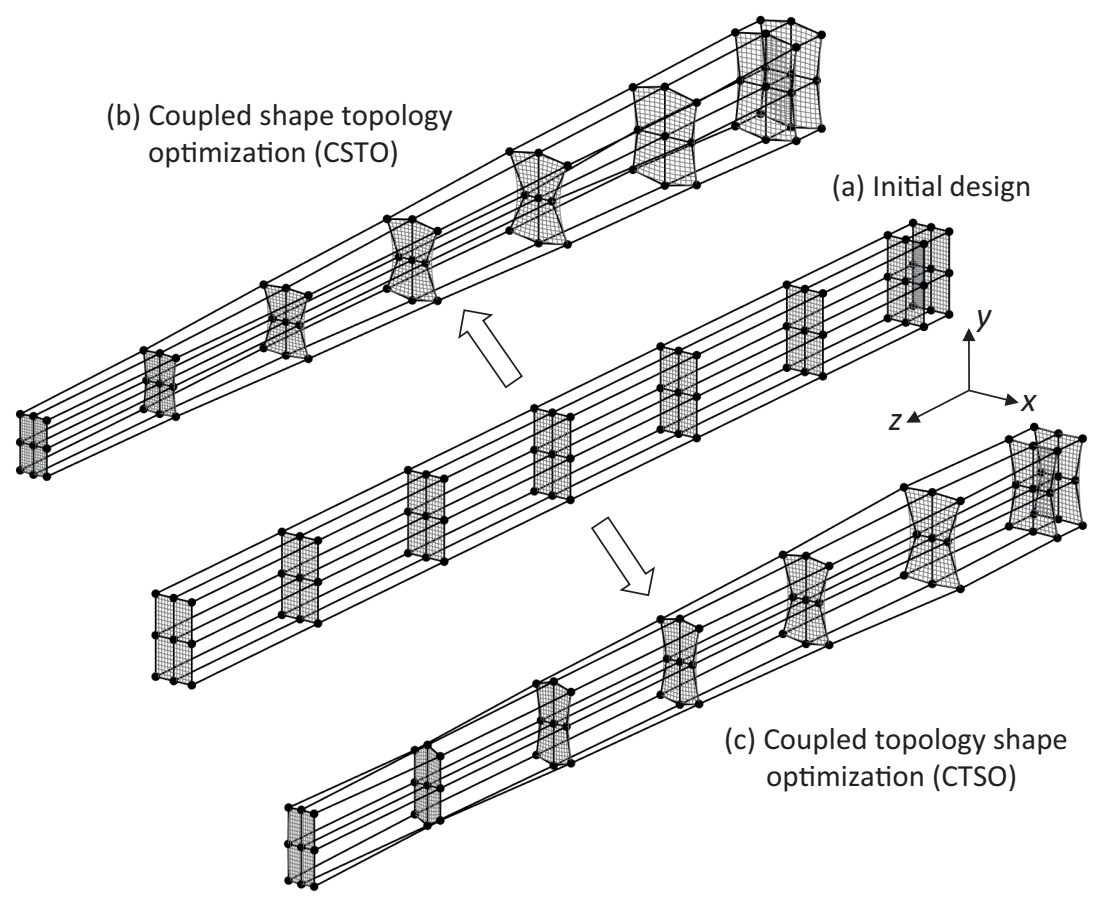

Figure 3.14: Optimized control nets of 2.5D beam model. Initial design (a) and solutions obtained by coupled shape and topology optimization using CSTO (b) and CTSO (c) update schemes.

In summary, in structural optimization problems, the landscape of solutions is determined by the objective function(s), the design variables and constraints, the characteristics of the boundary value problem and the FEM discretization, which 


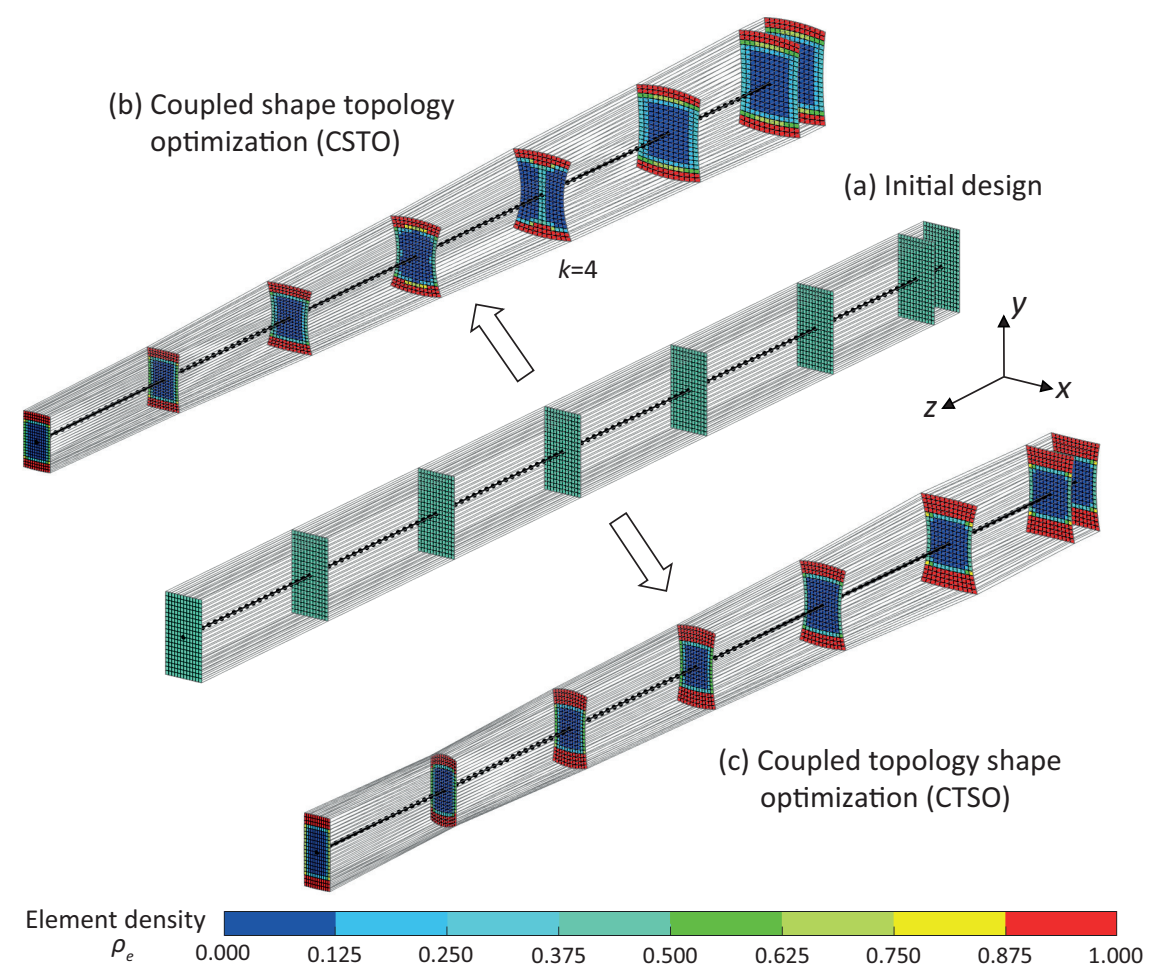

Figure 3.15: Optimized configurations of 2.5D beam model. Initial design (a) and solutions obtained by coupled shape and topology optimization using CSTO (b) and CTSO (c) update schemes.

typically leads to a large number of local minima, see e.g., 98. The above comparison of the analyses performed with the CTSO and CSTO update schemes illustrates that the convergence speed and the specific local minimum computed (i.e., the optimized compliance) are to some extent sensitive to the algorithmic features of the numerical update scheme applied. One possible reason for this may be the scale difference of the design variables for shape optimization and topology optimization. Therefore, for the analyses in [119, 120, the shape design variables have been normalized such that they range between zero and one, by which the scale difference in the shape and topology design variables vanishes.

\subsection{Conclusions}

A coupled gradient-based optimization framework is presented that simultaneously optimizes the outer shape and the internal topology of beam-type structures by using a staggered update procedure. The objective function refers to the structural 
compliance, which is evaluated using 2.5D and 3D FEM models. In the 2.5D model, standard 2-node beam elements are used along the longitudinal direction of the beam, whereby the cross-sectional properties of the beam elements are calculated from additional 2D FEM analyses. Conversely, in the 3D model the beam geometry is simulated using $3 \mathrm{D}$ continuum elements. The shape of the beam-type structures is parameterized using NURBS, with the shape design variables being represented by the NURBS control points. The topology design variables are reflected by the relative densities assigned to each finite element. The design variables are iteratively updated by applying NURBS-based shape and density-based topology optimization techniques, which use analytic shape and topology sensitivities that ensure an accurate and computationally efficient solution procedure. A comparison study of a cantilever beam problem subjected to pure shape optimization and pure topology optimization illustrates that the $2.5 \mathrm{D}$ and $3 \mathrm{D}$ beam models lead to similar shape and topology designs, but that the $2.5 \mathrm{D}$ beam model has a significantly higher computational efficiency. Specifically, the computational times for the 2.5D model are about a factor 70 (shape optimization) and 1.4 (topology optimization) lower than for the 3D model, which indicates that in the coupled optimization approach the optimization of the shape provides the largest contribution to the higher computational efficiency of the $2.5 \mathrm{D}$ model. The coupled shape and topology optimization analysis subsequently performed on the $2.5 \mathrm{D}$ cantilever beam model demonstrates that the specific order at which the alternating shape and topology optimization increments are performed in the staggered update procedure turns out to have some influence on the final computational result for the boundary value problem considered. Further, the convergence speed for the optimization procedure starting with an incremental topology optimization step appears to be somewhat lower than that resulting from starting with an incremental shape optimization step, although it may be reasonably expected that this feature is problem dependent. Despite these differences, the final beam structures following from the two staggered update schemes illustrate how the shape and topology can be adequately optimized in an integrated, coupled fashion. 


\section{Chapter 4}

\section{Aerostructural design of turbine blades}

This chapter is based on the journal paper:

Wang, Z., Suiker, A. S. J., Hofmeyer, H., van Hooff, T., Blocken, B. (2020). Coupled aerostructural shape and topology optimization of horizontal-axis wind turbine rotor blades. Energy Conversion and Management, 212, 112621.

Traditionally, Aerodynamic Shape Optimization (ASO) and Structural Topology Optimization (STO) are considered as two separate, consecutive stages in the aerostructural design process of wind turbine rotor blades. Since such a modeling strategy does not adequately account for the coupling effects between the blade aerodynamics and its structural performance, in this chapter a Coupled Multi-objective Shape and Topology Optimization (CMSTO) approach is presented, which simultaneously optimizes the outer shape and the interior structural layout of the turbine blade from aerodynamic and structural requirements. The framework uses the weighted sum method, whereby the sum of the aerodynamic and structural objectives multiplied by specific weighting factors is minimized employing an incremental-iterative update procedure. The coupled optimization process is performed by sequentially carrying out shape and topology optimization steps for beam-type structures, in accordance with a staggered scheme. For the aerodynamic and structural optimizations the rotor power coefficient and the blade structural compliance are considered as the objectives, respectively. The aerodynamic response of the blade is evaluated by the Blade Element Momentum (BEM) method, which, together with a reduced beam-type Finite Element Method (FEM) model that simulates the structural response, facilitates the application of a gradient-based algorithm with analytical sensitivities. Accordingly, the computa- 
tional efficiency of the CMSTO framework is warranted. The shape design variables are characterized by the locations of Non-Uniform Rational B-Splines (NURBS) control points that parameterize the blade outer shape. The topology design variables are represented by the relative densities assigned to the finite elements modeling the blade cross-sections, in correspondence with the Solid Isotropic Material with Penalization (SIMP) method. The CMSTO framework is used to optimize the NREL 5MW reference rotor blade, whereby the results are compared to those obtained from a separate ASO-STO approach and a pure STO approach. The rotor power coefficients calculated by the CMSTO and ASO-STO approaches are about $4 \%$ larger than that of the reference rotor blade. In addition, the blade structural compliance computed by the CMSTO approach is reduced by an extra $16 \%$ and $41 \%$ compared to the compliances found by ASO-STO and pure STO approaches, respectively. Such significant improvements clearly demonstrate the benefits of the CMSTO approach in the quest of wind turbines with higher power output and better structural performance.

\subsection{Introduction}

Over the past decades the optimization of the aerostructural performance of HorizontalAxis Wind Turbines (HAWTs) has been an important subject of investigation [42, whereby the main challenge has been to design turbine blades characterized by i) a high power output, ii) a high stiffness, and iii) a low mass. These three objectives typically are conflicting: Maximizing the power output of turbine blades commonly results in an increase of the blade mass [36, 37] and an increase of the aerodynamic loads 38. Consequently, the structural deformation of the blade increases [39, which in turn puts more stringent requirements on its effective stiffness. Additionally, the use of a low amount of material opposes a high structural stiffness when the internal material distribution (i.e., the structural layout) of the blade is kept fixed [40, 41].

The optimization of the aerostructural performance of wind turbines is usually carried out in two consecutive stages [15], which correspond to the aerodynamic design of the blade outer shape and the structural design of the interior layout of the blade. During the first stage, a shape optimization process is performed, in which the blade length, airfoil profile and chord and twist distributions of the blade are varied to achieve an optimal aerodynamic performance [49, 50, 55, 56. The aerodynamic loads acting on the rotor can be calculated via Computational Fluid Dynamics (CFD) simulations or via the Blade Element Momentum (BEM) method [123. Although CFD models provide an accurate representation of the complex aerodynamics of wind turbine blades [124-126], the high computational costs involved still withhold their incorporation in optimization frameworks [34. During the second stage, the interior layout of the blade is optimized using the aerodynamic loads from the first stage as input. Traditionally, the optimization process starts from a hollow airfoil, whereby subsequently reinforcements (spar caps and shear webs) are added to improve the bending, shear, and torsional resistances of the blade [57. The internal blade layout is usually optimized by adapting the thickness of the airfoil and the size and location of the reinforcements via size and shape optimization methods [45]47]. However, 
this strategy does not naturally allow for the introduction of new topological concepts for the blade layout, which is the reason that topology optimization techniques were introduced to find the optimal shapes and locations for the stiffeners of the turbine blades [53. In addition, the design of wind turbine blades has led to the development of advanced topology optimization methods [127-129], which account for the shape-dependent pressure load resulting from the wind loading.

Since the shape and topology optimization processes typically are performed in two consecutive stages, the coupling effects between the blade aerodynamics and its structural performance are not adequately taken into account. The aerostructural design and multi-objective optimization techniques presented in [35, 39, 58, 60, could overcome this limitation, but these frameworks so far have only been applied to specific blade layouts for which the outer shape is optimized together with the size and location of the stiffeners. In addition, the update of the design variables for most of the optimization techniques is performed with gradient-free optimization algorithms, such as genetic algorithms [36, 41, 59, 60. These algorithms facilitate an easy and flexible numerical implementation [37] and the possibility of finding a global optimum, but may also exhibit a poor convergence behavior [36] and thus are computationally expensive, especially if the number of design variables is relatively large 61. From these viewpoints, gradient-based optimization methods, for which the sensitivities are derived in analytical form, are preferable.

In accordance with the aforementioned state of the art and challenges for the aerostructural design of large wind turbine blades, this chapter develops a Coupled Multi-objective Shape and Topology Optimization (CMSTO) framework that is able to simultaneously optimize the outer shape and interior topological layout of wind turbine rotor blades from aerodynamic and structural requirements. The framework uses the weighted sum method 130, whereby the sum of the aerodynamic and structural objectives multiplied by specific weighting factors is minimized. The rotor blade is simulated as a 2.5D reduced beam-type structure [117]; the efficiency and accuracy of this beam model have been validated in [131] through an extensive comparison with 3D beam models. Non-Uniform Rational B-Splines (NURBS) 89 are employed to parametrically describe the blade outer shape, which not only guarantees the smoothness of the blade shape, but also enables an efficient control of the geometry using only a few control points. The aerodynamic response of the blade is evaluated by the BEM method, for which the analytic sensitivities of the aerodynamic parameters are reported in the literature [58, 61, 132] and the numerical code is available open source [132. The combination of the BEM method with a reduced beam-type FEM model supports the use of a gradient-based algorithm with analytical sensitivities, as a result of which the computational efficiency of the present optimization framework is warranted. With the coupled optimization method proposed in this paper, a better trade-off between the blade aerodynamic and structural performances can be achieved, which will be demonstrated through a practical case study related to a NREL 5MW reference rotor blade.

This chapter is organized as follows. Section 4.2 outlines the coupled aerostructural shape and topology optimization framework. The solution strategy and the numerical setup for the aerostructural optimization of a NREL 5MW reference rotor blade are 


\subsection{Coupled aerostructural optimization model}

described in Section 4.3, and the numerical results are presented in Section 4.4. Finally, Section 4.5 summarizes the main conclusions of the optimization study.

\subsection{Coupled aerostructural optimization model}

In this section a Coupled Multi-objective Shape and Topology Optimization (CMSTO) model is presented that can be used for the aerostructural design of wind turbine blades. The model uses the weighted sum method [130, whereby the sum of the aerodynamic and structural objectives multiplied by specific weighting factors is minimized using an incremental-iterative numerical update procedure. The coupled optimization process is performed by sequentially carrying out shape and topology optimization steps for a beam-type structure, in accordance with the staggered scheme presented in [112, 131. For the aerodynamic and structural optimizations the rotor power coefficient and the blade structural compliance are considered as the objectives, respectively. The shape design variables are characterized by the locations of NURBS control points that parameterize the blade outer shape. The topology design variables are represented by the relative densities assigned to the finite elements modeling the blade cross-sections, in correspondence with the Solid Isotropic Material with Penalization (SIMP) method 43. Accordingly, the coupled aerostructural optimization problem can be formulated as

$$
\begin{array}{cl}
\min _{\mathbf{a}, \boldsymbol{\rho}} & f(\mathbf{a}, \boldsymbol{\rho})=\omega \frac{C_{\mathrm{p} 0}}{C_{\mathrm{p}}(\mathbf{a})}+(1-\omega) \frac{c(\mathbf{a}, \boldsymbol{\rho})}{c_{0}}, \\
\text { subject to } & \frac{V(\mathbf{a}, \boldsymbol{\rho})}{V_{0}} \leq f_{\mathrm{r}}, \\
& \mathbf{A} \mathbf{a} \leq \mathbf{b}, \\
& \frac{A_{n}(\mathbf{a}, \boldsymbol{\rho})}{A_{n}^{0}(\mathbf{a})} \leq f_{\mathrm{r}}, \quad n=1, \ldots, N, N=1, \ldots, S, S \\
\text { with } & 0 \leq \frac{a_{s}-l_{s}}{u_{s}-l_{s}} \leq 1, \quad e=1, \ldots, E, \\
& 0 \leq \rho_{\min } \leq \rho_{e} \leq 1, \quad e=1,
\end{array}
$$

where the rotor power coefficient $C_{\mathrm{p}}$ and the blade structural compliance $c$ are the two objectives of the objective function $f$. The objectives are weighted by a weighting factor $\omega$, with $0 \leq \omega \leq 1$. Since the objectives have different units, they are normalized by their initial values $C_{\mathrm{p} 0}$ and $c_{0}$. Further, $\mathbf{a}$ is the vector containing the normalized blade shape design variables $a_{s}$, with their lower and upper bounds given by $l_{s}$ and $u_{s}$, respectively, and $S$ representing the total number of shape design variables. The vector $\boldsymbol{\rho}$ contains the topology design variables, $\rho_{e}$ is the relative density assigned to finite element $e, E$ is the total number of finite elements, and $\rho_{\min }$ is the minimum element density (which needs to be non-zero in order to avoid a singular stiffness matrix). Note that the shape design variable $a_{s}$ has been normalized in order to range between zero and one, by which the scale difference in the ranges for the shape and topology design 
variables vanishes. The parameters $V$ and $V_{0}$ represent the total material volume and initial blade volume, respectively, with their maximum fraction a priori prescribed as $f_{\mathrm{r}}$, which is used for determining the initial value of the density $\rho_{e}$ of each element. The matrix $\mathbf{A}$ and vector $\mathbf{b}$ include the linear inequality constraints for the shape design variables a. Moreover, $A_{n}^{0}$ refers to the area of a specific blade cross-section $n$, and $A_{n}$ represents the total area of material distributed across this cross-section. The maximum ratio of $A_{n}$ and $A_{n}^{0}$ is controlled by the prescribed fraction $f_{\mathrm{r}}$, and $N$ is the total number of blade cross-sections.

In the coupled optimization formulation summarized in Eq. 4.1), the structural compliance $c$ is minimized together with the inverse of the power coefficient $C_{\mathrm{p}}$ for a given initial blade outer shape and wind conditions. The structural compliance $c$ is defined by

$$
c=\mathbf{f}^{\mathrm{T}} \mathbf{u}
$$

with $\mathbf{f}$ and $\mathbf{u}$ the global force and displacement vectors of the FEM model for the rotor blade, which are related as

$$
\mathbf{f}=\mathbf{K u}
$$

where $\mathbf{K}$ is the overall stiffness matrix. Note that minimizing the inverse of the power coefficient is equivalent to maximizing the power coefficient. Similarly, minimizing the structural compliance $c$ is equivalent to maximizing the overall stiffness of the rotor blade. Accordingly, by solving the coupled multi-objective optimization problem formulated by Eq. 4.1, a fixed amount of material can be optimally placed in a geometrically optimized design domain to achieve both a high rotor power coefficient and a high blade stiffness.

\subsection{Numerical solution strategy}

The coupled optimization problem formulated by Eq. 4.1 is solved numerically using a staggered scheme. As illustrated in Figure 4.1 the blade outer shape and the structural layout are updated in an incremental-iterative fashion by alternatively performing shape and topology optimization steps. The formulation and numerical performance of this staggered scheme are presented in [112, 131].

In the numerical update scheme, first a multi-objective optimization step on the outer shape of the turbine blade is performed, formulated as 


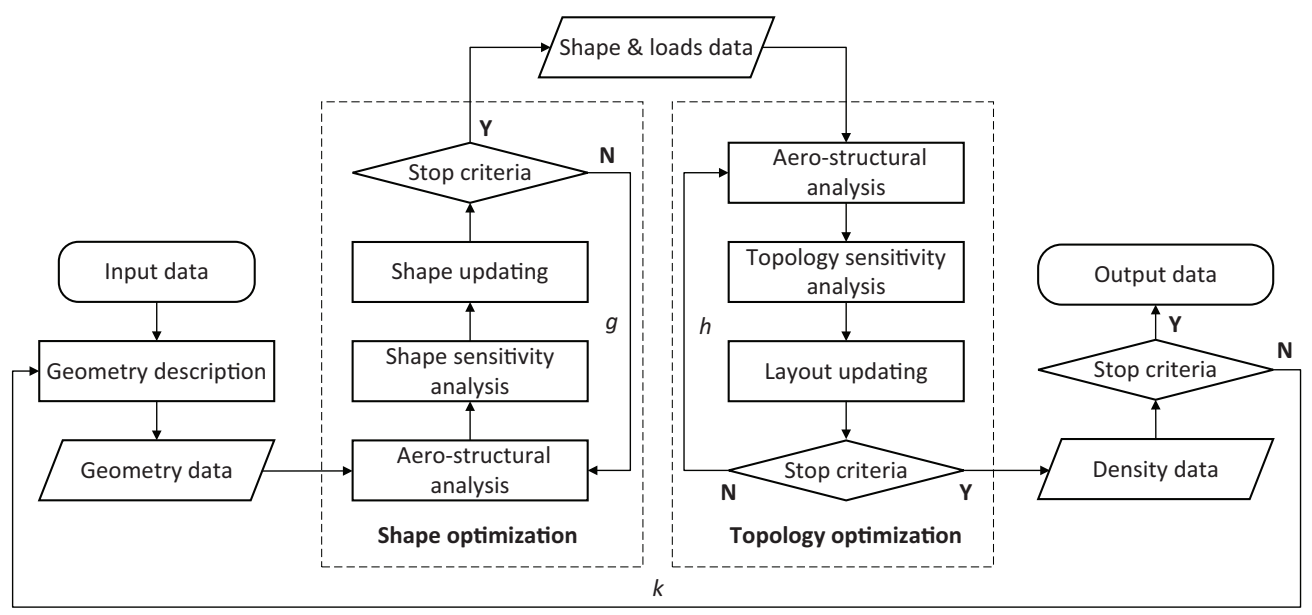

Figure 4.1: Solution strategy for coupled shape and topology optimization. Iterations $g$ and $h$ refer to the shape and topology optimization sub-loops, respectively, and iteration $k$ refers to the outer loop.

$$
\begin{array}{cl}
\min _{\mathbf{a}} & f(\mathbf{a})=\omega \frac{C_{\mathrm{p} 0}}{C_{\mathrm{p}}(\mathbf{a})}+(1-\omega) \frac{c(\mathbf{a})}{c_{0}}, \\
\text { subject to } & \frac{V(\mathbf{a})}{V_{0}} \leq f_{\mathrm{r}}, \\
& \mathbf{A} \mathbf{a} \leq \mathbf{b}, \\
& \frac{A_{n}(\mathbf{a})}{A_{n}^{0}(\mathbf{a})} \leq f_{\mathrm{r}}, \quad n=1, \ldots, N, \\
\text { with } & 0 \leq \frac{a_{s}-l_{s}}{u_{s}-l_{s}} \leq 1, \quad s=1, \ldots, S,
\end{array}
$$

during which the element densities $\rho$ are kept fixed. After Eq. 4.4 is solved, a topology optimization step is performed whereby the updated design variables a of the blade outer shape are temporarily kept fixed:

$$
\begin{array}{cll}
\min _{\boldsymbol{\rho}} & f(\boldsymbol{\rho})=\omega \frac{C_{\mathrm{p} 0}}{C_{\mathrm{p}}}+(1-\omega) \frac{c(\boldsymbol{\rho})}{c_{0}}, \\
\text { subject to } & \frac{V(\boldsymbol{\rho})}{V_{0}} \leq f_{\mathrm{r}}, & n=1, \ldots, N, \\
& \frac{A_{n}(\boldsymbol{\rho})}{A_{n}^{0}} \leq f_{\mathrm{r}}, \\
& 0 \leq \rho_{\text {min }} \leq \rho_{e} \leq 1, \quad e=1, \ldots, E .
\end{array}
$$

Note that the maximum value of the weighting factor $\omega$ in Eq. 4.5 should be smaller than 1 in order to carry out the topology optimization step. The optimized blade outer 
shape, obtained from solving Eq. 4.4, might not be structurally optimal anymore after the interior blade topology, represented by the element densities $\boldsymbol{\rho}$, has been optimized by solving Eq. 4.5. Correspondingly, the shape and topology optimization steps need to be alternately repeated until the stop criteria of the outer loop $k$ are met, see Figure 4.1. The details of the individual steps in the solution strategy are provided in the subsequent section by means of an illustrative example.

\subsubsection{Geometry description}

The features of the coupled optimization framework are demonstrated by selecting the National Renewable Energy Laboratory (NREL) 5MW wind turbine rotor as a reference model, of which the design details are well documented in [133, 134. The blade geometry of the wind turbine rotor is described by 19 cross-sections along the blade length, see Figure 4.2 , with the geometry in between two adjacent cross-sections being determined by linear interpolation. The first two cross-sections near the blade root (i.e., cross-sections 0 and 1) are circular, in order to warrant a decent connection between the blade and the rotor. The blade reference geometry is constructed from the 6 airfoil types depicted in Figure 4.3 , whereby the geometry of each of these airfoil types is described by $x$ - and $y$-coordinates that are normalized by the chord length $l$, i.e., $x_{\mathrm{a}}=x / l$ and $y_{\mathrm{a}}=y / l$. The final shape of the airfoil at each of the cross-sections is determined by upscaling the normalized shape presented in Figure 4.3 using the actual chord length $l$, and subsequently rotating the airfoil in accordance with the corresponding twist angle $\beta$. The distributions of the chord length and twist angle along the blade length are plotted in Figures 4.4 and 4.5 , respectively, as taken from 133, 134. Each distribution was fitted by a NURBS curve equipped with 5 control points. The initial locations of the control points indicated in Figures 4.4 and 4.5 were calculated such that the actual chord and twist distributions of the reference blade were accurately matched. In this way, the shape design variables a of the blade are fully determined by the control point locations of the blade chord and twist distributions. During the optimization procedure the first control point (located close to the blade root) and last control point (located at the blade tip) of the chord and twist distributions are allowed to move only vertically, in correspondence with the assumption that the length of the blade remains constant. The other three control points can move both horizontally and vertically, but are not allowed to intersect or pass each other. Additionally, from general design considerations the blade chord distribution after the second control point is enforced to monotonically decrease towards the blade tip [37. In other words, the chord lengths related to the second, third, fourth and fifth control points are required to successively decrease in value. More details on the use of NURBS for describing structural geometries can be found in 131 and references therein.

The above-described mapping from a normalized airfoil geometry to the actual blade cross-section is illustrated in Figure 4.6, and can be described by means of a geometry function $\mathbf{G}$ given by

$$
\mathbf{G}\left(\mathbf{p}_{\mathrm{a}}\right)=l \mathbf{R}\left(\mathbf{p}_{\mathrm{a}}-\mathbf{o}_{\mathrm{c}}\right)+\mathbf{o}_{\mathrm{b}} .
$$




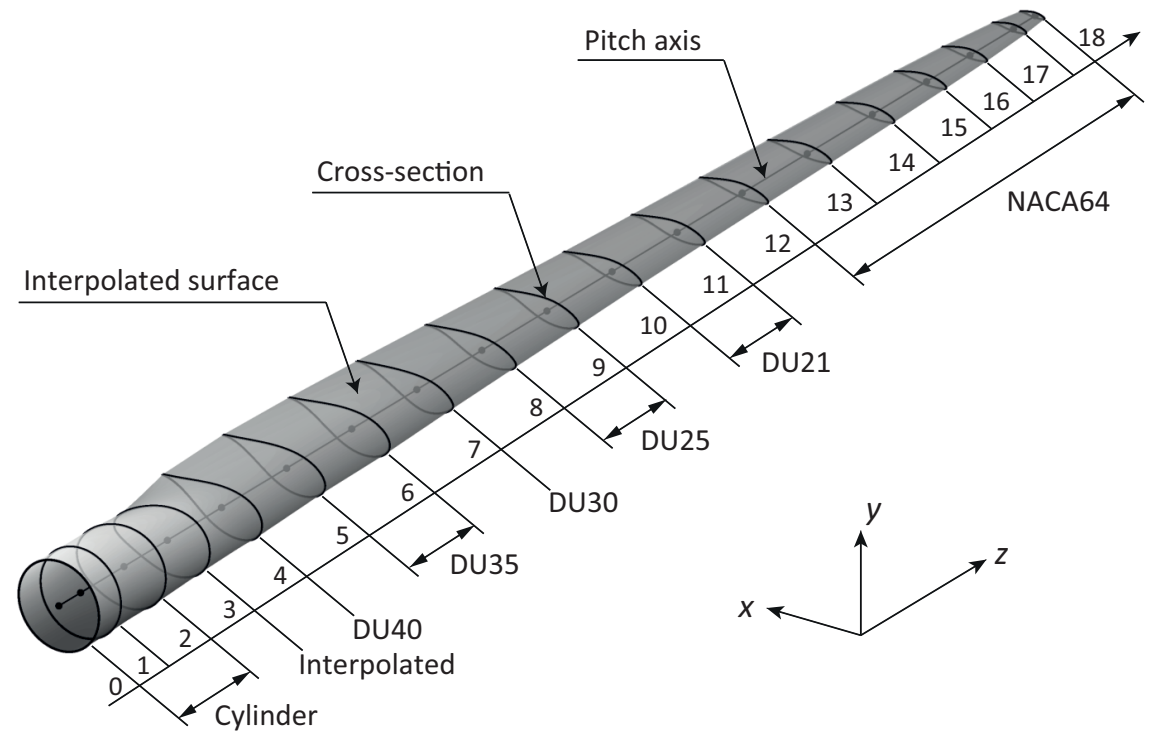

Figure 4.2: Geometry of the NREL 5MW reference wind turbine blade, constructed by 19 cross-sections, each related to a specific airfoil type. The specific cross-sections of the DU and NACA airfoil types are shown in Figure 4.3

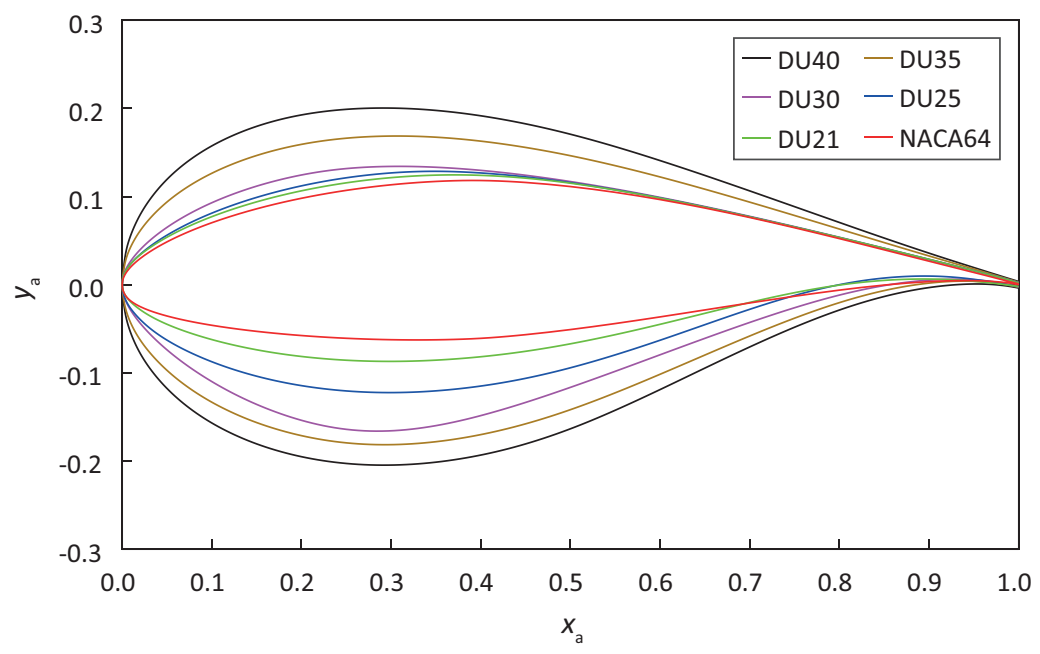

Figure 4.3: Six airfoil types used for constructing the NREL 5MW reference wind turbine blade, where DU and NACA refer to Delft University and National Advisory Committee for Aeronautics, respectively. 


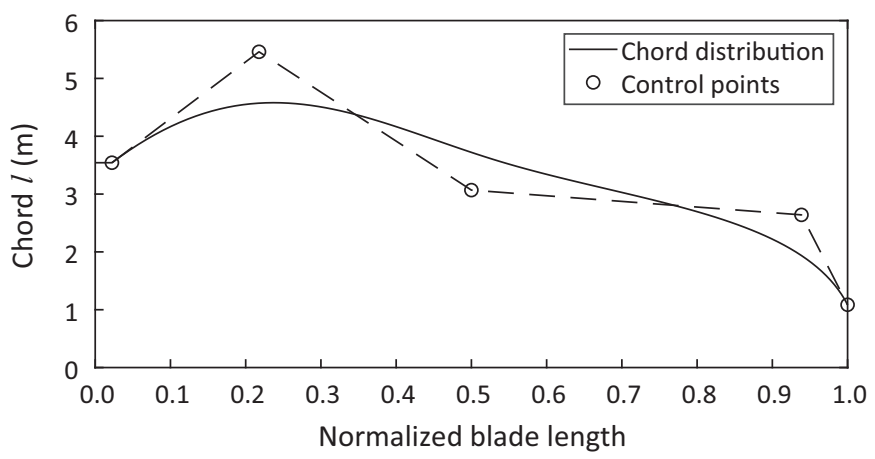

Figure 4.4: Chord distribution along the blade length, described by NURBS with 5 control points. The circles indicate the initial locations of the control points.

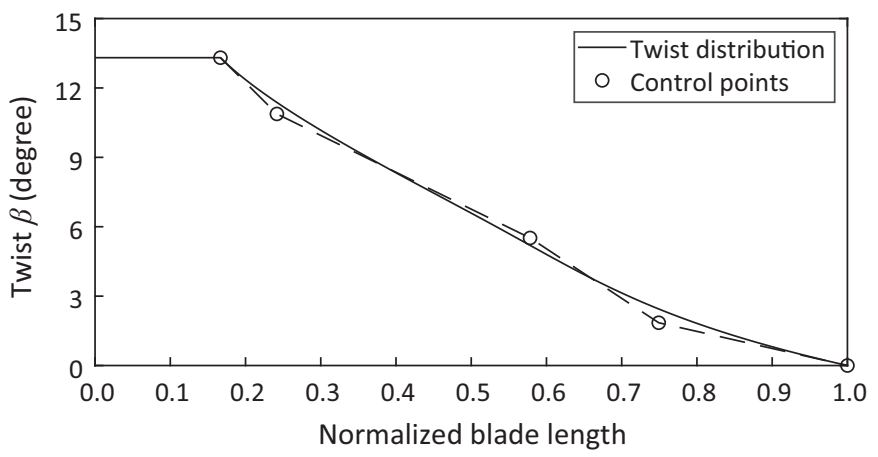

Figure 4.5: Twist distribution along the blade length, described by NURBS with 5 control points. The circles indicate the initial locations of the control points.

Here, $\mathbf{p}_{\mathrm{a}}=\left[x_{\mathrm{a}}, y_{\mathrm{a}}\right]^{\mathrm{T}}$ is an arbitrary point on the normalized airfoil geometry, $\mathbf{o}_{\mathrm{c}}=$ $\left[x_{0}, y_{0}\right]^{\mathrm{T}}$ is the location of the origin of the normalized airfoil geometry, which is chosen to correspond to the blade pitch axis, see Figure 4.2 , with the locations of the pitch axis of the 6 airfoil types depicted in Figure 4.3 taken from [135]. Further, $\mathbf{o}_{\mathrm{b}}=\left[x_{\mathrm{b}}, y_{\mathrm{b}}\right]^{\mathrm{T}}$ represents the location of the origin of the actual cross-section (=blade pitch axis), which for simplicity is defined as $\mathbf{o}_{\mathrm{b}}=[0,0]^{\mathrm{T}}, l$ is the blade chord length of the specific cross-section, as defined in Figure 4.4 and $\mathbf{R}$ is the rotation matrix, given by

$$
\mathbf{R}=\left[\begin{array}{cc}
\cos \beta & -\sin \beta \\
\sin \beta & \cos \beta
\end{array}\right]
$$

with $\beta$ the blade twist angle of the actual cross-section, as defined in Figure 4.5.

The blade cross-sections are discretized by finite elements in order to compute the interior layout of the blade by density-based topology optimization. The mesh 
(a) Normalized airfoil

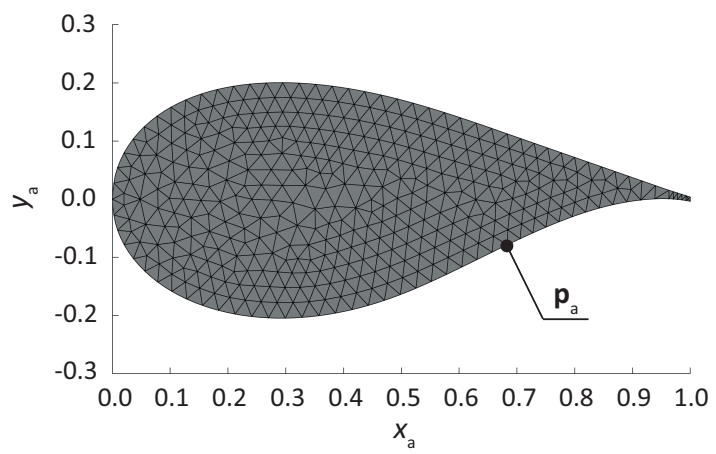

Mapping G

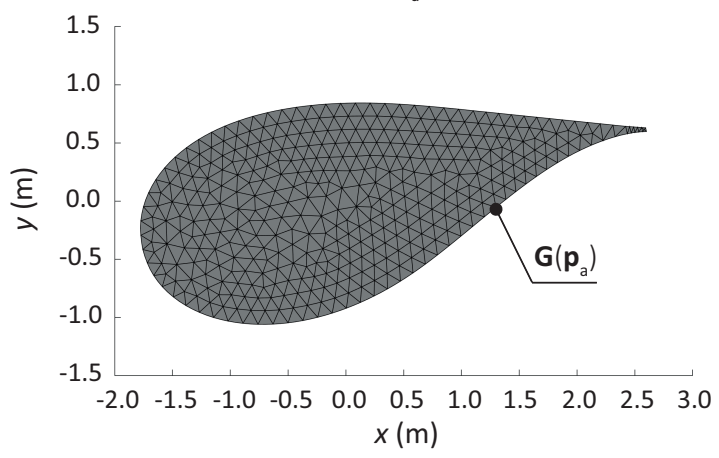

Figure 4.6: Visualization of the geometrical mapping $\mathbf{G}$, given by Eq. 4.6), from a) a normalized airfoil geometry to b) a blade cross-section. The finite element discretization is designated by the solid lines.

of a cross-section is obtained by mapping a predefined finite element mesh for the normalized airfoil using the geometry function $\mathbf{G}$ given by Eq. 4.6). With this procedure, the number of elements, which equals the number of topology design variables $\boldsymbol{\rho}$, is kept fixed for each cross-section during the optimization procedure. Note that cross-sections based on the same airfoil type, such as cross-sections 12 to 18 shown in Figure 4.2, have the same finite element mesh. Accordingly, in order to reduce the computational demand of the optimization procedure, cross-sections with the same finite element mesh are assigned the same set of topology optimization variables $\boldsymbol{\rho}$. Further, the discretization strategy naturally enables the computation of analytical derivatives of the nodal coordinates with respect to the shape design variables $\boldsymbol{a}$, as required for gradient-based optimization.

\subsubsection{Aerostructural analysis}

\subsubsection{Aerodynamic analysis}

For the aerodynamic analysis of the HAWT reference rotor, the BEM method 136 is adopted. This method assumes that the flow around the blade is steady, and that 
sectional stream tubes along the rotor are independent and can be treated separately. Accordingly, a major advantage of the BEM method is its computational efficiency, which makes that it is suitable and widely used for the optimization of large wind turbine blades. However, the assumption of ideal flow conditions leads to an inaccurate prediction of the aerodynamic performance under complex flow patterns. The accuracy of BEM method has been validated by comparing the results with those of high fidelity numerical approaches (e.g., CFD method) and wind tunnel experiments 137-139. These studies indicate that at low and medium wind speeds (below $15 \mathrm{~m} / \mathrm{s}$ ) the results computed by the BEM method correspond well to those of a RANS-CFD approach and wind tunnel experiments, but that at high wind speeds the accuracy reduces as a result of flow separation phenomena [138. Additionally, the accuracy of the BEM method significantly depends on the reliability of the airfoil data in terms of aerodynamic characteristics. To ensure the quality of airfoil data, usually these data are obtained from wind tunnel experiments or high fidelity CFD simulations. Since wind tunnel experiments typically provide the 2D airfoil lift and drag coefficients at a limited number of angles of attack of relatively low value, an extrapolation towards higher angles of attack [140] and a 3D stall-delay correction [141, 142] are required for an appropriate application of the BEM method. For this purpose, some hybrid models have been developed that combine the BEM and CFD methods and use the advantages of both methods [143, 144. In the present work, the airfoil types defining the NREL reference blade (see Section 4.3.1) are kept fixed during optimization, and the airfoil lift and drag coefficients used in the BEM model are determined from wind tunnel experiments with a high angle of attack extrapolation and a 3D rotational correction. Accordingly, the accuracy of the aerodynamic performance of the blade, as predicted by the proposed coupled optimization framework, is expected to be acceptable, although no attempt has been made to verify this in further detail.

The BEM method is reviewed below, and its application towards the aerodynamic analysis of the reference turbine rotor is explained. The cross-section of a rotating turbine blade is illustrated in Figure 4.7. As indicated in this figure, the local flow angle $\phi$ follows from the expression $\tan \phi=U_{\infty}(1-a) /\left(r \Omega\left(1+a^{\prime}\right)\right)$, whereby $r$ is the radial location of the blade cross-section, $\Omega$ is the rotor rotational speed and $U_{\infty}$ represents the free-stream wind speed. Further, $a$ and $a^{\prime}$ are the axial and angular induction factors, respectively, which both are a function of the local flow angle $\phi$, i.e., $a=\hat{a}(\phi)$ and $a^{\prime}=\hat{a}^{\prime}(\phi)$, see [136] for the specific forms. The above expression can be simply reformulated as

$$
f(\phi)=\frac{\sin \phi}{1-a}-\frac{\cos \phi}{\lambda_{r}\left(1+a^{\prime}\right)}=0
$$

where the parameter $\lambda_{r}=r \Omega / U_{\infty}$ is commonly referred to as the local tip-speed ratio. Eq. 4.8 can be solved iteratively for $\phi$ using a one-dimensional root-finding algorithm, such as Brent's method [136]. Once the local inflow angle $\phi$ is determined, the induced aerodynamic normal and tangential forces $F_{\mathrm{n}}$ and $F_{\mathrm{t}}$ acting on the blade cross-section, see Figure 4.7 can be calculated in accordance with the procedure illustrated by the flowchart in Figure 4.8 . The flowchart starts with the computation of the local angle 
of attack $\alpha$ and the local Reynolds number Re, using the value of the local flow angle $\phi$ resulting from Eq. (4.8). As illustrated in Figure 4.7, the angle of attack follows from the expression $\alpha=\phi-\beta$, where $\beta$ is the twist angle, with its distribution along the blade length given in Figure 4.5. The local Reynolds number is computed by inserting the values for the axial and angular induction factors, $a=\hat{a}(\phi)$ and $a^{\prime}=\hat{a}^{\prime}(\phi)$, into the expression for the local inflow velocity, which, in accordance with Figure 4.7, is given by $W=\sqrt{U_{\infty}^{2}(1-a)^{2}+(r \Omega)^{2}\left(1+a^{\prime}\right)^{2}}$. Subsequently, the resulting value of $W$ together with the chord length $l$ is used for computing the local Reynolds number as $R e=\rho W l / \mu$, where $\rho=1.225 \mathrm{~kg} / \mathrm{m}^{3}$ and $\mu=1.812 \times 10^{-5} \mathrm{Ns} / \mathrm{m}^{2}$ represent the density and dynamic viscosity of air, respectively. With the specific angle of attack $\alpha$ and local Reynolds number $R e$ being calculated, the lift coefficient $c_{\mathrm{L}}$ and drag coefficient $c_{\mathrm{D}}$ can be computed from the data tabulated in [133. Figure 4.9 illustrates a specific example of these data, which is related to a DU35 airfoil at a Reynolds number $R e=1 \times 10^{6}$. From the lift and drag coefficients, the lift force $L$ and drag force $D$ can be computed using the expressions $L=c_{\mathrm{L}} \rho W^{2} l / 2$ and $D=c_{\mathrm{D}} \rho W^{2} l / 2$, respectively, see also Figures 4.7 and 4.8. Finally, the forces $F_{\mathrm{n}}$ and $F_{\mathrm{t}}$ normal and tangential to the plane of rotation can be calculated from the lift force $L$ and drag force $D$ via $F_{\mathrm{n}}=L \cos \phi+D \sin \phi$ and $F_{\mathrm{t}}=L \sin \phi-D \cos \phi$, see also Figure 4.7. To apply the aerodynamic forces on the FEM model for the rotor blade, the normal force $F_{\mathrm{n}}$ and tangential force $F_{\mathrm{t}}$ force need to be transferred from the aerodynamic center AC to the reference point $O$ (=pitch axis), as shown in Figure 4.7. This transformation result in the following aerodynamic loads:

$$
\left\{\begin{array}{l}
F_{\mathrm{x}}=F_{\mathrm{t}}, \quad F_{\mathrm{y}}=F_{\mathrm{n}}, \\
M_{\mathrm{z}}=-d\left(F_{\mathrm{t}} \sin \beta+F_{\mathrm{n}} \cos \beta\right),
\end{array}\right.
$$

where $d$ is the distance between the aerodynamic center $\mathrm{AC}$ and the reference point $O$ and $M_{\mathrm{z}}$ is a torsional moment about point $O$. Finally, the forces $F_{\mathrm{x}}, F_{\mathrm{y}}$ and $M_{\mathrm{z}}$ at each blade cross-section are used to construct the global load vector $\mathbf{f}$ used for solving the structural equilibrium of the turbine blade, see Eq. 4.3.

Setting the free-stream wind speed as $U_{\infty}=10 \mathrm{~m} / \mathrm{s}$ and the blade tip-speed ratio as $\lambda_{r}=9$, the distribution of the aerodynamic loads on the blade naturally follows from the normal and tangential forces at the cross-sections along the blade, as depicted in Figure 4.2. For the parameters indicated above the result is depicted in Figure 4.10. Note that the tangential force distribution can be used to calculate the torque generated on the rotor shaft [145, which in turn can be employed to calculate the power coefficient $C_{p}$ of the rotor. As indicated by Eq. 4.1), during the optimization procedure the rotor power coefficient $C_{p}$ is maximized under the given aerodynamic loading conditions. More specific details on the BEM method can be found in [136. 146. In the current work an existing implementation of the BEM method has been used, as provided via the open source software CCBlade, see [132] for background information. 


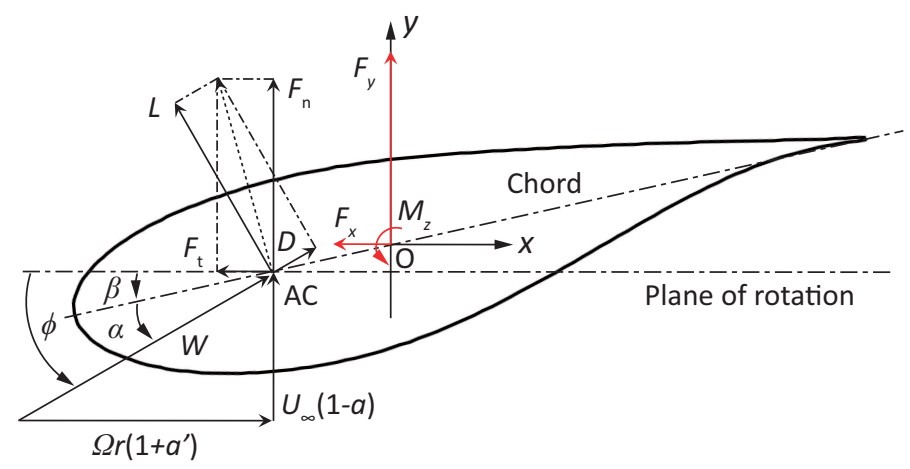

Figure 4.7: Components of the velocity and the aerodynamic load at a cross-section of the rotor blade.

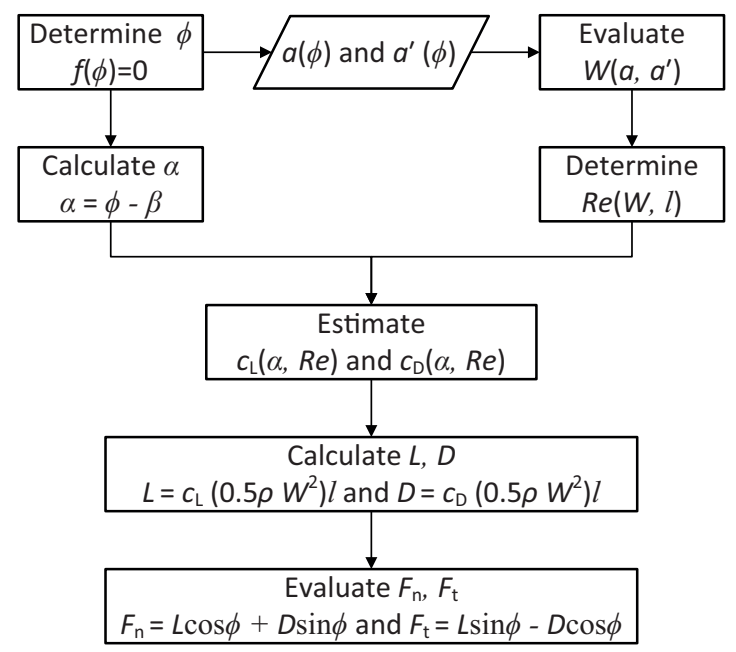

Figure 4.8: Flow chart for the calculation of the aerodynamic forces acting on the blade cross-section.

\subsubsection{Structural analysis}

The structural response of the turbine blade to the aerodynamic loads is calculated by means of an FEM analysis. The turbine blade is discretized as a $2.5 \mathrm{D}$ beam-type structure, which is a model whereby the response of a relatively long and slender structure, such as plotted in Figure 4.11(a), is evaluated by a reduced beam-type FEM configuration shown in Figure 4.11(c), instead of a full 3D FEM configuration sketched in Figure 4.11(b). Specifically, the 2.5D beam-type FEM model simulates the longitudinal direction of the turbine blade by elementary beam elements, for which the properties at specific cross-sections are determined from corresponding $2 \mathrm{D}$ continuum element models, see [131] for more details on this modeling approach. In the present 
work the cross-sections analyzed with a $2 \mathrm{D}$ continuum element model coincide with the cross-sections used for modeling the turbine blade, see Figure 4.2, which allows to straightforwardly impose the aerodynamic loading calculated with the BEM method on the turbine blade.

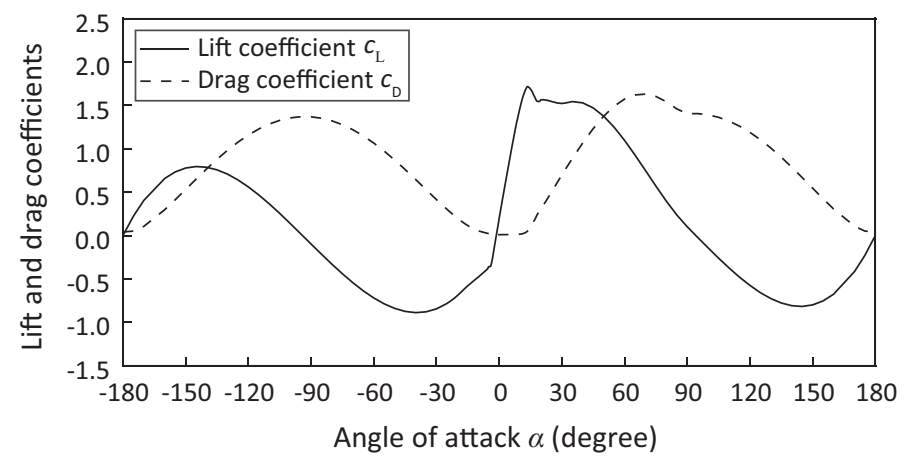

Figure 4.9: Lift and drag coefficients for a DU35 airfoil as a function of the angle of attack, using a Reynold's number $R e=1 \times 10^{6}$.

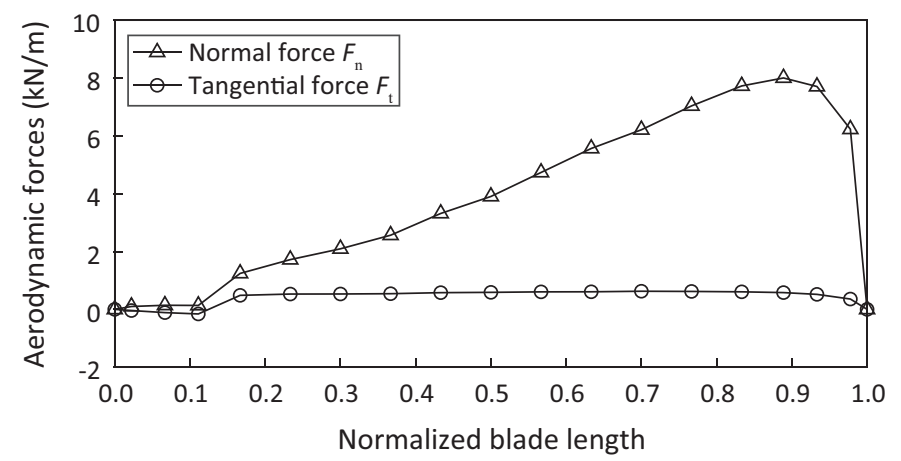

Figure 4.10: Distribution of the normal and tangential aerodynamic forces along the blade length.

The specific stiffness $\mathbf{K}^{s}$ of a blade cross-section is dependent on its outer shape and its internal topology via the shape design variables a and the topology design variables $\boldsymbol{\rho}$. The relative densities representing the topology design variables are evaluated at the individual elements $e$ modeling the $2 \mathrm{D}$ cross-section, $\boldsymbol{\rho}=\boldsymbol{\rho}_{e}$, and determine the Young's modulus of the element in accordance with the SIMP (Solid Isotropic Material with Penalization) approach as 43

$$
E_{e}\left(\rho_{e}\right)=\rho_{e}^{p} E_{0}
$$




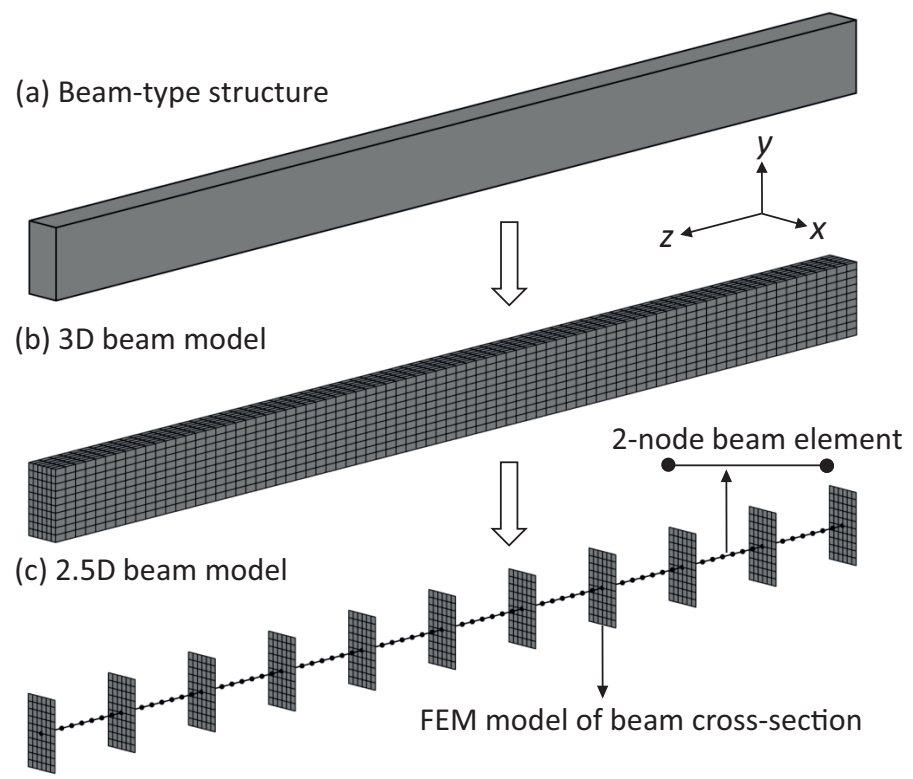

Figure 4.11: FEM discretization. Example of (a) a relative slender beam structure, modeled as (b) a 3D configuration, and (c) a 2.5D configuration.

Here, $p$ is a penalization factor, a typical value being 3 43, and $E_{0}$ is the initial Young's modulus of the material. From the cross-sectional stiffness $\mathbf{K}^{s}$ the beam element stiffness $\mathbf{k}^{b}$ can be constructed, see [131, which, after the element assemblage procedure, provides the global stiffness matrix $\mathbf{K}$ at the structural level. The load vector $\mathbf{f}$ at the structural level is characterized by the aerodynamic forces calculated with the BEM method, as described in Section 4.3.2.1. With the stiffness $\mathbf{K}=\mathbf{K}(\mathbf{a}, \boldsymbol{\rho})$ and load vector $\mathbf{f}=\mathbf{f}(\mathbf{a})$ being characterized, the overall structural compliance $c$ can be calculated by inserting Eq. 4.3 into Eq. 4.2, which results in

$$
c=\mathbf{f}^{\mathrm{T}}(\mathbf{a}) \mathbf{K}^{-1}(\mathbf{a}, \boldsymbol{\rho}) \mathbf{f}(\mathbf{a}),
$$

In order to perform the optimization process in a computationally efficient manner, a gradient-based method is adopted for which the derivatives of the structural compliance $c$ with respect to the shape variables a and topology variables $\boldsymbol{\rho}$ are computed analytically, see Section 4.3 .3 below.

\subsubsection{Sensitivity analysis}

\subsubsection{Shape sensitivity}

As demonstrated in Section 4.3.2.1, the aerodynamic forces $F_{\mathrm{n}}$ and $F_{\mathrm{t}}$ are determined by the geometry of the blade cross-section. In correspondence with Figure 4.6, the 
geometry of a blade cross-section is parameterized by the geometry function $\mathbf{G}$ given by Eq. (4.6), which depends on the chord length $l$ and the twist angle $\beta$ of the blade, see also Eq. (4.7), with their distributions along the blade length presented in Figures 4.4 and 4.5 , respectively. Hence, the derivatives of the aerodynamic forces $\mathbf{F}=\left[F_{\mathrm{n}}, F_{\mathrm{t}}\right]^{\mathrm{T}}$ with respect to the shape design variables a (i.e., the coordinates of the NURBS control points) are calculated by applying the chain rule

$$
\frac{\partial \mathbf{F}}{\partial a_{s}}=\frac{\partial \mathbf{F}}{\partial l} \frac{\partial l}{\partial a_{s}}+\frac{\partial \mathbf{F}}{\partial \beta} \frac{\partial \beta}{\partial a_{s}},
$$

in which $a_{s}$ refers to a specific element in the vector of shape design variables $\mathbf{a}$. The analytical derivatives of the aerodynamic forces with respect to the chord length $\partial \mathbf{F} / \partial l$ and twist angle $\partial \mathbf{F} / \partial \beta$ are provided by the program CCBlade [132]. In addition, analytical expressions for $\partial l / \partial a_{s}$ and $\partial \beta / \partial a_{s}$ can be calculated using NURBS theory, see [131. and references therein. Eq. 4.12 is subsequently substituted into the derivatives of the aerodynamic loads with respect to the shape variables $a_{s}$, which from Eq. 4.9 follow as

$$
\left\{\begin{aligned}
\frac{\partial F_{\mathrm{x}}}{\partial a_{s}}= & \frac{\partial F_{\mathrm{t}}}{\partial a_{s}} \\
\frac{\partial F_{\mathrm{y}}}{\partial a_{s}}= & \frac{\partial F_{\mathrm{n}}}{\partial a_{s}} \\
\frac{\partial M_{\mathrm{z}}}{\partial a_{s}}= & -\frac{\partial d}{\partial a_{s}}\left(F_{\mathrm{t}} \sin \beta+F_{\mathrm{n}} \cos \beta\right) \\
& -d\left(\frac{\partial F_{\mathrm{t}}}{\partial a_{s}} \sin \beta+F_{\mathrm{t}} \cos \beta \frac{\partial \beta}{\partial a_{s}}\right. \\
& \left.+\frac{\partial F_{\mathrm{n}}}{\partial a_{s}} \cos \beta-F_{\mathrm{n}} \sin \beta \frac{\partial \beta}{\partial a_{s}}\right)
\end{aligned}\right.
$$

Note that the derivative $\partial d / \partial a_{s}=0$ when $a_{s}$ is related to the blade twist angle $\beta$, since the distance $d$ between the aerodynamic center $\mathrm{AC}$ and the reference point $O$ is set by the blade chord $l$.

In accordance with the construction of the FEM mesh of a blade cross-section depicted in Figure 4.6, the derivatives of the location of an arbitrary element node $\mathbf{p}=\mathbf{G}\left(\mathbf{p}_{a}\right)=[x, y]^{\mathrm{T}}$ with respect to the element shape design variable $a_{s}$ are obtained from Eq. 4.6 as

$$
\frac{\partial \mathbf{p}}{\partial a_{s}}=\frac{\partial \mathbf{G}\left(\mathbf{p}_{\mathrm{a}}\right)}{\partial a_{s}}=\frac{\partial \mathbf{G}\left(\mathbf{p}_{\mathrm{a}}\right)}{\partial l} \frac{\partial l}{\partial a_{s}}+\frac{\partial \mathbf{G}\left(\mathbf{p}_{\mathrm{a}}\right)}{\partial \beta} \frac{\partial \beta}{\partial a_{s}},
$$

with

$$
\frac{\partial \mathbf{G}\left(\mathbf{p}_{\mathrm{a}}\right)}{\partial l}=\mathbf{R}\left(\mathbf{p}_{\mathrm{a}}-\mathbf{o}_{\mathrm{c}}\right), \frac{\partial \mathbf{G}\left(\mathbf{p}_{\mathrm{a}}\right)}{\partial \beta}=l \frac{\partial \mathbf{R}}{\partial \beta}\left(\mathbf{p}_{\mathrm{a}}-\mathbf{o}_{\mathrm{c}}\right),
$$


where $\mathbf{p}_{\mathrm{a}}$ represents the location of the node in the mesh of the normalized airfoil profile, see Figure 4.6, $\partial \mathbf{R} / \partial \beta$ can be calculated from Eq. (4.7), and $\partial l / \partial a_{s}$ and $\partial \beta / \partial a_{s}$ can be obtained as described below Eq. 4.12. Finally, the derivative of the structural compliance $c$ with respect to to the shape design variable $a_{s}$ follows from Eq. 4.11 as

$$
\frac{\partial c}{\partial a_{s}}=\frac{\partial \mathbf{f}^{\mathrm{T}}}{\partial a_{s}} \mathbf{K}^{-1} \mathbf{f}+\mathbf{f}^{\mathrm{T}} \frac{\partial \mathbf{K}^{-1}}{\partial a_{s}} \mathbf{f}+\mathbf{f}^{\mathrm{T}} \mathbf{K}^{-1} \frac{\partial \mathbf{f}}{\partial a_{s}},
$$

whereby the applied aerodynamic loads $\mathbf{f}$ and their shape sensitivities $\partial \mathbf{f} / \partial a_{s}$ are given by Eqs. 4.9 and 4.13), respectively. Furthermore, the shape sensitivities of $\mathbf{K}^{-1}$ are calculated as $\partial \mathbf{K}^{-1} / \partial a_{s}=-\mathbf{K}^{-1}\left(\partial \mathbf{K} / \partial a_{s}\right) \mathbf{K}^{-1}$, where $\partial \mathbf{K} / \partial a_{s}$ follows the formulation given in 131. The derivatives of the objective function with respect to the shape variables are obtained from Eq. 4.4 as

$$
\frac{\partial f}{\partial a_{s}}=-\frac{\omega C_{\mathrm{p} 0}}{\left(C_{\mathrm{p}}\right)^{2}} \frac{\partial C_{\mathrm{p}}}{\partial a_{s}}+\frac{1-\omega}{c_{0}} \frac{\partial c}{\partial a_{s}},
$$

with

$$
\frac{\partial C_{\mathrm{p}}}{\partial a_{s}}=\frac{\partial C_{\mathrm{p}}}{\partial l} \frac{\partial l}{\partial a_{s}}+\frac{\partial C_{\mathrm{p}}}{\partial \beta} \frac{\partial \beta}{\partial a_{s}},
$$

where $\partial c / \partial a_{s}$ is given by Eq. (4.16), the rotor power coefficient $C_{\mathrm{p}}$ and its derivatives with respect to the chord length, $\partial C_{\mathrm{p}} / \partial l$, and twist, $\partial C_{\mathrm{p}} / \partial \beta$, are obtained from the program CCBlade [132], and $\partial l / \partial a_{s}$ and $\partial \beta / \partial a_{s}$ are derived as described below Eq. 4.12 .

\subsubsection{Topology sensitivity}

The derivative of the structural compliance $c$ with respect to the relative density $\rho_{e}$ in a finite element is obtained from Eqs. 4.2 and 4.3 as

$$
\frac{\partial c}{\partial \rho_{e}}=-\mathbf{u}^{\mathrm{T}} \frac{\partial \mathbf{K}}{\partial \rho_{e}} \mathbf{u}
$$

which uses the assumption that the aerodynamic load vector $\mathbf{f}$ is independent of the element relative densities $\boldsymbol{\rho}$, see also Eq. 4.11. The specific form of $\partial \mathbf{K} / \partial \rho_{e}$ is taken from [131. To alleviate possible checkerboard patterns in the spatial distributions of the element relative densities, a sensitivity filter is introduced 66]

$$
\frac{\partial \hat{c}}{\partial \rho_{e}}=\frac{1}{\rho_{e} \sum_{f=1}^{N} \hat{H}_{f}} \sum_{f=1}^{N} \hat{H}_{f} \rho_{f} \frac{\partial c}{\partial \rho_{f}},
$$




\subsection{Simulation results}

in which $\partial c / \rho_{f}$ is calculated using Eq. 4.19), $\hat{H}_{f}=\max \left(0, r_{\min }-\operatorname{dist}(e, f)\right)$, where $\operatorname{dist}(e, f)$ is the distance between the center of finite element $e$ and the center of element $f$, and $r_{\min }$ is the radius of the circle in which smoothing takes place. Due to the coupling with shape optimization, the element size during the optimization procedure is variable. Consequently, depending on the location and shape of elements, a constant filter radius may cause that multiple elements are filtered together. To avoid this situation, the radius of the filter is defined as $r_{\min }=r_{0} \sqrt{V_{e}}$, where $V_{e}$ is the area of element $e$, and $r_{0}$ is a predefined scaling parameter for the radius, which is set to 1.2. An alternative version of the sensitivity filter, which accounts for varying element sizes, has been presented in 94]. Finally, the derivative of the overall objective function in Eq. (4.5) with respect to the element density $\rho_{e}$ can be formulated as

$$
\frac{\partial f}{\partial \rho_{e}}=\frac{1-\omega}{c_{0}} \frac{\partial \hat{c}}{\partial \rho_{e}},
$$

with $\partial \hat{c} / \partial \rho_{e}$ given by Eq. 4.20.

\subsection{Simulation results}

In order to demonstrate the specific features of the CMSTO framework proposed here, the optimized blade designs following from this approach are compared against the results obtained by two other approaches, namely a separate Aerodynamic Shape Optimization and Structural Topology Optimization (ASO-STO) approach often used in blade design practice, and a pure Structural Topology Optimization (STO) approach. First, the CMSTO approach is applied. In the CMSTO framework, the weighting factor $\omega$ characterizing the objective function, see Eq. (4.1), is varied in order to determine whether the optimization should be biased towards the rotor power coefficient or to the blade structural compliance. For this purpose, five values were selected, which are $\omega=0,0.25,0.50,0.75,0.99$. The maximum value of $\omega$ is set to 0.99 (instead of 1 ), to prevent that the coupled multi-objective optimization model reduces to a pure aerodynamic shape optimization model. Additionally, the maximal material volume fraction $f_{r}$ in Eq. 4.1] is set to 0.125 in accordance with [53, which thus warrants that the total material volume used for the blade should not be more than $12.5 \%$ of the initial blade volume. In the update procedure of the design variables the Sequential Quadratic Programming (SQP) method (implemented in the MATLAB fmincon solver) has been employed, which utilizes the analytical sensitivities presented in Section 4.3.3 Within the current model settings, the computational time required by BEM method for the calculation of the power coefficient and aerodynamic forces of the turbine blade is 8.6 times less than the time needed for the structural analysis.

Figure 4.12 shows the convergence behavior of the CMSTO approach for the normalized blade compliance $c / c_{0}$, the normalized rotor power coefficient $C_{\mathrm{p}} / C_{\mathrm{p} 0}$, and the objective function $f$. Here, $c_{0}$ and $C_{\mathrm{p} 0}$ are the initial values of the structural compliance of the blade and the power coefficient, respectively. The weighting factor used in the CMSTO approach equals $\omega=0.75$. The iterations $g$ and $h$, summed up along 
the horizontal axis, refer to the shape optimization and topology optimization subloops, respectively, see Figure 4.1. In order to clarify the discussion of the results, the convergence behavior following from the alternating shape and topology optimization steps depicted in Figure 4.12 has been divided into three stages, designated as stages I, II and III. The significant variations characterizing stages I and III are shown enlarged in Figures 4.13 and 4.14, respectively. It can be seen from Figure 4.13 that during the initial shape optimization (SO) step of stage I, which is performed in accordance with Eq. (4.4), the normalized power coefficient $C_{\mathrm{p}} / C_{\mathrm{p} 0}$ indeed increases and that the normalized compliance $c / c_{0}$ and the objective function $f$ decrease with an increasing number of iterations. In the subsequent topology optimization (TO) step, $c / c_{0}$ and $f$ continue to decrease with the number of iterations, due to a further optimization of the interior layout of the blade, see Eq. 4.5. The value of $C_{\mathrm{p}} / C_{\mathrm{p} 0}$ remains constant, since the blade outer shape is temporarily kept fixed. Note from stage II in Figure 4.12 that for all three parameters the convergence behavior during this topology optimization step is relatively slow. In the final stage III, the normalized power coefficient $C_{\mathrm{p}} / C_{\mathrm{p} 0}$ again shows a substantial jump during the first shape optimization step, see Figure 4.14. However, after performing two more topology optimization steps and one additional shape optimization step the iterative process has converged by satisfying the stop criterion, $\left|f^{k+1}-f^{k}\right| \leq 1 \mathrm{e}-5$.

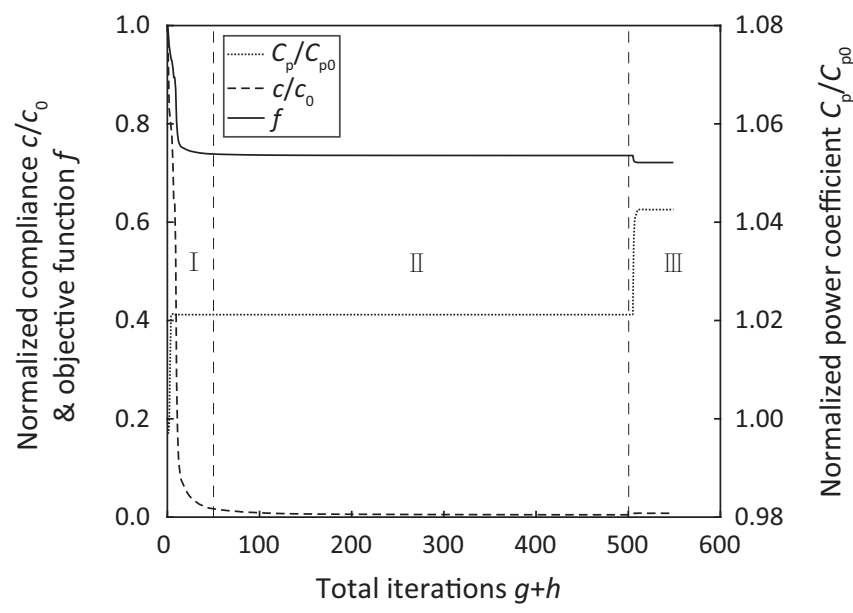

Figure 4.12: Convergence behavior (divided in stages I, II and III) of the normalized compliance $c / c_{0}$, normalized power coefficient $C_{\mathrm{p}} / C_{\mathrm{p} 0}$ and objective function $f$, using the CMSTO approach with $\omega=0.75$.

As illustrated in Figure 4.15, for all five selected weighting factors $\omega$ the CMSTO approach is characterized by a robust convergence behavior of the objective function $f$. For $\omega>0.50$ the total number of iterations appears to increase with increasing value of $\omega$, such that for $\omega=0.99$ the number of iterations is about 5.5 times larger than for $\omega=0.50$ (note that the horizontal axis is scaled differently for $\omega=0.99$ ).

Figure 4.16 illustrates the normalized rotor power coefficient $C_{\mathrm{p}} / C_{\mathrm{p} 0}$ and nor- 


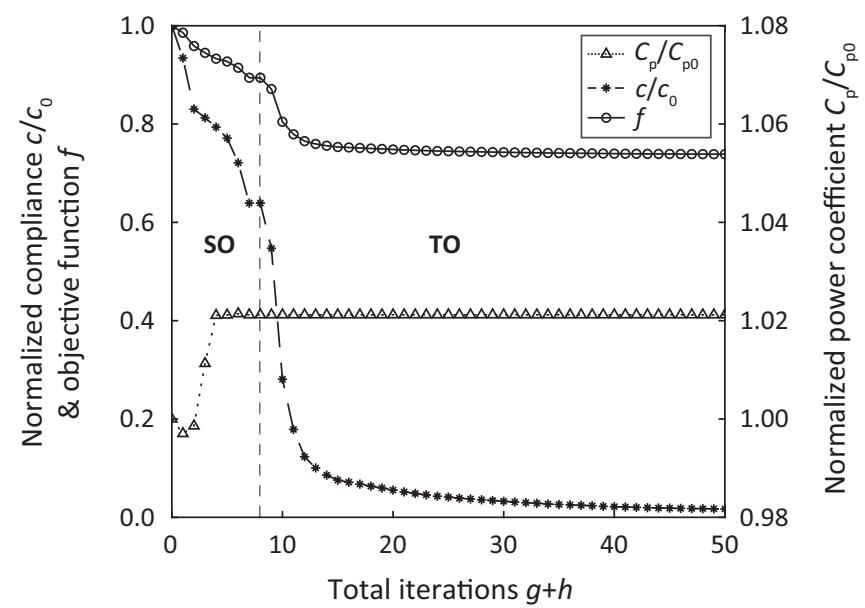

Figure 4.13: Close-up of convergence stage I depicted in Figure 4.12

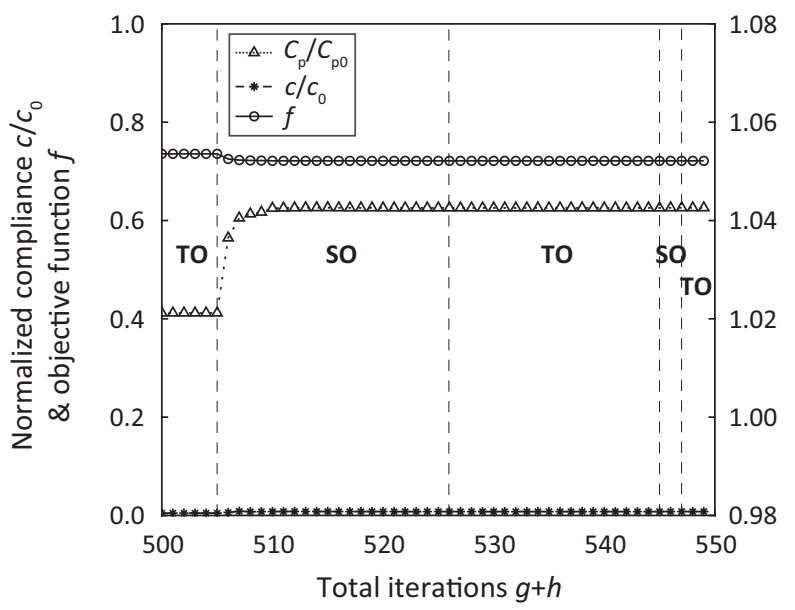

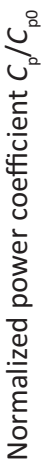

Figure 4.14: Close-up view of convergence stage III depicted in Figure 4.12

malized blade structural compliance $c / c_{0}$ calculated by the CMSTO approach for the 5 selected weight factors, $\omega=0,0.25,0.50,0.75,0.99$, and 5 additional values, $\omega=0.05,0.075,0.10,0.15,0.20$ (circles). The result is compared to that computed by the ASO-STO approach (asterisk) and the STO approach (triangle). In correspondence with [147, the non-dominated points computed by CMSTO approach, which are connected by the dashed line, determine a so-called Approximate Pareto Front (APF). Along the APF, which is represented by weighting factors in the range $0 \leq \omega \leq 0.75$, both the normalized power coefficient $C_{\mathrm{p}} / C_{\mathrm{p} 0}$ and the blade structural compliance $c / c_{0}$ monotonically increase with increasing weighting factor $\omega$. The rotor power co- 


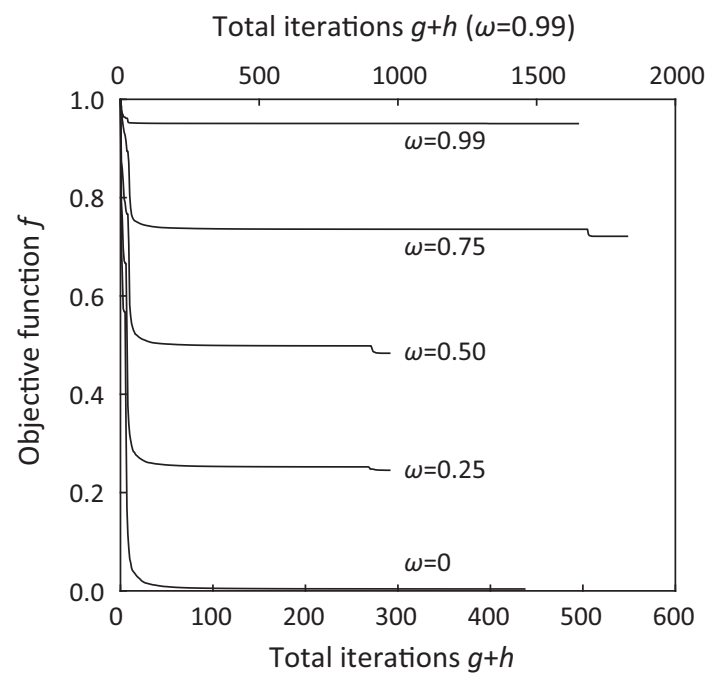

Figure 4.15: Convergence behavior of the objective function $f$, using the CMSTO approach with different weighting factors $\omega$.

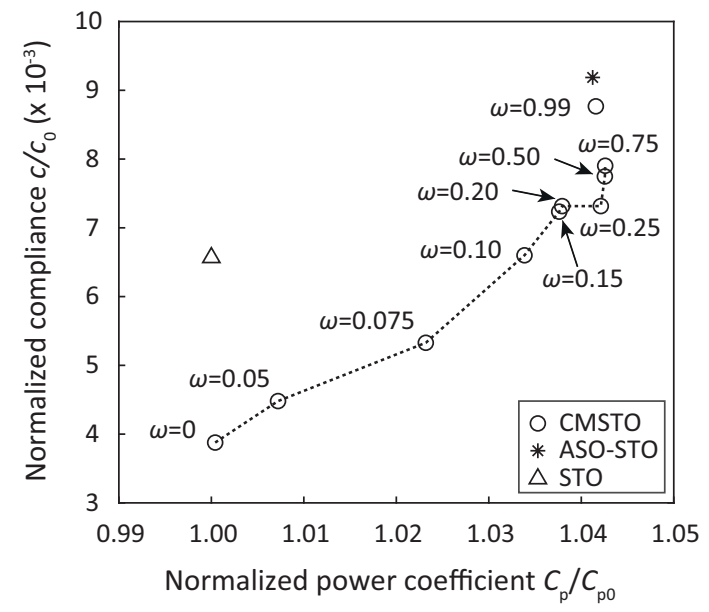

Figure 4.16: Normalized rotor power coefficient $C_{\mathrm{p}} / C_{\mathrm{p} 0}$ and normalized blade structural compliance $c / c_{0}$ of the optimized designs calculated with three different optimization strategies (CMSTO (circles), ASO-STO (asterisk), STO (triangle)). The circles connected by the dashed line represent the Approximate Pareto Front (APF) constructed from the CMSTO results.

efficient of the NREL 5MW wind turbine rotor, used here as a reference model from which the optimization simulations are initiated, equals $C_{\mathrm{p}}=C_{\mathrm{p} 0}=0.459$. As illustrated in Figure 4.16, this value indeed is representative of the two simulations in 


\subsection{Simulation results}

which the power coefficient is left out of consideration in the optimization procedure, i.e., the pure STO approach and the CMSTO approach with $\omega=0$. Note that the normalized structural compliance $c / c_{0}$ is different for these two cases, since in the CMSTO approach the structural compliance is dependent on both the shape and topology design variables, $c=c(\mathbf{a}, \boldsymbol{\rho})$, see Eq.4.1), whereas in the STO approach it only depends on the topology variables, $c=c(\boldsymbol{\rho})$, see Eq. 4.5). By increasing the weighting factor from $\omega=0$ to $\omega=0.75$, the power coefficient grows from 0.459 to 0.479 , which corresponds to an increase of $4 \%$. Hence, it may be concluded that the shape of the NREL 5MW reference blade already is close to optimal from an aerodynamical viewpoint. Note further that an increase of the weighting factor from $\omega=0$ to $\omega=0.75$ leads to an increase of the blade structural compliance of approximately a factor of 2 , which obviously is disadvantageous. In summary, an increase of the power coefficient goes at the expense of an increase in blade compliance (and thus a decrease in blade stiffness), whereby the specific design requirements of the turbine blade determine how these two aspects should be weighted in the optimization process, using a weighting factor within the range of the APF, $0 \leq \omega \leq 0.75$.

The effect of the blade geometry on the aerostructural performance can be examined in more detail by considering the chord and twist distributions of the optimized designs, calculated by CMSTO with $\omega=0,0.05,0.25,0.75$, see Figures 4.17 and 4.18 respectively. It is observed from Figure 4.17 that for a decreasing weighting factor $\omega$ the blade chord length - and also the blade thickness, given the fact that the specific blade cross-section is constant - over a significant part of the blade length becomes larger, which indeed enlarges the overall blade stiffness, see also Figure 4.16. In addition, Figure 4.18 shows that along the first half length of the blade a higher weighting factor $\omega$ leads to a decrease in the blade twist angle $\beta$. Consequently, the angle of attack, $\alpha=\phi-\beta$, of the blade cross-section increases, which - before reaching dynamic stall [124 - results in a higher lift coefficient, and thus in a higher rotor power output, as in agreement with the trend observed in Figure 4.16.

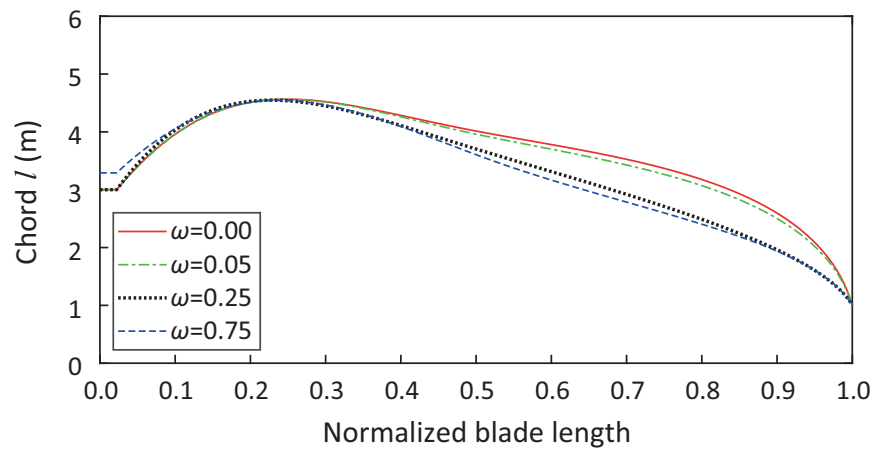

Figure 4.17: Optimized blade chord distribution calculated with the CMSTO approach using different weighting factors $\omega$.

Figure 4.19 shows the optimized outer shape of the blade and the internal topology 


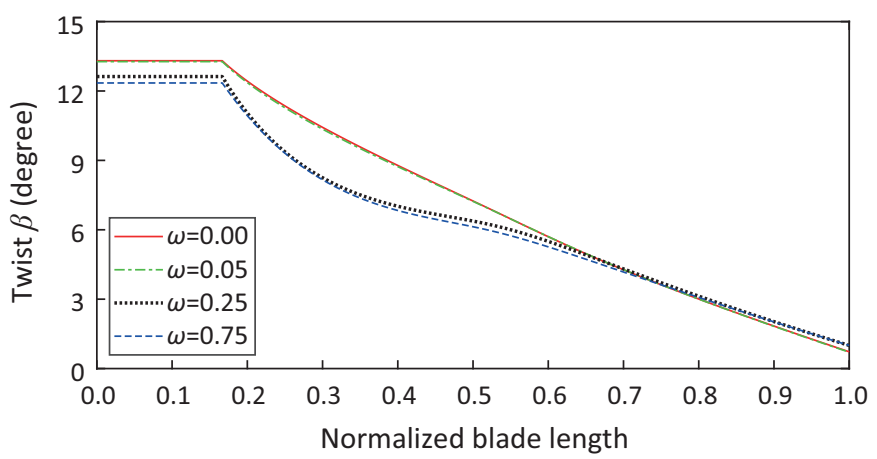

Figure 4.18: Optimized blade twist distribution calculated with the CMSTO approach using different weighting factors $\omega$.

at various blade cross-sections along the blade length, as computed by the CMSTO approach for a weighting factor $\omega=0.75$. It can be observed that for each crosssection two spar caps are found. Furthermore, some cross-sections located between the half length and the tip of the turbine blade are characterized by the presence of two shear webs. Although such a topology indeed bears similarities with conventional blade layout designs [15], the specific size and location of the spar caps and shear webs are optimized in this analysis.

The separate ASO-STO approach often used in blade design practice is also able to optimize both the blade outer shape and the interior layout, but does this in two consecutive steps. Specifically, first the blade outer shape is optimized by maximizing the normalized power coefficient $C_{\mathrm{p}} / C_{\mathrm{p} 0}$ in accordance with Eq. (4.4), whereby the weight factor equals unity, $\omega=1$. Subsequently, with the obtained blade outer shape and corresponding aerodynamic loads, the interior topology of the blade is optimized by minimizing the structural compliance $c / c_{0}$, setting $\omega=0$ in Eq. (4.5). The convergence behavior of the ASO-STO approach is shown in Figure 4.20, by plotting the objectives $C_{\mathrm{p}} / C_{\mathrm{p} 0}$ and $c / c_{0}$ of the ASO and STO steps as a function of the corresponding number of iterations $g$ and $h$, respectively. The figure clearly shows that the number of iterations for the ASO step is almost an order of magnitude lower than for the STO step. The optimized result computed with the ASO-STO approach is depicted in Figure 4.16 using an asterisk symbol. Interestingly, the structural compliance of the blade design following from the ASO-STO approach is higher than that of the CMSTO designs presented, and thus less optimal. On the contrary, the ASOSTO design corresponds to a similarly high power coefficient as found for the CMSTO designs with weighting factors ranging between $0.25 \leq \omega \leq 0.99$; this is obviously due to the fact that in the ASO-STO approach the aerodynamic optimization step is performed first, and that during the subsequent topology optimization step only the interior blade layout is allowed to change.

In Figures 4.21 and 4.22 the chord and twist distributions of the blade designs computed with the CMSTO approach for $\omega=0$ and 0.50 are compared to the distributions 


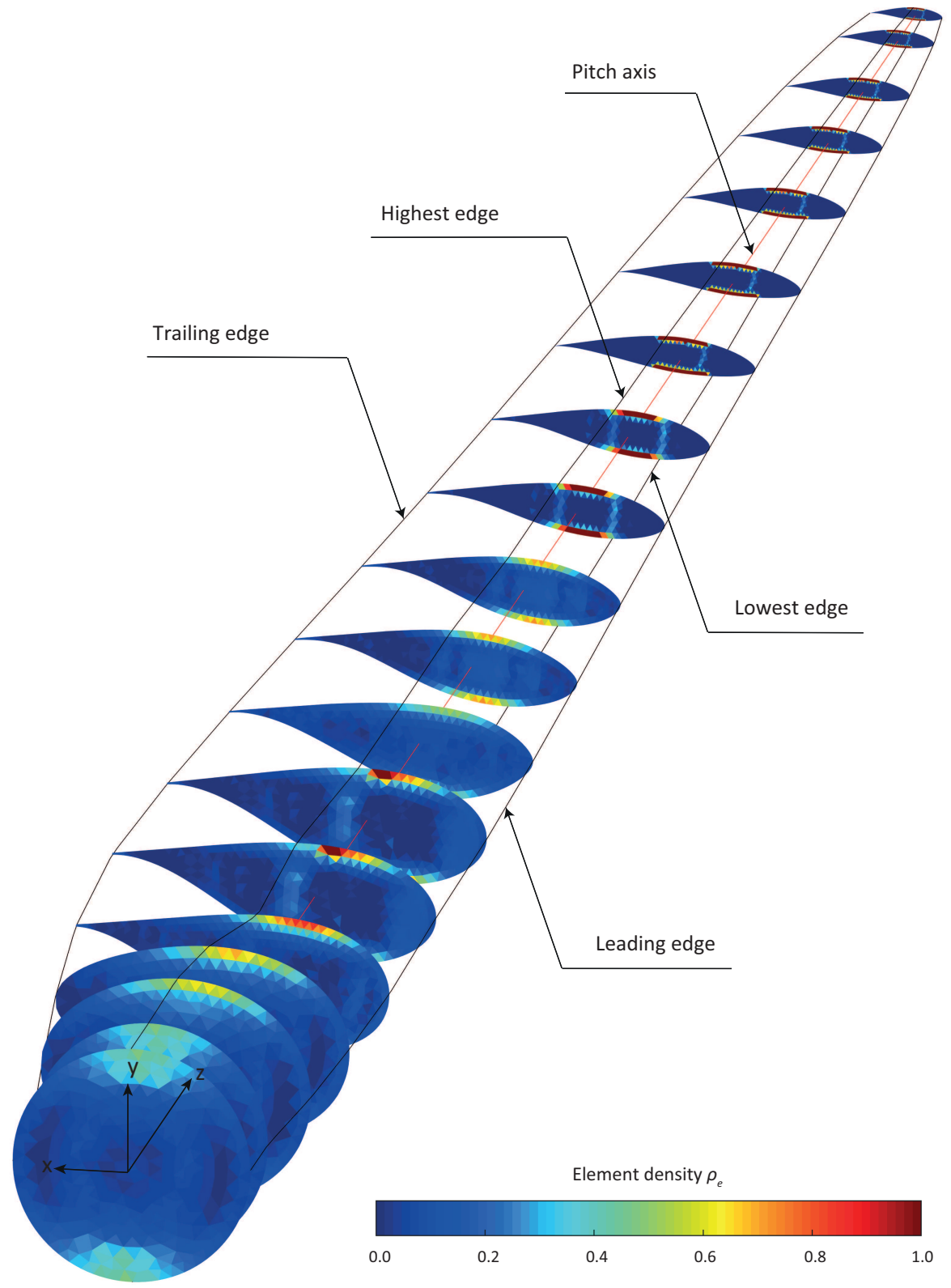

Figure 4.19: Optimized blade geometry and internal topology calculated with CMSTO using a weighting factor $\omega=0.75$. 
calculated by the STO and ASO-STO approaches. The two weighting factors considered here in the CMSTO approach correspond to typical design situations: $\omega=0$ refers to the minimization of the blade structural compliance only, while $\omega=0.5$ reflects that minimizing the blade structural compliance and maximizing the rotor power coefficient are equally important. Note that in the design practice the choice of the weighting factor generally is determined by specific design conditions and requirements. Figure 4.21 shows that for a large part of the blade length the chord length obtained by the CMSTO approach with $\omega=0$ is considerably larger than for the three other cases, which is in correspondence with a significantly higher blade stiffness, see Figure 4.16. In addition, Figure 4.22 indicates that along the first half length of the blade the CMSTO approach with $\omega=0$ and the STO approach result in a larger twist angle than the CMSTO approach with $\omega=0.50$ and the ASO-STO approach, which, as explained above, translates into a lower rotor power coefficient, see Figure 4.16. The above features can be also observed from the optimized geometries of the four cases, see Figure 4.23. The internal topological layouts of the four cases globally look comparable, but differ by the precise locations and dimensions of the spar caps and shear webs.

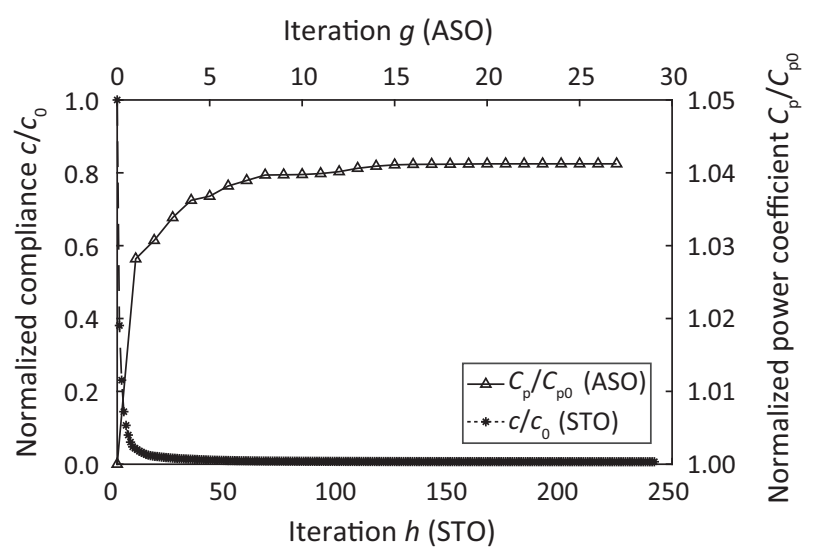

Figure 4.20: Convergence behavior of the normalized power coefficient $C_{\mathrm{p}} / C_{\mathrm{p} 0}$ and normalized compliance $c / c_{0}$, using the separate ASO-STO approach.

The use of a gradient-based optimization approach, together with the Blade Element Momentum method, has made it possible to optimize the three-dimensional rotor blade geometry in a computationally efficient way. Although the use of gradient-free optimization algorithms, such as genetic algorithms [36 41, 59, 60], allows for a more easy numerical implementation and the possibility of finding a global optimum, these algorithms are known to be computationally expensive for density-based topology optimization [148. This is especially the case when considering large structures, such as wind turbine blades, which makes them less suitable for these applications.

Note that in the present work only the aerodynamic loads are considered for the optimization of wind turbine blades. However, in practice, the loading condition of wind 


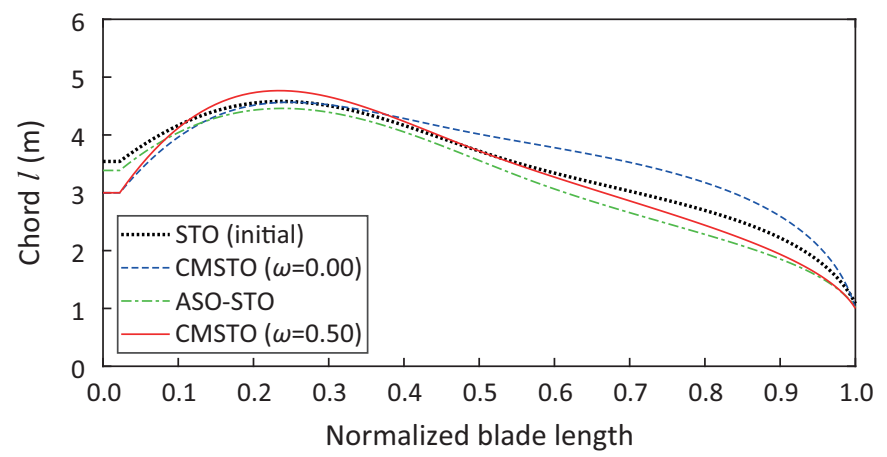

Figure 4.21: Optimized blade chord distribution calculated with the ASO-STO approach and the CMSTO approach with $\omega=0.50$.

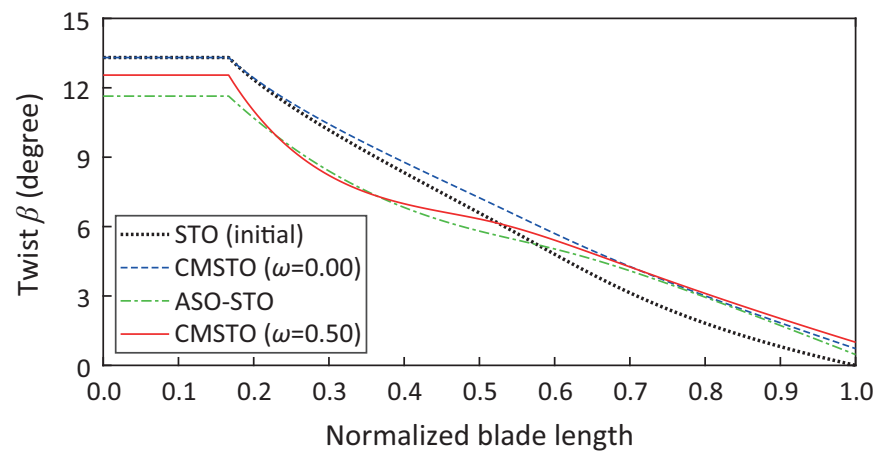

Figure 4.22: Optimized blade twist distribution calculated with the ASO-STO approach and the CMSTO approach with $\omega=0.50$.

turbine blades is rather complicated, and also includes gravitational loads, operational loads, fatigue loads, etc. Furthermore, the aerodynamic loads are calculated in accordance with the specific wind conditions adopted in this work. It may be expected that the optimized blade geometry (both the external shape and interior layout) changes to some extent when multiple loading cases are considered in the coupled optimization framework. Additionally, HAWT blades may undergo large deformations, which makes that the geometrical nonlinear behavior also needs to be considered in the blade design. The 2.5D beam model adopted in this work assumes a linear elastic material behavior, and has been validated by comparing its static response to that of 3D linear elastic finite element models [117. The incorporation of the geometrical nonlinear behavior of the blade into the current coupled optimization framework is considered to be a topic for future work. 

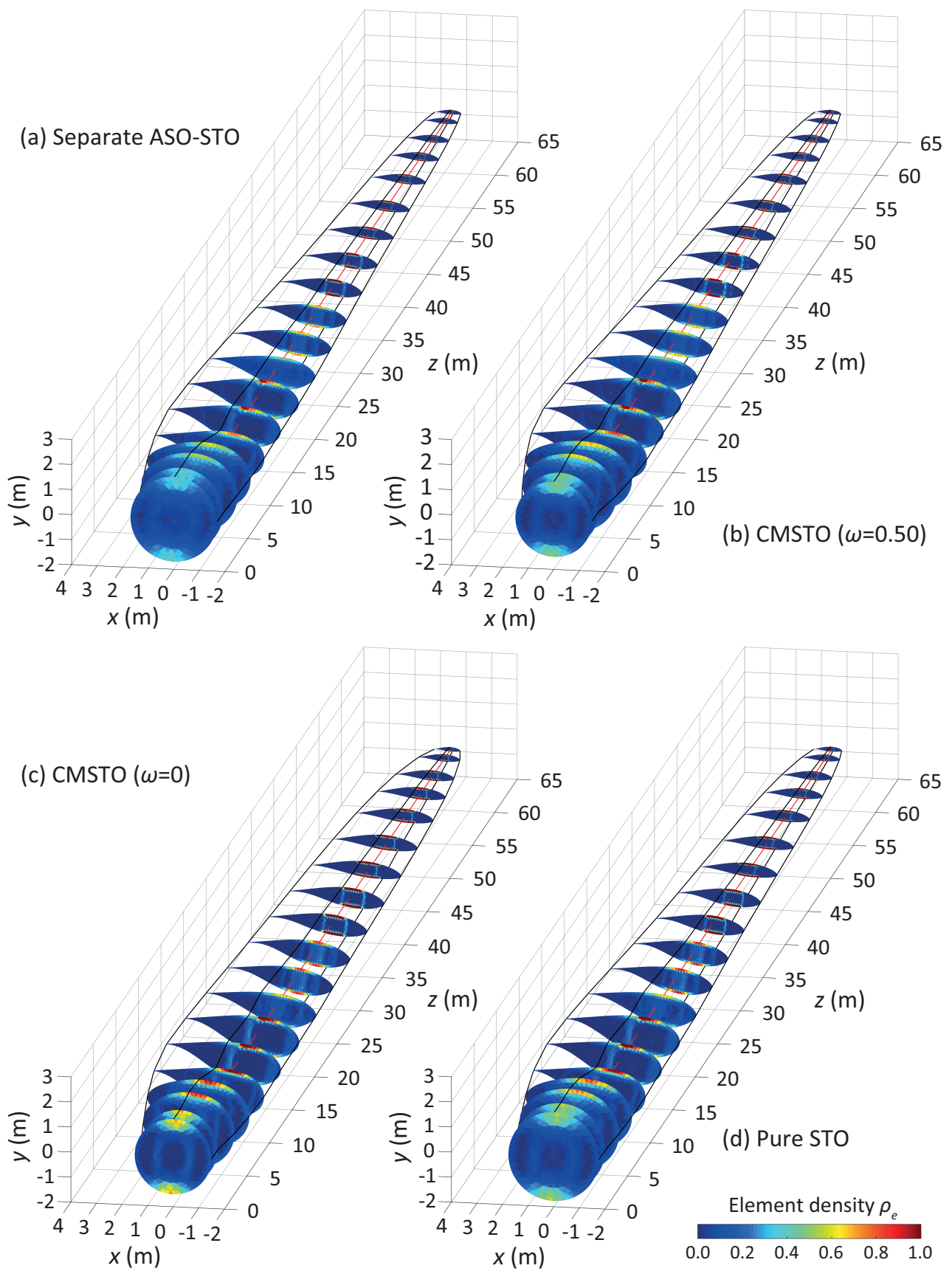

Figure 4.23: Optimized blade geometry and internal topology calculated by (a) the separate ASO-STO approach, (b) the CMSTO approach with $\omega=0.50$, (c) the CMSTO approach with $\omega=0$ and (d) the pure STO approach. 


\subsection{Conclusions}

A coupled multi-objective shape and topology optimization (CMSTO) framework has been proposed for the aerostructural design of HAWT rotor blades. The framework enables to simultaneously optimize the outer shape and the internal structural layout of the turbine blade with respect to aerodynamic and structural requirements. The optimization process is performed on the weighted sum of two design objectives, which are the rotor power coefficient and the structural compliance of the blade. The power coefficient and structural compliance are evaluated in a computationally efficient manner by using so-called BEM and beam FEM models, respectively. The shape design variables are represented by the locations of NURBS control points that govern the blade geometry. The topology design variables are represented by the relative densities assigned to the finite elements modeling the blade cross-sections, in accordance with the SIMP method. The coupled optimization model for a turbine blade is solved in an incremental-iterative fashion, by alternatively performing shape and topology optimization steps, until the solution satisfies the convergence criterion imposed. Here, the computational efficiency of the numerical procedure is warranted through the use of closed-form sensitivities in a gradient-based algorithm for the CMSTO approach.

The specific features of the CMSTO approach are demonstrated by comparing the optimized design computed for a NREL 5MW reference rotor blade with the results obtained from a separate ASO-STO approach and a pure STO approach. The optimization results following from the CMSTO approach for different weighting factors indicate that the overall stiffness of a blade typically decreases with an increase of the rotor power coefficient. The rotor power coefficients of the designs generated by the CMSTO approach with a weighting factor $\omega=0.75$ and the ASO-STO approach are about $4 \%$ larger than that of the reference rotor blade. However, the structural compliance of the blade calculated by the CMSTO approach with $\omega=0.75$ is $16 \%$ lower than that computed by ASO-STO approach. The application of the CMSTO approach with the aerodynamic optimization being switched off leads to a reduction of the blade structural compliance of approximately $41 \%$ compared to a pure STO approach. Such significant improvements clearly demonstrate the benefits of the CMSTO approach in the quest of wind turbines with higher power output and better structural performance.

In order to validate the optimized rotor blade geometries computed in this work, more systematic experimental studies are necessary, both in regards to the shape and topology designs of blade structures. This is a topic for future study. 


\section{Chapter 5}

\section{Comparison of numerical update schemes}

This chapter is based on the journal paper:

Wang, Z., Suiker, A. S. J., Hofmeyer, H., van Hooff, T., Blocken, B., 2019. Optimization of thin-walled beam structures: Monolithic versus staggered solution schemes, Thin-walled Structures, in press, https://doi.org/10.1016/j.tws.2020.107182

This chapter compares the performance of a monolithic and two staggered numerical update schemes for a coupled shape and topology optimization method for thinwalled beam structures. In order to limit the computational time of the optimization method, the thin-walled beam structures are modeled as $2.5 \mathrm{D}$ configurations. In this approach, 1D beam elements are used to simulate the beam response in the longitudinal direction, while the cross-sectional properties of the beam elements are calculated from additional 2D finite element method analyses. The sensitivities with respect to the shape and topology design variables are derived in closed form in order to take advantage of a computationally efficient, gradient-based optimization algorithm. The numerical examples concern basic circular and square thin-walled cantilever beam structures, as well as a more complex, non-prismatic thin-walled beam structure representative of a rotor blade used in a horizontal-axis wind turbine. For each numerical example the computational time and the solution computed by the monolithic and staggered update schemes are compared, which provides clear insight into the numerical efficiency of the solution procedure and the uniqueness of the computational result. Although the three different update schemes for the cases examined result in comparable optimized design concepts, the computational efficiency and the specific minimal compliance value found turn out to be rather sensitive to the algorithmic details of 


\subsection{Introduction}

the numerical update scheme applied. Since the monolithic update scheme typically navigates a larger design space than the two staggered update schemes, for most cases examined it provides the lowest structural compliance. In the specific case whereby shape optimization (virtually) has no influence on the final, optimized beam configuration, the structural compliance computed by the monolithic scheme may relate to a local minimum that is less optimal compared to the value calculated by a staggered update scheme.

\subsection{Introduction}

Thin-walled beam structures are applied as load-bearing components in various engineering applications; illustrative examples are aircraft wings, wind turbine blades and hollow pile foundations. The design of such structures may be challenging, especially when they are subject to complex loading conditions, or when multidisciplinary design requirements need to be taken into account. As an example, a wind turbine must have a high power output and simultaneously needs to have a high structural stiffness and a low structural weight, which are requirements that are typically in conflict with each other [37, 39, 41. Over the past decades, various shape and topology optimization techniques have been applied to meet such design requirements in the most optimal way [42]. In shape optimization, the outer geometry of the beam structure is designed for achieving an improved structural performance [4], which can be accomplished by enlarging the overall stiffness, reducing the local stresses, and/or keeping the static or dynamic response within acceptable bounds [108, 149 151]. In addition, alternative design objectives may be accounted for, such as an enlargement of the lift-drag ratio in the case of aircraft wings, or an increase of the power output in the case of wind turbine systems [152, 153. Conversely, in topology optimization the structural performance is improved by optimally distributing the actual material within a specified, fixed design domain [52. This commonly results in alternative interior layouts, or in local structural reinforcements [53, 71, 115, 154,161]. Due to the nature of topology optimization, the possible solutions for the material distribution are bounded by the specific size and shape of the design domain. This limitation can be mitigated by selecting a design domain with a relatively large size, although this typically leads to a considerable increase in computational time. Another drawback of a fixed design domain is that it impedes the optimization of aeroelastic structures, such as aircraft wings and wind turbine blades. Since the aerodynamic loading is characterized by the size and shape of the design domain, the design domain of aeroelastic structures needs to be variable in order to optimize their aerodynamic performance.

Recently, a coupled shape and topology optimization method has been proposed in order to overcome the above limitations of a fixed design domain 112. This method has been subsequently applied in 131 to optimize the outer shape and internal layout of beam-type structures. The beam structure is discretized using the Finite Element Method (FEM), whereby the outer shape of the beam is described parametrically via Non-Uniform Rational B-Splines (NURBS) [89]. The use of NURBS ensures a highly accurate description of the shape boundaries by means of smooth basis functions with 
compact support, and enables an efficient control of the design domain with only a few control points. The internal layout of the beam structure is described by the relative densities of the finite elements modeling the beam cross-sections, in accordance with the Solid Isotropic Material with Penalization (SIMP) approach [43. The computational time of the optimization procedure is kept manageable by i) using an economical, gradient-based optimization algorithm, and ii) modeling the three-dimensional beam structure in accordance with an efficient $2.5 \mathrm{D}$ beam formulation originally proposed in 113 . In this formulation, 1D beam elements are employed to simulate the beam response in the longitudinal direction, whereby the cross-sectional properties of the beam elements are determined from additional 2D FEM analyses, see also [117. The practical applicability of the coupled shape and topology optimization method has been demonstrated recently in an aerostructural design analysis of a Horizontal-Axis Wind Turbine (HAWT) rotor blade [119. The optimization study showed that the incorporation of coupling effects between the aerodynamical and structural behavior of the turbine blade may result in non-standard designs characterized by both a high power output and a large structural stiffness.

The coupled optimization model proposed in [112] uses a staggered numerical update scheme, in which the shape and topology design variables are incrementally updated in a sequential fashion. It has been demonstrated that this update scheme is numerically robust, and leads to meaningful and practically useful results [119, 131]. Alternatively, the coupled optimization model may use a monolithic numerical update scheme, whereby the shape and topology design variables are incrementally updated in a concurrent fashion. Since monolithic and staggered update schemes follow different search paths in the design space, the numerical efficiency and the solution obtained by these update schemes in principle is different. Obviously, this sensitivity needs to be explored and well understood in order to develop a solid confidence in the results calculated by the coupled shape and topology optimization method. Accordingly, in the present communication the optimization results computed from monolithic and staggered update schemes are compared for various thin-walled beam structures. The numerical examples concern basic circular and square thin-walled cantilever beam structures, as well as a more complex, non-prismatic thin-walled beam structure representative of a rotor blade used in a horizontal-axis wind turbine. The aerodynamic loading applied on the rotor blade is dependent on the shape of the design domain, whereby the aerodynamic performance is warranted through the formulation of a separate constraint condition. For each boundary value problem analyzed the computational time and the solution computed by the monolithic and staggered update schemes are compared, which should provide clear insight into the numerical efficiency of the solution procedure and the uniqueness of the computational result.

This chapter is organized as follows. Section 5.2 reviews the geometrical description of thin-walled beam structures, and introduces the shape and topology design variables. In Section 5.3 the main features of the coupled shape and topology optimization method are presented. The monolithic and staggered update schemes are outlined, and the analytical shape and topology sensitivities used in the gradient-based solution method are provided. Section 5.4 discusses the optimization results computed for circular and square thin-walled cantilever beams and an HAWT rotor blade. The 


\subsection{Geometrical description and design variables}

main conclusions of the comparison study are presented in Section 5.5 .

\subsection{Geometrical description and design variables}

The formulation of the coupled shape and topology optimization approach starts with a parametric description of the thin-walled beam geometry in terms of appropriate design variables. In order to keep the computational time of the approach manageable, a three-dimensional beam structure is described by means of an efficient 2.5D beam formulation originally proposed in [113. In this method, 1D beam elements simulating the beam response in the longitudinal direction are combined with two-dimensional cross-sectional design units defining the beam geometry at given locations along the longitudinal beam axis. In the present work, the initial beam cross-section is assumed to be thin-walled and hollow, i.e., a circular or a square tube, or a hollow airfoil defining the circumference of a rotor blade of a HAWT. The variation of the crosssectional properties in the longitudinal beam direction follows from scaling the crosssectional dimension by a factor $l$, and rotating the cross-section by a twist angle $\beta$. Adopting a Cartesian coordinate system, whereby the $x$ - and $y$-coordinates of crosssectional points are normalized by the scaling factor $l$, i.e., $x_{\mathrm{a}}=x / l$ and $y_{\mathrm{a}}=y / l$, this geometrical mapping, which has been visualized in Figure 5.1 for an airfoil crosssectional design unit, can be expressed via the geometry function

$$
\mathbf{G}\left(\mathbf{p}_{\mathrm{a}}\right)=l \mathbf{R}\left(\mathbf{p}_{\mathrm{a}}-\mathbf{o}_{\mathrm{c}}\right)+\mathbf{o}_{\mathrm{b}}
$$

(a) Airfoil design unit
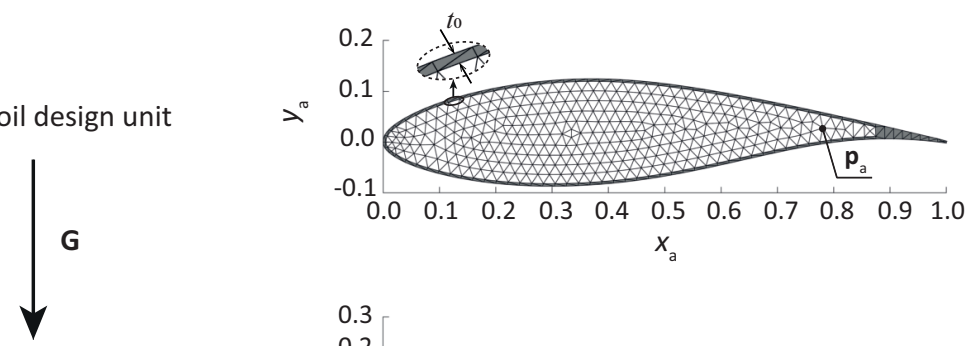

(b) Blade cross-section

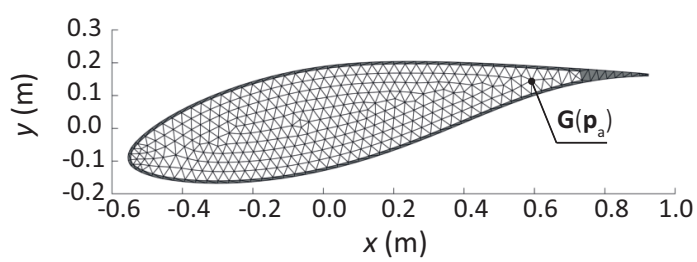

Fig. 5.1. Visualization of the geometrical mapping G presented by Eq. (5.1) from (a) an airfoil design unit (defined in $x_{\mathrm{a}}-y_{\mathrm{a}}$ coordinate system) to (b) a blade cross-section (defined in the $x-y$ coordinate system). The finite element mesh is indicated by the solid lines.

Here, $\mathbf{p}_{\mathrm{a}}=\left[x_{\mathrm{a}}, y_{\mathrm{a}}\right]^{\mathrm{T}}$ refers to an arbitrary, normalized location within the airfoil design unit, $\mathbf{o}_{\mathrm{c}}=\left[x_{0}, y_{0}\right]^{\mathrm{T}}$ is the location of the normalized reference point in the airfoil design 
unit, $\mathbf{o}_{\mathrm{b}}=\left[x_{\mathrm{b}}, y_{\mathrm{b}}\right]^{\mathrm{T}}$ is the actual location of the reference point (which corresponds in Figure 5.1 (b) to the pitch axis $[0,0]^{\mathrm{T}}$ of the airfoil), $l$ is the scaling factor, and $\mathbf{R}$ is the rotation matrix, expressed in terms of the twist angle $\beta$, i.e.,

$$
\mathbf{R}=\left[\begin{array}{cc}
\cos \beta & -\sin \beta \\
\sin \beta & \cos \beta
\end{array}\right]
$$

(a) Chord length distribution

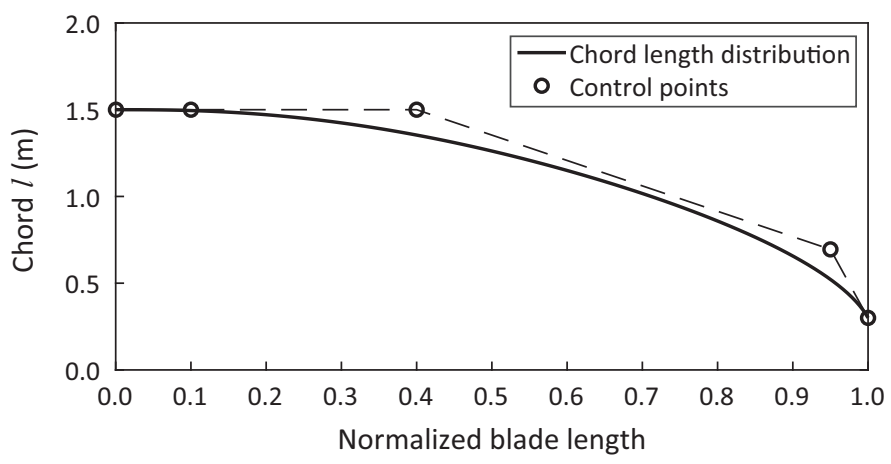

(b) Twist angle distribution

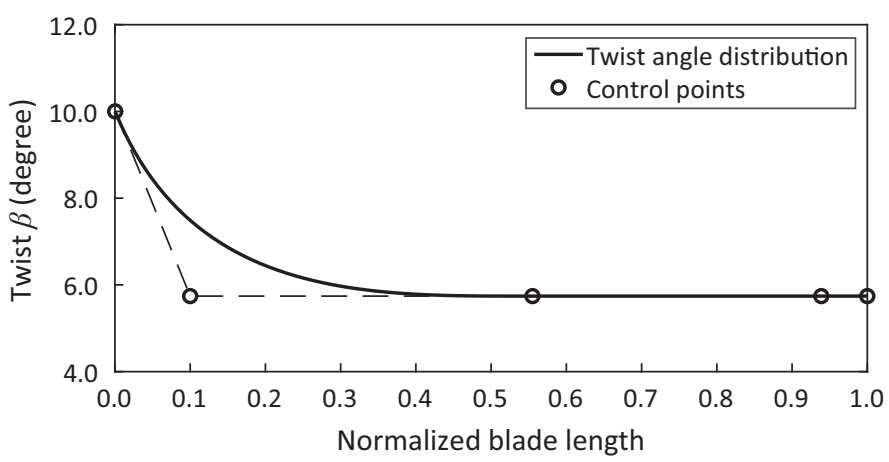

Fig. 5.2. Geometry parameterization by using NURBS with 5 control points. The circles indicate the initial locations of the control points. (a) Distribution of chord length $l$ along the blade length, and (b) distribution of twist angle $\beta$ along the blade length.

The distributions of the design variables $l$ and $\beta$ in the longitudinal direction of the beam structure are represented by NURBS curves equipped with 5 control points. Figure 5.2 shows an example of these distribution functions for a specific airfoil crosssection, whereby the coordinates of the NURBS control points act as the shape design variables a that parameterize the geometry during the optimization procedure. More background information on the theory of NURBS and their implementation for shape optimization problems can be found in [89, 131]. After the initial beam geometry is 


\subsection{Geometrical description and design variables}

parameterized from the definition of appropriate cross-sectional design units and the corresponding distribution functions for $l$ and $\beta$, specific locations need to be selected along the longitudinal beam direction at which the cross-sectional characteristics under the applied loading will be computed from two-dimensional FEM analyses 117, 131. As an example, Figure 5.3 (a) shows an HAWT rotor blade constructed by 11 different blade cross-sections, whereby the geometrical properties in between two adjacent crosssections follow from a linear interpolation. For simplicity, the 11 cross-sections are defined from a single design unit, i.e., the airfoil type sketched in the same figure; note, however, that the parameterization of (more complex) turbine blades applied in HAWTs requires the use of multiple design units [119].

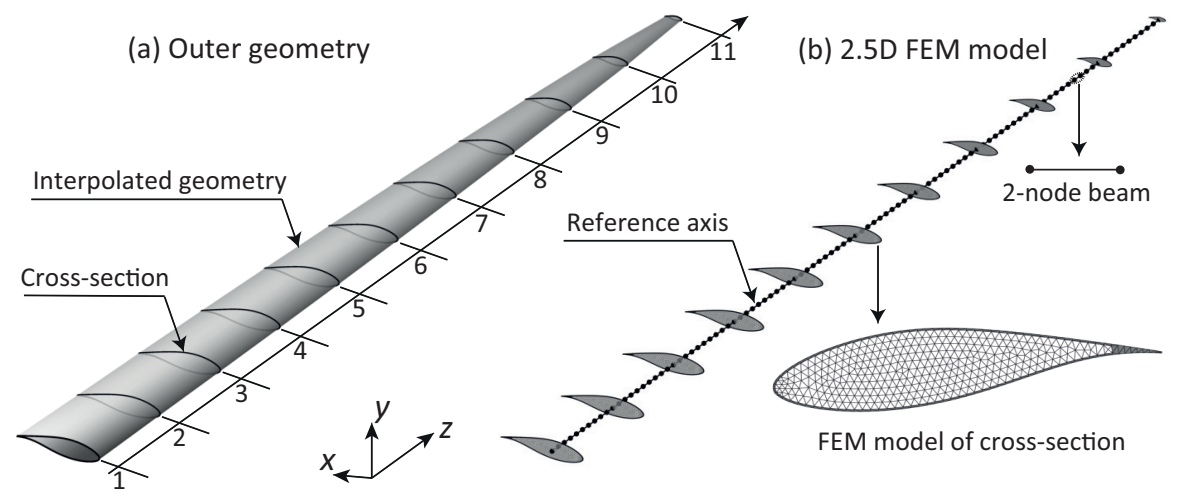

Fig. 5.3. An HAWT rotor blade constructed by 11 different cross-sections. The crosssections are characterized by the shape design variables $l$ and $\beta$ following from the distribution functions shown in Figure 5.2. (a) Outer geometry, and (b) 2.5D FEM model.

In the present work the outer shape of the thin-walled beam structure is optimized together with its internal topology, whereby the topology optimization is performed in accordance with the SIMP approach [43]. The topology of the cross-sectional design unit follows from optimizing the relative densities $\boldsymbol{\rho}$ of the finite elements, which represent the topology design variables. The values of the relative densities range between zero (a void) and unity (a solid), e.g., for the initial, hollow core airfoil shown in Figure 5.1(a), the finite elements modeling the outer wall of thickness $t_{0}$ are kept fixed at unity density (colored dark grey) and the interior finite elements modeling the hollow space are assigned an initial density value of zero (colored white). Accordingly, during the optimization procedure the outer wall remains solid and only the relative densities of the interior elements are optimized. The mesh generation of the beam cross-section is carried out by projecting the auxiliary mesh of the design unit onto the actual cross-section via the geometry function $\mathbf{G}$ given by Eq. (5.1), see also Figure 5.1. Accordingly, an explicit relation between the finite element mesh and the shape design parameters $l$ and $\beta$ can be established, from which analytical sensitivities can be computed that facilitate a computationally efficient, gradient-based solution procedure of the coupled optimization problem [119, 131]. 


\subsection{Coupled shape and topology optimization ap- proach}

\subsubsection{General framework}

In the coupled optimization approach the structural compliance $c$ of the beam configuration is optimized for the shape design variables a and topology design variables $\boldsymbol{\rho}$ via the formulation

$$
\begin{array}{cll}
\min _{\mathbf{a}, \boldsymbol{\rho}} & f(\mathbf{a}, \boldsymbol{\rho})=\frac{c(\mathbf{a}, \boldsymbol{\rho})}{c_{0}}, & \\
\text { subject to } & \frac{V(\mathbf{a}, \boldsymbol{\rho})}{V_{\max }} \leq 1, & \\
& \mathbf{A} \mathbf{a} \leq \mathbf{b}, & \\
& \mathbf{h}_{g}(\mathbf{a}) \leq \mathbf{0}, & \\
& & \\
\text { with } & 0 \leq \frac{a_{s}-l_{s}}{u_{s}-l_{s}} \leq 1, \ldots, G, \\
& 0 \leq \rho_{\min } \leq \rho_{e} \leq 1, & e=1, \ldots, E,
\end{array}
$$

Here, $a_{s}$ is a component of the vector of shape design variables $\mathbf{a}$, with the lower and upper bounds given by $l_{s}$ and $u_{s}$, respectively, and the total number of components equal to $S$. The vector of topology design variables $\boldsymbol{\rho}$ contains the relative element densities $\rho_{e}$, whereby a small, minimal density value $\rho_{\min }$ is defined to avoid a singular stiffness matrix, and the total number of components is set by $E$. Further, $V$ is the material volume, which, by means of an inequality constraint, cannot exceed the maximal material volume $V_{\max }$ specified a priori. The matrix $\mathbf{A}$ and vector $\mathbf{b}$ define the linear inequality constraints for the shape design variables $\mathbf{a}$, and $\mathbf{h}_{g}$ includes the non-linear inequality constraints for $\mathbf{a}$, with the total number of non-linear constraints given by $G$. Note that in Eq. (5.3) the shape design variable $a_{s}$ has been normalized in order to range between zero and one, by which the scale difference in the ranges for the shape and topology design variables vanishes. In the expression for the objective function $f$ the structural compliance $c$ is normalized by its initial value $c_{0}$, whereby $c$ follows from the FEM model as

$$
c=\mathbf{f}^{\mathrm{T}} \mathbf{u}
$$

with $\mathbf{f}=\mathbf{f}(\mathbf{a})$ the global force vector and $\mathbf{u}=\mathbf{u}(\mathbf{a}, \boldsymbol{\rho})$ the global displacement vector. The force vector $\mathbf{f}$, which may depend on the shape design variables $\mathbf{a}$ in order to account for shape-dependent, aerodynamic loads, is related to the displacement vector u via

$$
\mathbf{K u}=\mathbf{f}
$$




\subsection{Coupled shape and topology optimization approach}

with $\mathbf{K}=\mathbf{K}(\mathbf{a}, \boldsymbol{\rho})$ the global stiffness matrix. In the $2.5 \mathrm{D}$ beam formulation the global stiffness matrix assembles the stiffness matrices of the beam elements, for which the cross-sectional response is determined from 2D FEM analyses, see [113, 117, 131] for more details. The Young's modulus $E_{e}$ of a cross-sectional finite element is determined by

$$
E_{e}=\rho_{e}^{p} E_{0},
$$

where $\rho_{e}$ is the relative density of element $e, p$ is a penalization factor, a typical value being 3 [43], and $E_{0}$ is the initial Young's modulus of the material. Note that via Eq. 5.6 the global stiffness matrix $\mathbf{K}$ becomes dependent on the topology design variables $\boldsymbol{\rho}$. The initial values $\boldsymbol{\rho}_{0}$ of the topology design variables in the cross-sectional design units are defined by

$$
\rho_{0}=\frac{V_{\max }-V_{0}^{\mathrm{w}}}{V_{0}^{\mathrm{i}}}
$$

where $V_{\max }$ is the maximal material volume, see also Eq. 5.3 , and $V_{0}^{\mathrm{w}}$ and $V_{0}^{\mathrm{i}}$ are the initial volumes of the wall and interior of the thin-walled beam, respectively.

In Eq. (5.5), the dependency of the stiffness matrix $\mathbf{K}$ on the shape design variables a enters through the variation of the cross-sectional design unit along the longitudinal beam direction, as quantified by the locations of the NURBS control points defining the actual chord and twist distributions in the longitudinal direction, see Figure 5.2 .

\subsubsection{Monolithic and staggered update schemes}

The coupled optimization formulation given by Eq. (5.3) may be solved by either applying a monolithic update scheme or a staggered update scheme. In the monolithic update scheme the shape and topology design variables a and $\boldsymbol{\rho}$ are incrementally updated in a concurrent fashion, as illustrated by the flowchart presented in Figure 5.4. In the discussion of the results, this approach will be referred to as Concurrent Optimization (CO). The monolithic scheme starts with i) the description of the geometry of the structure (as explained in Section 5.2), followed by ii) performing a structural FEM analysis, iii) a sensitivity analysis, and finally iv) the update of the design variables. The last two stages of the numerical update scheme will be explained in more detail in Sections 5.3.3 and 5.3.4 During each increment, the combination of stages ii) to iv) is repeated iteratively, until the stop criterion is reached and the numerical process converges. Alternatively, a staggered update scheme can be applied, whereby the design variables a and $\boldsymbol{\rho}$ are incrementally updated in a sequential fashion, in accordance with the flowchart presented in Figure 5.5. Again, the update procedure starts with the geometry description, after which the individual shape and topology optimization steps composed of the above stages ii) to iv) are performed alternately, until the stop criteria for both optimization steps are satisfied. The solution scheme depicted in Figure 5.5 starts with a topology optimization step, and, as such, is referred to as Coupled Topology Shape Optimization (CTSO). Alternatively, 
it may start with a shape optimization step, and then is referred to as Coupled Shape Topology Optimization (CSTO).

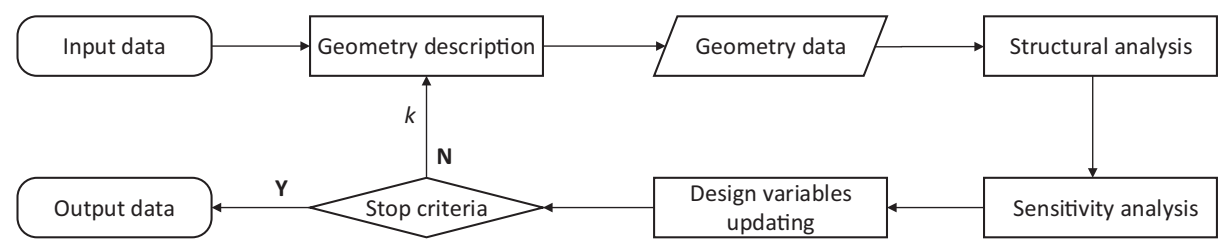

Fig. 5.4. Flowchart of the monolithic update scheme for coupled optimization, with iteration $k$ referring to the optimization loop.

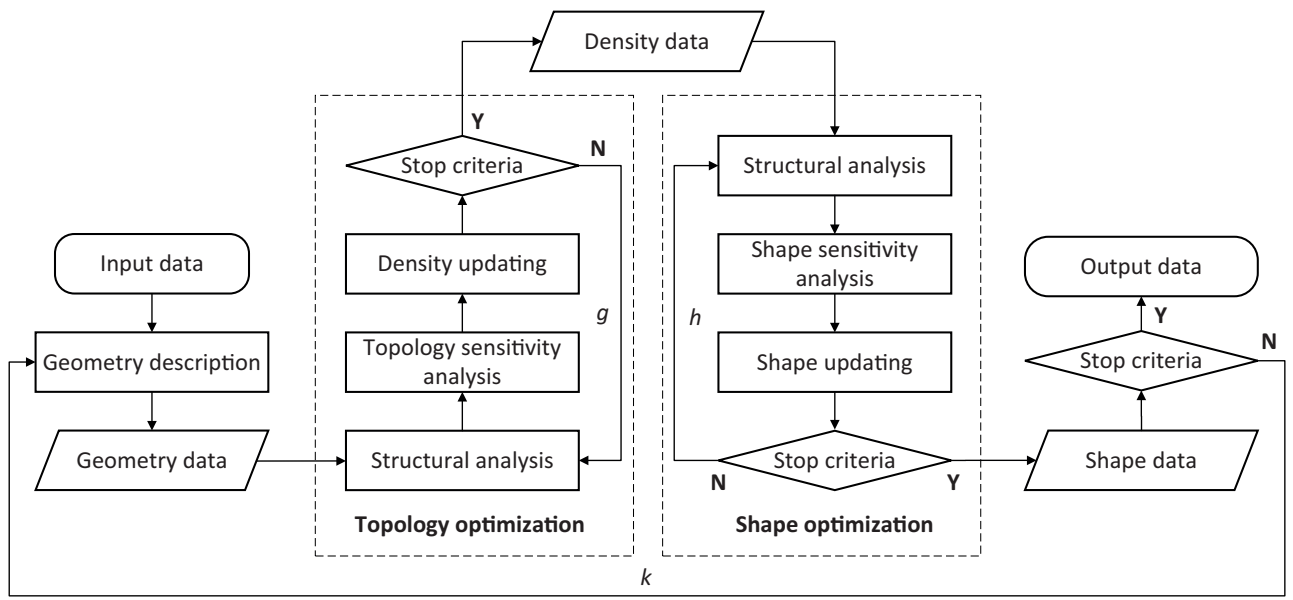

Fig. 5.5. Flowchart of the staggered update scheme for coupled optimization, with iterations $g$ and $h$ referring to topology and shape optimization loops, respectively, and iteration $k$ referring to the outer loop.

In the staggered update scheme, the topology is optimized by momentarily freezing the shape design variables a in Eq. (5.3), which reduces the formulation to

$$
\begin{aligned}
\min _{\boldsymbol{\rho}} & f(\boldsymbol{\rho})=\frac{c(\boldsymbol{\rho})}{c_{0}}, \\
\text { subject to } & \frac{V(\boldsymbol{\rho})}{V_{\max }} \leq 1, \\
\text { with } & 0 \leq \rho_{\min } \leq \rho_{e} \leq 1, \quad e=1, \ldots, E .
\end{aligned}
$$

Similarly, the shape is optimized by keeping the topology design variables $\rho$ in Eq. (5.3) temporarily fixed, leading to 


$$
\begin{aligned}
& \min _{\mathbf{a}} \quad f(\mathbf{a})=\frac{c(\mathbf{a})}{c_{0}}, \\
& \text { subject to } \quad \frac{V(\mathbf{a})}{V_{\max }} \leq 1 \text {, } \\
& \mathbf{A a} \leq \mathbf{b} \\
& \mathbf{h}_{g}(\mathbf{a}) \leq \mathbf{0}, \quad g=1, \ldots, G, \\
& \text { with } \quad 0 \leq \frac{a_{s}-l_{s}}{u_{s}-l_{s}} \leq 1, \quad s=1, \ldots, S .
\end{aligned}
$$

More details on the above staggered update scheme can be found in [119, 131].

\subsubsection{Sensitivity analysis}

In the coupled optimization framework the shape and topology design variables a and $\boldsymbol{\rho}$ are efficiently updated by adopting a gradient-based solution method. For this purpose, the shape and topology sensitivities need to be derived in closed form, as described below.

\subsubsection{Shape sensitivities}

In accordance with Eq. 5.1 and Figure 5.1, the location $\mathbf{p}=[x, y]^{\mathrm{T}}$ of an arbitrary node in the $2 \mathrm{D}$ mesh of a beam cross-section is obtained from the location of this node in the cross-sectional design unit, $\mathbf{p}_{\mathrm{a}}=\left[x_{\mathrm{a}}, y_{\mathrm{a}}\right]^{\mathrm{T}}$, by applying a projection via the geometry function G. Correspondingly, the partial derivative of the location of a node $\mathbf{p}$ with respect to the shape design variable $a_{s}$ is given by

$$
\frac{\partial \mathbf{p}}{\partial a_{s}}=\frac{\partial \mathbf{G}\left(\mathbf{p}_{\mathbf{a}}\right)}{\partial a_{s}}=\frac{\partial \mathbf{G}\left(\mathbf{p}_{\mathbf{a}}\right)}{\partial l} \frac{\partial l}{\partial a_{s}}+\frac{\partial \mathbf{G}\left(\mathbf{p}_{\mathbf{a}}\right)}{\partial \beta} \frac{\partial \beta}{\partial a_{s}},
$$

with

$$
\frac{\partial \mathbf{G}\left(\mathbf{p}_{\mathrm{a}}\right)}{\partial l}=\mathbf{R}\left(\mathbf{p}_{\mathrm{a}}-\mathbf{o}_{\mathrm{c}}\right), \quad \frac{\partial \mathbf{G}\left(\mathbf{p}_{\mathrm{a}}\right)}{\partial \beta}=l \frac{\partial \mathbf{R}}{\partial \beta}\left(\mathbf{p}_{\mathrm{a}}-\mathbf{o}_{\mathrm{c}}\right),
$$

where $\partial \mathbf{R} / \partial \beta$ follows from Eq. 5.2 and $\partial l / \partial a_{s}$ and $\partial \beta / \partial a_{s}$ can be determined in closed form using NURBS theory, see [89, 131] for more details. Using Eqs. (5.4) and (5.5), the partial derivatives of the nodal displacements $\mathbf{u}$ and the structural compliance $c$ with respect to shape design variable $a_{s}$ respectively follow as

$$
\frac{\partial \mathbf{u}}{\partial a_{s}}=\mathbf{K}^{-1}\left(\frac{\partial \mathbf{f}}{\partial a_{s}}-\frac{\partial \mathbf{K}}{\partial a_{s}} \mathbf{u}\right),
$$

and 


$$
\frac{\partial c}{\partial a_{s}}=\frac{\partial \mathbf{f}^{\mathrm{T}}}{\partial a_{s}} \mathbf{u}+\mathbf{f}^{\mathrm{T}} \frac{\partial \mathbf{u}}{\partial a_{s}} .
$$

Inserting Eq. (5.12) into Eq. (5.13) and invoking Eq. (5.5) leads to

$$
\frac{\partial c}{\partial a_{s}}=\frac{\partial \mathbf{f}^{\mathrm{T}}}{\partial a_{s}} \mathbf{u}+\mathbf{u}^{\mathrm{T}} \frac{\partial \mathbf{f}}{\partial a_{s}}-\mathbf{u}^{\mathrm{T}} \frac{\partial \mathbf{K}}{\partial a_{s}} \mathbf{u},
$$

where the nodal displacement vector $\mathbf{u}$ follows from solving Eq. (5.5). Note that the term $\partial \mathbf{f} / \partial a_{s}$ vanishes if the external loads $\mathbf{f}$ are independent of the shape design variable $a_{s}$. Alternatively, $\partial \mathbf{f} / \partial a_{s}$ may be characterized by the relation between the external load and the outer shape of the beam; a specific example is given in [119], which provides the sensitivities of an aerodynamic loading with respect to the shape design variables parametrizing an HAWT rotor blade. These sensitivities will be used for the shape and topology optimization of an HAWT rotor blade, as presented in Section 5.4.2 Furthermore, the term $\partial \mathbf{K} / \partial a_{s}$ appearing in Eq. (5.14) is calculated by following the procedure described in [131, and includes the analytical derivatives of the node locations with respect to the shape design variables, as determined from Eqs. (5.10) and (5.11). Finally, the derivative of the objective function with respect to the shape design variables results from Eq. (5.3) as $\partial f / \partial a_{s}=\left(\partial c / \partial a_{s}\right) / c_{0}$, with the derivative $\partial c / \partial a_{s}$ given by Eq. (5.14).

\subsubsection{Topology sensitivities}

The derivative of the objective function with respect to the topology design variables is obtained from Eq. (5.3) as $\partial f / \partial \rho_{e}=\left(\partial c / \partial \rho_{e}\right) / c_{0}$, whereby $\partial c / \partial \rho_{e}$ may be directly deduced from the form given by Eq. (5.14) and using the fact that the external load vector is independent of the topology design variables, $\partial \mathbf{f} / \partial \rho_{e}=\mathbf{0}$, i.e.,

$$
\frac{\partial c}{\partial \rho_{e}}=-\mathbf{u}^{\mathrm{T}} \frac{\partial \mathbf{K}}{\partial \rho_{e}} \mathbf{u}
$$

Here, the computation of $\partial \mathbf{K} / \partial \rho_{e}$ is performed in accordance with the procedure described in [131]. However, in order to alleviate possible checkerboard patterns in the calculated element densities, Eq. (5.15) is slightly adapted by applying the sensitivity filter proposed in [66, such that

$$
\frac{\partial \hat{c}}{\partial \rho^{e}}=\frac{1}{\rho^{e} \sum_{f=1}^{N} \hat{H}^{f}} \sum_{f=1}^{N} \hat{H}^{f} \rho^{f} \frac{\partial c}{\partial \rho^{f}},
$$

in which

$$
\hat{H}_{f}=\max \left(0, r_{\min }-\operatorname{dist}(e, f)\right),
$$


where $\operatorname{dist}(e, f)$ is the distance between the center of element $e$ and the center of element $f$, and $r_{\min }$ is the radius adopted for the sensitivity filter. In the classical SIMP approach the radius of the filter can be taken as constant, since the mesh of the design domain is kept fixed. However, in the current coupled optimization approach the element size is variable as a result of the shape optimization procedure, whereby a constant filter size might cause the filter to stop working when the element size increases. For this reason, the radius of the filter is here defined as $r_{\text {min }}=r_{0} \sqrt{A_{e}}$, where $A_{e}$ is the area of element $e$, and $r_{0}$ is a scaling parameter for the radius, which is set to 1.5 for the numerical examples presented in Section 5.4. An alternative version of the sensitivity filter that takes varying element sizes into account has been presented in 94 .

\subsubsection{Update of design variables}

The numerical update procedure of the design variables in the monolithic and staggered schemes is performed using the Sequential Quadratic Programming (SQP) method 95. with the fmincon solver in MATLAB (version R2018a). The first-order sensitivities required for the fmincon solver follow from the expressions presented in Section 5.3.3, and the second-order sensitivities (Hessian matrix) are approximated using the Broyden-Fletcher-Goldfarb-Shanno (BFGS) scheme [95].

\section{$5.4 \quad$ Numerical examples}

The main features of the coupled shape and topology optimization method are illustrated by first analyzing thin-walled beam configurations with a regular cross-section, i.e., a circular and a square tube clamped at one side and loaded by a point load and/or torsional moment at the other side. For simplicity, the external load $\mathbf{f}$ imposed on the beam is hereby assumed to be independent of the shape design variables $\mathbf{a}$. Subsequently, the coupled optimization approach is applied for an HAWT rotor blade, whereby the shape-dependent aerodynamic loading is accounted for together with a constraint condition for the rotor power coefficient in order to warrant an adequate aerodynamic performance. The above optimization problems are solved with the three numerical update schemes presented in Section 5.3.2, which are the monolithic scheme referred to as Concurrent Optimization (CO), and the two staggered schemes referred to as Coupled Shape and Topology Optimization (CSTO) and Coupled Topology and Shape Optimization (CTSO). Accordingly, the numerical efficiency of each update scheme and the uniqueness of the computational result can be demonstrated.

\subsubsection{Circular and square thin-walled cantilever beams}

Figure 5.6(a) illustrates a thin-walled cantilever beam of length $L_{0}$ subjected at its right end to three different loading cases, namely: Case 1, a vertical force $F_{y}$, Case 2 , a torsional moment $M_{z}$, and Case 3, a combination of $F_{y}$ and $M_{z}$. The parameters 
used in the optimization procedure are listed in Table 5.1. Note that the wall thickness $t_{0}$ in Figures 5.6(b) and (c) and Table 5.1 is dimensionless, since the cross-sectional design units in Figures 5.6(b) and (c) are normalized by the scaling factor $l$, see also Section 5.2. The computational results presented are normalized such that they become independent of the Young's modulus $E$ of the material. The Poisson's ratio of the material is set equal to $\nu=0.3$.

(a) Geometry and loading conditions

(b) Circular design unit

(c) Square design unit

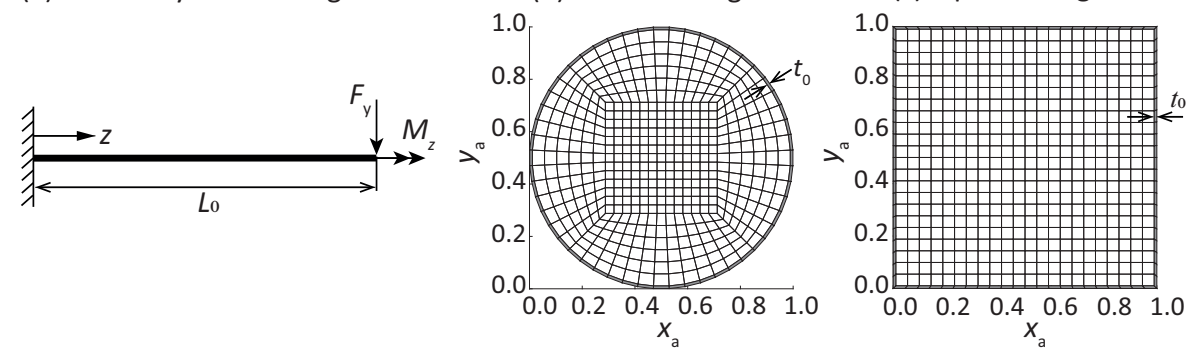

Fig. 5.6. A thin-walled cantilever beam subjected to a vertical point load $F_{y}$ and/or a torsional moment $M_{z}$. (a) Geometry and loading conditions, (b) a circular design unit, and (c) a square design unit.

Table 5.1: Parameter values for the thin-walled cantilever beam problem.

\begin{tabular}{lllll}
\hline Parameter & $L_{0}(\mathrm{~m})$ & $l_{0}(\mathrm{~m})$ & $t_{0}$ & $V_{\max }\left(\mathrm{m}^{3}\right)$ \\
\hline Value & 30 & 1 & 0.01 & 9 \\
\hline Parameter & $F_{y}(\mathrm{kN})$ & $M_{z}(\mathrm{kN} \cdot \mathrm{m})$ & $E(\mathrm{MPa})$ & $\nu$ \\
\hline Value & 10 & 10 & $210 \times 10^{3}$ & 0.3 \\
\hline
\end{tabular}

Two different cross-sectional design units are considered, representative of a circular tube, see Figure 5.6(b), and a square tube, see Figure 5.6(c), which are characterized by an initial diameter and width $l_{0}$, respectively, and an initial dimensionless wall thickness $t_{0}$. The circular and square design units are meshed by 4-node quadrilateral finite elements, with the FEM discretizations also shown in Figure 5.6. In the longitudinal direction of the beam the cross-sectional stiffness properties are computed from 11 cross-sections located at equal mutual distance. Only the first cross-section has a minor offset of $0.01 \mathrm{~m}$ from the beam's left end, by which its properties become representative of the beam part starting at the left beam end up to this location. The cross-sectional design units shown in Figures 5.6(b) and (c) are mapped to the corresponding cross-sections using the geometrical projection in Eq. (5.1). The design unit's reference point $\mathbf{o}_{\mathrm{c}}$ appearing in Eq. (5.1) equals $\mathbf{o}_{\mathrm{c}}=[0.5,0.5]^{\mathrm{T}}$ for both the circular and square design units, see Figures 5.6 (b) and (c), and the cross-sectional reference point $\mathbf{o}_{\mathrm{b}}=[0,0]^{\mathrm{T}}$ is considered to coincide with the central axis of the beam.

For simplicity, in the present analyses the cross-sectional design units will be scaled but not twisted, i.e., in Eq. (5.1) only the scaling factor $l$ is activated and the twist 
angle $\beta$ is set to zero. However, the contribution by the twist angle will be included in the shape optimization of an HAWT rotor blade presented in Section 5.4.2. For the circular design unit the scaling factor $l$ scales its initial diameter, while for the square design unit it scales its initial width. The axial distribution of the scaling factor $l$ is defined by 5 control points characterizing the NURBS curve, see Figure 5.2 (a). The control points are free to move horizontally, except for the endpoints, which are kept fixed in order to keep the beam length constant. Accordingly, the shape design variables are defined by

$$
\boldsymbol{a}=\left[L_{1}, Z_{2}, L_{2}, Z_{3}, L_{3}, Z_{4}, L_{4}, L_{5}\right]^{\mathrm{T}}
$$

where $L_{s}$ (with $s=1, \ldots, 5$ ) is the scaling factor of the 5 control points and $Z_{s}$ represents their normalized $z$-coordinate in the longitudinal beam direction, see Figure 5.6 . Initially the scaling factor is prescribed to be uniform along the beam length, $l=l_{0}$, with the value of $l_{0}$ presented in Table 5.1. Correspondingly, the initial values of $\boldsymbol{a}$ are defined as $[1,0.2,1,0.5,1,0.9,1,1]^{\mathrm{T}}$. Further, their lower and upper bounds are set to $[0.3,0.1,0.3,0.4,0.3,0.8,0.3,0.3]^{\mathrm{T}}$ and $[1.5,0.3,1.5,0.7,1.5,0.95,1.5,1.5]^{\mathrm{T}}$, respectively. The initial value $\rho_{0}$ of the topology design variables is determined by Eq. (5.7), which, with the values of $V_{\max }$ and $t_{0}$ presented in Table 5.1, leads to $\rho_{0}=0.358$ and $\rho_{0}=0.271$ for the circular and square design units, respectively. The coupled optimization process is assumed to be converged when the objective function in Eq. (5.3) satisfies the stop criterion $\left|f^{k+1}-f^{k}\right| \leq 1 e-5$, with $k$ the iteration number. For the staggered CTSO and CSTO schemes, the stop criteria of the inner loops are set as $\left|f^{g+1}-f^{g}\right| \leq 1 e-5$ and $\left|f^{h+1}-f^{h}\right| \leq 1 e-5$, with $g$ and $h$ the iteration numbers for topology and shape optimization, respectively.

The optimization results will be first discussed for the cantilever beam with a circular thin-walled cross-section. For loading Case 1, whereby the right end of the beam is subjected to a vertical force $F_{y}$, the optimization results obtained by the monolithic CO scheme and the staggered CTSO and CSTO schemes are shown in Figures 5.7, 5.8 and 5.9 , respectively. Here, the normalized structural compliance $c / c_{0}$ is plotted versus the total number of iterations $k$, with the inset illustrating how the cross-section at the left beam end has evolved after various numbers of iteration $k$. The final cross-section computed by the three update schemes turns out to be comparable; it is represented by a cylindrical shell that is internally reinforced by spar caps at the top and bottom of the cross-section, which clearly maximizes the bending resistance of the beam. A similar cross-sectional shape is found halfway the beam length, as illustrated in Figures 5.10(a), (b) and (c) for the three update schemes. Note that for all three numerical update schemes the final, optimized configuration hardly contains intermediate densities; the configurations are essentially composed by parts with a density equal to unity (coloured red) and zero (coloured dark blue). Here, the upper and lower cross-sectional parts with a density equal to unity are connected to each other via the outer thin wall of which the density during the optimization is kept fixed at unity, so that the optimized configuration is physically admissible. The overall shape of the beam structure is tapered, which can be concluded from the final distribution 
of the scaling factor $l$ in the longitudinal direction of the beam, see Figure 5.11(a). Obviously, this shape results from the fact that the bending moment generated by the point load $F_{y}$ increases linearly from zero at the loading point to a maximum value of $F_{y} L_{0}$ at the clamped left end. Note from Figure 5.10 that the outer beam diameters following from the $\mathrm{CO}$ and CSTO update schemes are comparable, whereas the beam diameter calculated with the CTSO scheme is substantially smaller. Correspondingly, the final value of the normalized structural compliance is the lowest for the CSTO approach, $c / c_{0}=0.075$, closely followed by the $\mathrm{CO}$ approach, $c / c_{0}=0.078$, and finally by the CTSO approach, $c / c_{0}=0.109$, with the compliance value of the CTSO approach being $(0.109-0.075) / 0.109 \times 100 \%=31 \%$ larger than that of the CSTO approach.

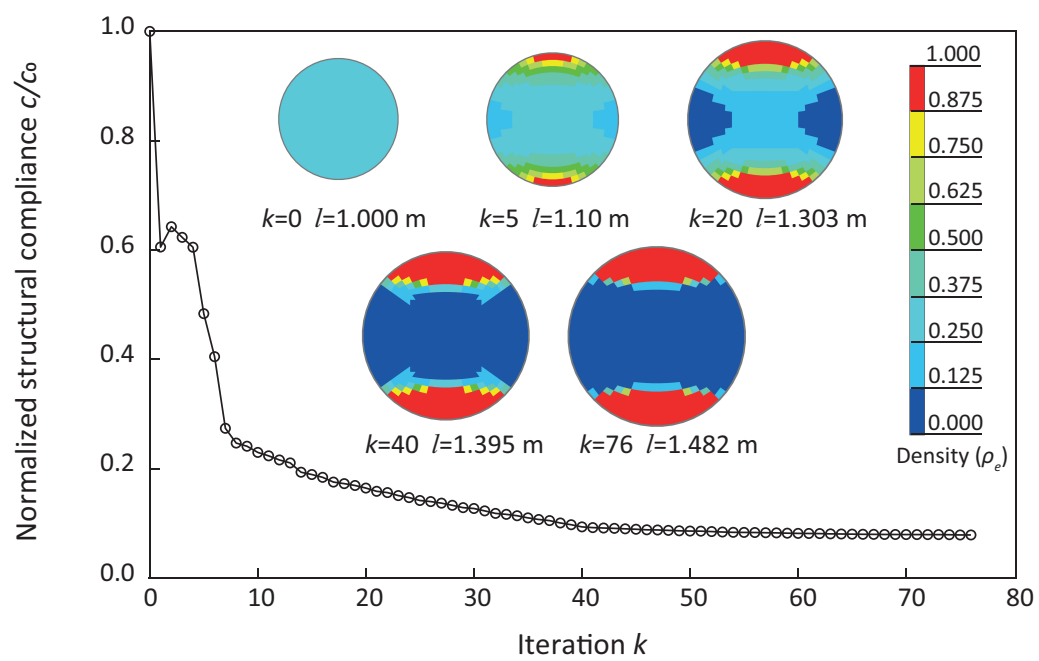

Fig. 5.7. Concurrent optimization (CO): convergence behavior of the normalized compliance $c / c_{0}$ for a cantilever beam with a circular design unit subjected to a vertical for $F_{y}$ (loading Case 1). The inset shows the evolution of the first cross-section (at the left side of the beam) after various numbers of iterations $k$.

It can be observed from Figures 5.7, 5.8 and 5.9 that the number of iterations $k$ required for reaching a converged solution is the lowest for the monolithic CO approach, $k=76$, followed by the staggered CSTO approach, $k=108$, and finally the staggered CTSO approach, $k=127$. Since the shape and topology design variables in the monolithic and two staggered schemes are updated in a different fashion, see the flowcharts in Figures 5.4 and 5.5, the number of iterations $k$ cannot be directly related to the computational efficiency of the update schemes. This can be indeed confirmed from the values listed in Table 5.2 , which show that the order in computational times is different from the order in the number of iterations $k$ mentioned above: for loading Case 1 the CO approach relates to the shortest computational time, followed by the CTSO and the CSTO approaches, where the maximal relative difference in computational time is equal to a factor 1.629. Additionally, the other two loading cases, Cases 


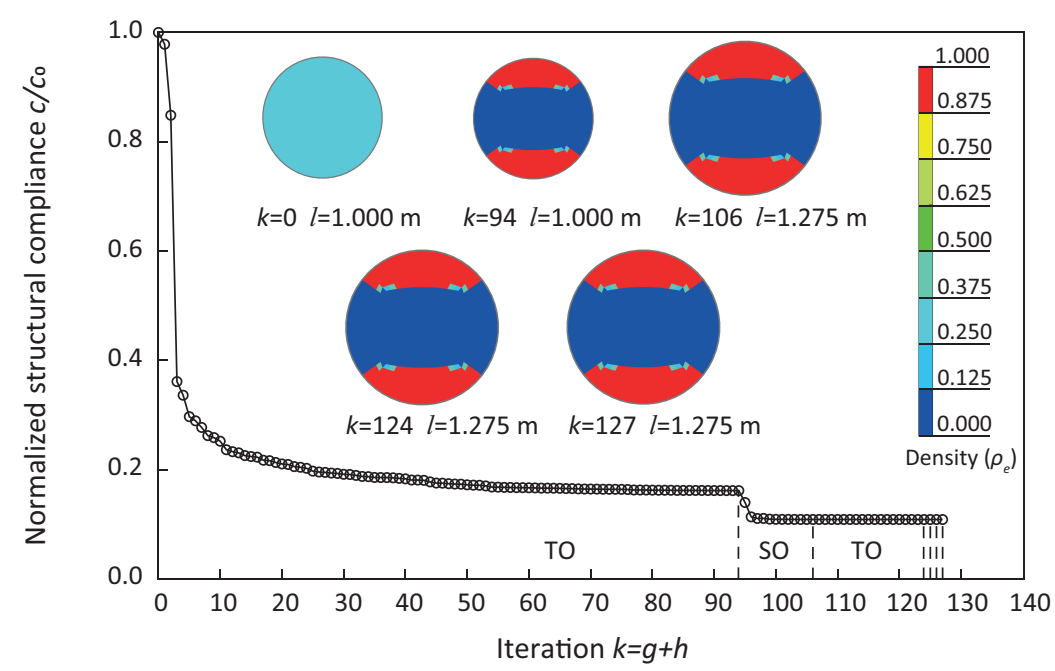

Fig. 5.8. Coupled Topology and Shape Optimization (CTSO): convergence behavior of the normalized compliance $c / c_{0}$ for a cantilever beam with a circular design unit subjected to a vertical for $F_{y}$ (loading Case 1). The inset shows the evolution of the first cross-section (at the left side of the beam) after various numbers of iterations $k$.

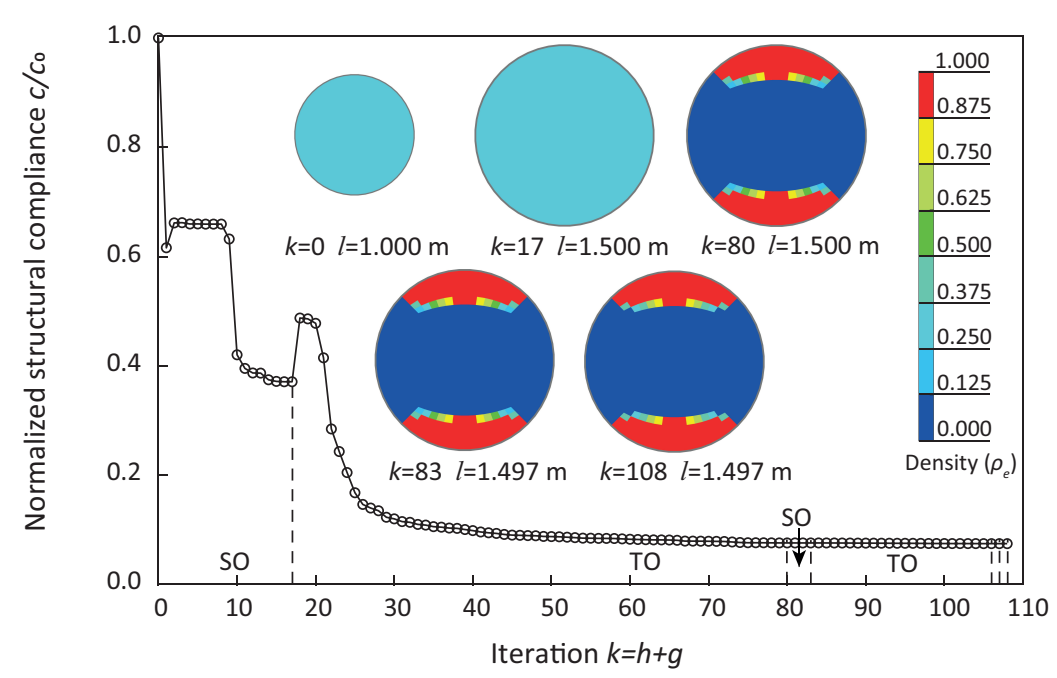

Fig. 5.9. Coupled Shape and Topology Optimization (CSTO): convergence behavior of the normalized compliance $c / c_{0}$ for a cantilever beam with a circular design unit subjected to a vertical for $F_{y}$ (loading Case 1). The inset shows the evolution of the first cross-section (at the left side of the beam) after various numbers of iterations $k$. 
2 and 3, show a different order in computational time, which demonstrates the dependency of the computational efficiency of the update scheme on the characteristics of the boundary value problem.

Table 5.2: Relative computational time for cantilever beam with a circular design unit.

\begin{tabular}{llll}
\hline & Case 1 & Case 2 & Case 3 \\
\hline CTSO/CO & 1.054 & 0.617 & 1.133 \\
\hline CSTO/CO & 1.629 & 0.678 & 2.223 \\
\hline CTSO/CSTO & 0.647 & 0.910 & 0.509 \\
\hline
\end{tabular}

Table 5.3: Relative computational time for cantilever beam with a square design unit.

\begin{tabular}{llll}
\hline & Case 1 & Case 2 & Case 3 \\
\hline CTSO/CO & 0.651 & 0.819 & 1.300 \\
\hline CSTO/CO & 0.852 & 0.777 & 3.631 \\
\hline CTSO/CSTO & 0.764 & 1.054 & 0.358 \\
\hline
\end{tabular}

Consider now the optimization results for the circular thin-walled cantilever beam under loading Case 2, whereby the right beam end is subjected to a torsional moment $M_{z}$. From Figure 5.11(c), it can be seen that for the CTSO and CSTO update schemes the cross-section maintains its initial diameter of $l=l_{0}=1.0 \mathrm{~m}$ along the complete beam length, indicating that shape optimization (virtually) has no influence on the optimization process. Conversely, for the CO update scheme the cross-sectional diameter reduces in value to $l=0.869 \mathrm{~m}$, whereby the final wall thickness of the tube is larger than for the CTSO and CSTO approaches, see Figures 5.10(d), (e) and (f). Since the external torsional moment generates a constant torsional moment distribution, the shape of the beam remains constant in the longitudinal direction. The final normalized compliance $c / c_{0}$ computed by the $\mathrm{CO}$ approach turns out to be $(0.293-0.210) / 0.210 \times 100 \%=40 \%$ larger than for the CTSO and CSTO approaches, and thus is less optimal. When comparing the order of the compliance values for loading Case 2 with that of loading Case 1, see Figure 5.10, it may be concluded that it depends on the type of loading condition which of the three update schemes provides the lowest compliance.

For loading Case 3, which is a combination of the loading Cases 1 and 2, the optimization results for the circular, thin-walled cantilever beam appear to be relatively close to those obtained for loading Case 1, as can be concluded from the comparison of Figures 5.11(a) and 5.11(e), and of Figures 5.10(a), (b), (c) and 5.10(g), (h), (i). Hence, it may be concluded that the optimization of the shape and topology of the thin-walled beam under loading Case 3 is mainly determined by the external point load $F_{y}$. Obviously, when increasing the magnitude of $M_{z}$ presented in Table 5.1, the contribution of the torsional moment to the optimization result of Case 3 will become more prominent. 


\subsection{Numerical examples}

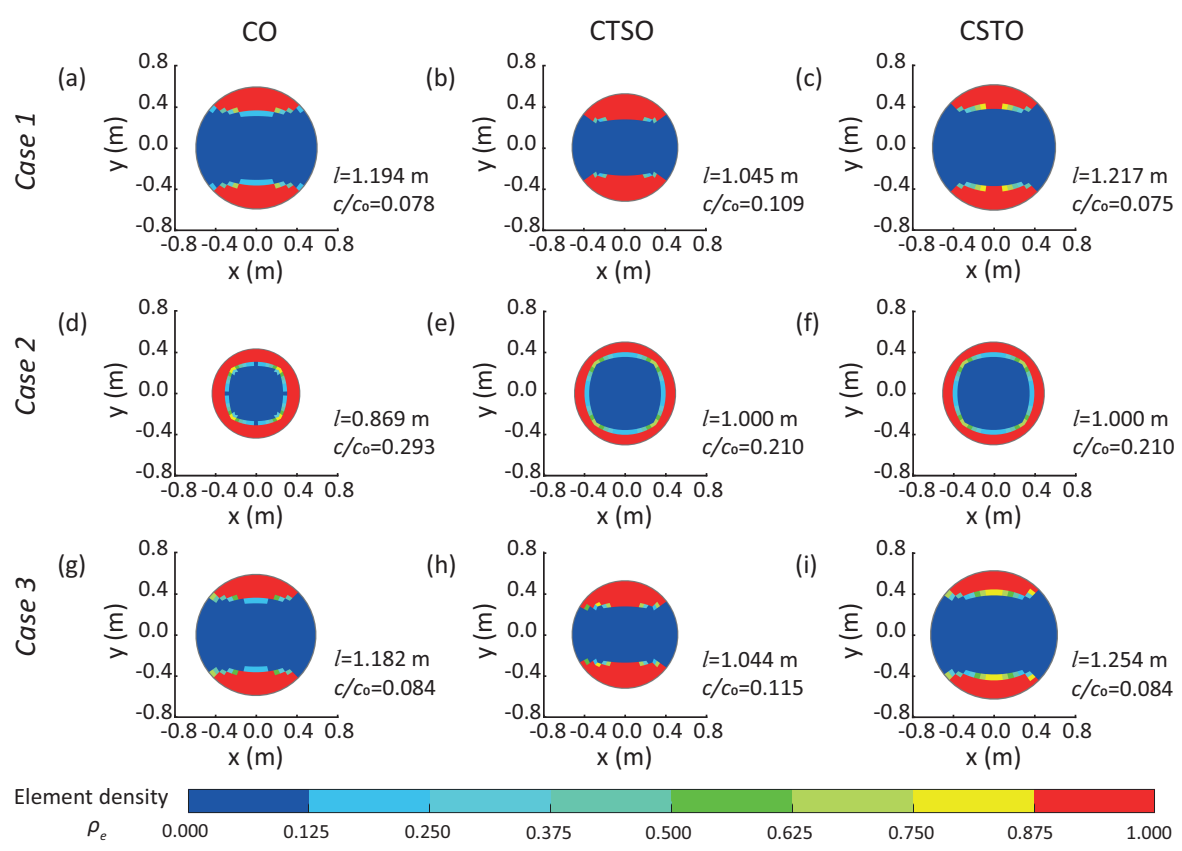

Fig. 5.10. Final configuration of the 6 th cross-section of the cantilever beam (located at the half-length) with the circular design unit. Loading case 1 (vertical force $F_{y}$ ) with the $\mathrm{CO}$ (a), CTSO (b) and CSTO (c) numerical update schemes. Loading case 2 (torsional moment $M_{z}$ ) with the CO (d), CTSO (e) and CSTO (f) numerical update schemes. Loading case 3 (vertical force $F_{y}$ and torsional moment $M_{z}$ ) with the CO (g), CTSO (h) and CSTO (i) numerical update schemes.

For the cantilever beam with a square, thin-walled cross-section, the optimization results are qualitatively comparable to those of the thin-walled beam with a circular cross-section, both in terms of the variation of the shape in longitudinal direction, see Figure 5.11, and the distribution of material across the cross-section, see Figures 5.10 and 5.12 . The latter two figures further show that for loading Cases 1 and 2 the numerical update scheme resulting in the lowest relative compliance $c / c_{0}$ is different for the circular and square cross-sections, which indicates that the performance of the update scheme depends on the actual geometry considered. In fact, this conclusion not only applies to the specific value computed for $c / c_{0}$, but also to the computational efficiency of the update scheme, see Tables 5.2 (circular cross-section) and 5.3 (square cross-section).

In summary, the three different update schemes result in comparable design concepts, but lead to different values for the minimal structural compliance. Since the monolithic CO scheme typically navigates a larger design space than the staggered CTSO and CSTO schemes, for most cases examined it provides the lowest structural compliance. An exception to this rule follows from Case 2 of a cantilever beam with a circular design unit subjected to a torsional moment $M_{z}$, whereby the CO scheme gets 

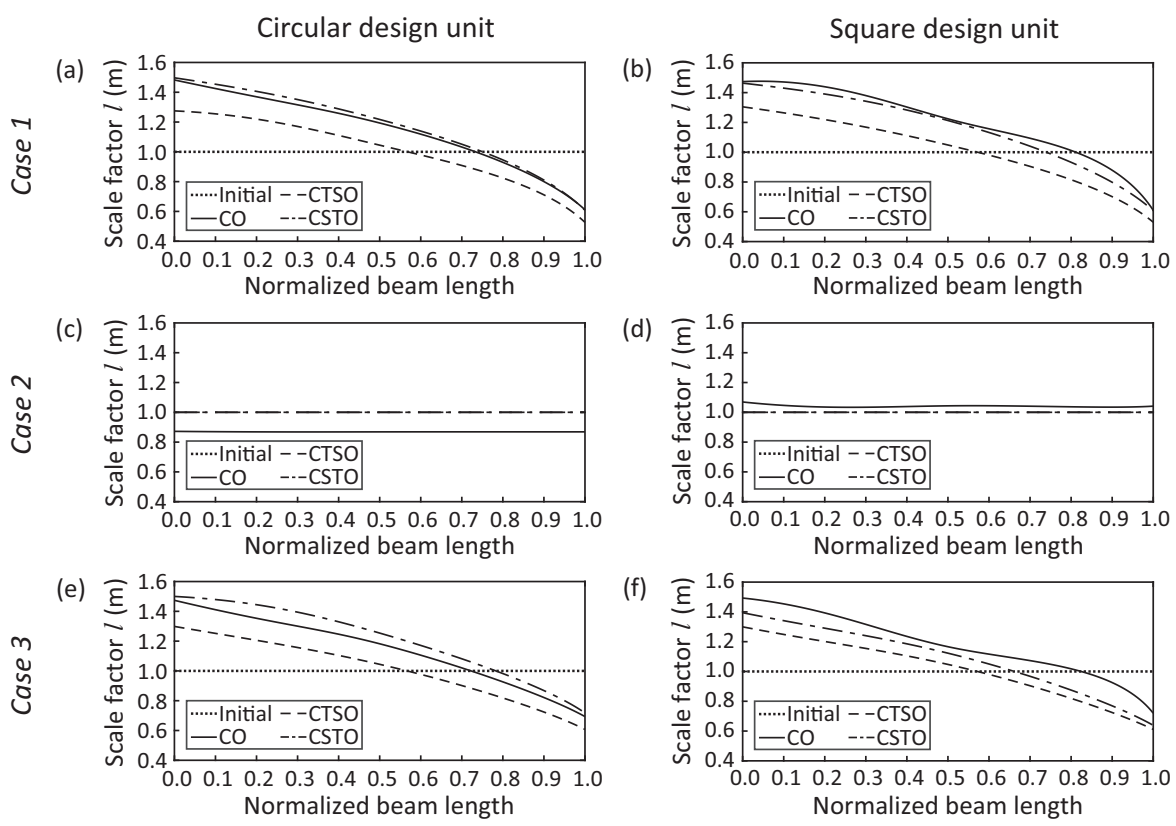

Fig. 5.11. Final distribution of the scale factor $l$ in the longitudinal direction of the cantilever beam for the CO, CTSO and CSTO update schemes. Loading case 1 (vertical force $F_{y}$ ) with circular (a) and square (b) design units. Loading case 2 (torsional moment $M_{z}$ ) with circular (c) and square (d) design units. Loading case 3 (vertical force $F_{y}$ and torsional moment $M_{z}$ ) with circular (e) and square (f) design units.

stuck in a less optimal, local minimum that ignores the fact that under uniform torsion shape optimization (virtually) has no influence on the final, optimized beam configuration. Furthermore, for the two staggered update schemes, the CSTO scheme starting with a shape optimization step commonly enlarges the design space for the subsequent topology optimization step, such that the final compliance calculated by this scheme may be lower than the value computed by the CTSO scheme, and thereby closer to the compliance value following from the monolithic $\mathrm{CO}$ scheme. In the section below the aspects outlined above are further investigated by considering a practical case study related to a rotor blade of a horizontal-axis wind turbine (HAWT). In the formulation of the structural optimization problem, the shape-dependent aerodynamic loading is accounted for together with a constraint condition for the rotor power coefficient in order to warrant an adequate aerodynamic performance.

\subsubsection{HAWT rotor blade}

Consider a HAWT for which the rotor blades are characterized by the airfoil crosssectional design unit depicted in Figure 5.1(a). This standard airfoil type is commonly referred to as "DU 93-W-210", with the specific design details presented in [162]. The 


\subsection{Numerical examples}

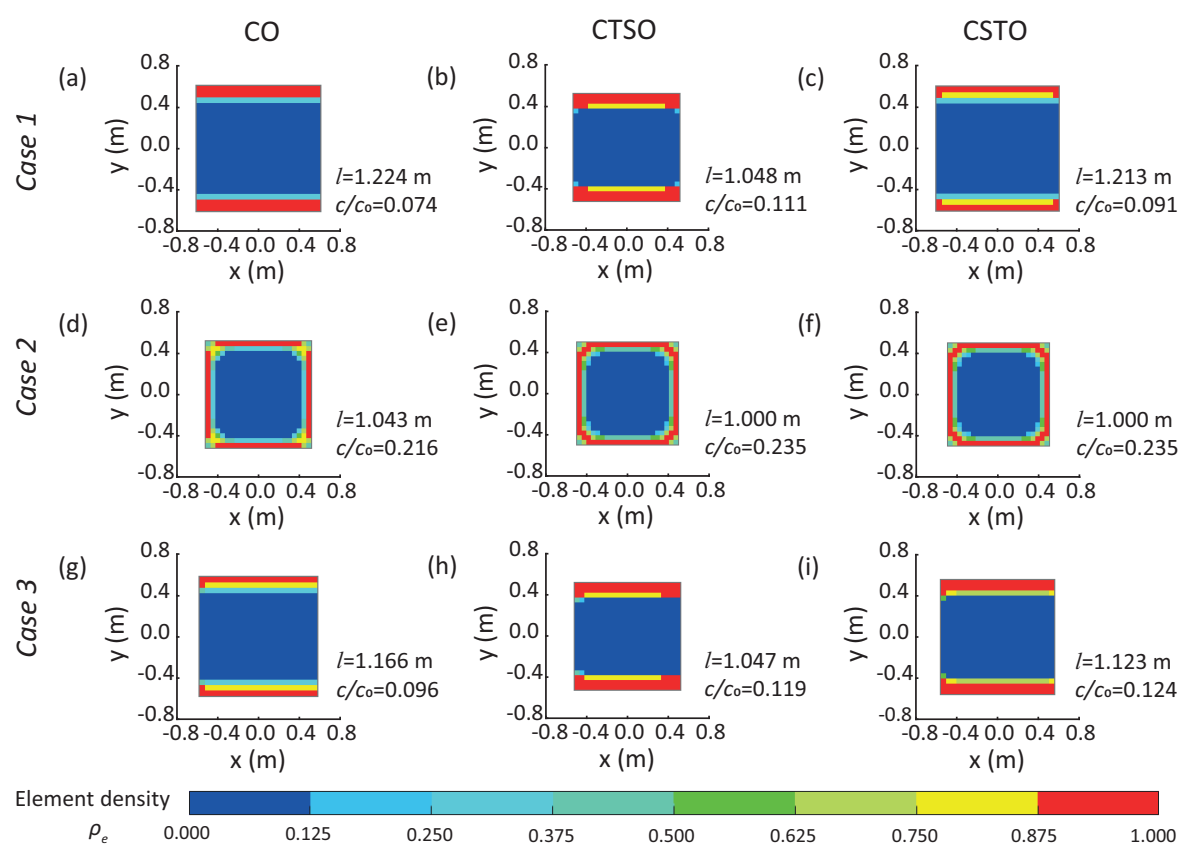

Fig. 5.12. Final configuration of the 6th cross-section of the cantilever beam (located at the half-length) with the square design unit. Loading case 1 (vertical force $F_{y}$ ) with the $\mathrm{CO}$ (a), CTSO (b) and CSTO (c) numerical update schemes. Loading case 2 (torsional moment $M_{z}$ ) with the CO (d), CTSO (e) and CSTO (f) numerical update schemes. Loading case 3 (vertical force $F_{y}$ and torsional moment $M_{z}$ ) with the CO (g), CTSO (h) and CSTO (i) numerical update schemes.

11 airfoil cross-sections modeling the total blade geometry are meshed by 6 -node triangular 2D elements, see Figure 5.1(a). The outer wall defining the geometry of the airfoil design unit has a dimensionless thickness of $t_{0}=0.005$, and the other geometrical characteristics are the same as for the thin-walled cantilever beam problems studied in Section 5.4.1, i.e., the blade length equals $L_{0}=30 \mathrm{~m}$ and the initial chord length of the airfoil cross-section is $l_{0}=1 \mathrm{~m}$, see Table 5.1. From the wall thickness mentioned above, the initial volumes of the wall and interior of the blade become $V_{0}^{\mathrm{w}}=0.329 \mathrm{~m}^{3}$ and $V_{0}^{\mathrm{i}}=3.486 \mathrm{~m}^{3}$, respectively. By prescribing the maximum material volume of the blade as $V_{\max }=1.5 \mathrm{~m}^{3}$, the initial value of the topology design variables is obtained via Eq. 5.7 as $\rho_{0}=0.336$. Further, the stop criteria adopted for the update schemes are the same as for the thin-walled cantilever beam problems analyzed in Section 5.4.1

The aerodynamic loads acting on the rotor blade are calculated by applying the Blade Element Momentum (BEM) method [136; the main details of this method can be found in [119]. The aerodynamic lift and drag coefficients of the airfoil are used as input for the BEM method, which, for the standard DU 93-W-210 airfoil, are reported in [133. An example is given in Figure 5.13, showing the lift $c_{L}$ and $\operatorname{drag} c_{D}$ coefficients as a function of the angle of attack $\alpha$ for a Reynolds number 
$R e=1 \times 10^{6}$. By setting the free-stream wind speed as $U_{\infty}=10 \mathrm{~m} / \mathrm{s}$ and the blade tip-speed ratio as $\lambda=9$, from the initial shape of the blade the initial aerodynamic normal and tangential forces on each of the 11 blade cross-sections in the 2.5D FEM model can be calculated, see Figure 5.14 for the result. These aerodynamic forces need to be converted to the load vector $\mathbf{f}$ used in the FEM formulation, Eq. (5.5), which is done by transferring the forces from the aerodynamic center of the blade to the blade reference axis. Since the aerodynamic loads $\mathbf{f}$ are characterized by the outer shape of the blade, the partial derivatives $\partial \mathbf{f} / \partial a_{s}$ of these loads with respect to the shape design variables are calculated in agreement with Eq. (5.14), the details of which are presented in [119.

The shape of the airfoil is optimized by scaling the chord length in accordance with the scaling factor $l$, and twisting the airfoil cross-section by an angle $\beta$. The chord and twist distributions in the longitudinal beam direction are determined by the values of the NURBS control points that act as shape design variables, as represented by $\boldsymbol{a}=\left[\boldsymbol{a}_{\mathrm{s}}^{\mathrm{T}}, \boldsymbol{a}_{\mathrm{t}}^{\mathrm{T}}\right]^{\mathrm{T}}$. Here, $\boldsymbol{a}_{\mathrm{s}}$ reflects the chord distribution and $\boldsymbol{a}_{\mathrm{t}}$ refers to the twist distribution, in correspondence with the mathematical format given by Eq. (5.18). Similar to the examples treated in Section 5.4.1, the initial values of $\boldsymbol{a}_{\mathrm{s}}$ correspond to a uniform chord length $l_{0}=1 \mathrm{~m}$ along the longitudinal beam direction, in accordance with $[1,0.2,1,0.5,1,0.9,1,1]^{\mathrm{T}}$. The lower and upper bounds of $\boldsymbol{a}_{\mathrm{s}}$ are set equal to $[0.3,0.1,0.3,0.4,0.3,0.8,0.3,0.3]^{\mathrm{T}}$ and $[1.5,0.3,1.5,0.7,1.5,0.95,1.5,1.5]^{\mathrm{T}}$, respectively. Additionally, the initial values of $\boldsymbol{a}_{\mathrm{t}}$ are $[0,0.2,0,0.5,0,0.9,0,0]^{\mathrm{T}}$, thus assuming that the blade is initially untwisted, with the lower bound as $[0,0.1,0,0.4,0,0.8,0,0]^{\mathrm{T}}$ and the upper bound as $[10,0.3,10,0.7,10,0.95,10,10]^{\mathrm{T}}$.

During the optimization procedure, the locations of the first control point at the blade root and the fifth control point at the blade tip are kept fixed, so that the blade length remains constant, see Figure 5.2. The locations of the other three control points are allowed to change during the optimization process. In order to meet the so-called "monotonicity requirement" for turbine blade cross-sections [37, the values of both $l$ and $\beta$ in the second, third and fourth control points are enforced to successively decrease. This inequality constraint is implemented in the optimization framework via the matrix $\mathbf{A}$ and vector $\mathbf{b}$ in Eq. (5.3).

The objective function $c$ in Eq. (5.3) aims at minimizing the structural compliance, which naturally drives the optimization result towards a turbine blade shape that minimizes the aerodynamic loading, and thus the power output. In order to obtain a blade design with a sufficiently high power output, the normalized rotor power coefficient $C_{p} / C_{\mathrm{p} 0}$, with $C_{\mathrm{p} 0}$ being the initial value of the rotor power coefficient, has been computed using the program CCBlade [132] and is prescribed to be equal to or larger than 1. This condition is implemented in Eq. (5.3) by means of a nonlinear inequality constraint $\mathbf{h}_{g}$, whereby the sensitivity of the power coefficient with respect to the shape design variables, $\partial C_{\mathrm{p}} / \partial a_{s}$, is calculated in accordance with the procedure presented in [119. Since the main focus of the present study is on the numerical performance of the monolithic and staggered update schemes applied in the optimization procedure, the contribution of the power output to the optimized blade design is taken into account in a relatively simple fashion. A more rigorous optimization approach, which 


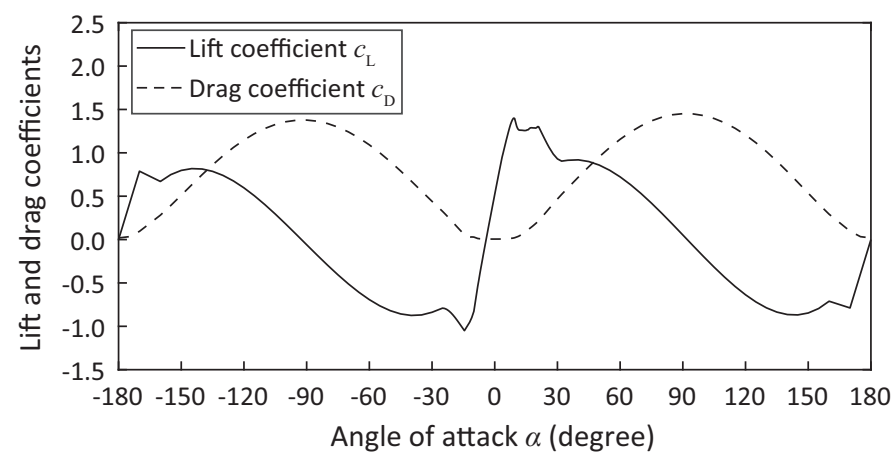

Fig. 5.13. Lift and drag coefficients for the DU $93-\mathrm{W}-210$ airfoil as a function of angle of attack, using a Reynold's number $R e=1 \times 10^{6}$.

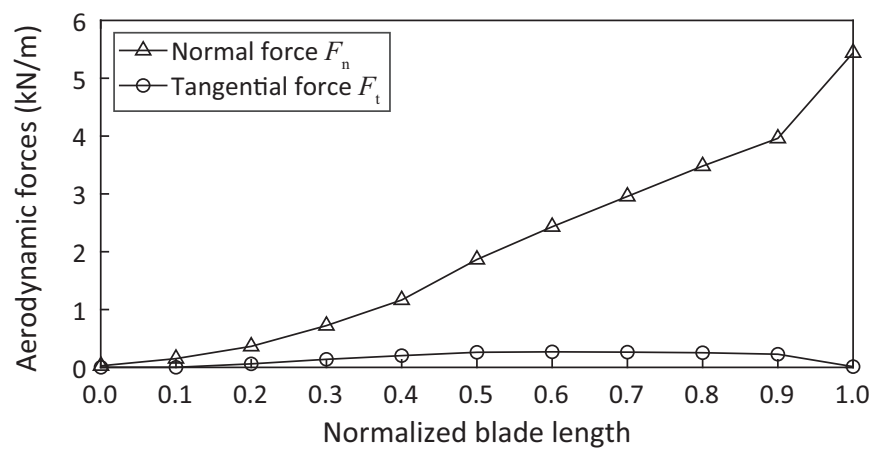

Fig. 5.14. Distributions of the initial normal and tangential aerodynamic forces along the blade length.

minimizes the structural compliance and simultaneously maximizes the power output, requires the present framework to be extended towards a multi-objective optimization formulation, the details of which can be found in [119.

Figure 5.15 illustrates the convergence behavior of the normalized compliance $c / c_{0}$ of the wind turbine blade for the monolithic $\mathrm{CO}$ update scheme, with the inset showing the evolution of the 6th cross-section located at the half-length of the blade. It can be observed that after the first iteration $k=1$ the compliance $c / c_{0}$ has already decreased by approximately $90 \%$ of its initial value, providing the turbine blade with a tapered shape and a material distribution that is still more or less uniform, see Figure 5.16(a). With an increasing number of iterations the compliance decreases further under a simultaneous optimization of the outer shape and the interior topology, see Figure 5.15. It can be observed that at iteration $k=60$ a shear web appears, which subsequently vanishes under a further optimization of the blade structure. The final blade configuration is characterized by the appearance of spar caps at the upper and lower blade surfaces, see Figure 5.16(b). The corresponding distributions of the blade 
chord length $l$ and twist angle $\beta$ depicted in Figures 5.17(a) and (b) confirm the tapered shape of the blade, and show that the twist angle gradually decreases from a maximal value of $10^{\circ}$ at the blade root to a value of $6^{\circ}$ at the blade tip. The distribution of the twist angle of the blade ensures that the angle of attack is sufficiently high for satisfying the prescribed aerodynamic condition on the minimum value of the rotor power coefficient.

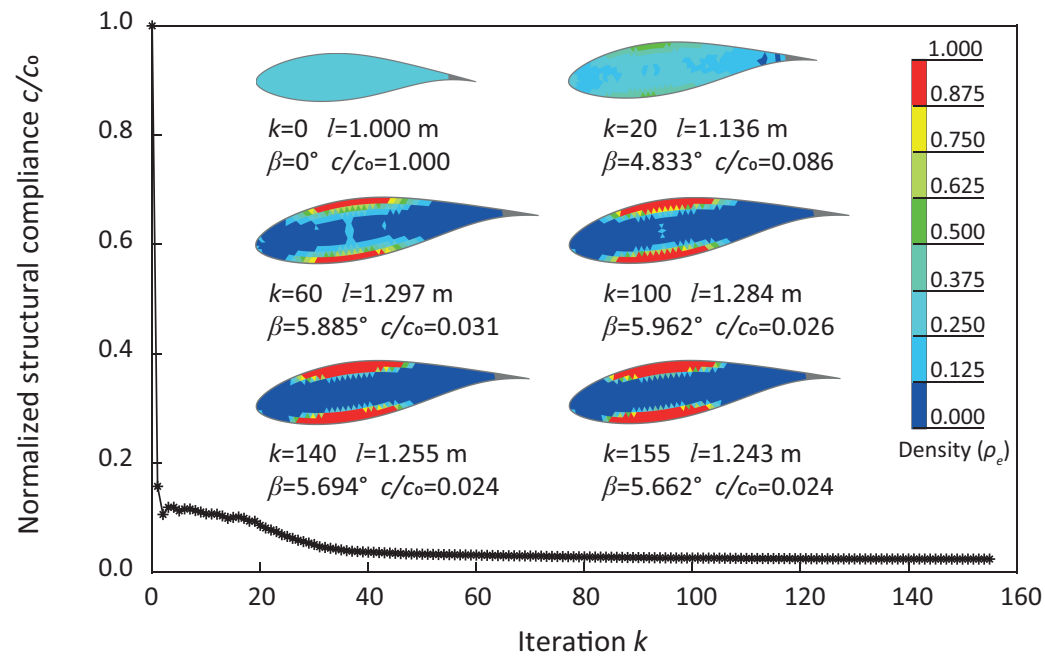

Fig. 5.15. Concurrent optimization $(\mathrm{CO})$ : convergence behavior of the normalized compliance $c / c_{0}$ of the HAWT rotor blade. The inset shows the evolution of the 6th cross-section (located at the half-length of the rotor blade) after various numbers of iterations $k$.

Figures 5.18 and 5.19 present the convergence behavior of the structural compliance $c / c_{0}$ and the corresponding evolution of cross-sectional configurations for the staggered CTSO and CSTO update schemes, respectively. After the first iteration $k=1$ the CSTO approach with a shape optimization in the first step clearly leads to substantially larger reduction of the compliance $c / c_{0}$ than the CTSO approach with a topology optimization in the first step. This demonstrates the large efficiency of shape optimization in lowering the compliance during the coupled optimization process. In addition, the subsequent differences in the convergence behavior of the two approaches indicate that their solution paths in the design space are rather different. Nevertheless, they surprisingly lead to the same final value of the minimal compliance, $c / c_{0}=0.034$, and to a similar internal topology of two spar caps connected by a shear web, see Figures 5.20 (a) and (b). Also, the chord and twist distributions of the CSTO and CTSO approaches are virtually the same, but clearly differ from the distributions found for the monolithic CO approach, see Figure 5.17. This result is consistent with the fact that the final value of the compliance $c / c_{0}$ for the monolithic CO approach is $(1-0.024 / 0.034) \times 100 \%=29 \%$ lower than that of the staggered CSTO and CTSO approaches. Further, the total computational time of the CO approach is $53 \%$ and $12 \%$ less than the computational times of the CTSO and CSTO approaches, from 
(a) First iteration $(k=1)$

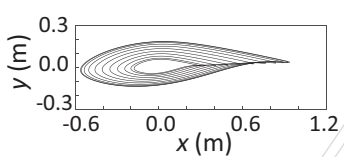

(2) Front view of crosssectional profiles

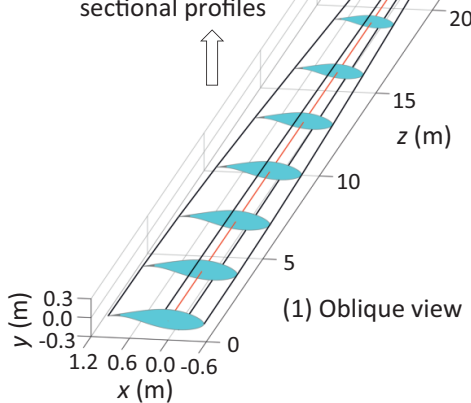

(b) Last iteration $(k=155)$

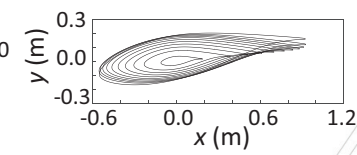

(2) Front view of crosssectional profiles

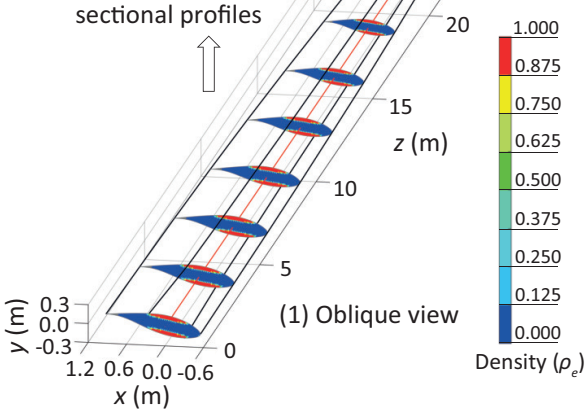

Fig. 5.16. Concurrent optimization $(\mathrm{CO})$ : blade configuration (outer shape and interior material distribution) after the first $(k=1)$ and final $(k=155)$ iterations.

(a) Chord length distribution

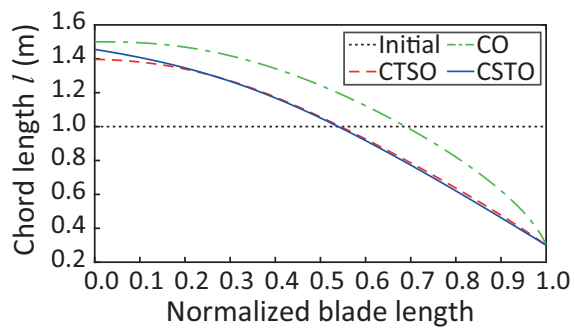

(b) Twist angle distribution

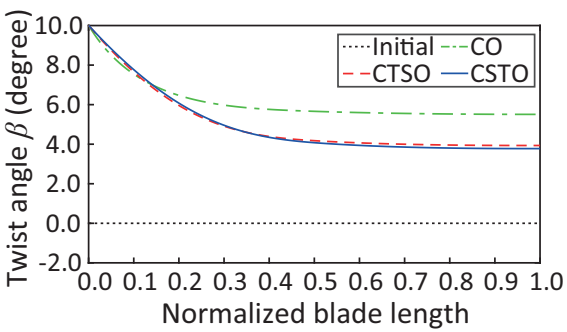

Fig. 5.17. Final distributions of the blade chord length $l$ (a) and twist angle $\beta$ (b) in the longitudinal blade direction for the CO, CTSO and CSTO update schemes. The initial distributions for $l$ and $\beta$ are plotted for comparison.

which it may be concluded that the monolithic approach for the present problem is superior to the two staggered approaches.

\subsection{Conclusions}

This chapter compares the performance of a monolithic and two staggered numerical update schemes for a coupled shape and topology optimization method for thin-walled beam structures. In order to limit the computational time of the optimization method, the beam structures are modeled as 2.5D configurations. Here, 1D beam elements are used to simulate the beam response in the longitudinal direction, while the cross- 


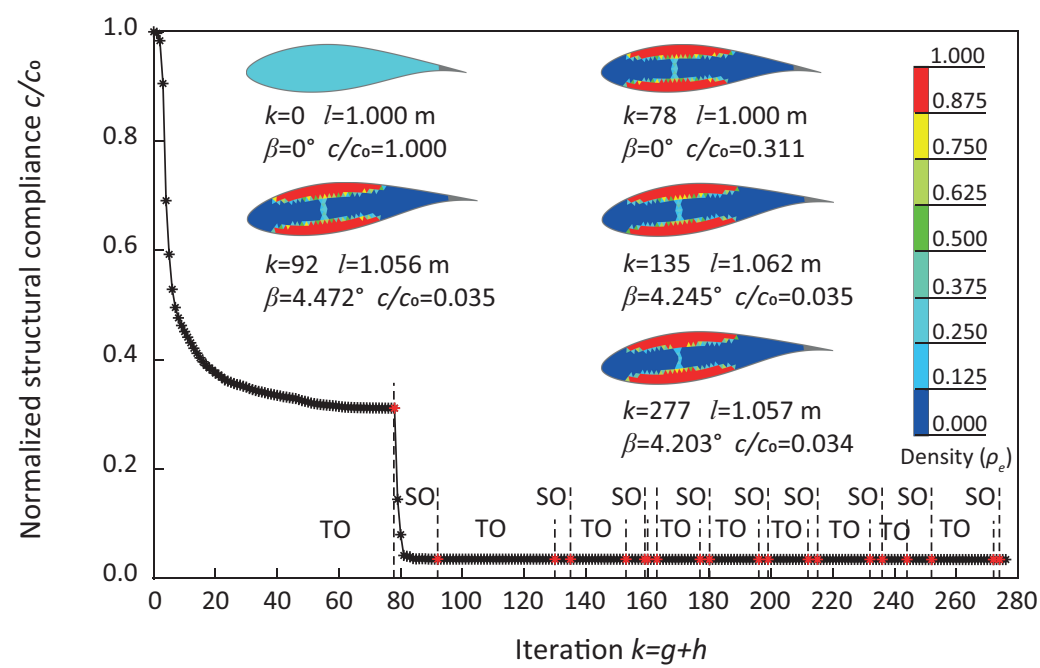

Fig. 5.18. Coupled Topology and Shape Optimization (CTSO): convergence behavior of the normalized compliance $c / c_{0}$ of the HAWT rotor blade. The inset shows the evolution of the 6th cross-section (located at the half-length of the rotor blade) after various numbers of iterations $k$.

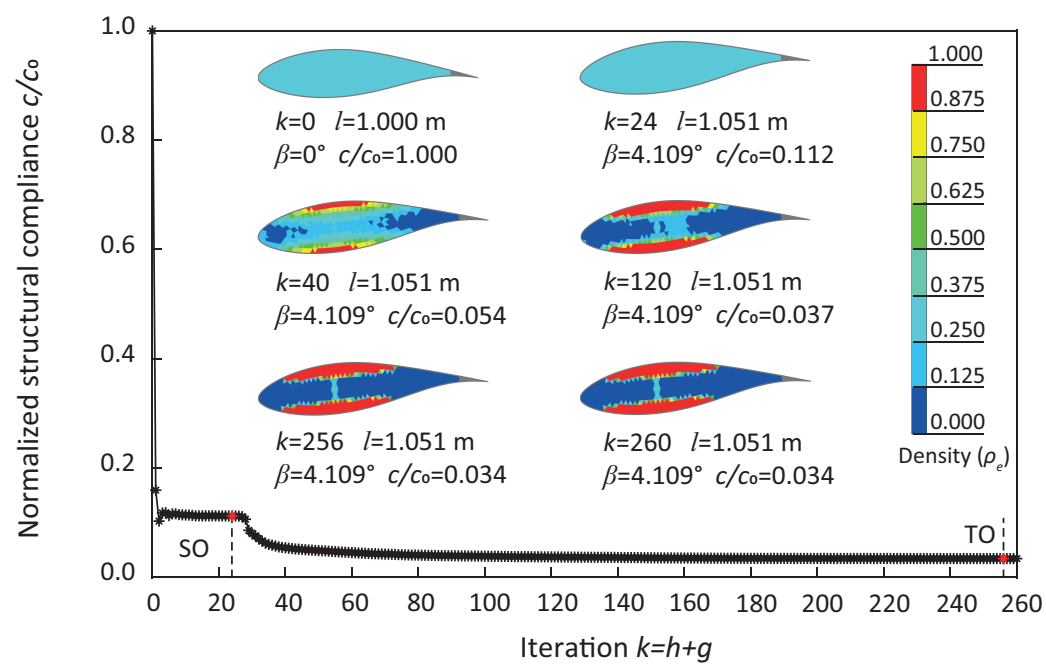

Fig. 5.19. Coupled Shape and Topology Optimization (CSTO): convergence behavior of the normalized compliance $c / c_{0}$ of the HAWT rotor blade. The inset shows the evolution of the 6th cross-section (located at the half-length of the rotor blade) after various numbers of iterations $k$. 
(a) CTSO ( $k=277)$

(b) CSTO $(k=260)$

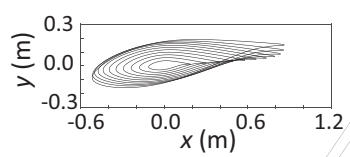

(2) Front view of crosssectional profiles

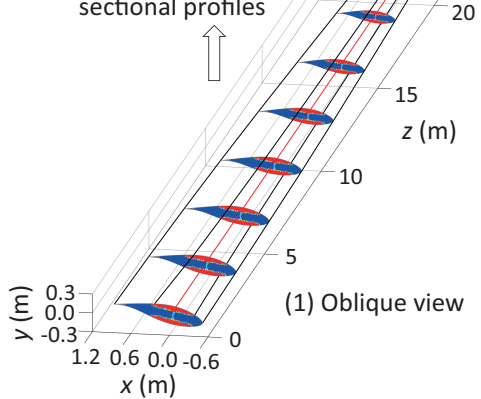

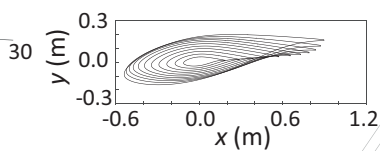

(2) Front view of crosssectional profiles

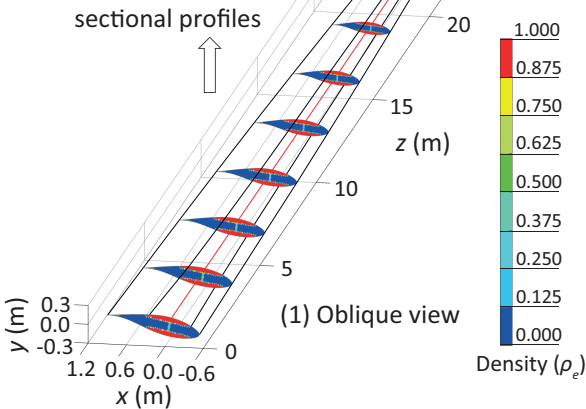

Fig. 5.20. CTSO and CSTO update schemes: blade configuration (outer shape and interior material distribution) after the final $(k=277$ (CTSO) and $k=260$ (CSTO)) iterations.

sectional properties of the beam elements are calculated from additional 2D FEM analyses. The sensitivities with respect to the shape and topology design variables are derived in closed form in order to take advantage of a computationally efficient, gradient-based optimization algorithm.

The performance of the monolithic and staggered update schemes is first analyzed for thin-walled cantilever beams with circular and square cross-sections, subjected to a point load and/or a torsional moment. Subsequently, a practical case study is considered of a horizontal-axis wind turbine (HAWT) rotor blade subjected to representative wind loading conditions. The aerodynamic loading applied on the rotor blade is dependent on the shape of the design domain, whereby the aerodynamic performance is warranted through the formulation of a separate constraint condition on the rotor power coefficient. For the cases analyzed the three different update schemes generally result in comparable design concepts; only occasionally some differences occur, such as the presence of a shear web in the optimized design of the rotor blade, which appeared for the two staggered update schemes but remained absent for the monolithic update scheme. Further, the convergence speed and the structural compliance calculated are rather sensitive to the algorithmic details of the numerical update scheme applied, which is because in shape and topology optimization problems the landscape of solutions typically is characterized by numerous local minima. Despite this sensitivity, for most cases examined the monolithic update scheme provides the lowest structural compliance, which is due to the fact that it typically navigates a larger design space than the two staggered schemes. An exception to this rule follows from the problem of a cantilever beam with a circular design unit subjected to a torsional moment, whereby the monolithic scheme gets stuck in a less optimal, local minimum that ignores the 
fact that under uniform torsion shape optimization (virtually) has no influence on the final, optimized beam configuration. Additionally, the numerical example of the wind turbine blade demonstrates that the coupled optimization approach may serve as a useful tool for improving the aerostructural performance of practical wind turbine systems.

In the present comparison study, the 3D beam configurations analyzed are adequately described by combining a single cross-sectional design unit with length and twist angle parameters that characterize the shape variation in the longitudinal beam direction via 5 NURBS control points. Note, however, that the coupled optimization approach can be naturally extended to more advanced 3D geometries that require a larger number of cross-sectional design units (see e.g., [119]) and/or a larger number of NURBS control points, which may be interesting for a further comparison of the performances of the present numerical update schemes. Additionally, the use of the classical SIMP topology optimization method may occasionally result in the appearance of intermediate element densities, as has been observed for the shear web in the optimized topology computed for a wind turbine blade. In order to reduce the appearance of intermediate densities in topology optimization results, more elaborate strategies, such as the modified SIMP method or the projection method, as presented in [94, 121, 122, can be applied in the coupled shape and topology optimization formulation, which is considered to be another topic for future work. 


\section{Chapter 6}

\section{Conclusions and recommendations}

\subsection{Conclusions}

The research work presented in this thesis aims at the development of a coupled shape and topology optimization framework, which simultaneously optimizes the external shape and interior layout of horizontal-axis wind turbine (HAWT) rotor blades with consideration of both aerodynamic and structural design requirements.

The development of the coupled optimization method for HAWTs has been divided into four parts: (1) formulating a coupled domain shape and topology optimization framework for 2D design problems, and implementing this framework numerically using a staggered update scheme; (2) extending the 2D optimization method for the structural design of $2.5 \mathrm{D}$ and $3 \mathrm{D}$ beam models; (3) applying the $2.5 \mathrm{D}$ coupled optimization method for the aerostructural design of HAWT blades; (4) implementing the coupled optimization method by applying a monolithic update scheme, and investigating the effect of the solution strategy on the optimization results through a comparison with the optimization results following from the staggered numerical update scheme used in tasks (1)-(3). The main conclusions for each of the four research tasks above have been summarized in the sections below.

\subsubsection{Coupled shape and topology optimization method}

A novel modeling framework has been presented that couples topology and domain shape optimization. The shape and topology design variables are incrementally updated in a sequential fashion, using a so-called staggered numerical update scheme. Accordingly, the shape of the design domain is momentarily frozen when performing a topology optimization step, and the topology of the design domain is temporarily kept fixed when carrying out a shape optimization step. The sequence of alternating shape and topology optimization steps is repeated until the value of the objective function meets the convergence criteria. The geometry for domain shape optimiza- 


\subsection{Conclusions}

tion is described by NURBS, in order to allow for the analysis of relatively complex structural forms. The domain shape optimization procedure is performed using an Sequential Quadratic Programming (SQP) method in combination with an efficient gradient-based solution strategy. The topology optimization procedure is carried out in accordance with the SIMP approach.

The usefulness of the coupled optimization approach has been demonstrated by analyzing several benchmark problems. The simulation results confirm a strong dependency on the initial conditions and bounds selected, showing that a careful construction of the design domain generally is necessary and meaningful. Under the specific conditions whereby the initial design domain has a basic, rectangular shape, of which the size is prescribed by a relatively large value, the effect of domain shape optimization on the computational result may be relatively small, due to which the coupled topology and domain shape optimization framework leads to a similar outcome as that obtained by pure topology optimization.

Conversely, the coupled optimization method may find a better optimum than the pure topology optimization method when the maximal design domain is a priori undefined, since in the coupled optimization method the design domain for the topology optimization step is allowed to be changed (enlarged). The coupled optimization method is a suitable method for the optimization of wind turbines, since it straightforwardly facilitates the incorporation of aerodynamic shape optimization criteria commonly applied in the design of wind turbine rotor blades.

\subsubsection{Coupled optimization of beam-type structures}

The coupled optimization framework presented in Section 6.1.1 has been extended to simultaneously optimize the outer shape and the internal topology of beam-type structures using a staggered update procedure. The objective function refers to the structural compliance, which is evaluated using 2.5D and 3D FEM models. In the 2.5D model, standard 2-node beam elements are used along the longitudinal direction of the beam, whereby the cross-sectional properties of the beam elements are calculated from additional 2D FEM analyses. Conversely, in the 3D model the beam geometry is simulated using 3D continuum elements. The shape of the beam-type structures is parameterized using NURBS, with the shape design variables being represented by the NURBS control points. The topology design variables are reflected by the relative densities assigned to each finite element. The design variables are iteratively updated by applying NURBS-based shape and density-based topology optimization techniques, which use analytic shape and topology sensitivities that ensure an accurate and computationally efficient solution procedure.

A comparison study of a cantilever beam problem subjected to pure shape optimization and pure topology optimization illustrates that the $2.5 \mathrm{D}$ and $3 \mathrm{D}$ beam models lead to similar shape and topology designs, but that the 2.5D beam model has a significantly higher computational efficiency. Specifically, the computational times for the 2.5D model are about a factor 70 (shape optimization) and 1.4 (topology optimization) lower than for the 3D model, which indicates that in the coupled optimization approach the optimization of the shape provides the largest contribution to 
the higher computational efficiency of the 2.5D model. The coupled shape and topology optimization analysis subsequently performed on the $2.5 \mathrm{D}$ cantilever beam model demonstrates that the specific order at which the alternating shape and topology optimization increments are performed in the staggered update procedure turns out to have some influence on the final computational result for the boundary value problem considered. Further, the convergence speed for the optimization procedure starting with an incremental topology optimization step appears to be somewhat lower than that resulting from starting with an incremental shape optimization step, although it may be reasonably expected that this feature is problem dependent. Despite these differences, the final beam structures following from the two staggered update schemes illustrate how shape and topology can be efficiently optimized in an integrated, coupled fashion.

\subsubsection{Aerostructural design of turbine blades}

On the basis of the efficient 2.5D coupled optimization framework presented in Section 6.1.2 a coupled multi-objective shape and topology optimization (CMSTO) framework has been proposed for the aerostructural design of HAWT rotor blades. The framework enables to simultaneously optimize the outer shape and the internal structural layout of the turbine blade with respect to aerodynamic and structural requirements. The optimization process is performed on the weighted sum of two design objectives, which are the rotor power coefficient and the structural compliance of the blade. The power coefficient and structural compliance are evaluated in a computationally efficient manner by using so-called BEM and beam FEM models, respectively. The shape design variables are represented by the locations of NURBS control points that govern the blade geometry. The topology design variables are represented by the relative densities assigned to the finite elements modeling the blade cross-sections, in accordance with the SIMP method. The coupled optimization model for a turbine blade has been solved in an incremental-iterative fashion, by alternatively performing shape and topology optimization steps, until the solution satisfies the convergence criterion imposed. Here, the computational efficiency of the numerical procedure is warranted through the use of closed-form sensitivities in a gradient-based algorithm for the CMSTO approach.

The specific features of the CMSTO approach have been demonstrated by comparing the optimized design computed for a NREL 5MW reference rotor blade with the results obtained from a separate Aerodynamic Shape Optimization-Structural Topology Optimization (ASO-STO) approach and a pure Structural Topology Optimization (STO) approach. The optimization results following from the CMSTO approach for different weighting factors indicate that the overall stiffness of a blade typically decreases with an increase of the rotor power coefficient. The rotor power coefficients of the designs generated by the CMSTO approach with a weighting factor $\omega=0.75$ and the ASO-STO approach are about 4\% larger than that of the reference rotor blade. However, the structural compliance of the blade calculated by the CMSTO approach with $\omega=0.75$ is $16 \%$ lower than that computed by ASO-STO approach. The application of the CMSTO approach with the aerodynamic optimization being switched off 
leads to a reduction of the blade structural compliance of approximately $41 \%$ compared to a pure STO approach. Such significant improvements clearly demonstrate the benefits of the CMSTO approach in the quest of wind turbines with higher power output and better structural performance.

\subsubsection{Comparison of numerical update schemes}

For the coupled optimization frameworks presented in Sections 6.1.1 to 6.1.3 the numerical implementation has been based on a staggered numerical update scheme. The numerical implementation of the coupled optimization method may be alternatively based on a monolithic numerical update scheme; the performance of this update scheme is compared with that of staggered update scheme for thin-walled beam structures. In order to limit the computational time of the optimization method, the beam structures are modeled as a 2.5D configuration, see also Section 6.1.2. The sensitivities with respect to the shape and topology design variables are derived in closed form in order to take advantage of a computationally efficient, gradient-based optimization algorithm.

The performance of the monolithic and staggered update schemes is first analyzed for thin-walled cantilever beams with circular and square cross-sections, subjected to a point load and/or a torsional moment. Subsequently, a practical case study is considered of a horizontal-axis wind turbine (HAWT) rotor blade subjected to representative wind loading conditions. The aerodynamic loading applied on the rotor blade is dependent on the shape of the design domain, whereby the aerodynamic performance is warranted through the formulation of a separate constraint condition on the rotor power coefficient. For the cases analyzed the three different update schemes generally result in comparable design concepts; only occasionally some differences occur, such as the presence of a shear web in the optimized design of the rotor blade, which appeared for the two staggered update schemes but remained absent for the monolithic update scheme. Further, the convergence speed and the structural compliance calculated are rather sensitive to the algorithmic details of the numerical update scheme applied, which is because in shape and topology optimization problems the landscape of solutions typically is characterized by numerous local minima. Despite this sensitivity, for most cases examined the monolithic update scheme provides the lowest structural compliance, which is due to the fact that it typically navigates a larger design space than the two staggered schemes. An exception to this rule follows from the problem of a cantilever beam with a circular design unit subjected to a torsional moment, whereby the monolithic scheme gets stuck in a less optimal, local minimum that ignores the fact that under uniform torsion shape optimization (virtually) has no influence on the final, optimized beam configuration. Additionally, the numerical example of the wind turbine blade demonstrates that the coupled optimization approach may serve as a useful tool for improving the aerostructural performance of practical wind turbine systems. 


\subsection{Recommendations}

The current research work focuses on the development of a coupled shape and topology optimization framework for the aerostructural design of HAWT rotor blades. Although the computational results of the problems investigated in this thesis confirm the usefulness and advantages of the proposed method, there are still some issues worthwhile for further study, as listed below.

- In the aerostructural design analyses of wind turbine blades as presented in Chapters 4 and 5 the blade material is assumed to be isotropically elastic. Although this assumption is reasonable, for wind turbine blades made of a composite material, such as a glass fiber reinforced polyester or a glass fiber reinforced epoxy, the directional dependence of the material properties may be accounted for in the optimization procedure in order to further improve the structural performance of wind turbine blades.

- The optimization problems analyzed in this thesis focus on minimizing the blade structural compliance and maximizing the rotor power coefficient, thereby accounting for material volume and geometrical design constraints. It would be also interesting to consider alternative optimization problems, which, for example, focus on simultaneously maximizing the rotor power output and minimizing the blade mass with constraint on the blade tip deflection.

- In the present aerostructural optimization method the blade outer shape is governed by the chord and twist distributions of a standard airfoil (e.g., NACA 64) in the longitudinal blade direction. Although the selection of a specific airfoil profile limits the computational costs of the optimization method, it also restricts the design space. In order to further improve the aerostructural design, the airfoil profile itself may also be optimized during shape optimization. To this end, it is necessary to incorporate an extra aerodynamic solver (e.g., XFoil) into the coupled optimization framework that evaluates the aerodynamic characteristics (i.e., the lift and drag coefficient) of the airfoil. Additionally, a high-fidelity CFD method may be included in the coupled optimization framework for improving the prediction of the aerodynamic forces on the wind turbine blade under complex, turbulent loading conditions. The high computational demand of CFD methods requires the incorporation of high-performance computing techniques, such as parallel computing.

- The presented coupled shape and topology optimization framework uses the FEM method for solving boundary value problems. Although the problems studied address several numerical discretization effects, such as the mesh sensitivity of the numerical result and the possible appearance of unfavorable element distortions, more research on such aspects is necessary in order to apply the coupled optimization method for advanced three-dimensional structures with complex geometries. Along this line, an interesting topic for future research may be to integrate the current optimization framework with an isogeometric, higher-order discretization method that uses an automated mesh generation procedure.

- In the present work, only the aerodynamic loads are considered for the opti- 
mization of wind turbine blades. However, in practice, the loading condition of wind turbine blades is rather complicated, and also includes gravitational loads, operational loads, fatigue loads, etc. Furthermore, the aerodynamic loads are calculated in accordance with the specific wind conditions adopted in this work. It may be expected that the optimized blade geometry (both the external shape and interior layout) changes to some extent when multiple loading cases are considered in the coupled optimization framework. The investigation of this aspect is a topic for future research. 


\section{Bibliography}

[1] P. M. Cox, R. A. Betts, C. D. Jones, S. A. Spall, and I. J. Totterdell. Acceleration of global warming due to carbon-cycle feedbacks in a coupled climate model. Nature, 408(6809):184, 2000.

[2] M. Meinshausen, N. Meinshausen, W. Hare, S. C. B. Raper, K. Frieler, R. Knutti, D. J. Frame, and M. R. Allen. Greenhouse-gas emission targets for limiting global warming to $2{ }^{\circ} \mathrm{C}$. Nature, 458(7242):1158, 2009.

[3] O. Edenhofer, R. Pichs-Madruga, Y. Sokona, E. Farahani, S. Kadner, K. Seyboth, A. Adler, I. Baum, S. Brunner, P. Eickemeier, B. Kriemann, J. Savolainen, S. Schömer, C. von Stechow, T. Zwickel, and J. C. Minx. Summary for Policymakers. In Climate Change 2014: Mitigation of Climate Change. Contribution of Working Group III to the Fifth Assessment Report of the Intergovernmental Panel on Climate Change. Cambridge University Press, 2014.

[4] N. L. Panwar, S. C. Kaushik, and S. Kothari. Role of renewable energy sources in environmental protection: A review. Renewable and Sustainable Energy Reviews, 15(3):1513-1524, 2011.

[5] BP. Statistical review of world energy, 2019.

[6] M. R. Islam, S. Mekhilef, and R. Saidur. Progress and recent trends of wind energy technology. Renewable and Sustainable Energy Reviews, 21:456-468, 2013.

[7] Global Wind Energy Council (GWEC). Global wind report 2018, 2019.

[8] International Energy Agency (IEA). World energy investment 2019, 2019.

[9] WindEurope. Financing and investment trends: The European wind industry in 2018, 2018.

[10] U.S. Department of Energy. 2017 wind technologies market report, 2017.

[11] B. K. Sahu. Wind energy developments and policies in China: A short review. Renewable and Sustainable Energy Reviews, 81:1393-1405, 2018. 
[12] International Energy Agency (IEA). Global energy \& CO2 status report, 2018.

[13] R. S. Amano. Review of wind turbine research in 21st century. Journal of Energy Resources Technology, 139(5):050801, 2017.

[14] P. J. Schubel and R. J. Crossley. Wind turbine blade design. Energies, 5(9): 3425-3449, 2012.

[15] T. Burton, N. Jenkins, D. Sharpe, and E. Bossanyi. Wind energy handbook. John Wiley \& Sons, 2011.

[16] A. Betz. Introduction to the theory of flow machines. (D. G. Randall, Trans.) Oxford: Pergamon Press, 1966.

[17] G. M. J. Herbert, S. Iniyan, E. Sreevalsan, and S. Rajapandian. A review of wind energy technologies. Renewable and Sustainable Energy Reviews, 11(6): 1117-1145, 2007.

[18] M. Shaheen and M. Z. Khan. A method of data mining for selection of site for wind turbines. Renewable and Sustainable Energy Reviews, 55:1225-1233, 2016.

[19] P. Fuglsang and K. Thomsen. Site-specific design optimization of 1.5-2.0 MW wind turbines. Journal of solar energy engineering, 123(4):296-303, 2001.

[20] R. Shakoor, M. Y. Hassan, A. Raheem, and Y.-K. Wu. Wake effect modeling: A review of wind farm layout optimization using Jensen's model. Renewable and Sustainable Energy Reviews, 58:1048-1059, 2016.

[21] S. Rodrigues, P. Bauer, and P. A. N. Bosman. Multi-objective optimization of wind farm layouts - Complexity, constraint handling and scalability. Renewable and Sustainable Energy Reviews, 65:587-609, 2016.

[22] Z. Song, Z. Zhang, and X. Chen. The decision model of 3-dimensional wind farm layout design. Renewable Energy, 85:248-258, 2016.

[23] S. Rehman, Md. M. Alam, L. M. Alhems, and M. M. Rafique. Horizontal axis wind turbine blade design methodologies for efficiency enhancement-A review. Energies, 11(3):506, 2018.

[24] LM WIND POWER. Meet a record-breaker: LM $88.4 \mathrm{P}$-The world's longest blade. https://www.lmwindpower.com/en/products-and-services/ blade-types/longest-blade-in-the-world. [Online; accessed 26-July-2019].

[25] N. Buckney, S. Green, A. Pirrera, and P. M. Weaver. On the structural topology of wind turbine blades. Wind Energy, 16(4):545-560, 2013.

[26] L. Wang, X. Liu, and A. Kolios. State of the art in the aeroelasticity of wind turbine blades: Aeroelastic modelling. Renewable and Sustainable Energy Reviews, 64:195-210, 2016. 
[27] M. B. Ageze, Y. Hu, and H. Wu. Wind turbine aeroelastic modeling: Basics and cutting edge trends. International Journal of Aerospace Engineering, 2017, 2017.

[28] B. Amirzadeh, A. Louhghalam, M. Raessi, and M. Tootkaboni. A computational framework for the analysis of rain-induced erosion in wind turbine blades, part I: Stochastic rain texture model and drop impact simulations. Journal of Wind Engineering and Industrial Aerodynamics, 163:33-43, 2017.

[29] P. S. Veers, T. D. Ashwill, H. J. Sutherland, D. L. Laird, D. W. Lobitz, D. A. Griffin, J. F. Mandell, W. D. Musial, K. Jackson, M. Zuteck, et al. Trends in the design, manufacture and evaluation of wind turbine blades. Wind Energy, 6 (3):245-259, 2003.

[30] L. Fingersh, M. Hand, and A. Laxson. Wind turbine design cost and scaling model. Technical report, National Renewable Energy Lab.(NREL), Golden, CO (United States), 2006.

[31] T. D. Ashwill. Materials and innovations for large blade structures: Research opportunities in wind energy technology. In 50th AIAA/ASME/ASCE/AHS/ASC Structures, Structural Dynamics, and Materials Conference, page 2407, 2009.

[32] C.-J. Bai and W.-C. Wang. Review of computational and experimental approaches to analysis of aerodynamic performance in horizontal-axis wind turbines (HAWTs). Renewable and Sustainable Energy Reviews, 63:506-519, 2016.

[33] H. Snel. Review of aerodynamics for wind turbines. Wind Energy, 6(3):203-211, 2003.

[34] C. Bak. Aerodynamic design of wind turbine rotors. In Advances in wind turbine blade design and materials, pages 59-108. Elsevier, 2013.

[35] C. Pavese, C. Tibaldi, F. Zahle, and T. Kim. Aeroelastic multidisciplinary design optimization of a swept wind turbine blade. Wind Energy, 20(12):1941-1953, 2017.

[36] L. Wang, T. Wang, J. Wu, and G. Chen. Multi-objective differential evolution optimization based on uniform decomposition for wind turbine blade design. Energy, 120:346-361, 2017.

[37] J. X. V. Neto, E. J. G. Junior, S. R. Moreno, H. V. H. Ayala, V. C. Mariani, and L. dos Santos Coelho. Wind turbine blade geometry design based on multiobjective optimization using metaheuristics. Energy, 162:645-658, 2018.

[38] X. Shen, J.-G. Chen, X.-C. Zhu, P.-Y. Liu, and Z.-H. Du. Multi-objective optimization of wind turbine blades using lifting surface method. Energy, 90: 1111-1121, 2015. 
[39] A. Dal Monte, S. De Betta, M. R. Castelli, and E. Benini. Proposal for a coupled aerodynamic-structural wind turbine blade optimization. Composite Structures, 159:144-156, 2017.

[40] A. Dal Monte, M. R. Castelli, and E. Benini. Multi-objective structural optimization of a HAWT composite blade. Composite Structures, 106:362-373, 2013.

[41] E. M. Fagan, O. De La Torre, S. B. Leen, and J. Goggins. Validation of the multiobjective structural optimisation of a composite wind turbine blade. Composite Structures, 204:567-577, 2018.

[42] A. Chehouri, R. Younes, A. Ilinca, and J. Perron. Review of performance optimization techniques applied to wind turbines. Applied Energy, 142:361-388, 2015 .

[43] M. P. Bendsoe and O. Sigmund. Topology Optimization: Theory, Methods and Applications. Springer, Berlin, 2003.

[44] M. Jureczko, M. Pawlak, and A. Mężyk. Optimisation of wind turbine blades. Journal of Materials Processing Technology, 167(2-3):463-471, 2005.

[45] C. L. Bottasso, F. Campagnolo, A. Croce, S. Dilli, F. Gualdoni, and M. B. Nielsen. Structural optimization of wind turbine rotor blades by multilevel sectional/multibody/3D-FEM analysis. Multibody System Dynamics, 32(1):87$116,2014$.

[46] R. H. Barnes and E. V. Morozov. Structural optimisation of composite wind turbine blade structures with variations of internal geometry configuration. Composite Structures, 152:158-167, 2016.

[47] J. Sjølund and E. Lund. Structural gradient based sizing optimization of wind turbine blades with fixed outer geometry. Composite Structures, 203:725-739, 2018 .

[48] Y.-L. Hsu. A review of structural shape optimization. Computers in Industry, 25(1):3-13, 1994.

[49] X. Shen, H. Yang, J. Chen, X. Zhu, and Z. Du. Aerodynamic shape optimization of non-straight small wind turbine blades. Energy Conversion and Management, 119:266-278, 2016.

[50] M. Tahani, G. Kavari, M. Masdari, and M. Mirhosseini. Aerodynamic design of horizontal axis wind turbine with innovative local linearization of chord and twist distributions. Energy, 131:78-91, 2017.

[51] O. Sigmund and K. Maute. Topology optimization approaches. Structural and Multidisciplinary Optimization, 48(6):1031-1055, 2013. 
[52] J. D. Deaton and R. V. Grandhi. A survey of structural and multidisciplinary continuum topology optimization: post 2000. Structural and Multidisciplinary Optimization, 49(1):1-38, 2014.

[53] N. Buckney, S. Green, A. Pirrera, and P. M. Weaver. On the structural topology of wind turbine blades. Wind Energy, 16(4):545-560, 2013.

[54] I. Y. Kim and B. M. Kwak. Design space optimization using a numerical design continuation method. International Journal for Numerical Methods in Engineering, 53(8):1979-2002, 2002.

[55] W. J. Zhu, W. Z. Shen, and J. N. Sørensen. Integrated airfoil and blade design method for large wind turbines. Renewable Energy, 70:172-183, 2014.

[56] D. Vučina, I. Marinić-Kragić, and Z. Milas. Numerical models for robust shape optimization of wind turbine blades. Renewable Energy, 87:849-862, 2016.

[57] F. M. Jensen and K. Branner. Introduction to wind turbine blade design. In Advances in wind turbine blade design and materials, pages 3-28. Elsevier, 2013.

[58] R. Barrett and A. Ning. Integrated free-form method for aerostructural optimization of wind turbine blades. Wind Energy, 21(8):663-675, 2018.

[59] A. Pourrajabian, P. A. N. Afshar, M. Ahmadizadeh, and D. Wood. Aerostructural design and optimization of a small wind turbine blade. Renewable Energy, 87:837-848, 2016.

[60] H. Yang, J. Chen, X. Pang, and G. Chen. A new aero-structural optimization method for wind turbine blades used in low wind speed areas. Composite Structures, 207:446-459, 2019.

[61] A. Ning and D. Petch. Integrated design of downwind land-based wind turbines using analytic gradients. Wind Energy, 19(12):2137-2152, 2016.

[62] S. Garreau, P. Guillaume, and M. Masmoudi. The topological asymptotic for PDE systems: the elasticity case. SIAM Journal on Control and Optimization, 39(6):1756-1778, 2001.

[63] N. P. van Dijk, K. Maute, M. Langelaar, and F. Van Keulen. Level-set methods for structural topology optimization: a review. Structural and Multidisciplinary Optimization, 48(3):437-472, 2013.

[64] X. Huang and Y.-M. Xie. A further review of ESO type methods for topology optimization. Structural and Multidisciplinary Optimization, 41(5):671-683, 2010.

[65] W. Zhang, L. Zhao, T. Gao, and S. Cai. Topology optimization with closed B-splines and Boolean operations. Computer Methods in Applied Mechanics and Engineering, 315:652-670, 2017. 
[66] O. Sigmund. A 99 line topology optimization code written in Matlab. Structural and Multidisciplinary Optimization, 21(2):120-127, 2001.

[67] E. Andreassen, A. Clausen, M. Schevenels, B. S. Lazarov, and O. Sigmund. Efficient topology optimization in MATLAB using 88 lines of code. Structural and Multidisciplinary Optimization, 43(1):1-16, 2011.

[68] K. Liu and A. Tovar. An efficient 3D topology optimization code written in Matlab. Structural and Multidisciplinary Optimization, 50(6):1175-1196, 2014.

[69] S. Rojas-Labanda and M. Stolpe. Benchmarking optimization solvers for structural topology optimization. Structural and Multidisciplinary Optimization, 52 (3):527-547, 2015.

[70] S. Rojas-Labanda and M. Stolpe. An efficient second-order SQP method for structural topology optimization. Structural and Multidisciplinary Optimization, 53(6):1315-1333, 2016.

[71] N. Aage, E. Andreassen, B. S. Lazarov, and O. Sigmund. Giga-voxel computational morphogenesis for structural design. Nature, 550(7674):84, 2017.

[72] I. G. Jang and B. M. Kwak. Design space optimization using design space adjustment and refinement. Structural and Multidisciplinary optimization, 35 (1):41-54, 2008 .

[73] S. Osher and J. A. Sethian. Fronts propagating with curvature-dependent speed: algorithms based on Hamilton-Jacobi formulations. Journal of Computational Physics, 79(1):12-49, 1988.

[74] Z. Luo, L. Tong, M. Y. Wang, and S. Wang. Shape and topology optimization of compliant mechanisms using a parameterization level set method. Journal of Computational Physics, 227(1):680-705, 2007.

[75] S. Y. Wang, K. M. Lim, B. C. Khoo, and M. Y. Wang. An extended level set method for shape and topology optimization. Journal of Computational Physics, 221(1):395-421, 2007.

[76] J. Zhang, W. H. Zhang, J. H. Zhu, and L. Xia. Integrated layout design of multicomponent systems using XFEM and analytical sensitivity analysis. Computer Methods in Applied Mechanics and Engineering, 245:75-89, 2012.

[77] Y. Wang and Z. Kang. A velocity field level set method for shape and topology optimization. International Journal for Numerical Methods in Engineering, 115 (11):1315-1336, 2018.

[78] G. Allaire and F. Jouve. Coupling the level set method and the topological gradient in structural optimization. In IUTAM symposium on Topological Design Optimization of Structures, Machines and Materials, pages 3-12. Springer, 2006. 
[79] A. Takezawa, S. Nishiwaki, and M. Kitamura. Shape and topology optimization based on the phase field method and sensitivity analysis. Journal of Computational Physics, 229(7):2697-2718, 2010.

[80] A. L. Gain and G. H. Paulino. Phase-field based topology optimization with polygonal elements: a finite volume approach for the evolution equation. Structural and Multidisciplinary Optimization, 46(3):327-342, 2012.

[81] S. H. Jeong, G. H. Yoon, A. Takezawa, and D.-H. Choi. Development of a novel phase-field method for local stress-based shape and topology optimization. Computers $\mathcal{E}$ Structures, 132:84-98, 2014.

[82] X. Guo, W. Zhang, and W. Zhong. Doing topology optimization explicitly and geometrically - a new moving morphable components based framework. Journal of Applied Mechanics, 81(8), 2014.

[83] W. Zhang, J. Yuan, J. Zhang, and X. Guo. A new topology optimization approach based on moving morphable components (MMC) and the ersatz material model. Structural and Multidisciplinary Optimization, 53(6):1243-1260, 2016.

[84] K. Maute and E. Ramm. Adaptive topology optimization. Structural Optimization, 10(2):100-112, 1995.

[85] K. Maute and E. Ramm. Adaptive topology optimization of shell structures. AIAA Journal, 35(11):1767-1773, 1997.

[86] K. Maute, S. Schwarz, and E. Ramm. Adaptive topology optimization of elastoplastic structures. Structural Optimization, 15(2):81-91, 1998.

[87] S. Schwarz, K. Maute, and E. Ramm. Topology and shape optimization for elastoplastic structural response. Computer Methods in Applied Mechanics and Engineering, 190(15-17):2135-2155, 2001.

[88] R. Ansola, J. Canales, J. A. Tarrago, and J. Rasmussen. On simultaneous shape and material layout optimization of shell structures. Structural and Multidisciplinary Optimization, 24(3):175-184, 2002.

[89] L. Piegl and W. Tiller. The NURBS book. Springer, Berlin, 1995.

[90] A.-V. Vuong, Ch. Heinrich, and B. Simeon. ISOGAT: A 2D tutorial MATLAB code for isogeometric analysis. Computer Aided Geometric Design, 27(8):644$655,2010$.

[91] W. Zhang, D. Wang, and J. Yang. A parametric mapping method for curve shape optimization on 3D panel structures. International Journal for Numerical Methods in Engineering, 84(4):485-504, 2010.

[92] T. J. R. Hughes, J. A. Cottrell, and Y. Bazilevs. Isogeometric analysis: CAD, finite elements, NURBS, exact geometry and mesh refinement. Computer Methods in Applied Mechanics and Engineering, 194(39-41):4135-4195, 2005. 
[93] M. P. Bendsøe and O. Sigmund. Material interpolation schemes in topology optimization. Archive of Applied Mechanics, 69(9-10):635-654, 1999.

[94] O. Sigmund. Morphology-based black and white filters for topology optimization. Structural and Multidisciplinary Optimization, 33(4-5):401-424, 2007.

[95] J. Nocedal and S. Wright. Numerical optimization. Springer, New York, 2006.

[96] W. A. Wall, M. A. Frenzel, and C. Cyron. Isogeometric structural shape optimization. Computer Methods in Applied Mechanics and Engineering, 197(33): 2976-2988, 2008.

[97] X. Qian. Full analytical sensitivities in NURBS based isogeometric shape optimization. Computer Methods in Applied Mechanics and Engineering, 199(29): 2059-2071, 2010.

[98] O. Sigmund and J. Petersson. Numerical instabilities in topology optimization: A survey on procedures dealing with checkerboards, mesh-dependencies and local minima. Structural Optimization, 16(1):68-75, 1998.

[99] P. Liu, Y. Luo, and Z. Kang. Multi-material topology optimization considering interface behavior via XFEM and level set method. Computer Methods in Applied Mechanics and Engineering, 308:113-133, 2016.

[100] L. Noël, P. Duysinx, and K. Maute. Level set topology optimization considering damage. Structural and Multidisciplinary Optimization, 56(4):737-753, 2017.

[101] X. Guo, W. Zhang, and W. Zhong. Stress-related topology optimization of continuum structures involving multi-phase materials. Computer Methods in Applied Mechanics and Engineering, 268:632-655, 2014.

[102] L. S. Duarte, W. Celes, A. Pereira, I. F. M. Menezes, and G. H. Paulino. PolyTop++: an efficient alternative for serial and parallel topology optimization on CPUs \& GPUs. Structural and Multidisciplinary Optimization, 52(5):845-859, 2015 .

[103] H. Lian, A. N. Christiansen, D. A. Tortorelli, O. Sigmund, and N. Aage. Combined shape and topology optimization for minimization of maximal von Mises stress. Structural and Multidisciplinary Optimization, 55(5):1541-1557, 2017.

[104] Q. X. Lieu and J. Lee. Multiresolution topology optimization using isogeometric analysis. International Journal for Numerical Methods in Engineering, 112(13): 2025-2047, 2017.

[105] R. Picelli, S. Townsend, C. Brampton, J. Norato, and H. A. Kim. Stress-based shape and topology optimization with the level set method. Computer Methods in Applied Mechanics and Engineering, 329:1-23, 2018. 
[106] K. Dems. Multiparameter shape optimization of elastic bars in torsion. International Journal for Numerical Methods in Engineering, 15(10):1517-1539, 1980.

[107] U. Schramm and W. D. Pilkey. Optimal shape design for thin-walled beam cross-sections. International Journal for Numerical Methods in Engineering, 37 (23):4039-4058, 1994.

[108] P. Vinot, S. Cogan, and J. Piranda. Shape optimization of thin-walled beam-like structures. Thin-Walled Structures, 39(7):611-630, 2001.

[109] V. Apostol, J. L. T. Santos, and M. Paiva. Sensitivity analysis and optimization of truss/beam components of arbitrary cross-section II. Shear stresses. Computers $\&$ Structures, 80(5):391-401, 2002.

[110] T. M. Leung and D. W. Zingg. Aerodynamic shape optimization of wings using a parallel Newton-Krylov approach. AIAA Journal, 50(3):540-550, 2012.

[111] X. Han and D. W. Zingg. An adaptive geometry parametrization for aerodynamic shape optimization. Optimization and Engineering, 15(1):69-91, 2014.

[112] Z. Wang, A. S. J. Suiker, H. Hofmeyer, I. Kalkman, and B. Blocken. Sequentially coupled gradient-based topology and domain shape optimization. $O p$ timization and Engineering (In press), 2020. doi: https://doi.org/10.1007/ s11081-020-09546-3.

[113] V. Giavotto, M. Borri, P. Mantegazza, G. Ghiringhelli, V. Carmaschi, G. C. Maffioli, and F. Mussi. Anisotropic beam theory and applications. Computers E Structures, 16(1-4):403-413, 1983.

[114] G. L. Ghiringhelli and P. Mantegazza. Linear, straight and untwisted anisotropic beam section properties from solid finite elements. Composites Engineering, 4 (12):1225-1239, 1994.

[115] J. P. Blasques and M. Stolpe. Multi-material topology optimization of laminated composite beam cross sections. Composite Structures, 94(11):3278-3289, 2012.

[116] J. P. Blasques. Multi-material topology optimization of laminated composite beams with eigenfrequency constraints. Composite Structures, 111:45-55, 2014.

[117] J. P. Blasques, R. D. Bitsche, V. Fedorov, and B. S. Lazarov. Accuracy of an efficient framework for structural analysis of wind turbine blades. Wind Energy, 19(9):1603-1621, 2016.

[118] J. P. Blasques, M. Stolpe, C. Berggreen, and K. Branner. Optimal design of laminated composite beams. PhD thesis, Technical University of Denmark (DTU), 2011. 
[119] Z. Wang, A. S. J. Suiker, H. Hofmeyer, T. van Hooff, and B. Blocken. Coupled aerostructural shape and topology optimization of horizontal-axis wind turbine rotor blades. Energy Conversion and Management, 212(112621), 2020. doi: https://doi.org/10.1016/j.enconman.2020.112621.

[120] Z. Wang, A. S. J. Suiker, H. Hofmeyer, T. van Hooff, and B. Blocken. Optimization of thin-walled beam structures: Monolithic versus staggered solution schemes. Thin-walled Structures (In press), 2020. doi: https://doi.org/10.1016/ j.tws.2020.107182.

[121] F. Wang, B. S. Lazarov, and O. Sigmund. On projection methods, convergence and robust formulations in topology optimization. Structural and Multidisciplinary Optimization, 43(6):767-784, 2011.

[122] J. K. Guest, J. H. Prévost, and T. Belytschko. Achieving minimum length scale in topology optimization using nodal design variables and projection functions. International Journal for Numerical Methods in Engineering, 61(2):238$254,2004$.

[123] M. O. L. Hansen, J. N. Sørensen, S. Voutsinas, N. Sørensen, and H. Aa. Madsen. State of the art in wind turbine aerodynamics and aeroelasticity. Progress in Aerospace Sciences, 42(4):285-330, 2006.

[124] F. Geng, I. Kalkman, A. S. J. Suiker, and B. Blocken. Sensitivity analysis of airfoil aerodynamics during pitching motion at a Reynolds number of $1.35 \times 10^{5}$. Journal of Wind Engineering and Industrial Aerodynamics, 183:315-332, 2018.

[125] A. Rezaeiha, H. Montazeri, and B. Blocken. Characterization of aerodynamic performance of vertical axis wind turbines: Impact of operational parameters. Energy Conversion and Management, 169:45-77, 2018.

[126] A. Rezaeiha, H. Montazeri, and B. Blocken. Towards optimal aerodynamic design of vertical axis wind turbines: Impact of solidity and number of blades. Energy, 165:1129-1148, 2018.

[127] B.-C. Chen and N. Kikuchi. Topology optimization with design-dependent loads. Finite Elements in Analysis and Design, 37(1):57-70, 2001.

[128] O. Sigmund and P. M. Clausen. Topology optimization using a mixed formulation: An alternative way to solve pressure load problems. Computer Methods in Applied Mechanics and Engineering, 196(13-16):1874-1889, 2007.

[129] E. Lee and J. R. R. A. Martins. Structural topology optimization with designdependent pressure loads. Computer Methods in Applied Mechanics and Engineering, 233:40-48, 2012.

[130] R. T. Marler and J. S. Arora. Survey of multi-objective optimization methods for engineering. Structural and Multidisciplinary Optimization, 26(6):369-395, 2004 . 
[131] Z. Wang, A. S. J. Suiker, H. Hofmeyer, T. van Hooff, and B. Blocken. Sequentially coupled shape and topology optimization for $2.5 \mathrm{D}$ and $3 \mathrm{D}$ beam-type structures. Submitted, 2020.

[132] S. A. Ning. CCBlade. Technical report, National Renewable Energy Laboratory (NREL), Golden, CO (United States), 2013.

[133] J. Jonkman, S. Butterfield, W. Musial, and G. Scott. Definition of a 5-MW reference wind turbine for offshore system development. National Renewable Energy Laboratory, Golden, CO, Technical Report No. NREL/TP-500-38060, 2009.

[134] B. R. Resor. Definition of a $5 \mathrm{MW} / 61.5 \mathrm{~m}$ wind turbine blade reference model. Albuquerque, New Mexico, USA, Sandia National Laboratories, SAND2013-2569 2013, 2013.

[135] K. Takizawa, B. Henicke, T. E. Tezduyar, M.-C. Hsu, and Y. Bazilevs. Stabilized space-time computation of wind-turbine rotor aerodynamics. Computational Mechanics, 48(3):333-344, 2011.

[136] S. A. Ning. A simple solution method for the blade element momentum equations with guaranteed convergence. Wind Energy, 17(9):1327-1345, 2014.

[137] J. P. Monteiro, M. R. Silvestre, H. Piggott, and J. C. Andre. Wind tunnel testing of a horizontal axis wind turbine rotor and comparison with simulations from two blade element momentum codes. Journal of Wind Engineering and Industrial Aerodynamics, 123:99-106, 2013.

[138] B. Plaza, R. Bardera, and S. Visiedo. Comparison of BEM and CFD results for MEXICO rotor aerodynamics. Journal of Wind Engineering and Industrial Aerodynamics, 145:115-122, 2015.

[139] Y. Kim, H. A. Madsen, M. Aparicio-Sanchez, G. Pirrung, and T. Lutz. Assessment of blade element momentum codes under varying turbulence levels by comparing with blade resolved computational fluid dynamics. Renewable Energy, 160:788-802, 2020.

[140] L. A. Viterna and D. C. Janetzke. Theoretical and experimental power from large horizontal-axis wind turbines. Technical report, National Aeronautics and Space Administration, Cleveland, OH (USA). Lewis Research Center, 1982.

[141] D. Hu, O. Hua, and Z. Du. A study on stall-delay for horizontal axis wind turbine. Renewable Energy, 31(6):821-836, 2006.

[142] Z. Du and M. Selig. A 3-D stall-delay model for horizontal axis wind turbine performance prediction. In 1998 ASME Wind Energy Symposium, page 21, 1998. 
[143] V. Esfahanian, A. S. Pour, I. Harsini, A. Haghani, R. Pasandeh, A. Shahbazi, and G. Ahmadi. Numerical analysis of flow field around NREL phase II wind turbine by a hybrid CFD/BEM method. Journal of Wind Engineering and Industrial Aerodynamics, 120:29-36, 2013.

[144] H. Yang, W. Shen, H. Xu, Z. Hong, and C. Liu. Prediction of the wind turbine performance by using BEM with airfoil data extracted from CFD. Renewable Energy, 70:107-115, 2014.

[145] D. P. Rommel, D. Di Maio, and T. Tinga. Calculating wind turbine component loads for improved life prediction. Renewable energy, 146:223-241, 2020.

[146] M. O. L. Hansen. Aerodynamics of wind turbines. Earthscan, 2008.

[147] M. T. M. Emmerich and A. H. Deutz. A tutorial on multiobjective optimization: fundamentals and evolutionary methods. Natural Computing, 17(3):585-609, 2018 .

[148] O. Sigmund. On the usefulness of non-gradient approaches in topology optimization. Structural and Multidisciplinary Optimization, 43(5):589-596, 2011.

[149] P. Bertolini, M. A. Eder, L. Taglialegne, and P. S. Valvo. Stresses in constant tapered beams with thin-walled rectangular and circular cross sections. ThinWalled Structures, 137:527-540, 2019.

[150] P. Sharafi, L. H. Teh, and M. N. S. Hadi. Shape optimization of thin-walled steel sections using graph theory and ACO algorithm. Journal of Constructional Steel Research, 101:331-341, 2014.

[151] G. Cheng, N. Yu, and N. Olhoff. Optimum design of thermally loaded beamcolumns for maximum vibration frequency or buckling temperature. International Journal of Solids and Structures, 66:20-34, 2015.

[152] Z. Lyu, G. K. W. Kenway, and J. R. R. A. Martins. Aerodynamic shape optimization investigations of the common research model wing benchmark. AIAA Journal, 53(4):968-985, 2015.

[153] G. R. Fischer, T. Kipouros, and A. M. Savill. Multi-objective optimisation of horizontal axis wind turbine structure and energy production using aerofoil and blade properties as design variables. Renewable Energy, 62:506-515, 2014.

[154] Y. Y. Kim and T. S. Kim. Topology optimization of beam cross sections. International Journal of Solids and Structures, 37(3):477-493, 2000.

[155] K. Maute and M. Allen. Conceptual design of aeroelastic structures by topology optimization. Structural and Multidisciplinary Optimization, 27(1-2):27-42, 2004 . 
[156] P. D. Dunning, B. K. Stanford, and H. A. Kim. Coupled aerostructural topology optimization using a level set method for 3D aircraft wings. Structural and Multidisciplinary Optimization, 51(5):1113-1132, 2015.

[157] H. Li, P. Li, L. Gao, L. Zhang, and T. Wu. A level set method for topological shape optimization of 3D structures with extrusion constraints. Computer Methods in Applied Mechanics and Engineering, 283:615-635, 2015.

[158] H.-D. Nguyen, G.-W. Jang, D.-M. Kim, and Y. Y. Kim. Finite prism method based topology optimization of beam cross section for buckling load maximization. Structural and Multidisciplinary Optimization, 57(1):55-70, 2018.

[159] J. Liu, Q. Li, S. Liu, and L. Tong. Dynamic topology optimization design of rotating beam cross-section with gyroscopic effects. Structural and Multidisciplinary Optimization, 58(4):1467-1487, 2018.

[160] N. Bahramian and A. Khalkhali. Crashworthiness topology optimization of thinwalled square tubes, using modified Bidirectional Evolutionary Structural Optimization approach. Thin-Walled Structures, 147:106524, 2020.

[161] K. D. Tsavdaridis, E. Efthymiou, A. Adugu, J. A. Hughes, and L. Grekavicius. Application of structural topology optimisation in aluminium cross-sectional design. Thin-Walled Structures, 139:372-388, 2019.

[162] W. A. Timmer and R. P. J. O. M. van Rooij. Summary of the Delft University wind turbine dedicated airfoils. Journal of Solar Energy EngineeringTransactions of The ASME, 125(4):488-496, 2003. 


\section{List of publications}

\section{Journal papers}

1. Wang, Z., Suiker, A. S. J., Hofmeyer, H., van Hooff, T., Blocken, B. (2020). Coupled aerostructural shape and topology optimization of horizontal-axis wind turbine rotor blades. Energy Conversion and Management, 212, 112621.

2. Wang, Z., Suiker, A. S. J., Hofmeyer, H., Kalkman, I., Blocken, B. (2020). Sequentially coupled gradient-based topology and domain shape optimization. Optimization and Engineering, 34 pages, https://doi.org/10.1007/s11081-02009546-3.

3. Wang, Z., Suiker, A. S. J., Hofmeyer, H., van Hooff, T., Blocken, B. (2020). Optimization of thin-walled beam structures: Monolithic versus staggered solution schemes. Thin-walled Structures, in press, https://doi.org/10.1016/j.tws.2020. 107182 .

4. Wang, Z., Suiker, A. S. J., Hofmeyer, H., van Hooff, T., Blocken, B. (2020). Sequentially coupled shape and topology optimization for $2.5 \mathrm{D}$ and $3 \mathrm{D}$ beam models, under review.

\section{Conference contributions}

1. Wang, Z., Peeters, D. M. J., De Breuker, R. (2020). Aeroelastic optimisation of manufacturable tow-steered composite wings with cruise shape constraint and gust loads. Proceedings of the 10th EASN Virtual International Conference on Innovation in Aviation \& Space to the Satisfaction of the European Citizens.

2. Wang, Z., Suiker, A. S. J., Hofmeyer, H., van Hooff, T., Blocken, B. (2019). Coupled aero-structural shape and topology optimization of HAWT rotor blades. Oral presentation at 13th World Congress of Structural and Multidisciplinary Optimization, Beijing, China.

3. Wang, Z., Suiker, A. S. J., Hofmeyer, H., van Hooff, T., Blocken, B. (2019). Coupled aero-structural shape and topology optimization of horizontal-axis wind 
turbine rotor blades. Poster presentation at 22nd Engineering Mechanics Symposium, Arnhem, Netherlands.

4. Wang, Z., Suiker, A. S. J., Hofmeyer, H., van Hooff, T., Blocken, B. (2018). A sequentially coupled shape and topology optimization method. Oral and poster presentations at 13th World Congress on Computational Mechanics, New York, United States.

5. Wang, Z., Suiker, A. S. J., Hofmeyer, H., Kalkman, I., Blocken, B. (2017). A coupled gradient-based shape and topology optimization method. Poster presentation at 20th Engineering Mechanics Symposium, Arnhem, Netherlands.

6. Wang, Z., Suiker, A. S. J., Blocken, B., Hofmeyer, H., Kalkman, I. (2016). Advanced optimization of horizontal-axis wind turbine rotor blades. Poster presentation at 19th Engineering Mechanics Symposium, Arnhem, Netherlands. 


\section{Curriculum Vitae}

Zhijun Wang was born on the $11^{\text {th }}$ of October 1988 in Sichuan, China. After finishing his Bachelor's degree in Mechatronic Engineering in 2011 at the Xi'an Technological University in China, he started his master studies in Mechanical Design and Theory at the Chongqing University, China. In June 2014 he obtained his Master's degree with a thesis on the vibration simulation and optimization of a vertical mill reducer. Subsequently, he went to industry for one year to work as a R\&D engineer. Upon receiving a scholarship from the China Scholarship Council, in September 2015 he started to work on a Ph.D. project in the groups of Applied Mechanics and Building Physics at the Eindhoven University of Technology in the Netherlands. The results of his Ph.D. research are presented in this thesis. Currently, Zhijun is working as a postdoctoral researcher at the Faculty of Aerospace Engineering of the Delft University of Technology in the Netherlands. His ongoing research is about aeroelastic optimization of manufacturable tow-steered composite aircraft wings with cruise shape constraint and gust loads. 
Bouwstenen is een publicatiereeks van de Faculteit Bouwkunde,

Technische Universiteit Eindhoven.

Zij presenteert resultaten van

onderzoek en andere activiteiten op

het vakgebied der Bouwkunde,

uitgevoerd in het kader van deze

Faculteit.

Bouwstenen en andere proefschriften van de TU/e zijn online beschikbaar via:

https://research.tue.nl/ 
Reeds verschenen in de serie

Bouwstenen

nr 1

Elan: A Computer Model for Building Energy Design: Theory and Validation Martin H. de Wit

H.H. Driessen

R.M.M. van der Velden

nr 2

Kwaliteit, Keuzevrijheid en Kosten:

Evaluatie van Experiment Klarendal, Arnhem

J. Smeets

C. le Nobel

M. Broos

J. Frenken

A. v.d. Sanden

nr 3

Crooswijk:

Van 'Bijzonder' naar 'Gewoon'

Vincent Smit

Kees Noort

$\mathrm{nr} 4$

Staal in de Woningbouw

Edwin J.F. Delsing

nr 5

Mathematical Theory of Stressed

Skin Action in Profiled Sheeting with Various Edge Conditions

Andre W.A.M.J. van den Bogaard

nr 6

Hoe Berekenbaar en Betrouwbaar is

de Coëfficiënt $k$ in $x$-ksigma en $x$-ks?

K.B. Lub

A.J. Bosch

nr 7

Het Typologisch Gereedschap:

Een Verkennende Studie Omtrent

Typologie en Omtrent de Aanpak

van Typologisch Onderzoek

J.H. Luiten

nr 8

Informatievoorziening en Beheerprocessen

A. Nauta

Jos Smeets (red.)

Helga Fassbinder (projectleider)

Adrie Proveniers

J.v.d. Moosdijk nr 9

Strukturering en Verwerking van Tijdgegevens voor de Uitvoering van Bouwwerken

ir. W.F. Schaefer

P.A. Erkelens

nr 10

Stedebouw en de Vorming van een Speciale Wetenschap

K. Doevendans

nr 11

Informatica en Ondersteuning van Ruimtelijke Besluitvorming

G.G. van der Meulen

nr 12

Staal in de Woningbouw, Korrosie-Bescherming van de Begane Grondvloer

Edwin J.F. Delsing

nr 13

Een Thermisch Model voor de

Berekening van Staalplaatbetonvloeren onder Brandomstandigheden

A.F. Hamerlinck

nr 14

De Wijkgedachte in Nederland:

Gemeenschapsstreven in een

Stedebouwkundige Context

K. Doevendans

R. Stolzenburg

nr 15

Diaphragm Effect of Trapezoidally

Profiled Steel Sheets:

Experimental Research into the

Influence of Force Application

Andre W.A.M.J. van den Bogaard

nr 16

Versterken met Spuit-Ferrocement:

Het Mechanische Gedrag van met

Spuit-Ferrocement Versterkte

Gewapend Betonbalken

K.B. Lubir

M.C.G. van Wanroy 
nr 17

De Tractaten van

Jean Nicolas Louis Durand

G. van Zeyl

nr 18

Wonen onder een Plat Dak:

Drie Opstellen over Enkele

Vooronderstellingen van de

Stedebouw

K. Doevendans

nr 19

Supporting Decision Making Processes:

A Graphical and Interactive Analysis of

Multivariate Data

W. Adams

nr 20

Self-Help Building Productivity:

A Method for Improving House Building by Low-Income Groups Applied to Kenya 1990-2000

P. A. Erkelens

nr 21

De Verdeling van Woningen:

Een Kwestie van Onderhandelen

Vincent Smit

nr 22

Flexibiliteit en Kosten in het Ontwerpproces:

Een Besluitvormingondersteunend Model

M. Prins

nr 23

Spontane Nederzettingen Begeleid:

Voorwaarden en Criteria in Sri Lanka

Po Hin Thung

nr 24

Fundamentals of the Design of

Bamboo Structures

Oscar Arce-Villalobos

nr 25

Concepten van de Bouwkunde

M.F.Th. Bax (red.)

H.M.G.J. Trum (red.)

nr 26

Meaning of the Site

Xiaodong Li nr 27

Het Woonmilieu op Begrip Gebracht:

Een Speurtocht naar de Betekenis van het

Begrip 'Woonmilieu'

Jaap Ketelaar

nr 28

Urban Environment in Developing Countries

editors: Peter A. Erkelens

George G. van der Meulen (red.)

nr 29

Stategische Plannen voor de Stad:

Onderzoek en Planning in Drie Steden

prof.dr. H. Fassbinder (red.)

H. Rikhof (red.)

nr 30

Stedebouwkunde en Stadsbestuur

Piet Beekman

nr 31

De Architectuur van Djenné:

Een Onderzoek naar de Historische Stad

P.C.M. Maas

nr 32

Conjoint Experiments and Retail Planning

Harmen Oppewal

nr 33

Strukturformen Indonesischer Bautechnik:

Entwicklung Methodischer Grundlagen

für eine 'Konstruktive Pattern Language'

in Indonesien

Heinz Frick arch. SIA

nr 34

Styles of Architectural Designing:

Empirical Research on Working Styles

and Personality Dispositions

Anton P.M. van Bakel

nr 35

Conjoint Choice Models for Urban

Tourism Planning and Marketing

Benedict Dellaert

nr 36

Stedelijke Planvorming als Co-Produktie Helga Fassbinder (red.) 


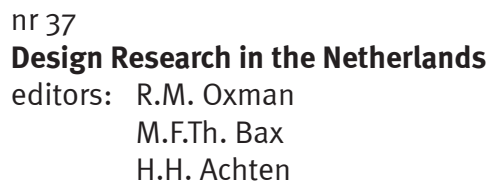

nr 38

Communication in the Building Industry

Bauke de Vries

nr 39

Optimaal Dimensioneren van

Gelaste Plaatliggers

J.B.W. Stark

F. van Pelt

L.F.M. van Gorp

B.W.E.M. van Hove

$\mathrm{nr} 40$

Huisvesting en Overwinning van Armoede

P.H. Thung

P. Beekman (red.)

nr 41

Urban Habitat:

The Environment of Tomorrow

George G. van der Meulen

Peter A. Erkelens

nr 42

A Typology of Joints

John C.M. Olie

nr 43

Modeling Constraints-Based Choices for Leisure Mobility Planning

Marcus P. Stemerding

nr 44

Activity-Based Travel Demand Modeling

Dick Ettema

nr 45

Wind-Induced Pressure Fluctuations

on Building Facades

Chris Geurts

nr 46

Generic Representations

Henri Achten

nr 47

Johann Santini Aichel:

Architectuur en Ambiguiteit

Dirk De Meyer nr 48

Concrete Behaviour in Multiaxial

Compression

Erik van Geel

nr 49

Modelling Site Selection

Frank Witlox

nr 50

Ecolemma Model

Ferdinand Beetstra

nr 51

Conjoint Approaches to Developing

Activity-Based Models

Donggen Wang

nr 52

On the Effectiveness of Ventilation

Ad Roos

nr 53

Conjoint Modeling Approaches for

Residential Group preferences

Eric Molin

nr 54

Modelling Architectural Design

Information by Features

Jos van Leeuwen

nr 55

A Spatial Decision Support System for the Planning of Retail and Service Facilities Theo Arentze

nr 56

Integrated Lighting System Assistant

Ellie de Groot

nr 57

Ontwerpend Leren, Leren Ontwerpen

J.T. Boekholt

nr 58

Temporal Aspects of Theme Park Choice

Behavior

Astrid Kemperman

nr 59

Ontwerp van een Geïndustrialiseerde

Funderingswijze

Faas Moonen 
nr 60

Merlin: A Decision Support System for Outdoor Leisure Planning

Manon van Middelkoop

nr 61

The Aura of Modernity

Jos Bosman

nr 62

Urban Form and Activity-Travel Patterns

Daniëlle Snellen

nr 63

Design Research in the Netherlands 2000 Henri Achten

nr 64

Computer Aided Dimensional Control in Building Construction

Rui Wu

nr 65

Beyond Sustainable Building

editors: Peter A. Erkelens

Sander de Jonge

August A.M. van Vliet

co-editor: Ruth J.G. Verhagen

nr 66

Das Globalrecyclingfähige Haus

Hans Löfflad

nr 67

Cool Schools for Hot Suburbs

René J. Dierkx

nr 68

A Bamboo Building Design Decision

Support Tool

Fitri Mardjono

nr 69

Driving Rain on Building Envelopes

Fabien van Mook

nr 70

Heating Monumental Churches

Henk Schellen

$\mathrm{nr} 71$

Van Woningverhuurder naar

Aanbieder van Woongenot

Patrick Dogge $\mathrm{nr} 72$

Moisture Transfer Properties of Coated Gypsum

Emile Goossens

nr 73

Plybamboo Wall-Panels for Housing

Guillermo E. González-Beltrán

nr 74

The Future Site-Proceedings

Ger Maas

Frans van Gassel

nr 75

Radon transport in

Autoclaved Aerated Concrete

Michel van der Pal

nr 76

The Reliability and Validity of Interactive Virtual Reality Computer Experiments

Amy Tan

nr 77

Measuring Housing Preferences Using

Virtual Reality and Belief Networks

Maciej A. Orzechowski

nr 78

Computational Representations of Words and Associations in Architectural Design Nicole Segers

nr 79

Measuring and Predicting Adaptation in Multidimensional Activity-Travel Patterns Chang-Hyeon Joh

nr 80

Strategic Briefing

Fayez Al Hassan

nr 81

Well Being in Hospitals

Simona Di Cicco

nr 82

Solares Bauen:

Implementierungs- und Umsetzungs-

Aspekte in der Hochschulausbildung

in Österreich

Gerhard Schuster 
nr 83

Supporting Strategic Design of Workplace Environments with Case-Based Reasoning

Shauna Mallory-Hill

nr 84

ACCEL: A Tool for Supporting Concept Generation in the Early Design Phase

Maxim Ivashkov

nr 85

Brick-Mortar Interaction in Masonry under Compression

Ad Vermeltfoort

nr 86

Zelfredzaam Wonen

Guus van Vliet

nr 87

Een Ensemble met Grootstedelijke Allure Jos Bosman

Hans Schippers

nr 88

On the Computation of Well-Structured Graphic Representations in Architectural Design

Henri Achten

nr 89

De Evolutie van een West-Afrikaanse

Vernaculaire Architectuur

Wolf Schijns

nr 90

ROMBO Tactiek

Christoph Maria Ravesloot

nr 91

External Coupling between Building Energy Simulation and Computational Fluid Dynamics

Ery Djunaedy

nr 92

Design Research in the Netherlands 2005

editors: Henri Achten

Kees Dorst

Pieter Jan Stappers

Bauke de Vries

nr 93

Ein Modell zur Baulichen Transformation

Jalil H. Saber Zaimian nr 94

Human Lighting Demands:

Healthy Lighting in an Office Environment Myriam Aries

nr 95

A Spatial Decision Support System for the Provision and Monitoring of Urban Greenspace

Claudia Pelizaro

nr 96

Leren Creëren

Adri Proveniers

nr 97

Simlandscape

Rob de Waard

nr 98

Design Team Communication

Ad den Otter

nr 99

Humaan-Ecologisch

Georiënteerde Woningbouw

Juri Czabanowski

nr 100

Hambase

Martin de Wit

nr 101

Sound Transmission through Pipe Systems and into Building Structures Susanne Bron-van der Jagt

nr 102

Het Bouwkundig Contrapunt Jan Francis Boelen

nr 103

A Framework for a Multi-Agent

Planning Support System

Dick Saarloos

nr 104

Bracing Steel Frames with Calcium

Silicate Element Walls

Bright Mweene Ng'andu

nr 105

Naar een Nieuwe Houtskeletbouw

F.N.G. De Medts 
nr 106 and 107

Niet gepubliceerd

nr 108

Geborgenheid

T.E.L. van Pinxteren

nr 109

Modelling Strategic Behaviour in Anticipation of Congestion

Qi Han

nr 110

Reflecties op het Woondomein

Fred Sanders

nr 111

On Assessment of Wind Comfort

by Sand Erosion

Gábor Dezsö

nr 112

Bench Heating in Monumental Churches

Dionne Limpens-Neilen

nr 113

RE. Architecture

Ana Pereira Roders

nr 114

Toward Applicable Green Architecture Usama El Fiky

nr 115

Knowledge Representation under Inherent Uncertainty in a Multi-Agent System for Land Use Planning Liying Ma

nr 116

Integrated Heat Air and Moisture

Modeling and Simulation

Jos van Schijndel

nr 117

Concrete Behaviour in Multiaxial

Compression

J.P.W. Bongers

nr 118

The Image of the Urban Landscape

Ana Moya Pellitero

nr 119

The Self-Organizing City in Vietnam

Stephanie Geertman nr 120

A Multi-Agent Planning Support System for Assessing Externalities of Urban Form Scenarios

Rachel Katoshevski-Cavari

nr 121

Den Schulbau Neu Denken,

Fühlen und Wollen

Urs Christian Maurer-Dietrich

nr 122

Peter Eisenman Theories and

Practices

Bernhard Kormoss

nr 123

User Simulation of Space Utilisation

Vincent Tabak

nr 125

In Search of a Complex System Model

Oswald Devisch

nr 126

Lighting at Work:

Environmental Study of Direct Effects

of Lighting Level and Spectrum on

Psycho-Physiological Variables

Grazyna Górnicka

nr 127

Flanking Sound Transmission through Lightweight Framed Double Leaf Walls

Stefan Schoenwald

nr 128

Bounded Rationality and Spatio-Temporal Pedestrian Shopping Behavior

Wei Zhu

nr 129

Travel Information:

Impact on Activity Travel Pattern

Zhongwei Sun

nr 130

Co-Simulation for Performance

Prediction of Innovative Integrated

Mechanical Energy Systems in Buildings

Marija Trčka

nr 131

Niet gepubliceerd 
nr 132

Architectural Cue Model in Evacuation Simulation for Underground Space Design Chengyu Sun

nr 133

Uncertainty and Sensitivity Analysis in Building Performance Simulation for Decision Support and Design Optimization Christina Hopfe

nr 134

Facilitating Distributed Collaboration in the AEC/FM Sector Using Semantic Web Technologies

Jacob Beetz

nr 135

Circumferentially Adhesive Bonded Glass Panes for Bracing Steel Frame in Façades

Edwin Huveners

nr 136

Influence of Temperature on Concrete

Beams Strengthened in Flexure with CFRP

Ernst-Lucas Klamer

nr 137

Sturen op Klantwaarde

Jos Smeets

nr 139

Lateral Behavior of Steel Frames

with Discretely Connected Precast Concrete Infill Panels

Paul Teewen

nr 140

Integral Design Method in the Context

of Sustainable Building Design

Perica Savanović

nr 141

Household Activity-Travel Behavior:

Implementation of Within-Household

Interactions

Renni Anggraini

$\mathrm{nr} 142$

Design Research in the Netherlands 2010

Henri Achten nr 143

Modelling Life Trajectories and Transport Mode Choice Using Bayesian Belief Networks Marloes Verhoeven

nr 144

Assessing Construction Project Performance in Ghana

William Gyadu-Asiedu

nr 145

Empowering Seniors through

Domotic Homes

Masi Mohammadi

nr 146

An Integral Design Concept for Ecological Self-Compacting Concrete

Martin Hunger

nr 147

Governing Multi-Actor Decision Processes in Dutch Industrial Area Redevelopment Erik Blokhuis

nr 148

A Multifunctional Design Approach for Sustainable Concrete

Götz Hüsken

nr 149

Quality Monitoring in Infrastructural Design-Build Projects

Ruben Favié

nr 150

Assessment Matrix for Conservation of Valuable Timber Structures

Michael Abels

nr 151

Co-simulation of Building Energy Simulation and Computational Fluid Dynamics for Whole-Building Heat, Air and Moisture Engineering Mohammad Mirsadeghi

nr 152

External Coupling of Building Energy Simulation and Building Element Heat, Air and Moisture Simulation Daniel Cóstola 
nr 153

Adaptive Decision Making In

Multi-Stakeholder Retail Planning

Ingrid Janssen

nr 154

Landscape Generator

Kymo Slager

nr 155

Constraint Specification in Architecture

Remco Niemeijer

nr 156

A Need-Based Approach to Dynamic Activity Generation

Linda Nijland

nr 157

Modeling Office Firm Dynamics in an Agent-Based Micro Simulation Framework Gustavo Garcia Manzato

nr 158

Lightweight Floor System for

Vibration Comfort

Sander Zegers

nr 159

Aanpasbaarheid van de Draagstructuur Roel Gijsbers

nr 160

'Village in the City' in Guangzhou, China Yanliu Lin

nr 161

Climate Risk Assessment in Museums

Marco Martens

nr 162

Social Activity-Travel Patterns

Pauline van den Berg

nr 163

Sound Concentration Caused by

Curved Surfaces

Martijn Vercammen

nr 164

Design of Environmentally Friendly

Calcium Sulfate-Based Building Materials:

Towards an Improved Indoor Air Quality

Qingliang Yu nr 165

Beyond Uniform Thermal Comfort on the Effects of Non-Uniformity and Individual Physiology

Lisje Schellen

nr 166

Sustainable Residential Districts

Gaby Abdalla

nr 167

Towards a Performance Assessment Methodology using Computational Simulation for Air Distribution System Designs in Operating Rooms

Mônica do Amaral Melhado

nr 168

Strategic Decision Modeling in Brownfield Redevelopment

Brano Glumac

nr 169

Pamela: A Parking Analysis Model for Predicting Effects in Local Areas Peter van der Waerden

nr 170

A Vision Driven Wayfinding Simulation-System Based on the Architectural Features Perceived in the Office Environment

Qunli Chen

nr 171

Measuring Mental Representations Underlying Activity-Travel Choices

Oliver Horeni

nr 172

Modelling the Effects of Social Networks on Activity and Travel Behaviour Nicole Ronald

nr 173

Uncertainty Propagation and Sensitivity Analysis Techniques in Building Performance Simulation to Support Conceptual Building and System Design

Christian Struck

nr 174

Numerical Modeling of Micro-Scale Wind-Induced Pollutant Dispersion in the Built Environment

Pierre Gousseau 
nr 175

Modeling Recreation Choices

over the Family Lifecycle

Anna Beatriz Grigolon

nr 176

Experimental and Numerical Analysis of Mixing Ventilation at Laminar, Transitional and Turbulent Slot Reynolds Numbers

Twan van Hooff

nr 177

Collaborative Design Support:

Workshops to Stimulate Interaction and Knowledge Exchange Between Practitioners

Emile M.C.J. Quanjel

nr 178

Future-Proof Platforms for Aging-in-Place

Michiel Brink

nr 179

Motivate:

A Context-Aware Mobile Application for Physical Activity Promotion

Yuzhong Lin

nr 180

Experience the City:

Analysis of Space-Time Behaviour and

Spatial Learning

Anastasia Moiseeva

nr 181

Unbonded Post-Tensioned Shear Walls of Calcium Silicate Element Masonry

Lex van der Meer

nr 182

Construction and Demolition Waste

Recycling into Innovative Building Materials for Sustainable Construction in Tanzania

Mwita M. Sabai

nr 183

Durability of Concrete

with Emphasis on Chloride Migration

Przemystaw Spiesz

nr 184

Computational Modeling of Urban

Wind Flow and Natural Ventilation Potential

of Buildings

Rubina Ramponi nr 185

A Distributed Dynamic Simulation Mechanism for Buildings Automation and Control Systems

Azzedine Yahiaoui

nr 186

Modeling Cognitive Learning of Urban Networks in Daily Activity-Travel Behavior Şehnaz Cenani Durmazoğlu

nr 187

Functionality and Adaptability of Design Solutions for Public Apartment Buildings in Ghana

Stephen Agyefi-Mensah

nr 188

A Construction Waste Generation Model for Developing Countries

Lilliana Abarca-Guerrero

nr 189

Synchronizing Networks:

The Modeling of Supernetworks for Activity-Travel Behavior

Feixiong Liao

nr 190

Time and Money Allocation Decisions in Out-of-Home Leisure Activity Choices Gamze Zeynep Dane

nr 191

How to Measure Added Value of CRE and Building Design

Rianne Appel-Meulenbroek

nr 192

Secondary Materials in Cement-Based

Products:

Treatment, Modeling and Environmental Interaction

Miruna Florea

nr 193

Concepts for the Robustness Improvement of Self-Compacting Concrete:

Effects of Admixtures and Mixture Components on the Rheology and Early Hydration at Varying Temperatures Wolfram Schmidt 
nr 194

Modelling and Simulation of Virtual Natural Lighting Solutions in Buildings

Rizki A. Mangkuto

nr 195

Nano-Silica Production at Low Temperatures from the Dissolution of Olivine - Synthesis, Tailoring and Modelling

Alberto Lazaro Garcia

nr 196

Building Energy Simulation Based

Assessment of Industrial Halls for

Design Support

Bruno Lee

nr 197

Computational Performance Prediction

of the Potential of Hybrid Adaptable

Thermal Storage Concepts for Lightweight

Low-Energy Houses

Pieter-Jan Hoes

nr 198

Application of Nano-Silica in Concrete

George Quercia Bianchi

nr 199

Dynamics of Social Networks and Activity

Travel Behaviour

Fariya Sharmeen

nr 200

Building Structural Design Generation and Optimisation including Spatial Modification Juan Manuel Davila Delgado

nr 201

Hydration and Thermal Decomposition of Cement/Calcium-Sulphate Based Materials Ariën de Korte

nr 202

Republiek van Beelden:

De Politieke Werkingen van het Ontwerp in Regionale Planvorming

Bart de Zwart

nr 203

Effects of Energy Price Increases on Individual Activity-Travel Repertoires and Energy Consumption

Dujuan Yang nr 204

Geometry and Ventilation:

Evaluation of the Leeward Sawtooth Roof

Potential in the Natural Ventilation of

Buildings

Jorge Isaac Perén Montero

nr 205

Computational Modelling of Evaporative Cooling as a Climate Change Adaptation Measure at the Spatial Scale of Buildings and Streets

Hamid Montazeri

nr 206

Local Buckling of Aluminium Beams in Fire Conditions

Ronald van der Meulen

nr 207

Historic Urban Landscapes:

Framing the Integration of Urban and Heritage Planning in Multilevel Governance Loes Veldpaus

nr 208

Sustainable Transformation of the Cities: Urban Design Pragmatics to Achieve a Sustainable City

Ernesto Antonio Zumelzu Scheel

nr 209

Development of Sustainable Protective Ultra-High Performance Fibre Reinforced Concrete (UHPFRC):

Design, Assessment and Modeling

Rui Yu

nr 210

Uncertainty in Modeling Activity-Travel Demand in Complex Uban Systems Soora Rasouli

nr 211

Simulation-based Performance Assessment of Climate Adaptive Greenhouse Shells Chul-sung Lee

nr 212

Green Cities:

Modelling the Spatial Transformation of the Urban Environment using Renewable Energy Technologies

Saleh Mohammadi 
nr 213

A Bounded Rationality Model of Short and Long-Term Dynamics of Activity-Travel

Behavior

Ifigeneia Psarra

nr 214

Effects of Pricing Strategies on Dynamic Repertoires of Activity-Travel Behaviour Elaheh Khademi

nr 215

Handstorm Principles for Creative and Collaborative Working

Frans van Gassel

nr 216

Light Conditions in Nursing Homes:

Visual Comfort and Visual Functioning of

Residents

Marianne M. Sinoo

nr 217

Woonsporen:

De Sociale en Ruimtelijke Biografie van een Stedelijk Bouwblok in de Amsterdamse Transvaalbuurt

Hüseyin Hüsnü Yegenoglu

nr 218

Studies on User Control in Ambient

Intelligent Systems

Berent Willem Meerbeek

nr 219

Daily Livings in a Smart Home:

Users' Living Preference Modeling of Smart Homes

Erfaneh Allameh

nr 220

Smart Home Design:

Spatial Preference Modeling of Smart

Homes

Mohammadali Heidari Jozam

nr 221

Wonen:

Discoursen, Praktijken, Perspectieven

Jos Smeets

nr 222

Personal Control over Indoor Climate in

Offices:

Impact on Comfort, Health and Productivity

Atze Christiaan Boerstra nr 223

Personalized Route Finding in Multimodal Transportation Networks

Jianwe Zhang

nr 224

The Design of an Adaptive Healing Room for Stroke Patients

Elke Daemen

nr 225

Experimental and Numerical Analysis of Climate Change Induced Risks to Historic Buildings and Collections

Zara Huijbregts

nr 226

Wind Flow Modeling in Urban Areas Through Experimental and Numerical Techniques Alessio Ricci

nr 227

Clever Climate Control for Culture:

Energy Efficient Indoor Climate Control

Strategies for Museums Respecting

Collection Preservation and Thermal

Comfort of Visitors

Rick Kramer

nr 228

Fatigue Life Estimation of Metal Structures Based on Damage Modeling

Sarmediran Silitonga

nr 229

A multi-agents and occupancy based strategy for energy management and process control on the room-level Timilehin Moses Labeodan

nr 230

Environmental assessment of Building Integrated Photovoltaics:

Numerical and Experimental Carrying Capacity Based Approach Michiel Ritzen

nr 231

Performance of Admixture and Secondary Minerals in Alkali Activated Concrete: Sustaining a Concrete Future Arno Keulen 
nr 232

World Heritage Cities and Sustainable Urban Development:

Bridging Global and Local Levels in Monitoring the Sustainable Urban Development of World Heritage Cities

Paloma C. Guzman Molina

nr 233

Stage Acoustics and Sound Exposure in Performance and Rehearsal Spaces for Orchestras:

Methods for Physical Measurements Remy Wenmaekers

nr 234

Municipal Solid Waste Incineration (MSWI)

Bottom Ash:

From Waste to Value Characterization, Treatments and Application

Pei Tang

nr 235

Large Eddy Simulations Applied to Wind Loading and Pollutant Dispersion Mattia Ricci

nr 236

Alkali Activated Slag-Fly Ash Binders:

Design, Modeling and Application

Xu Gao

nr 237

Sodium Carbonate Activated Slag:

Reaction Analysis, Microstructural Modification \& Engineering Application Bo Yuan

nr 238

Shopping Behavior in Malls

Widiyani

nr 239

Smart Grid-Building Energy Interactions:

Demand Side Power Flexibility in Office

Buildings

Kennedy Otieno Aduda

nr 240

Modeling Taxis Dynamic Behavior in Uncertain Urban Environments

Zheng Zhong nr 241

Gap-Theoretical Analyses of Residential Satisfaction and Intention to Move

Wen Jiang

nr 242

Travel Satisfaction and Subjective Well-Being: A Behavioral Modeling Perspective Yanan Gao

nr 243

Building Energy Modelling to Support the Commissioning of Holistic Data Centre Operation

Vojtech Zavrel

nr 244

Regret-Based Travel Behavior Modeling:

An Extended Framework

Sunghoon Jang

nr 245

Towards Robust Low-Energy Houses:

A Computational Approach for Performance Robustness Assessment using Scenario Analysis

Rajesh Reddy Kotireddy

nr 246

Development of sustainable and functionalized inorganic binder-biofiber composites

Guillaume Doudart de la Grée

nr 247

A Multiscale Analysis of the Urban Heat Island Effect: From City Averaged Temperatures to the Energy Demand of Individual Buildings

Yasin Toparlar

nr 248

Design Method for Adaptive Daylight Systems for buildings covered by large (span) roofs

Florian Heinzelmann

nr 249

Hardening, high-temperature resistance and acid resistance of one-part geopolymers Patrick Sturm 
nr 250

Effects of the built environment on dynamic repertoires of activity-travel behaviour

Aida Pontes de Aquino

nr 251

Modeling for auralization of urban environments: Incorporation of directivity in sound propagation and analysis of a framework for auralizing a car pass-by

Fotis Georgiou

nr 252

Wind Loads on Heliostats and Photovoltaic Trackers

Andreas Pfahl

nr 253

Approaches for computational performance optimization of innovative adaptive façade concepts

Roel Loonen

nr 254

Multi-scale FEM-DEM Model for Granular

Materials: Micro-scale boundary conditions, Statics, and Dynamics

Jiadun Liu

nr 255

Bending Moment - Shear Force Interaction of Rolled I-Shaped Steel Sections

Rianne Willie Adriana Dekker

nr 256

Paralympic tandem cycling and handcycling: Computational and wind tunnel analysis of aerodynamic performance Paul Fionn Mannion

nr 257

Experimental characterization and numerical modelling of $3 \mathrm{D}$ printed concrete: Controlling structural behaviour in the fresh and hardened state

Robert Johannes Maria Wolfs

nr 258

Requirement checking in the building industry: Enabling modularized and extensible requirement checking systems based on semantic web technologies

Chi Zhang nr 259

A Sustainable Industrial Site Redevelopment Planning Support System

Tong Wang

nr 260

Efficient storage and retrieval of detailed building models: Multi-disciplinary and long-term use of geometric and semantic construction information

Thomas Ferdinand Krijnen

nr 261

The users' value of business center concepts for knowledge sharing and networking behavior within and between organizations Minou Weijs-Perrée

nr 262

Characterization and improvement of aerodynamic performance of vertical axis wind turbines using computational fluid dynamics (CFD)

Abdolrahim Rezaeiha

nr 263

In-situ characterization of the acoustic impedance of vegetated roofs

Chang Liu

nr 264

Occupancy-based lighting control: Developing an energy saving strategy that ensures office workers' comfort

Christel de Bakker

nr 265

Stakeholders-Oriented Spatial Decision

Support System

Cahyono Susetyo

nr 266

Climate-induced damage in oak museum objects

Rianne Aleida Luimes

nr 267

Towards individual thermal comfort:

Model predictive personalized control of heating systems

Katarina Katic 
nr 268

Modelling and Measuring Quality of Urban Life: Housing, Neighborhood, Transport and Job

Lida Aminian

nr 269

Optimization of an aquifer thermal energy storage system through integrated modelling of aquifer, HVAC systems and building Basar Bozkaya

nr 270

Numerical modeling for urban sound propagation: developments in wave-based and energy-based methods

Raúl Pagán Muñoz

nr 271

Lighting in multi-user office environments: improving employee wellbeing through personal control

Sanae van der Vleuten-Chraibi

nr 272

A strategy for fit-for-purpose occupant behavior modelling in building energy and comfort performance simulation

Isabella I. Gaetani dell'Aquila d'Aragona

nr 273

Een architectuurhistorische waardestelling van naoorlogse woonwijken in Nederland: Het voorbeeld van de Westelijke Tuinsteden in Amsterdam

Eleonore Henriette Marie Mens

nr 274

Job-Housing Co-Dependent Mobility

Decisions in Life Trajectories

Jia Guo

nr 275

A user-oriented focus to create healthcare facilities: decision making on strategic values

Emilia Rosalia Catharina Maria Huisman

nr 276

Dynamics of plane impinging jets at moderate Reynolds numbers with applications to air curtains Adelya Khayrullina nr 277

Valorization of Municipal Solid Waste Incineration Bottom Ash - Chemical Nature, Leachability and Treatments of Hazardous Elements

Qadeer Alam

nr 278

Treatments and valorization of MSWI bottom ash - application in cement-based materials

Veronica Caprai

nr 279

Personal lighting conditions of office workers - input for intelligent systems to optimize subjective alertness

Juliëtte van Duijnhoven

nr 280

Social influence effects in tourism travel: air trip itinerary and destination choices

Xiaofeng Pan

nr 281

Advancing Post-War Housing: Integrating Heritage Impact, Environmental Impact, Hygrothermal Risk and Costs in Renovation Design Decisions

Lisanne Claartje Havinga

nr 282

Impact resistant ultra-high performance fibre reinforced concrete: materials, components and properties

Peipeng Li

nr 283

Demand-driven Science Parks: The Perceived Benefits and Trade-offs of Tenant Firms with regard to Science Park Attributes Wei Keat Benny Ng

nr 284

Raise the lantern; how light can help to maintain a healthy and safe hospital environment focusing on nurses Maria Petronella Johanna Aarts

nr 285

Modelling Learning and Dynamic Route and Parking Choice Behaviour under Uncertainty Elaine Cristina Schneider de Carvalho 
nr 286

Identifying indoor local microclimates for safekeeping of cultural heritage

Karin Kompatscher

nr 287

Probabilistic modeling of fatigue resistance for welded and riveted bridge details. Resistance models and estimation of uncertainty.

Davide Leonetti

nr 288

Performance of Layered UHPFRC under Static and Dynamic Loads: Effects of steel fibers, coarse aggregates and layered structures

Yangyueye Cao

nr 289

Photocatalytic abatement of the nitrogen oxide pollution: synthesis, application and long-term evaluation of titania-silica composites

Yuri Hendrix

nr 290

Assessing knowledge adoption in postdisaster reconstruction: Understanding the impact of hazard-resistant construction knowledge on reconstruction processes of self-recovering communities in Nepal and the Philippines

Eefje Hendriks

nr 291

Locating electric vehicle charging stations: A multi-agent based dynamic simulation Seheon Kim

nr 292

De invloed van Lean Management op de beheersing van het bouwproces

Wim van den Bouwhuijsen

nr 293

Neighborhood Environment and Physical Activity of Older Adults

Zhengying Liu

nr 294

Practical and continuous luminance distribution measurements for lighting quality Thijs Willem Kruisselbrink nr 295

Auditory Distraction in Open-Plan Study Environments in Higher Education

Pieternella Elizabeth Braat-Eggen

nr 296

Exploring the effect of the sound environment on nurses' task performance: an applied approach focusing on prospective memory

Jikke Reinten

nr 297

Design and performance of water resistant cementitious materials- Mechanisms, evaluation and applications

Zhengyao Qu

nr 298

Design Optimization of Seasonal Thermal Energy Storage Integrated District Heating and Cooling System: A Modeling and Simulation Approach

Luyi Xu

nr 299

Land use and transport: Integrated approaches for planning and management Zhongqi Wang

nr 300

Multi-disciplinary optimization of building spatial designs: co-evolutionary design process simulations, evolutionary algorithms, hybrid approaches Sjonnie Boonstra

nr 301

Modeling the spatial and temporal relation between urban land use, temperature, and energy demand Hung-Chu Chen

nr 302

Seismic retrofitting of masonry walls with flexible deep mounted CFRP strips Ömer Serhat Türkmen 